Florida International University FIU Digital Commons

3-30-2017

\title{
Evaluation and Predictability of Observation-based Surface Wind Asymmetric Structure in Tropical Cyclones
}

Bradley Klotz

bklot003@fiu.edu

DOI: $10.25148 /$ etd.FIDC001738

Follow this and additional works at: https://digitalcommons.fiu.edu/etd

Part of the Atmospheric Sciences Commons, and the Meteorology Commons

\section{Recommended Citation}

Klotz, Bradley, "Evaluation and Predictability of Observation-based Surface Wind Asymmetric Structure in Tropical Cyclones" (2017). FIU Electronic Theses and Dissertations. 3207.

https://digitalcommons.fiu.edu/etd/3207 


\title{
FLORIDA INTERNATIONAL UNIVERSITY \\ Miami, Florida
}

\section{EVALUATION AND PREDICTABILITY OF OBSERVATION-BASED SURFACE WIND ASYMMETRIC STRUCTURE IN TROPICAL CYCLONES}

\author{
A dissertation submitted in partial fulfillment of \\ the requirements for the degree of \\ DOCTOR OF PHILOSOPHY \\ in \\ GEOSCIENCES \\ by \\ Bradley Klotz
}


To: Dean Michael R. Heithaus

College of Arts, Sciences and Education

This dissertation, written by Bradley Klotz, and entitled Evaluation and Predictability of Observation-based Surface Wind Asymmetric Structure in Tropical Cyclones, having been approved in respect to style and intellectual content, is referred to you for judgment.

We have read this dissertation and recommend that it be approved.

Hugh Willoughby

Ping Zhu

Robert Burgman

Wei Wang

Haiyan Jiang, Major Professor

Date of Defense: March 30, 2017

The dissertation of Bradley Klotz is approved.

Dean Michael R. Heithaus College of Arts, Sciences and Education

Andrés G. Gil

Vice President for Research and Economic Development and Dean of the University Graduate School

Florida International University, 2017 


\section{DEDICATION}

I would like to dedicate this dissertation to the memory of my grandmothers,

Dorothy Klotz and Katheryne Wilson. They were great sources of support and encouraged me to pursue my dreams at a young age. 


\section{ACKNOWLEDGMENTS}

I would first like to thank my advisor Dr. Haiyan Jiang for her assistance and guidance in accomplishing this work. Many discussions were held to obtain the best results possible, and her suggestions were vital to my thought and work process throughout the study. I also would like to give my gratitude to my committee members: Drs. Hugh Willoughby, Robert Burgman, Ping Zhu, and Wei Wang. Their comments and suggestions sparked ideas that improved the quality of my work. My appreciation also goes to my fellow graduate students and group members including Yongxian Pei, Cheng Tao, Jonathan Zawislak, Margie Kieper, and Tyler Wieland. Our weekly meetings brought about interesting discussions that were helpful for improving my understanding of topics related to this work.

Special appreciation is given to several of my coworkers (current and former) at NOAA's Hurricane Research Division for their help, support, and understanding during my time as a graduate student at FIU. I would especially like to thank Drs. Eric Uhlhorn and Frank Marks for encouraging me to pursue my degree. Additionally, the comments provided by Dr. Paul Reasor, Dr. Jun Zhang, and Bachir Annane significantly improved the results provided in Chapters 2-4.

I would also like to thank my family for their love and support from start to finish. I especially want to thank my wife Kristie for allowing me to spend these last three and a half years to work toward my degree. She loved and encouraged me when I needed it most and kept me moving forward. I would also like to thank my children Sam and Tori for their patience during this time and for giving me motivation to keep working hard. Additionally, I'd like to thank my parents, Wayne and Karen Klotz for their 
encouragement over the years and for helping financially support my schooling over the last year and a half. James and Angie Pramann, my parents-in-law, were also very supportive of me during my studies and always expressed interest in my work over the years I was at FIU. Last but certainly not least, I want to thank God for opening this door for me to pursue my goal. He kept me focused and reminded me that while this is a difficult task, he was with me every step of the way. I would have nothing without him and the saving grace he provides through His Son Jesus. He deserves all the praise for letting me complete this degree.

Financial support for this study was partially provided through my advisor by the NASA New Investigator Program (NIP) Award NNX10AG55G and NASA Hurricane Science Research Program (HSRP) Grant NNX10AG34G under the direction of Ramesh Kakar. 


\title{
ABSTRACT OF THE DISSERTATION \\ EVALUATION AND PREDICTABILITY OF OBSERVATION-BASED SURFACE WIND ASYMMETRIC STRUCTURE IN TROPICAL CYCLONES
}

\author{
by \\ Bradley Klotz \\ Florida International University, 2017 \\ Miami, Florida \\ Professor Haiyan Jiang, Major Professor
}

Surface wind speeds are an important and revealing component of the structure of tropical cyclones (TCs). To understand the asymmetric structure of surface winds in TCs associated with differences in formation region, environmental wind shear, storm forward motion, and TC strength and intensification, a twelve year database of satellite scatterometer data are utilized to produce composite total wind speed and Fourierderived, low wavenumber analyses. A quantified asymmetry is determined as a function of TC intensity and reveals the tropical storms are influenced by wind shear at all TCcentric radii but only for areas away from the radius of maximum wind in hurricanes. Additionally, an increase of absolute angular momentum flux has a preference for the downshear-right quadrant, and the low wavenumber maximum develops downwind of this momentum transport. Further evaluation of the asymmetric structure with respect to wind shear's relation to motion and impacts during $\mathrm{TC}$ intensity change are also considered.

A composite rapid intensification event is produced and compared to overlapping satellite rain estimates. Results indicate that the TC becomes more symmetric during 
intensification and the phase of the maximum asymmetry rotates from a downshear-left direction to upshear-left direction after the intensification slows. The rain or convective maximum is generally located upwind of the surface wind maximum at the early stages of intensification and is coincident with the region of large angular momentum transport, which supports the idea that the surface wind asymmetry is likely a consequence of convective or other processes. Using data from a regional TC model, it is also determined that the scatterometer data are useful for model verification of tropical storms and nonmajor hurricanes and performs similar to or better than the standard tool at forecast lead times up to 60 hours. Preliminary comparisons of model-derived surface wind asymmetry relative to rain generally confirm the observational results. 


\section{TABLE OF CONTENTS}

CHAPTER

PAGE

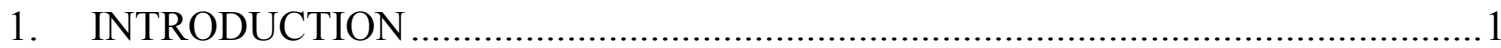

1.1. Background and Motivation of Work.............................................................

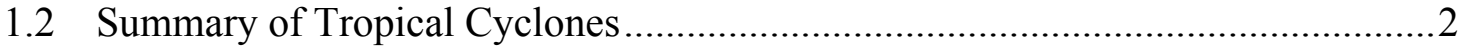

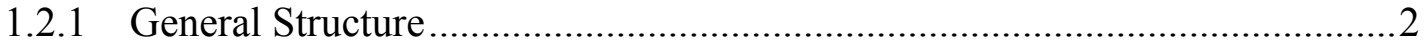

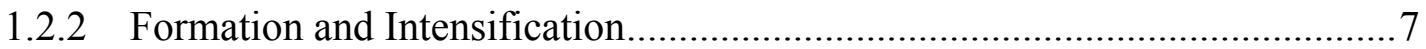

1.3 Observing Tropical Cyclones and Their Surface Winds ...................................... 10

1.3.1 Aircraft Reconnaissance ............................................................................. 11

1.3.2 Observations from Satellite-based Instruments: Scatterometry ………….....13

1.4 The State of Tropical Cyclone Forecasting …………….....................................15

1.5 Expectations and Considerations......................................................................... 17

2. GLOBAL COMPOSITES OF SURFACE WIND SPEEDS IN TROPICAL CYCLONES BASED ON A 12-YEAR SCATTEROMETER DATABASE …………....19

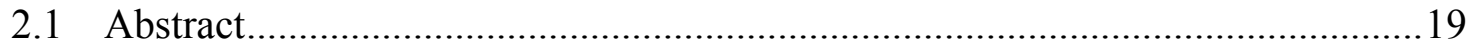

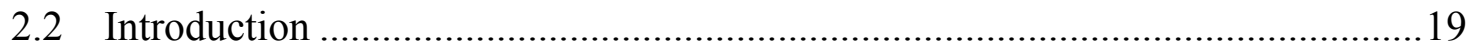

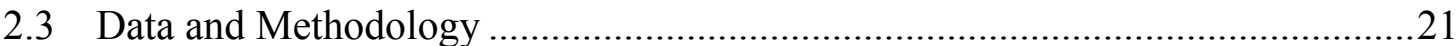

2.3.1 Determination of a Radius of Maximum Wind from Scatterometer Data ....24

2.3.2 Scatterometer Verification and Other Data Considerations ...........................27

2.4 Comparison to Theoretical and Observational Studies Using Basin-

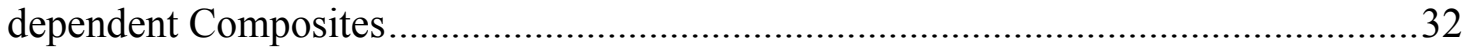

2.5 Wind Shear and Storm Motion Impact based on TC Intensity .............................36

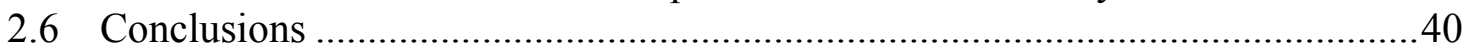

3. EXAMINATION OF SURFACE WIND ASYMMETRIES IN TROPICAL CYCLONES: PART I. GENERAL STRUCTURE AND WIND SHEAR IMPACTS ....42

3.1 Abstract..........................................................................................4

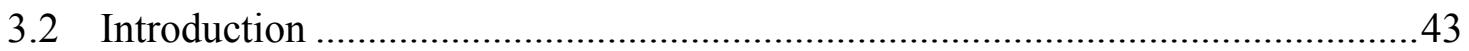

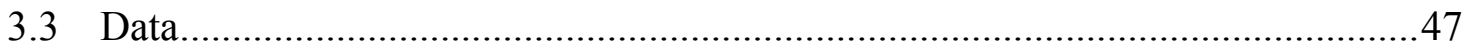

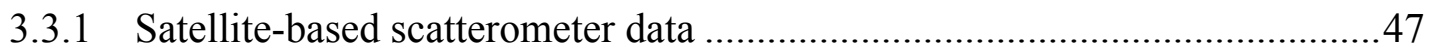

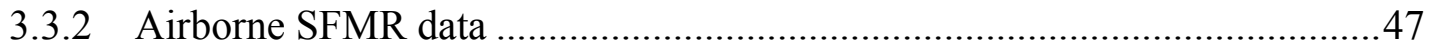

3.3.3 TC Intensity, Motion, and Vertical Wind Shear Data ....................................48

3.4 Methods for Producing Low Wavenumber Analyses with Scatterometer Data...49

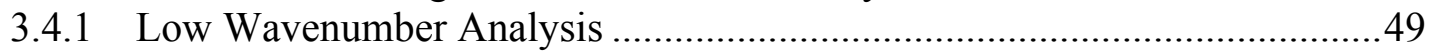

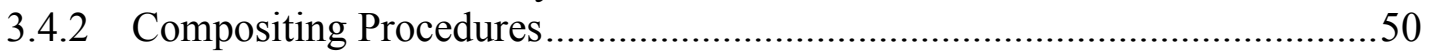

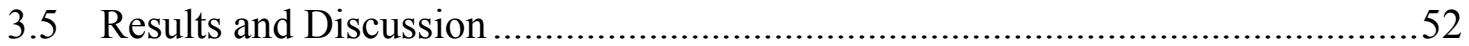

3.5.1 Validation of the Scatterometer Analysis Method Using SFMR Data..........52

3.5.2 Asymmetric Structure as a Function of TC Intensity ......................................54

3.5.3 Relative Contribution of Wind Shear on TC Surface Wind Structure ...........57

3.5.4 Angular Difference Between Shear and Motion and Its Influence on

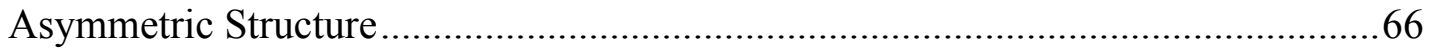

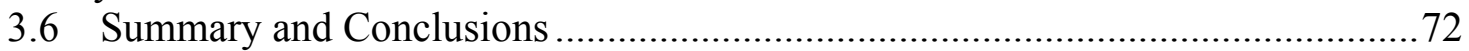




\section{EXAMINATION OF SURFACE WIND ASYMMETRIES IN TROPICAL}

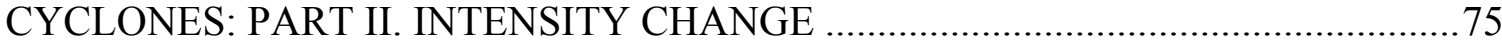

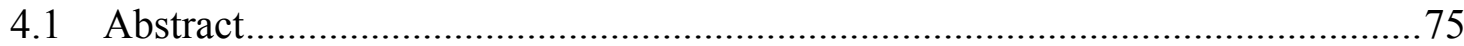

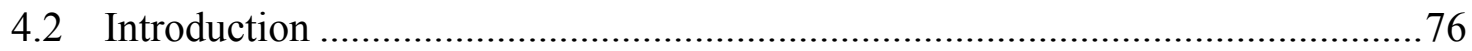

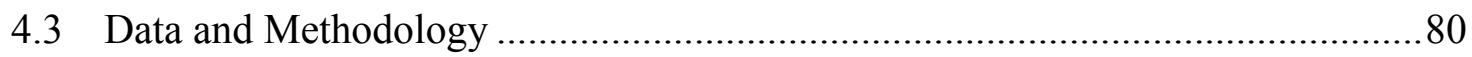

4.3.1 Summary of Scatterometer Data and Intensity Metrics .................................81

4.3.2 TRMM Microwave Imager (TMI) Data …………....................................... 81

4.3.3 Methods Specific to Intensity Change Analysis............................................82

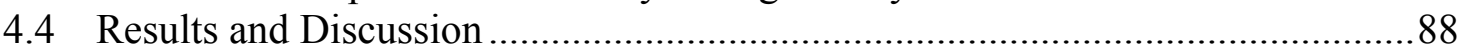

4.4.1 Intensity Change Dependence on Motion and Shear ....................................8 88

4.4.2 Composite Analysis of Intensity Change Groups ……………………….......8

4.4.3 An RI Event as Observed by QuikSCAT: Typhoon Parma ………………....96

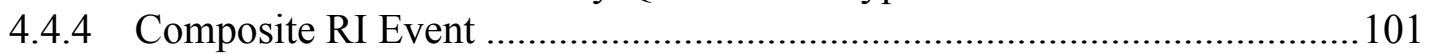

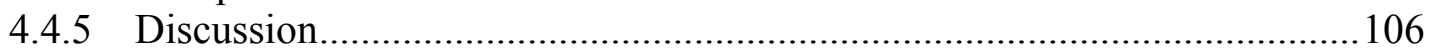

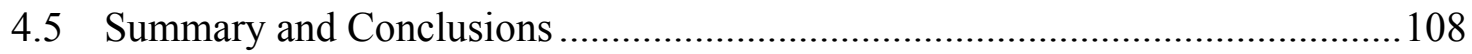

\section{PREDICTABILITY AND VERIFICATION OF SURFACE WIND} STRUCTURE IN TROPICAL CYCLONES .........................................................111

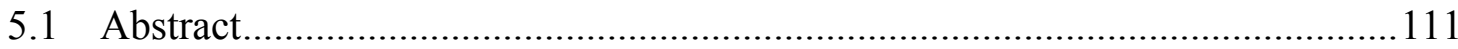

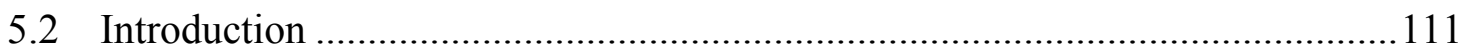

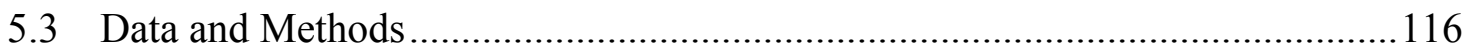

5.3.1 Description of Best Track and Dvorak Data and Their Use........................116

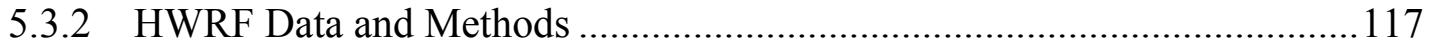

5.4 Scatterometer TC Intensity Metric Compared to Similar Best Track, Dvorak,

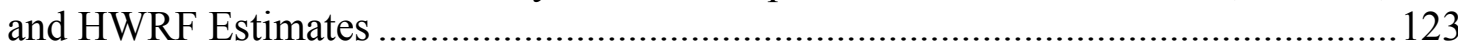

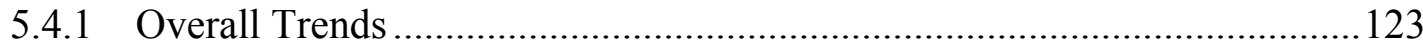

5.4.2 Tropical Cyclone Basin Dependency ……………….................................. 126

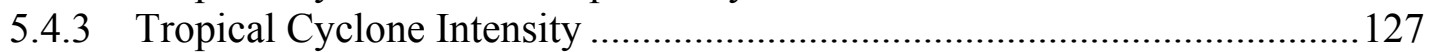

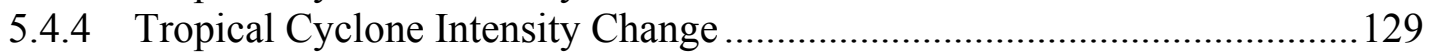

5.5 Effectiveness of Using Scatterometer Data for Tropical Cyclone Intensity

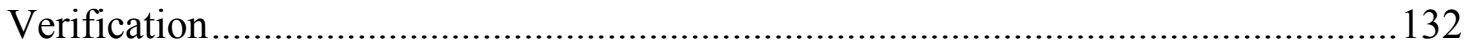

5.6 Preliminary Analysis of Rain-Relative Wind Structure .................................... 137

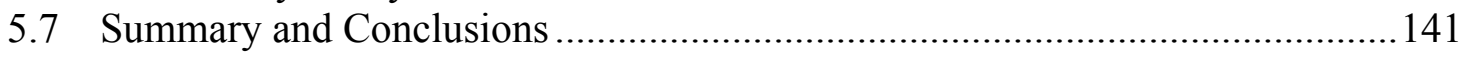

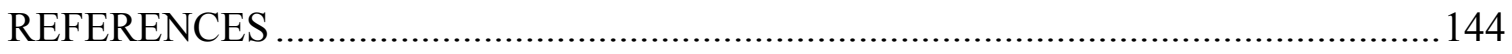

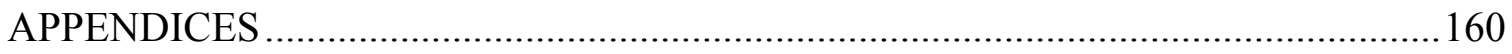

VITA 


\section{LIST OF TABLES}

TABLE

PAGE

Table 1.1. Included are the Saffir-Simpson hurricane wind classifications (modified from Simpson and Riehl 1981). Note that wind speeds are in miles per hour (mph), where $1 \mathrm{mph}=0.869 \mathrm{kt}$ or $0.447 \mathrm{~m} \mathrm{~s}^{-1}$.

Table 2.1. Basic statistics including mean, median, minimum, maximum, and peak percentage of RMW for each examined TC basin. RMW and frequency of occurrence are listed for the peak value. Similar values are provided for RMW determined from SFMR flights over the North Atlantic (U14).

Table 3.1. A compiled list of coincident SFMR and scatterometer cases is provided and separated based on their best-track intensity. In parentheses is the SFMR offset in hours relative to the scatterometer time.

Table 3.2. Median values for motion and wind shear speed, direction, and direction difference are provided for each $\Delta \theta_{\mathrm{sm}}$ group. Error estimates indicate $95 \%$ confidence intervals. .

Table 4.1. The number of cases for each intensity change classification (RI, SI, SS, and $\mathrm{WK}$ ) is provided as a function of TC basin. Additional indicators for RII and RIC cases as well as for intensifying and non-intensifying (INTS vs. NINTS) cases are provided too. Values in parentheses represent the percentage first within the respective intensity change group followed by the percentage within the basin. For the INTS and NINTS labels, values in parentheses indicate the number and percentage of the total sample.

Table 4.2. The number of cases associated with each RI event period is provided along with the percent contribution to the sample size. Nomenclature in the period labels are $t$ for the respective time, $\mathrm{RI}_{0}$ for the time marking the start of $\mathrm{RI}$, and $\mathrm{RI}_{\mathrm{E}}$ for the time marking the end of the RI event......

Table 4.3. Provided are median and standard deviation values of various environmental and storm parameters within the RII and RIC groups. Note that $\alpha_{1, \max }$ is relative to the shear vector with storm motion removed.

Table 5.1. Provided is a list of the different physical schemes and parameterizations used in the 2015 operational version of HWRF. Entries marked with an asterisk are new to this version. A list of related references is provided for each scheme or parameterization.

Table 5.2. Listed are the model cases and number of runs available from the HWRF dataset for North Atlantic and Eastern Pacific TCs. Also included is the number of cases with at least one overlapping scatterometer time. 
Table 5.3. Provided are the $\varepsilon$ values taken from their CDFs and represent the lowest $10 \%$ (first sub-column), interquartile range (25-75\%, second sub-column), and highest $90 \%$ (third sub-column) for the model, SFMR, and scatterometer datasets. These values are stratified according to the defined TC intensity and intensity change groups. 


\section{LIST OF FIGURES}

FIGURE

PAGE

Figure 1.1. Taken from Smith (2006), this schematic diagram shows the ideal primary TC circulation in gradient balance with respective forces indicated with the text.

Figure 1.2. The schematic diagram of the secondary circulation of a mature tropical cyclone is displayed [taken from Willoughby (1988)]. Black arrows indicate the air flow direction

Figure 1.3. From Hawkins and Rubsam (1968), the vertical temperature anomaly from Hurricane Hilda (1964) is shown. Anomalies are plotted in increments of $1{ }^{\circ} \mathrm{C} . \ldots . .5$

Figure 1.4. A schematic diagram derived from airborne radar depicts the main characteristics of the hurricane convection and precipitation [taken from Willoughby (1988)]

Figure 1.5. Adapted from Jorgensen (1984), a cross-section schematic of the expected convective structure of a mature hurricane is shown. Data for this display was gathered from radar data collected in Hurricane Allen (1980).

Figure 1.6. From Uhlhorn and Black (2003), the radiative transfer schematic diagram is provided for the airborne stepped frequency microwave radiometer (SFMR).

Figure 1.7. The scanning geometry of the QuikSCAT scatterometer is provided (from Spencer et al. 1997).

Figure 2.1. QuikSCAT scatterometer surface wind vectors are shown for Hurricane Katrina on 28 August, 2005. The left panel provides the uncorrected wind speeds and the right panel displays the neural network corrected wind speeds (kt, color scale, where $1 \mathrm{kt} \approx 0.5 \mathrm{~m} \mathrm{~s}^{-1}$ ). Data points are thinned by a factor of two to more clearly show the wind circulation. The black dot indicates the center position.

Figure 2.2. Fore and aft (horizontal polarization) combined backscatter coefficient ( $\mathrm{dB}$, shaded) and wind vectors are shown for Hurricane Isabel on 10 September, 2003. The black marker within the backscatter weakness and circulation-defined center indicates the center position used in the subsequent low wavenumber analysis. The magenta and blue markers indicate the center positions based on the minimum in backscatter coefficient and best track, respectively....

Figure 2.3. Probability density functions (PDFs) of RMW for all cases are provided, with North Atlantic (NATL, blue), Eastern and Central North Pacific (EPCP, red), Northwest Pacific (WPAC, green), and South Indian and Southwest Pacific (SHEM, 
purple) curves specifically represented. The PDF of RMW determined from SFMR data are also shown for North Atlantic cases (black dashed line).

Figure 2.4. Markers indicate the coincident pairs ( \pm 5 hours) of maximum surface wind speed $\left(\mathrm{m} \mathrm{s}^{-1}\right)$ from SFMR and scatterometer. Shading of the markers indicates the weight applied to the scatterometer swath. A weighted, linear regression fit (dashed line) is compared to the perfect fit (solid line), with the fit equation provided in the legend. The symbol $\delta \mathrm{V}$ indicates that the printed quantity is based on the difference between the paired maximum wind speeds.

Figure 2.5. Azimuthal mean normalized radial profiles of the number of cases (a-b) and the percentage of cases (c-d) for various stratifications within the scatterometer dataset. Panels $(\mathrm{a}, \mathrm{c})$ indicate profiles for all cases and for the specific basins. Panels $(b, d)$ show values for TC intensity. In the top two panels, which are plotted on a logarithmic scale, the dashed gray line indicates the minimum allowable number of cases (50), while the same line in the lower panels indicates $50 \%$ of samples for the respective profile. The total number of cases for each profile is included in the legend.

Figure 2.6. Probability density functions of (a) Shear direction $\left(\theta_{s h r},{ }^{\circ}\right)$, (c) Storm motion direction $\left(\theta_{\text {motion }},{ }^{\circ}\right)$, and (b) Shear - Motion direction $\left(\theta_{s h r^{-}} \theta_{\text {motion }},{ }^{\circ}\right)$, for each individual basin as well as for all basins combined are displayed. The dashed vertical lines in (b) are the angle difference thresholds as discussed in Chen et al. (2006).

Figure 2.7. Composite, normalized wind speed analyses are provided for NATL, EPCP, WPAC, and SHEM in a motion-relative reference frame with and without translation effects in (a-d) and (e-h), respectively. The storm-centered figures are plotted on a radial polar grid using a normalized radius (R/RMW). Contour lines are plotted in increments of 0.025 normalized units, where a value of 1 is equal to the maximum. The blue arrow is the direction of the motion vector and the black marker indicates the location of the maximum wind speed. The maximum wind speed value is also indicated on each panel for reference. Lighter shading indicates areas that do not attain $95 \%$ statistical significance when compared to the global composite

Figure 2.8. Similar to Figure 2.7 but for a shear-relative reference frame. The red arrow indicates the shear direction.

Figure 2.9. Presented similarly to Figure 2.7 but for all basins combined and represented by different $\mathrm{TC}$ intensity groups, where $(\mathrm{a}, \mathrm{d})$ are tropical depressions and storms, $(\mathrm{b}, \mathrm{e})$ are non-major hurricanes, and $(\mathrm{c}, \mathrm{f})$ are major hurricanes. All panels retain translation effects. The top (bottom) row is shown in a motion (shear) relative reference frame.

Figure 2.10. Similar to Figure 2.9 but with translation effects removed. 
Figure 2.11. Presented as a function of latitude ( $\phi$, binned every $\left.10^{\circ}\right)$, PDFs of shear heading and speed and motion heading and speed are displayed in panels (a-b) and (c-d), respectively.

Figure 3.1. In (a), maximum scatterometer wavenumber- $0+1$ amplitudes $\left(V_{s c t}\right)$ are plotted as a function of coincident ( $\pm 5 \mathrm{~h}$ of scatterometer time) maximum SFMR wavenumber- $0+1$ amplitudes $\left(V_{s f m r}\right)$. Varying shades of gray of the markers indicate the weight applied to the scatterometer swath. A weighted linear regression fit is displayed (dashed line) using the weights associated with each marker. In (b), the associated wavenumber-1 phase at the maximum amplitudes are plotted in a similar manner to (a), where the text statements represent the weighted statistical values of the difference between phase angles $\left(\delta \alpha=\alpha_{s f m r}-\alpha_{s c t}\right)$ and the dashed line indicates the weighted linear regression fit.

Figure 3.2. Normalized composite shear-relative (with motion removed) wavenumber- $0+1$, two-dimensional scatterometer wind speed analyses as a function of normalized radius are provided for (a) tropical depressions and storms, (b) Category 1 and 2 hurricanes, and (c) Category 3-5 hurricanes. The red arrows show the direction of shear and the black marker indicates the location of the maximum amplitude. Contours and colors are plotted every 0.025 normalized units. These results are significant at $99 \%$

Figure 3.3. In (a), linear regression fits of the wavenumber-1 phase at the maximum motion-relative amplitude $\left(\alpha_{1}, \max \right)$ are provided for three intensity groups as a function of storm speed $\left(V_{\text {storm }}\right)$. Similar fits are shown in (b) but in a shear-relative (motion removed) reference frame and as a function of vertical wind shear speed $\left(V_{s h r}\right)$. For reference, the zero line is indicative of the down-motion or down-shear direction, with negative phase angles representing locations to the left of motion or shear. The gray markers are the $\alpha_{1}$, max values associated with the tropical depression and storm cases. Dashed lines indicate the $95 \%$ confidence interval of the respective fit.

Figure 3.4. In panels (a-d), TC intensity dependent radial profiles of wavenumber-1 asymmetry ratio $\left(V_{l} / V_{0+1}\right)$ are provided in the form of the four shear-relative quadrant absolute differences that define an asymmetry index (Alvey et al. 2015; Tao et al. 2017). 'DS' and 'US' refer to down-shear and up-shear and the additional 'L' or ' $R$ ' indicates right or left, respectively. In (e), the total asymmetry index is provided similarly to (a-d), where larger values indicate more asymmetry. Dashed lines display the same quantities but for low shear conditions only $\left(V_{s h r}<3.2 \mathrm{~m} \mathrm{~s}^{-1}\right)$.

Figure 3.5. Shear-relative quadrant profiles of surface absolute angular momentum flux $\left(1 \times 10^{6} \mathrm{~m}^{3} \mathrm{~s}^{-2}\right)$ as a function of TC intensity are provided for the full range of shear values (solid lines) and for low shear conditions (dashed lines). The envelopes surrounding the lines represent the $95 \%$ confidence intervals. 
Figure 3.6. For the profiles presented in Figure 3.5, the same quadrant profiles for the individual terms of the absolute angular momentum flux equation are shown. The top and middle rows provide the symmetric and asymmetric relative angular momentum flux (SRAM and ARAM, respectively). The bottom row provides the symmetric Coriolis torque term. The three columns from left to right represent tropical storms, non-major hurricanes, and major hurricanes, respectively, and the colored lines are for the respective shear-relative quadrants.

Figure 3.7. Normalized composite analyses plotted similarly to Figure 3.2 but for angle difference bins denoted as (a) same $\left(\left|\Delta \theta_{s m}\right| \leq 22.5^{\circ}\right)$, (b) left $\left(-157.5^{\circ}<\Delta \theta_{s m}<-\right.$ $\left.22.5^{\circ}\right),(\mathrm{c})$ opposite $\left(\left|\Delta \theta_{s m}\right| \geq 157.5^{\circ}\right)$, and (d) right $\left(22.5^{\circ}<\Delta \theta_{s m}<157.5^{\circ}\right)$.

Figure 3.8. In the left panel, a normalized, bivariate PDF for all cases is shown as a function of $\alpha_{1}$, max and of shear-motion angle differences $\left(\Delta \theta_{s m}\right)$. The right panel shows the same type of PDF but only for Category 1-2 hurricanes. The black line indicates the phase bin with the largest probability of occurrence with errorbars indicating the $95 \%$ confidence intervals.

Figure 3.9. As in Figure 3.4 but for the $\Delta \theta_{s m}$ groups.

Figure 3.10. As in Figure 3.5 but for the $\Delta \theta_{s m}$ groups.

Figure 4.1. Geographical locations of the scatterometer are provided for RI cases with RI-Initial and RI-Continuing explicitly marked.

Figure 4.2. Stacked histograms of (a) RMW, (b) wind shear speed ( $\left.V_{s h r}\right)$, (c) storm motion speed $\left(V_{\text {storm }}\right)$, and $(\mathrm{d})$ shear and motion angle difference $\left(\Delta \theta_{s m}\right)$ are shown for the four intensity change groups (RI, SI, SS, and WK). Values plotted are given as a percentage relative to the total within a particular intensity change group. The text above each bar indicates the percentage of INTS cases within the respective variable bin.

Figure 4.3. Similar to Figure 4.2 but for RI event periods as defined by the RI start $\left(\mathrm{RI}_{0}\right)$ and end $\left(\mathrm{RI}_{\mathrm{E}}\right)$ times. The symbol $t$ refers to the scatterometer time relative to $\mathrm{RI}_{0}$ or $\mathrm{RI}_{\mathrm{E}}$. The text above each bar is the percentage of cases that occur prior to or during the first 12 hours of RI of that variable bin.

Figure 4.4. Linear regression fits of wavenumber-1 phase at the maximum amplitude $\left(\alpha_{1, \max }\right)$ are shown for the four intensity change groups. These are provided as a function of storm speed $\left(V_{\text {storm }}\right)$ without removing motion effects in the left panel and as a function of shear speed $\left(V_{s h r}\right)$ with motion effects removed in the right panel. The fit equations are also included and match the color for their respective line. Dashed lines represent the $95 \%$ confidence interval for each fit.

Figure 4.5. Two dimensional, low wavenumber analyses are provided for each intensity change group as a function of normalized radius (R/RMW). Wind speed 
amplitudes are normalized by the maximum amplitude on a scale from zero to one, where the maximum value is included at the top of the panel. Contours are plotted at intervals of 0.025 normalized units and the gray range rings indicate $2 \times$ and $4 \times \mathrm{RMW}$. The red arrow in each panel indicates the shear direction.

Figure 4.6. Radial profiles of wavenumber-1 asymmetry ratio $\left(V_{l} / V_{0+1}\right)$ are provided in panels (a-d) for four shear-relative quadrant absolute differences as a function of TC intensity change group. These profiles determine the overall asymmetry index, which is provided in (e). 'DS' and 'US' refer to down-shear and up-shear and the additional ' $\mathrm{L}$ ' or ' $\mathrm{R}$ ' indicates right or left, respectively.

Figure 4.7. Absolute angular momentum flux (AAMF, $\left.1 \times 10^{6} \mathrm{~m}^{3} \mathrm{~s}^{-2}\right)$ ) radial profiles are shown with respect to shear-relative quadrants for the intensity change groups. .......93

Figure 4.8. Similar to Figure 4.6 but for RII and RIC composites....................................95

Figure 4.9. Similar to Figure 4.7 but for RII and RIC composites...................................96

Figure 4.10. Presented is the track for Typhoon Parma (WP21, 2003) as taken from the best track data. Solid circles indicate times at 00 UTC while open circles indicate times at 12 UTC. Green triangles indicate center positions from the scatterometer overpasses, and the bold black line indicates the RI portion of the typhoon lifecycle......97

Figure 4.11. In (a), the time series of $V_{\max }$ (black line) from the best track data and RMW as determined from the scatterometer data (gray line) are plotted for Typhoon Parma. In (b), the storm motion (black) and wind shear (gray) speed are plotted, and in (c), their respective directions are shown, where $\theta_{\text {shr }}$ of $0^{\circ}$ (or N) indicates shear directed from south to north. In (d), the difference in the shear and motion angle is provided. Note that the time period is only for 12 hours prior to and after the RI event as denoted in Figure 4.10. Vertical dotted lines in all panels mark the initiation, start of the ending period, and the end of the RI event, respectively. The horizontal dotted lines in (d) represent the angle difference bins as described in KJ17 (Chapter 3)

Figure 4.12. A time-radius plot for (a) normalized wavenumber-0, (b) normalized wavenumber-1 amplitude, (c) wavenumber-1 phase, and (d) wavenumber-1 percentage of the total amplitude is shown for the Typhoon Parma RI event. The three dashed lines mark the same RI-relevant periods as noted in Figure 4.11.

Figure 4.13. Similar to Figure 4.11 but for the composite RI event timeline. Values plotted represent medians for the respective variables.

Figure 4.14. Similar to Figure 4.6 but for the designated RI event periods. 103

Figure 4.15. Similar to Figure 4.12 but for the composite RI event progression. 
Figure 4.16. A time-radius plot of the gradient of AAM flux is provided for the composite RI event times. The solid black line is the location of the maximum AAM flux gradient for a particular time. The dashed lines represent the times over which RI occurs.

Figure 4.17. Time-radius images are shown for (a) the azimuthal difference between the maximum low wavenumber amplitude and maximum TMI rain rate, and (b) the normalized radius difference between the maximum wind and rain parameters in (a). Negative values in (a) indicate the low wavenumber phase is downwind of the maximum rain rate azimuth while negative values in (b) indicate a radial location of the maximum rain rate outward of the surface wind radius. The slight difference in time labels compared to Figure 4.16 is due to the constraints in the TMI dataset.

Figure 5.1. In the left panel, the three nested grids for the 2015 version of HWRF are provided for the outer (red), middle (green), and inner (blue) domains. The inset panel provides a magnified view of the two inner domains, where dashed rings indicate radial distances of 50, 150, 250, and $500 \mathrm{~km}$ from the TC center.

Figure 5.2. In (a), the paired samples of best track and scatterometer (blue), SFMR (red), and Dvorak (green) intensity estimates are provided. Model results (in gray) are evaluated against the model determined maximum $10-\mathrm{m}$ wind speed at a given forecast time. Solid lines indicate the linear regression fit of the data with coefficient of determination values indicated by matching colored text. In (b), the residual PDFs of the scatterometer, SFMR, and model low wavenumber data are provided. A PDF of the Dvorak differences is also shown but is not representative of the epsilon term. Mean $(\mu)$, standard deviation $(\sigma)$, and sample size $(n)$ are indicated as well as the average uncertainty from Landsea and Franklin (2013, black vertical line).

Figure 5.3. Cumulative distribution functions (CDFs) of $\varepsilon$ are provided for model, SFMR, and scatterometer data. The CDF for the Dvorak intensity difference is also included. Colors are the same as in Figure 5.2.

Figure 5.4. In (a), PDFs of the Dvorak difference with best track $V_{\max }$ are shown for all cases and for each basin. The matching colored text indicates mean and standard deviation. Similarly to (a), panel (b) provides PDFs of $\varepsilon$ for the scatterometer data. The black vertical line is representative of the best track intensity uncertainty.....

Figure 5.5. Similarly to Figure 5.4(b), PDFs of $\varepsilon$ are separated by storm intensity for (a) tropical storms, (b) Category 1-2 hurricanes, and (c) Category 3-5 hurricanes and are provided for model (gray), SFMR (red), and scatterometer (blue) data. Mean, standard deviation, and sample size are indicated by the text.

Figure 5.6. Similar to Figure 5.5, PDFs of $\varepsilon$ are separated by intensity change groups for (a) rapidly intensifying, (b) slowly intensifying, (c) steady state, and (d) weakening TCs. 
Figure 5.7. Three successive HWRF simulations Hurricane Irwin (11E) are shown and include the best track $V_{\max }$ (solid blue line), model $\mathrm{V}_{\max }$ and $\varepsilon$ (black solid and dashed lines, respectively) and scatterometer $\varepsilon$ (blue dashed line) as a function of forecast hour. The combined $\left(V_{0+1, \max }\right)$ and individual low wavenumber amplitudes $\left(V_{0, \max }\right.$ and $\left.V_{l, \max }\right)$ are provided for model (gray solid, dashed, and dash-dot lines respectively) and scatterometer (red solid, dashed, and dash-dot lines) data.

Figure 5.8. Similar to Figure 5.7. but for Hurricane Katia (12L).................................. 135

Figure 5.9. In (a), mean error of the forecast value compared to the same variable from the scatterometer data is shown as a function of forecast hour for $V_{0}$ (blue), $V_{l}$ (red), $\varepsilon$ (green), and $V_{\max }$ (purple). The number of matching forecast cases is provided at each 6-hr forecast time. In (b), similar lines are provided as in (a) but for the mean absolute error. The cases considered in these comparisons include only TCs below major hurricane strength.

Figure 5.10. Displayed is a normalized joint PDF of the rate of intensity change and the wavenumber-1 phase difference with the azimuthal location of the maximum 10$\mathrm{m}$ rain rate as determined from the model forecast data $(\Delta \phi)$. Warmer colors indicate higher probabilities and the black markers represent the azimuthal difference bin with the highest probability for each intensity change bin. The horizontal error bars indicate the $95 \%$ confidence interval. Negative angles indicate that $\alpha_{1, \max }$ is downwind of $\phi_{r r, \max }$, where DWF and DWR refer to the downwind forward and rear quadrants.

Figure 5.11 Similar to Fig 5.10 but for the designated RI event time periods. $\mathrm{RI}_{0}$ and $\mathrm{RI}_{\mathrm{E}}$ indicate $\mathrm{RI}$ initial and ending times, respectively. 


\section{LIST OF ACRONYMS AND ABBREVIATIONS}

AAM

ACT

AEJ

AEW

AL

ARAM

ASCAT

ATCF

CAT $1-2$

CAT 3-5

CB

CDF

CI

CISK

$\mathrm{DM}$

DS

DW

EP

EPCP

ERC

FA

FCHLP

GFDL

GFS

GPS
Absolute Angular Momentum

Asymmetric Coriolis Torque

African Easterly Jet

African Easterly Wave

North Atlantic (for ATCF storm numbers)

Asymmetric Relative Absolute Angular Momentum Flux

The Advanced Scatterometer

Automated Tropical Cyclone Forecasting

Category 1-2 hurricane

Category 3-5 hurricane

Convective burst

Cumulative Distribution Function

Current Intensity

Conditional Instability of a Second Kind

Down-Motion (can add ' $L$ ' or ' $R$ ' for left or right; ' $U$ ' substitutes with ' $\mathrm{D}$ ' to signify up)

Down-Shear (add ' $L$ ' or ' $R$ ' for left or right; ' $U$ ' substitutes with ' $\mathrm{D}$ ' to signify up)

Down-Wind (add ' $F$ ' or ' $R$ ' for forward or rear; ' $U$ ' substitutes with ' $\mathrm{D}$ ' to signify up)

Eastern Pacific (for ATCF storm numbers)

Eastern and Central North Pacific ocean basin

Eyewall Replacement Cycle

Ferrier-Aligo microphysics scheme

Florida Commission on Hurricane Loss Projection

Geophysical Fluid Dynamics Laboratory

Global Forecast System

Global Positioning System 


\begin{tabular}{ll} 
HFIP & Hurricane Forecast Improvement Project \\
HURDAT2 & Hurricane Database 2 \\
HWRF & Hurricane Weather Research and Forecasting model \\
IFEX & Intensity Forecast Experiment \\
INTS & Intensifying Tropical Cyclone \\
ITCZ & Inter Tropical Convergence Zone \\
ITOP & Impact of Typhoons on the Ocean in the Pacific \\
JPL & Jet Propulsion Laboratory \\
JTWC & Joint Typhoon Warning Center \\
MAE & Mean Absolute Error \\
MCS & Mesoscale Convective System \\
NASA & National Aeronautics and Space Agency \\
NATL & North Atlantic Ocean basin \\
NHC & National Hurricane Center \\
NHRP & National Hurricane Research Project \\
NINTS & Non-Intensifying Tropical Cyclone \\
NMM & Nonhydrostatic Mesoscale Model \\
NOAA & National Oceanic and Atmospheric Administration \\
NN & Neural Network \\
OSCAT & OceanSat-2 Scatterometer \\
PDF & Probability Density Function \\
QuikSCAT & Sea-Winds Scatterometer \\
RI & Rapid Intensification \\
RIC & RI-Continuing \\
RII & RI-Initial \\
RMW & Radius of Maximum Winds \\
RMSE & Root-Mean Squared Error \\
RR & Rain rate \\
RRTMG & Rapid Radiative Transfer for General Circulation Models \\
RTM & Radiative Transfer Model \\
\hline
\end{tabular}




\begin{tabular}{ll} 
RW & Rapid Weakening \\
SAS & Simplified Arakawa Schubert scheme \\
SCT & Symmetric Coriolis Torque \\
SFMR & Stepped Frequency Microwave Radiometer \\
SHEM & Southern Hemisphere \\
SHIPS & Statistical Hurricane Intensity Prediction Scheme \\
SI & Slow Intensification \\
SIDX & Symmetry Index \\
SRAM & Symmetric Relative Absolute Angular Momentum Flux \\
SS & Steady State \\
SST & Sea Surface Temperature \\
SSWS & Saffir-Simpson Wind Scale \\
SW & Slow Weakening \\
TB & Brightness Temperature \\
TC & Tropical Cyclone \\
TCIS & Tropical Cyclone Information System \\
TCS-08 & Tropical Cyclone Structure 2008 \\
TD & Tropical Depression \\
TMI & TRMM Microwave Imager \\
TRMM & Tropical Rainfall Measuring Mission \\
TS & Tropical Storm \\
VHT & Vortical Hot Tower \\
WISHE & Wind-Induced Surface Heat Exchange \\
WK & Weakening Tropical Cyclone \\
WRF-ARW & Advanced Research Weather Research and Forecasting \\
& model \\
& \\
\hline
\end{tabular}




\section{INTRODUCTION}

\subsection{Background and Motivation of Work}

Tropical cyclones (TCs) are complex, rotating vortices that regularly form over the Earth's warm, tropical ocean waters and often threaten, disrupt, or completely devastate coastal communities. Records of TCs date back nearly 1,000 years (Emanuel 2005), but the science of understanding the processes that control their formation and intensification has grown significantly during the past century. Lorenz (1983) in his summary of the general circulation of the atmosphere notes of a cyclical pattern where a theory about a certain process is assumed true until improvements in the theory or observation increase uncertainty in said process. In turn, this uncertainty motivates new ideas and technologies that aid scientific innovation and progression. In regards to TCs, this premise holds true as well. With advancements in aircraft reconnaissance and the inception and improvement of satellite observations over the past few decades, it is possible to continuously monitor the progression of a $\mathrm{TC}$ and provide notifications and warnings to affected populations. However, the turbulent and often increasingly chaotic nature of meso- and small-scale processes within TCs combined with interactions of the large-scale environment make understanding and predicting these systems difficult.

Tackling the TC problem is currently a multi-agency and international undertaking with the intent of not only improving the understanding of TC processes but also improving the ability to more accurately predict their development. The ultimate goal is to provide people with a better opportunity to protect themselves, their families, and their property against the impacts of TCs. Because those affected by TCs experience processes that occur near the surface, it is important that a better grasp of these processes 
is obtained. Specifically, it is important to understand surface wind structure in relation to environmental impacts and coincident internal components. Therefore, this dissertation explains the variability of surface wind structure from an observational perspective and provides an analysis of the predictability and verification capabilities of this newfound knowledge. Prior to discussing the results of the study, it is important to describe the current knowledge of TCs, and the following sections describe 1) the general three-

dimensional TC structure, 2) formation and intensification theories, 3) observational techniques, and 4) current prediction capabilities. A final section offers some expectations of this study.

\subsection{Summary of Tropical Cyclones}

\subsubsection{General Structure}

According to the National Hurricane Center (NHC), a tropical cyclone is described as "a warm-core, non-frontal synoptic-scale rotational vortex, originating over tropical or subtropical waters, with organized deep convection and a closed surface wind circulation about a well-defined center." The definition includes four important and cooperative processes for TC maintenance and intensification, including kinematic, thermodynamic, precipitative, and oceanic contributions. Because TCs are generally referenced by their intensity (tropical storm or hurricane), the first process to discuss is the hurricane winds. While the definition speaks of the surface circulation, the TC is a three-dimensional entity with horizontally rotating winds throughout the vertical column. These horizontal rotating winds are generally referred to as the primary $\mathrm{TC}$ circulation (i.e., Willoughby 1988 and references therein). The swirling winds, which rotate counterclockwise around a low-pressure center in the Northern Hemisphere, are a result of a 
balance of the pressure gradient force (inward) and the centrifugal and Coriolis forces (outward) in a pure gradient balance sense. Figure 1.1 taken from Smith (2006) provides a schematic diagram of this ideal primary circulation.

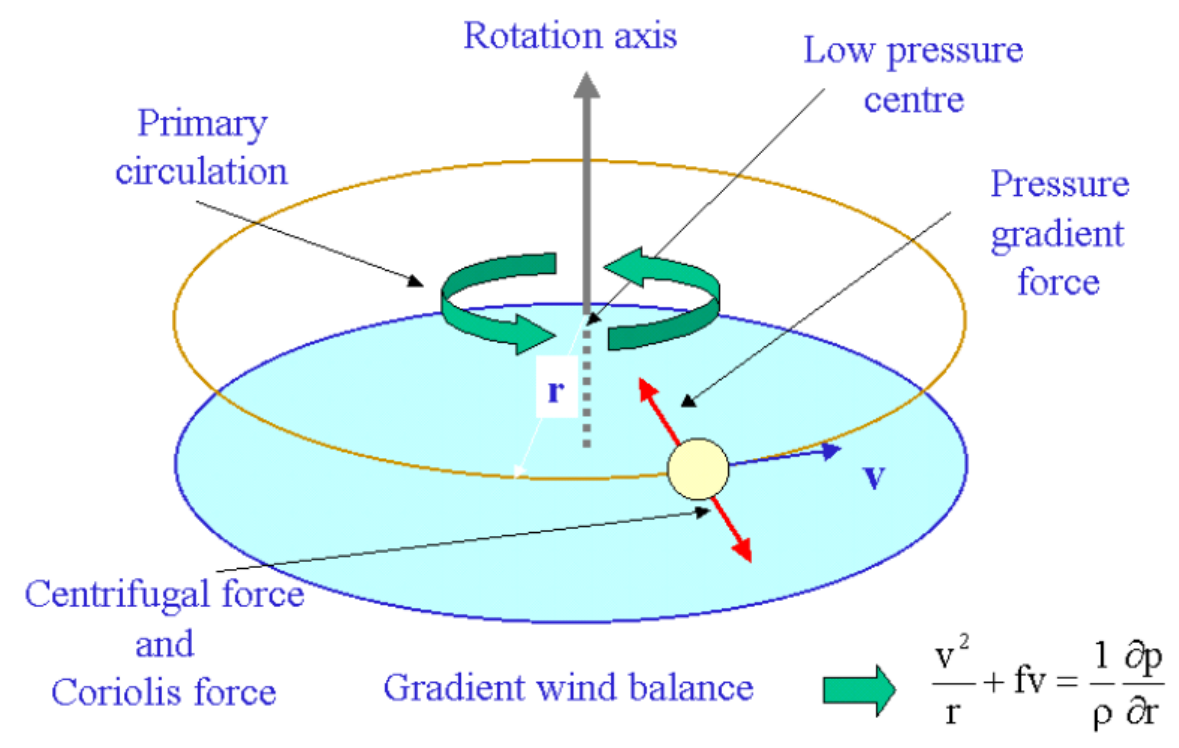

Figure 1.1. Taken from Smith (2006), this schematic diagram shows the ideal primary TC circulation in gradient balance with respective forces indicated with the text.

Willoughby (1990) indicates that this gradient balance is a fairly accurate depiction of the circulation above the TC boundary layer ( 1-2 km above the surface), but because of increasing impacts from friction near the surface, the low level primary circulation has an inward component that promotes the development of an eyewall around a central and often clear eye that forms from subsiding air aloft. The maximum horizontal winds form near the eyewall as a response to the strong pressure gradient. The convergence of moist and high momentum air near the surface also promotes a secondary circulation upward through the vertical column (Jorgensen 1984; Willoughby 1988). Once the air emerges near the top of the TC, it is moved outward from the center in response to the formation of an upper-level, high-pressure region. The strength of the 
secondary circulation becomes more apparent with increasing strength of a TC. Figure 1.2 provides an example of the secondary TC circulation (Willoughby 1988).

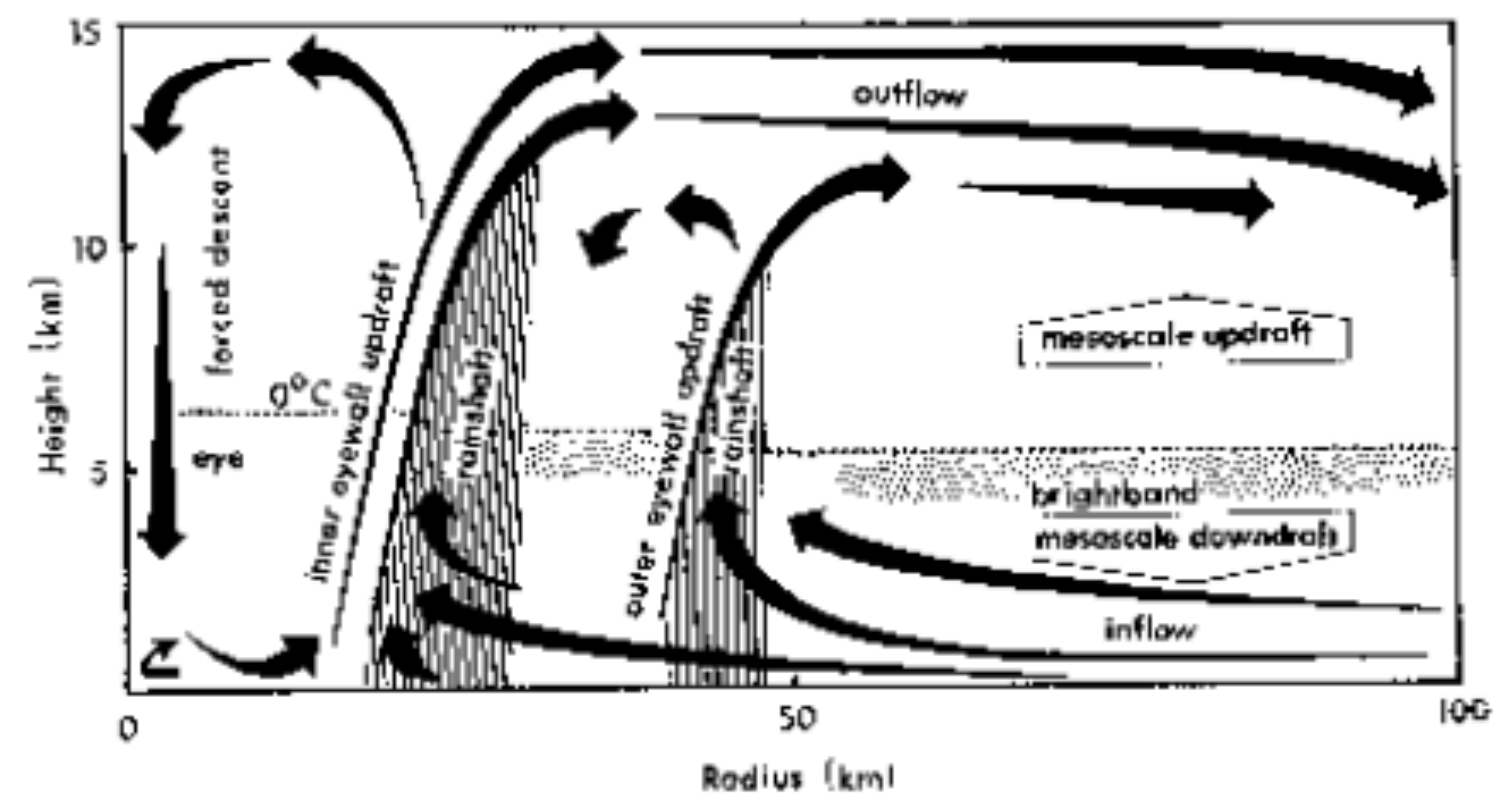

Figure 1.2. The schematic diagram of the secondary circulation of a mature tropical cyclone is displayed [taken from Willoughby (1988)]. Black arrows indicate the air flow direction.

The low-level inflowing air also imports an abundance of moisture as a result of interaction with the ocean surface, which generally must exceed sea-surface temperatures (SSTs) of $26^{\circ} \mathrm{C}$ (Gray 1968; 1979) to be a significant source of energy. As the moist air spirals inward toward the TC center and is forced upward, the air cools and produces rain. In the process, latent heat is released radially inside the eyewall. Because adiabatic cooling does not fully offset the latent heat release, the process acts to enhance the upper level warm core of the TC (Malkus and Riehl 1960; Charney and Eliassen 1964; Schubert and Hack 1982; Shapiro and Willoughby 1982). Early aircraft reconnaissance within mature hurricanes confirms the presence of the warm core and subsiding air in the eye (La Seur and Hawkins 1964; Hawkins and Rubsam 1968). Figure 1.3, which is adapted 
from Hawkins and Rubsam (1968), provides an example of the warm core from Hurricane Hilda in 1964.

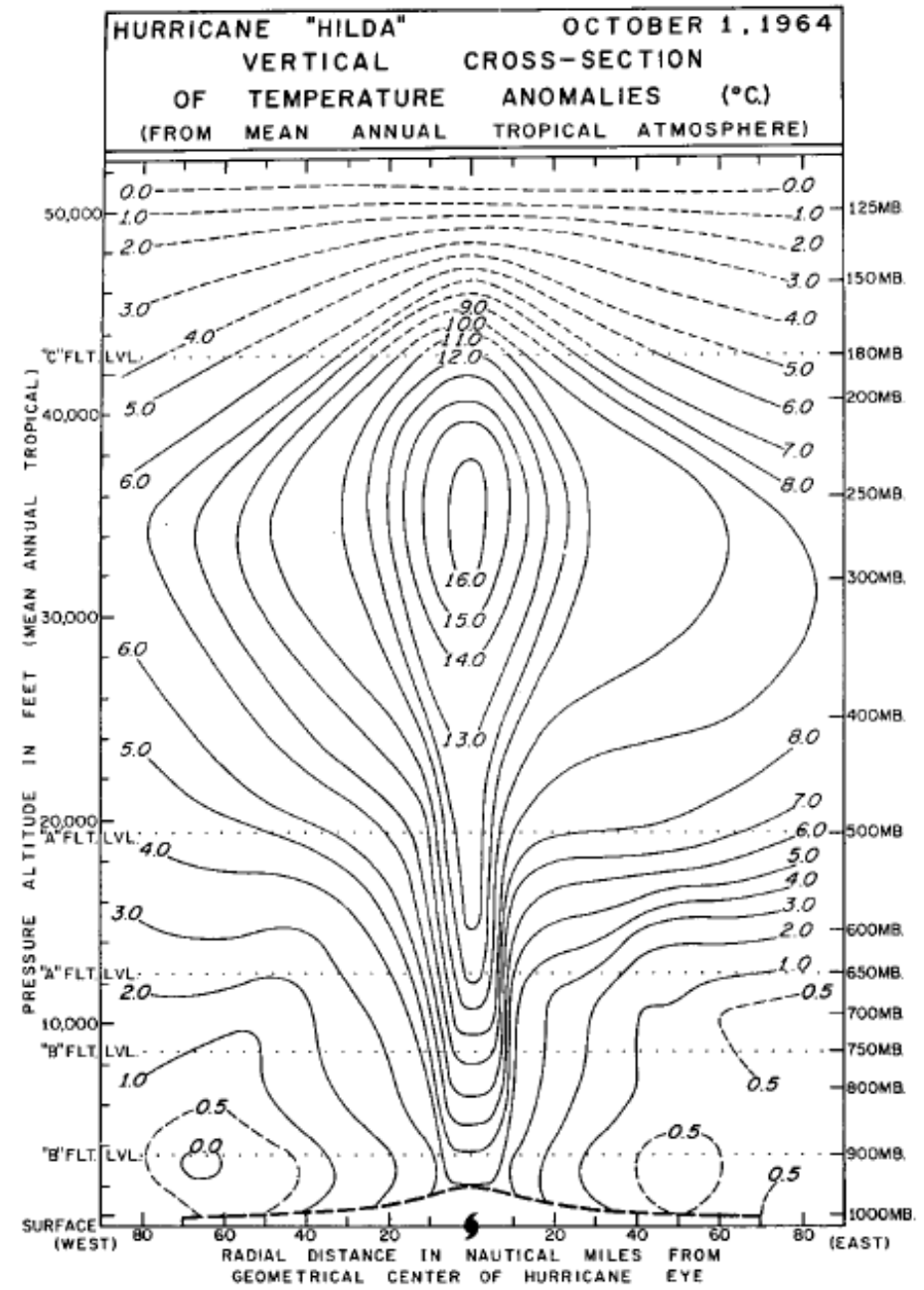

Figure 1.3. From Hawkins and Rubsam (1968), the vertical temperature anomaly from Hurricane Hilda (1964) is shown. Anomalies are plotted in increments of $1{ }^{\circ} \mathrm{C}$.

The final main component of the tropical cyclone structure is related to convection and precipitation. The upward motion induced by convergence of the moist, low-level air produces the main region of convection in the TC eyewall. Willoughby et al. (1984) note that there are usually several circular rings of convection within $\sim 100 \mathrm{~km}$ of the TC center that locally generate latent heat release and move inward, where outer rings often replace inner rings in what is often referred to as an eyewall replacement 
cycle (ERC, Willoughby et al. 1982). These ERCs usually occur in very strong hurricanes, while less intense or asymmetric TCs usually have spiral-like bands or rainbands that form radially outward of the eyewall. Figure 1.4 provides a schematic diagram from Willoughby (1988) that describes the general convective and precipitation structure in hurricanes. Notice that in this radar-derived image, the rainbands tend to consist of intermittent convective cells whereas the eyewall contains a dense area of heavy rain associated with deep convection.

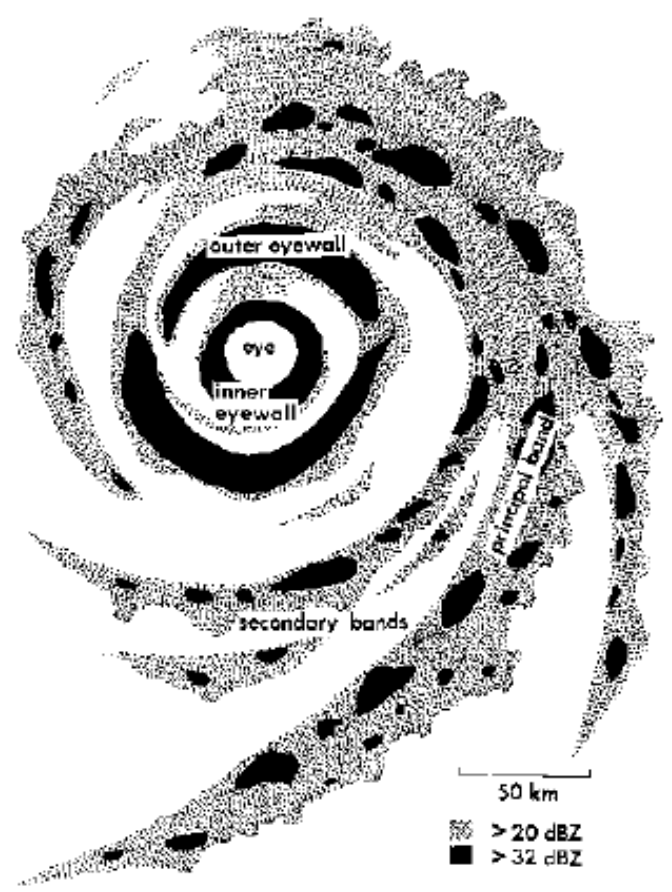

Figure 1.4. A schematic diagram derived from airborne radar depicts the main characteristics of the hurricane convection and precipitation [taken from Willoughby (1988)].

Looking in the vertical, the convection in the eyewall tends to tilt outward from the center in response to outward tilting angular momentum surface (Malkus and Riehl, 1960; Jorgensen 1984). As the winds above the boundary layer begin to move away from the TC center, sinking motion produces clear regions between rainbands. Additionally, 
local downdrafts associated with rainband convection produce large regions of stratiform rain around the hurricane vortex. Jorgensen (1984) developed a general schematic of the vertical convective structure in hurricanes, and Figure 1.5 shows his result. According to this discussion, a mature TC is multifaceted and the processes feed back upon each other. If one or more of these processes is disrupted, the feedback between processes limits the ability of a TC to strengthen.

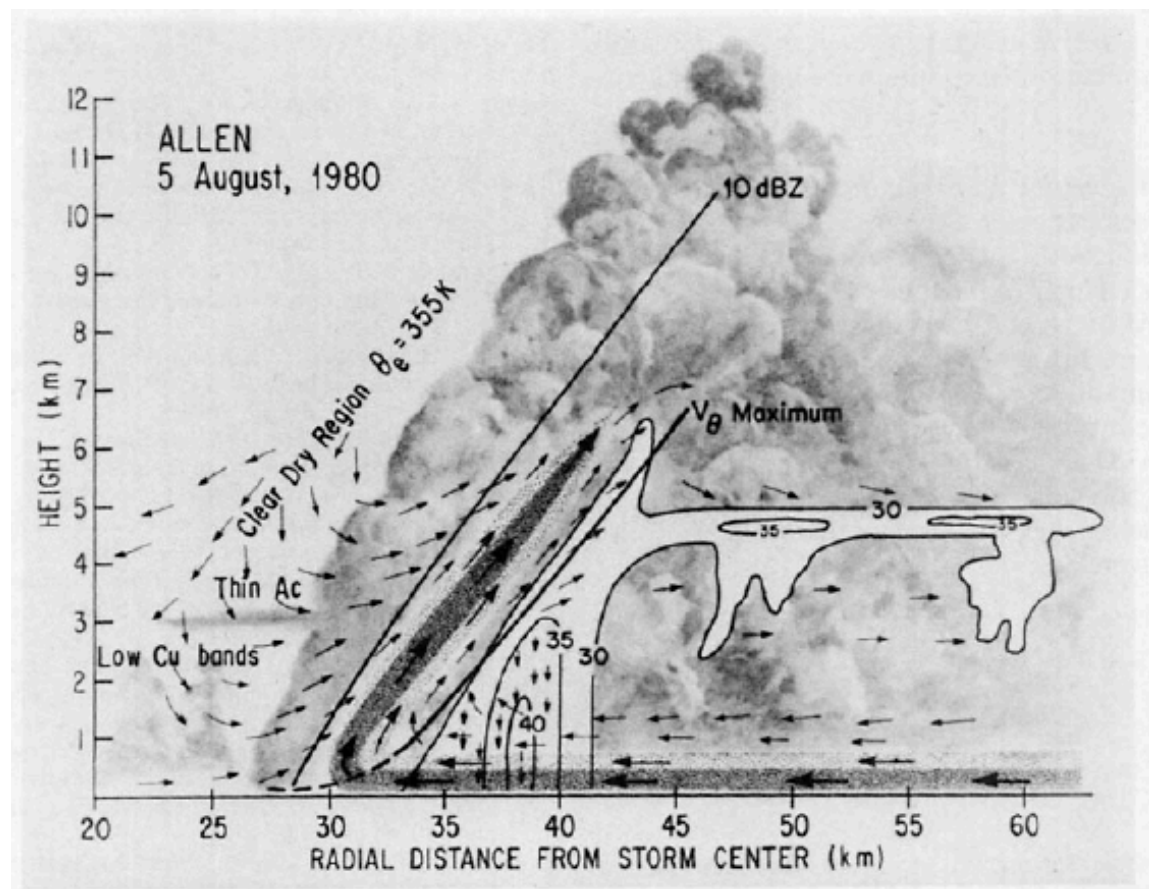

Figure 1.5. Adapted from Jorgensen (1984), a cross-section schematic of the expected convective structure of a mature hurricane is shown. Data for this display was gathered from radar data collected in Hurricane Allen (1980).

\subsubsection{Formation and Intensification}

Despite the knowledge of mature TC structure, there is still debate on how these destructive storms form. Gray $(1968 ; 1979)$ provides an early discourse on the ingredients needed for TC formation, including: an existing area of disturbed weather with sufficient low-level convergence, warm ocean waters (SST $\geq 26{ }^{\circ} \mathrm{C}$ ), a moist 
atmosphere that is potentially unstable to allow growth of convection, and weak vertical wind shear. The presence of strong wind shear, which in this sense is represented by a wind difference between the upper and lower troposphere, disrupts the organization of convection around the rotating vortex.

These main variables are necessary for TC development, but $<50 \%$ of cases of disturbed weather in the tropics meeting these requirements develop into TCs (i.e., Hopsch et al. 2007). Before discussing the processes involved in pushing the disturbance towards formation, it is important to note the different sources of these disturbances for the world's TC formation regions. For the North Atlantic, entities known as African Easterly Waves (AEWs, Burpee 1972; Thorncroft and Hodges 2001) develop over the Sahel region of Africa in response to a temperature gradient between the Sahara desert and the moist region to the south over the Guinea highlands (Fontaine et al. 1995). Mesoscale convective systems (MCSs) form as squall lines in the presence of a mid-level $(600 \mathrm{hPa})$ African Easterly Jet (AEJ) and propagate westward. If the components of the AEW are sufficient, they can ultimately form into a TC over the ocean (Klotz and Kucera 2012). This source mostly applies to the Atlantic basin. For the other global basins, the main source of disturbed weather originates in the Intertropical Convergence Zone (ITCZ). Marked as a band of thunderstorm activity that has a semiannual track across the equator, the ITCZ can sometimes invigorate long-lived convection that moves poleward and forms into a TC. In conjunction with the ITCZ, the Western North Pacific is under the influence of a large area of warm SSTs, often called the Western Pacific warm pool. The presence of sufficiently warm SSTs enhances the opportunity of ITCZ or other tropical convection to organize and develop into a TC. Because of this warm pool in the 
ocean, the Western North Pacific generally experiences the longest TC season and usually has the highest number of developing TCs in a given year (i.e., Chan and Shi 1996; Emanuel 2005).

As stated previously, the presence of convection in the tropics does not automatically necessitate TC formation. An early theory for TC formation and intensification discussed in Charney and Eliassen (1964) is described as combination of conditional instability from cumulus development and low-level convergence from an existing pressure gradient. Referred to as conditional instability of a second kind (CISK), the technique was verified using a revolutionary numerical-dynamical model that adequately reproduced the formation of a mature hurricane (Ooyama 1969). Because the tropical cyclone development is dependent on a warm core, Schubert and Hack (1982) further advanced the idea of inertial instability increasing as a response to an increased rate of heating near the TC core.

Emanuel (1986) and Rotunno and Emanuel (1987) suggest a modification to the CISK theory, which they state relies too heavily on convective processes and does not account for imbalance of equivalent potential temperature in the boundary layer. Termed wind-induced surface heat exchange (WISHE), Rotunno and Emanuel (1987) indicate that the TC formation and intensification is a result of air-sea fluxes arising from the lowlevel inflow, which then induces the secondary circulation. Numerical simulations by Craig and Gray (1996) tend to support formation through WISHE, but they note there is significant variability in their results.

A more recent theory of TC formation and intensification suggested by Nguyen et al. (2008) and Montgomery et al. (2009) reverts somewhat to ideas originally suggested 
by Riehl and Malkus (1958), where asymmetric 'vortical hot towers' (VHTs) pulse highly stretched and rotating convective clouds into an already large-scale vortical structure. Over time, the axisymmetrization of these towers in conjunction with their lowlevel convergence leads to the development and intensification of a TC in their model. The authors suggest that this method more accurately depicts the relative role of convection and air-sea moisture fluxes in TC intensification, essentially indicating WISHE is not truly representative of the three-dimensional intensification process. Montgomery et al. (2015) also indicates that the WISHE method, while correct in its reliance on air-sea interaction, should not be considered the dominant mode of TC intensification because of shortcomings of the inferred reference to a simplified, closed Carnot cycle.

\subsection{Observing Tropical Cyclones and Their Surface Winds}

Despite the uncertainty in the exact path of TC formation, these storms exhibit the common characteristic of having a maximum wind speed in the vicinity of the eyewall. However, the distance from the center is dependent on the pressure gradient, size of the storm, and the current strength of the TC. Forecast centers around the world have requirements for estimating a maximum surface wind speed within a TC, which for the North Atlantic and Eastern North Pacific is defined by a 1-minute average, sustained wind speed (Jarvinen et al. 1984). Maximum surface winds $\left(V_{\max }\right)$ are classified as a tropical depression $\left(V_{\max }=15 \mathrm{~m} \mathrm{~s}^{-1}\right)$, tropical storm $\left(17 \leq V_{\max }<33 \mathrm{~m} \mathrm{~s}^{-1}\right)$, hurricane (33 $\left.\leq V_{\max }<50 \mathrm{~m} \mathrm{~s}^{-1}\right)$, or a major hurricane $\left(V_{\max } \geq 50 \mathrm{~m} \mathrm{~s}^{-1}\right)$. Hurricanes are further separated into Category 1-5 designations according to the Saffir-Simpson wind scale (SSWS, Simpson and Riehl 1981). Table 1.1 below provides the SSWS designations as 
indicated in Simpson and Riehl (1981). Surface observations from land-based stations are helpful for updating conditions when a TC makes landfall, but it is most important for forecasters and decision makers to obtain information well before a TC impacts land. Surface wind observations are thus obtained from two main sources: satellite-based or insitu aircraft reconnaissance. The latter is described first.

Table 1.1. Included are the Saffir-Simpson hurricane wind classifications (modified from Simpson and Riehl 1981). Note that wind speeds are in miles per hour (mph), where 1 $\mathrm{mph}=0.869 \mathrm{kt}$ or $0.447 \mathrm{~m} \mathrm{~s}^{-1}$.

\begin{tabular}{|c|c|c|c|c|}
\hline $\begin{array}{c}\text { Scale Number } \\
\text { (category) }\end{array}$ & $\begin{array}{c}\text { Central Pressure } \\
(\mathrm{mb})\end{array}$ & $\begin{array}{c}\text { Winds } \\
(\mathrm{mph})\end{array}$ & $\begin{array}{c}\text { Surge } \\
(\mathrm{ft})\end{array}$ & Damage \\
\hline 1 & $\geq 980$ & $74-95$ & $4-5$ & Minimal \\
\hline 2 & $965-979$ & $96-110$ & $6-8$ & Moderate \\
\hline 3 & $945-964$ & $111-130$ & $9-12$ & Extensive \\
\hline 4 & $920-944$ & $131-155$ & $13-18$ & Extreme \\
\hline 5 & $<920$ & $>155$ & $>18$ & Catastrophic \\
\hline
\end{tabular}

\subsubsection{Aircraft Reconnaissance}

Observations of TCs obtained from research and operational aircraft are generally trusted as the best source of structural information. Some form of aircraft reconnaissance into TCs has existed since the mid-to-late 1940s. Through the 1950s and 60s, United States Navy pilots flew missions into typhoons in the Western North Pacific, but the missions were intermittent at best. Sparked by several devastating hurricanes striking the mid-Atlantic and New England coasts of the United States, the Weather Bureau was tasked with forming the National Hurricane Research Project (NHRP, Aberson et al. 2006 and references therein). Meteorological observing technology was in its infancy at the time, however, limiting the quality of the information provided during these missions. In the mid-1970s, the National Oceanic and Atmospheric Administration (NOAA) 
obtained two Navy WP-3D (or P-3) aircraft, and they have remained the premiere research platform for collecting data in TCs and other meteorological phenomena.

The two NOAA P-3s are presently equipped with instrumentation that record information regarding TC winds, temperature, moisture, and precipitation. For the purposes of this study, the focus is placed on the surface wind information. Each aircraft is fitted with a stepped frequency microwave radiometer (SFMR), which is a passive microwave instrument that uses a radiative transfer model (RTM) to obtain a surface brightness temperature $\left(T_{B}\right)$. This brightness temperature is then converted into a surface wind speed and rain rate estimate. Figure 1.6, which is taken from Uhlhorn and Black (2003), provides a graphic of the RTM components. Dependent on atmospheric and oceanic parameters, the SFMR uses six C-band frequencies in a stepping manner to obtain the most accurate representation of the $\mathrm{T}_{\mathrm{B}}$ and wind speed.

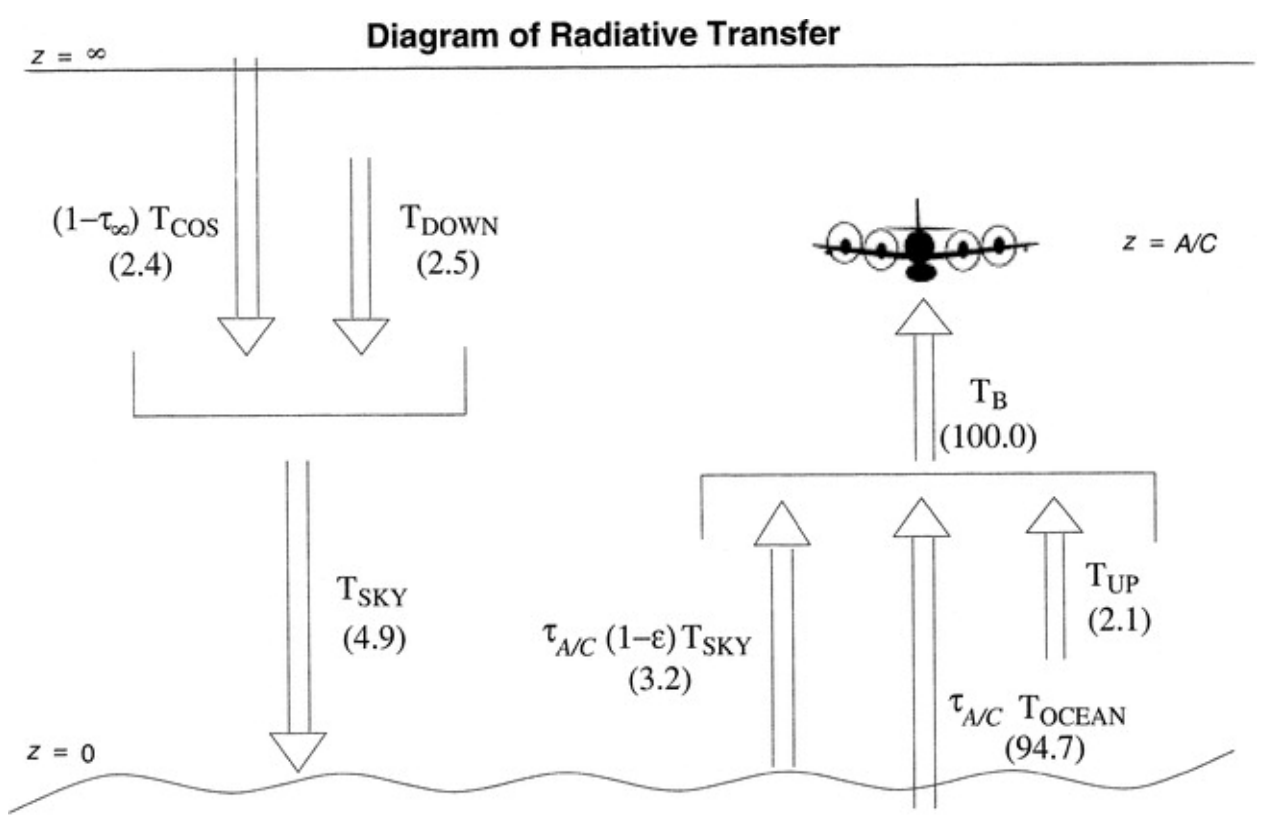

Figure 1.6. From Uhlhorn and Black (2003), the radiative transfer schematic diagram is provided for the airborne stepped frequency microwave radiometer (SFMR). 
The first recorded measurements by an SFMR were in Hurricane Allen in 1980 (Uhlhorn and Black 2003), but surface winds were not provided operationally until 1999. Through the present time, SFMR data are available for many TCs, and these data are considered the best available estimate of surface wind speeds in hurricanes and the standard for intensity estimation and verification. As with any observing system, SFMR is not without its shortcomings. Because it is a C-band (4-7 GHz) radiometer, interference from rain is known to cause an overestimation of weak wind speeds $(<33 \mathrm{~m}$ $\left.\mathrm{s}^{-1}\right)$. Recent advancements in correcting for rain prompted an improvement in the SFMR wind speed estimate (Klotz and Uhlhorn 2014). Another drawback for SFMR is related to the flight patterns chosen for data collection. Often using a form of an "alpha" or "figure4" pattern (i.e., Uhlhorn and Nolan 2012), wind speed and rain rate are densely collected in a radial sense but sparsely in an azimuthal sense. The lack of full storm coverage increases the uncertainty of the maximum surface winds. Uhlhorn and Nolan note that following the standard observation method produces an average underestimate of the maximum wind speed by $\sim 8-10 \%$.

\subsubsection{Observations from Satellite-based Instruments: Scatterometry}

As part of the technological revolution starting in the 1960s and early 1970s, meteorological satellites were placed in Earth's orbit as unique observing systems that could provide: 1) a basic coverage of TC progression and 2) primitive TC intensity estimates in regions that aircraft could not reach. Further advancements to spaceborne radars, atmospheric profilers, radiometers, and microwave instrumentation have afforded the opportunity to study TCs on a global scale in all aspects of their structure (kinematic, thermodynamic, and precipitative). 
Observing surface conditions within a TC from spaceborne instruments was understandably difficult at the time because of attenuation of the transmitted signal from rain and ice. However, development and operation of a scatterometer in the mid-1970s proved that surface winds were observable from space with known limitations. Scatterometer observing techniques use proven radar methods, where a signal (either $\mathrm{Ku}-$ or C-band) is transmitted to the ocean surface, and a backscattered return signal is converted to a wind speed estimate using a RTM. From variations in return signal, scatterometers are also able to provide a wind direction, unlike SFMR. Success of a short-lived, dual-swath scatterometer (the first of its kind) operated by the National Aeronautics and Space Agency (NASA) known as NSCAT prompted the production and launch of a longer-lived and successful scatterometer known as SeaWinds on board the QuikSCAT satellite in 1999. Taken from Spencer et al. (1997), Figure 1.7 shows the scanning geometry of the SeaWinds dual-swath scatterometer.

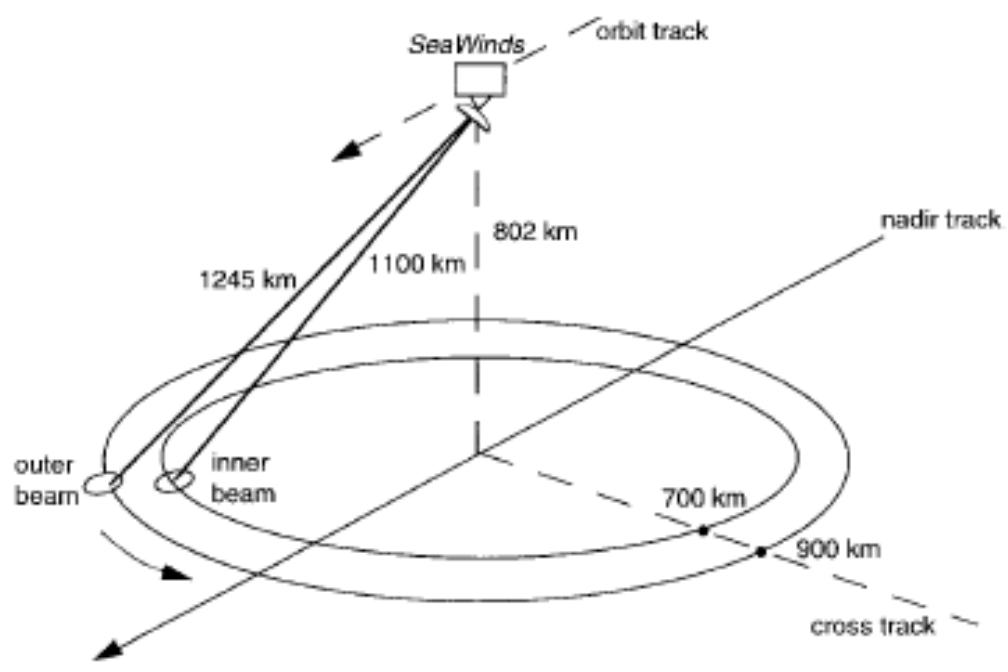

Figure 1.7. The scanning geometry of the QuikSCAT scatterometer is provided (from Spencer et al. 1997). 
While still limited in terms of its ability to accurately depict surface wind speeds in raining conditions, the QuikSCAT scatterometer (and scatterometers in general) offer several advantages over aircraft reconnaissance. Unlike aircraft that are dependent on range from a base of operations, scatterometers are consistently measuring ocean surface conditions and providing global coverage of surface winds. The swath of data collected by a scatterometer also has the ability to fully observe the TC surface wind field at a given time, making it especially useful for performing TC size climatology studies and evaluating operational wind radii as necessitated by operational forecasting centers (Brennan et al. 2009; Chavas and Emanuel 2010; Chavas et al. 2016). The scatterometer scanning technique and orbital parameters are limiting in several aspects, including: 1) inability to target a $\mathrm{TC}, 2)$ reduced radial coverage due to horizontal resolution constraints, and 3) inability to consistently determine wind speeds in the TC inner core due to rain attenuation. A recent study by Stiles et al. (2014) has addressed item 3) to a certain extent, making post-processed scatterometer data useful for evaluating the entire TC surface wind field.

\subsection{The State of Tropical Cyclone Forecasting}

Because my study uses information provided in operational forecasts as well as forecast models, a brief description of the current state of TC forecasting is subsequently presented. Forecast centers are required by their respective governments with the assistance of the World Meteorological Organization (WMO) to provide current TC intensity and position to their constituents along with a forecast of future position and strength. At NHC, hurricane specialists use all available observational products to assess the current state of a TC. They also use their experience along with guidance from 
numerically or statistically driven TC models to develop future forecast position and intensity. After surveying a group of hurricane forecasters at NHC, Landsea and Franklin (2013) indicate significant improvements in their subjectively obtained TC position uncertainty since the early 1990s. For initial forecast times, the average position uncertainty is $\sim 37 \mathrm{~km}$. For 24 hour forecasts, their position estimate improved by $\sim 93 \mathrm{~km}$ ( $\sim 100 \%$ improvement) between 1990 and 2011 , and at 72 hour forecasts, position estimates improved by over $370 \mathrm{~km}$ ( $>200 \%$ improvement). Similar assessments of their intensity forecasts conversely show little to no improvement over the same 20 -year period.

Similar intensity and position uncertainties were also present in the numerical guidance provided to NHC, suggesting that $\mathrm{TC}$ model configuration related to grid resolution and simulated processes did not adequately reproduce processes observed in nature. In 2008, NOAA approved a 10-year program named the Hurricane Forecast Improvement Project (HFIP) with the goal of significantly improving operational model TC position and intensity forecast errors by at least $20 \%$ and $50 \%$ within 5 and 10 years of its initiation, respectively (Gall et al. 2013). By testing and implementing updated models and parameterizations and using data assimilation in various capacities (i.e., Aksoy et al. 2012), position (intensity) forecasts have improved by as much as $60 \%$ $(30 \%)$ in some models. These improvements are not consistent at every forecast time, however, and limitations of representing rapid intensification (Pu et al. 2009) or ERCs (Zhu et al. 2015) still pose a significant problem for the TC modeling and data assimilation communities. 


\subsection{Expectations and Considerations}

Taking the forecasting information into consideration motivates the need to obtain a better grasp on processes that occur in nature. Understanding the physical processes associated with changes in TC surface wind structure can in turn be used to evaluate their appearance in numerically simulated TCs. Similarly derived quantities from model and observational sources can also be used as an alternative method for TC intensity verification purposes (i.e., Vukicevic et al. 2014). Note that some of these ideas have been investigated with aircraft data in a general sense (Uhlhorn et al. 2014), but because of some of the aircraft constraints mentioned above, it is worthwhile to investigate the usefulness of scatterometer that more regularly observe TCs compared to reconnaissance aircraft. With these ideas in mind and in accordance with the plan provided in the dissertation proposal, the expectations of this study are as follows:

- Adequately provide evidence that scatterometer surface wind data are capable of producing reasonable structure analyses through verification against trusted analyses using aircraft SFMR data.

- Distinguish and explain differences in TC surface wind structure in response to vertical wind shear and TC motion; evaluate these differences on global and basinspecific scales, on storm intensity scales, and on storm intensity change scales.

- Evaluate the model intensity verification skill for the scatterometer analysis method relative to the current metric and discuss its usefulness for conditions in which aircraft are unavailable.

- Describe initial analyses comparing the asymmetric surface wind structure to representations of precipitation and convection; provide preliminary explanation of 
the connection between the two parameters and verify results against similar model derived products

Please consider as well that the remaining content chapters are designed for submission to peer-review literature. As such, they are each self-contained with several modifications in their respective introduction and data sections to prevent repetition as much as possible and for cohesion with this document. The results in Chapter 2 are currently published in Geophysical Research Letters (GRL, see references section). The results in Chapters 3 and 4 are currently in review, and Chapter 5 will be submitted later in 2017. 


\section{GLOBAL COMPOSITES OF SURFACE WIND SPEEDS IN TROPICAL}

\section{CYCLONES BASED ON A 12-YEAR SCATTEROMETER DATABASE}

\subsection{Abstract}

A 12-year global database of rain-corrected satellite scatterometer surface winds for tropical cyclones (TCs) is used to produce composites of TC surface wind speed distributions relative to vertical wind shear and storm motion directions in each TC-prone basin and various TC intensity stages. These composites corroborate ideas presented in earlier studies, where maxima are located right of motion in the earth-relative framework. The entire TC surface wind asymmetry is down-motion-left for all basins and for lower strength TCs after removing the motion vector. Relative to the shear direction, the motion-removed composites indicate that the surface wind asymmetry is located downshear-left for the outer region of all TCs, but for the inner-core region it varies from leftof-shear to down-shear-right for different basin and TC intensities groups. Quantification of the surface wind asymmetric structure in further stratifications is a necessary next step for this scatterometer dataset.

\subsection{Introduction}

Understanding of the TC surface wind field, especially within the inner-core, has improved over the past several decades due to observations from TC-penetrating aircraft (Aberson et al. 2006; Rogers et al. 2013) with instrumentation such as the stepped frequency microwave radiometer (SFMR, Uhlhorn et al. 2007; Klotz and Uhlhorn 2014) and Global Positioning System (GPS) dropsondes (Hock and Franklin 1999; Franklin et al. 2003). Contributions from satellite-based instruments, such as QuikSCAT (Draper and Long 2002; Draper and Long 2004; Hoffman and Leidner 2005) and ASCAT (Figa- 
Saldaña et al. 2002) have enhanced the understanding of surface winds in TCs, especially of extended range features

While there is a clear necessity for obtaining surface wind observations in TCs, aircraft and satellite platforms both have their limitations, which makes it sometimes difficult to perform climatological studies. Conversely to the numerous precipitationrelated composite studies (Frank and Ritchie 1999; Corbosiero and Molinari 2003; Lonfat et al. 2004; Chen et al. 2006; Cecil 2007; Jiang 2012; Jiang et al. 2013; Zagrodnik and Jiang 2014; Tao and Jiang 2015), few studies have examined the surface winds in a composite form, mainly due to fewer observations of surface winds in TCs. Several recent studies have used scatterometer data to evaluate storm size and the causes of variability (Chavas and Emanuel 2010; Chan and Chan 2012; Chan and Chan 2015; Chavas et al. 2016), but these articles focus on the outer-core of the TC circulation (generally between $150-300 \mathrm{~km}$ from the TC center). Studies that have provided some examination of the inner-core in a composite sense (Ueno and Kunii 2009, Ueno and Bessho 2011, Uhlhorn et al. 2014, now referred to as U14) are either limited by the amount of data used, data quality, or region of interest. Because of these constraints, it is difficult to diagnose how the inner-core and outer region of the TC surface wind field changes when stratified by TC intensity, storm motion, or vertical wind shear.

In light of the lack of understanding, a 12-year, global dataset of rain-corrected scatterometer surface wind speeds is utilized in an effort to provide a basin-dependent, global climatology of surface wind speeds in TCs. Section 2.3 summarizes the data and methodology used, and Section 2.4 shows the initial results of composite wind speed fields compared to theoretical results with a focus on each TC-prone basin. Section 2.5 
presents the shear- and motion-relative change in wind field structure as a function of TC intensity, and Section 5 presents the conclusions and next steps.

\subsection{Data and Methodology}

Aircraft-based platforms are useful for observing hurricane force wind speeds, but they are unable to provide a snapshot of the full TC wind field. The satellite-based scatterometer is conversely but advantageously equipped to observe a two-dimensional TC surface wind field at a particular time with somewhat reduced horizontal resolution [12.5 $\mathrm{km}$ or $\sim \frac{1}{8} \circ$, (Brennan et al. 2009)]. The data used herein are obtained from QuikSCAT and OSCAT (OceanSat-2, Gohil et al. 2013) scatterometers, which are available through NASA's Jet Propulsion Laboratory (JPL) Tropical Cyclone Information System archive (TCIS, Hristova-Veleva et al. 2013). Scatterometer overpasses are provided for individual cases within each TC basin between years 2000 2011. Abbreviations for each basin or their combinations are used throughout the text (and later chapters) and include: North Atlantic (NATL), Eastern North Pacific and Central Pacific (EPCP), Northwest Pacific (WPAC), and Southern Indian and Southwest Pacific (SHEM). Because scatterometer swaths may miss portions of a TC, a weighting factor between 0 and 1 is calculated from a combination of percentage of TC coverage in the swath within $1.25^{\circ}(\sim 125 \mathrm{~km})$ and $2.5^{\circ}(\sim 250 \mathrm{~km})$ of the storm center. To reduce anomalous results, only cases with weights $>0.7$ are used herein. Over $75 \%$ of the cases have a weight $>0.9$, which indicates that $\mathrm{TC}$ coverage will not hinder or provide additional uncertainties to the wind speed analysis. 
Rain contamination of wind speed and direction necessitates applying a Neural Network (NN) correction to the scatterometer winds (Stiles et al. 2014). Uncertainty in the directional ambiguities remains after the correction, however. This directional issue is being addressed in a current study (Foster et al. 2016) that utilizes an inflow angle model (Zhang and Uhlhorn 2012) to provide a basis for correcting the wind direction. Often these ambiguity issues are not widespread across a swath but tend to be more localized (Stiles et al. 2014; Jun Zhang, personal communication). The applied NN correction allows for accurate assessments of wind speeds regardless of storm strength (with slightly higher uncertainty at wind speeds $>55-60 \mathrm{~m} \mathrm{~s}^{-1}$ ). Figure 2.1 shows an example of wind speeds from Hurricane Katrina on 28 August, 2005. The uncorrected and NN wind fields are provided in the left and right panels, respectively. Clearly, the maximum NN wind speeds are closer to the magnitude of the official maximum wind speed $\left(150 \mathrm{kt}\right.$ or $75 \mathrm{~m} \mathrm{~s}^{-}$ ${ }^{1}$, Knabb et al. 2011) than those of the uncorrected winds.
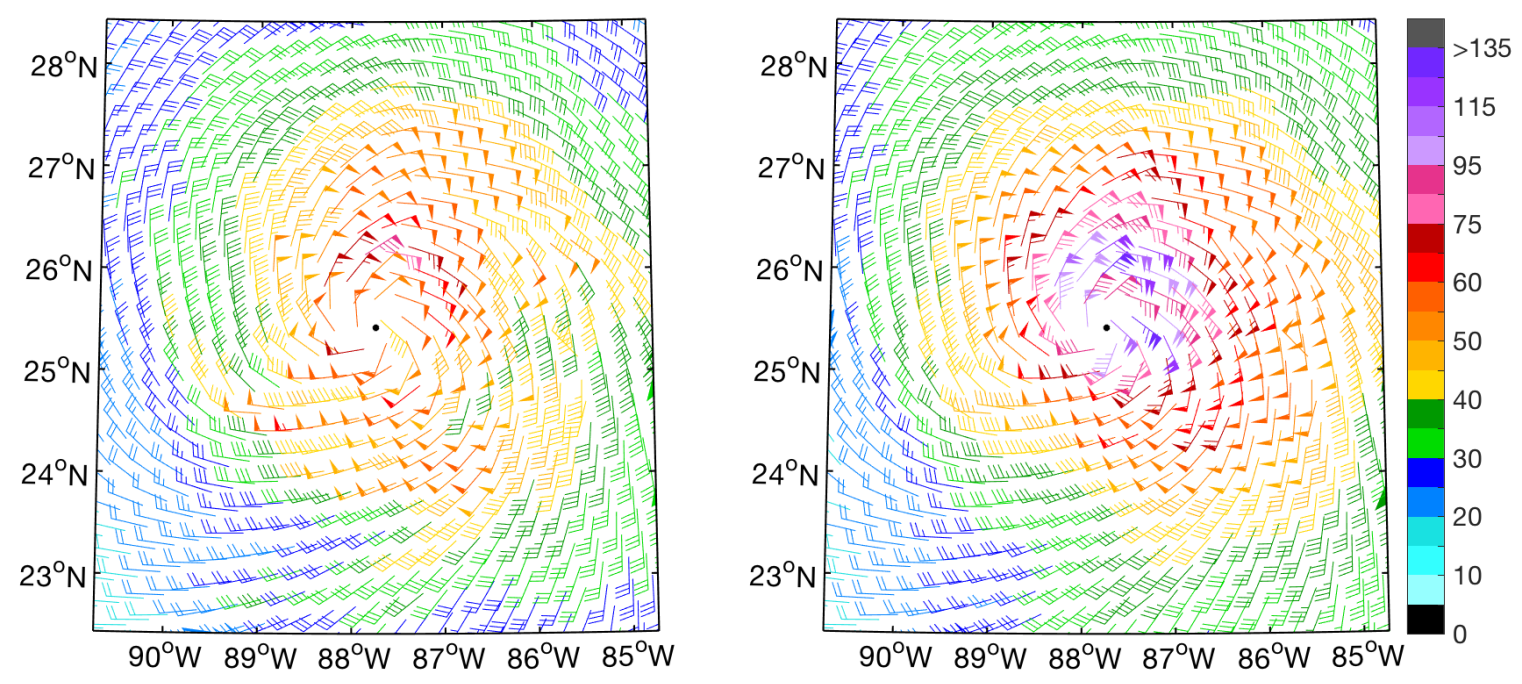

Figure 2.1. QuikSCAT scatterometer surface wind vectors are shown for Hurricane Katrina on 28 August, 2005. The left panel provides the uncorrected wind speeds and the right panel displays the neural network corrected wind speeds (kt, color scale, where $1 \mathrm{kt}$ $\left.\approx 0.5 \mathrm{~m} \mathrm{~s}^{-1}\right)$. Data points are thinned by a factor of two to more clearly show the wind circulation. The black dot indicates the center position. 
For composite analyses, defining a storm center is critical. Unfortunately, the center cannot be defined strictly by the wind circulation because of the direction ambiguity issues previously described, but the backscatter coefficient $\left(\sigma_{0}\right)$ can assist in determining a TC center. In many cases, especially hurricanes, there is a weakness in the $\sigma_{0}$ field near the center due to the presence of a precipitation-free eye. The center for the scatterometer analysis is defined by a combination of circulation, $\sigma_{0}$ (fore and aft horizontal polarization), and interpolated best track position. If the different sources disagree significantly on the center location, best track and $\sigma_{0}$ are given more weight in manually determining the center because of the increased uncertainty in the wind direction. An example of the backscatter coefficient from Hurricane Isabel on 10 September, 2003 with center position indicated is provided in Figure 2.2. For this particular case, the circulation and $\sigma_{0}$ fields coincide well with each other, making it easier to determine the center (black marker in Figure 2.2). In some weaker cases, this is not so easily determined (see Figure 12 in Stiles et al. 2014).

Once a center is determined, the data are converted from an Earth-relative Cartesian grid to a storm-centered polar coordinate grid. While the swath data maintain a $12.5 \mathrm{~km}$ resolution prior to conversion, the radial change in distance between points is not equidistant on the polar grid. Therefore, varying radii along each azimuth could pose a problem for obtaining a reasonable low wavenumber analysis. During the grid conversion, radial errors are calculated, resulting in a mean absolute error of $2.5 \mathrm{~km}$. Incorporating this variability in the radial designation allows for $5 \mathrm{~km}$ windows centered on the desired radius. For example, the lowest radial bin of $6.25 \mathrm{~km}$ includes converted radii between $3.75 \mathrm{~km}$ and $8.75 \mathrm{~km}$. 


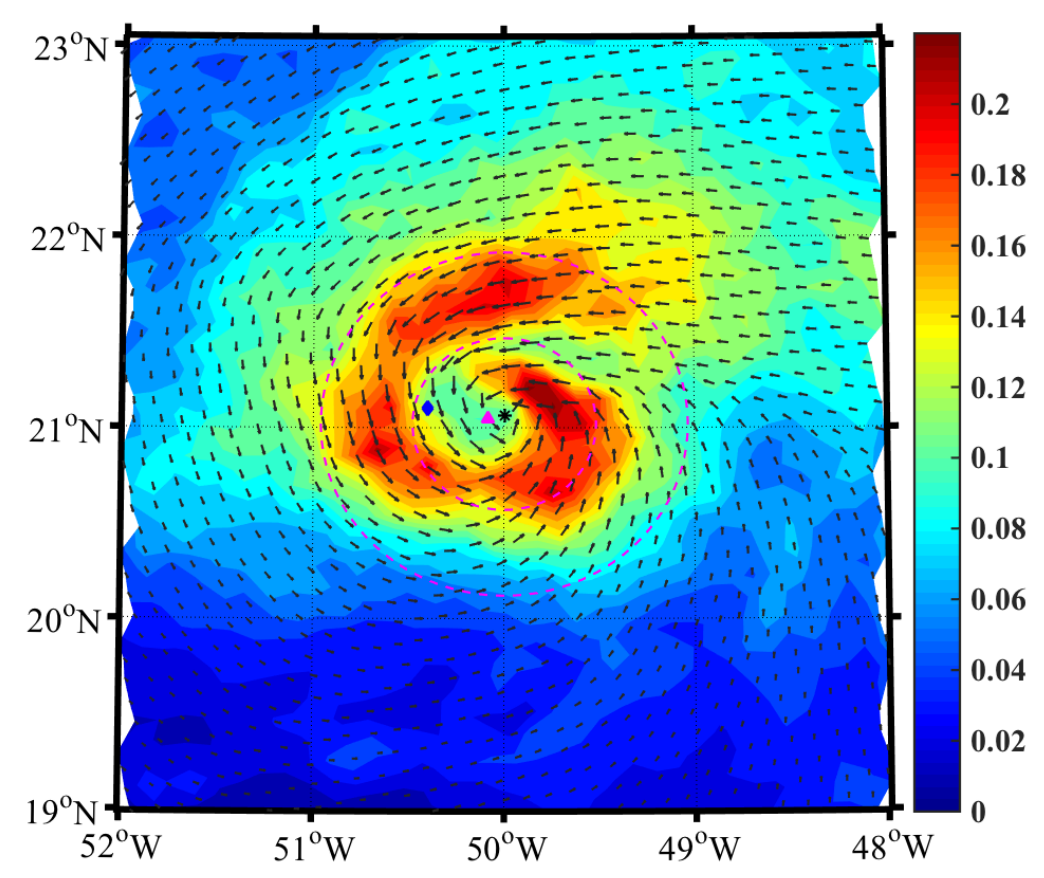

Figure 2.2. Fore and aft (horizontal polarization) combined backscatter coefficient (dB, shaded) and wind vectors are shown for Hurricane Isabel on 10 September, 2003. The black marker within the backscatter weakness and circulation-defined center indicates the center position used in the subsequent low wavenumber analysis. The magenta and blue markers indicate the center positions based on the minimum in backscatter coefficient and best track, respectively.

For a mature hurricane, the inner-core generally extends $\sim 200 \mathrm{~km}$ from the storm center (i.e., Uhlhorn and Nolan 2012, U14). For the current study, which includes weaker tropical storms and hurricanes, it is necessary to extend this distance by $50 \mathrm{~km}$ to ensure the processes associated with the inner-core are captured for most storm sizes. In fact, Chavas et al. (2016) notes that analysis beyond $\sim 250-300 \mathrm{~km}$ is not meaningful for this type of study. Based on this definition for TC inner-core, the radial binning process extends to $250 \mathrm{~km}$ from the storm center with increments of $6.25 \mathrm{~km}$.

\subsubsection{Determination of a Radius of Maximum Wind from Scatterometer Data}

As part of the analysis procedures, it is necessary to calculate a radius of maximum wind (RMW) with each scatterometer case. The RMW is calculated as an 
azimuthal average of the maximum wind speeds in all storm-relative quadrants. For reference, a standard reconnaissance flight has radial legs on the order of 105 nautical miles (194 km), which is designed to capture the TC circulation (Uhlhorn and Nolan, 2012; U14). Based on airborne Doppler radar data from the NOAA WP-3D, the average RMW (at $2 \mathrm{~km}$ altitude) is $32.6 \mathrm{~km}$ on average (Rogers et al. 2012). Using their average eyewall tilt of $28^{\circ}$, the average surface RMW is on the order of $29 \mathrm{~km}$. This result is supported by the SFMR cases used in U14, which estimates a mean surface RMW of $\sim 25$ $\mathrm{km}$. The cases used in U14, however, were all mature hurricanes with well-defined circulations.

For the scatterometer dataset, all TC intensities are used, and weaker storms tend to have much larger RMW as their circulations are fairly disorganized. Ueno and Bessho (2011) note that the resolution of their scatterometer data limits any observations within $50 \mathrm{~km}$, and they bound their RMW between 50 and $150 \mathrm{~km}$. Their average RMW was 99 $\mathrm{km}$. Keep in mind that TCs over Northwest Pacific tend to be larger than hurricanes found over the North Atlantic (Chavas and Emmanuel 2010; Chan and Chan 2012; Chavas et al. 2016). Because the resolution of the data used in the present study is increased two-fold from Ueno and Bessho (2011), it is possible to obtain a minimum RMW near $25 \mathrm{~km}$. Because a global dataset is used, it is important that all attainable storm sizes are included, with the exception of very large storms (RMW $>125 \mathrm{~km}$ ). By providing an upper RMW bound, it ensures that all cases can be examined to at least $2 \times \mathrm{RMW}$, and that the inner core of the $\mathrm{TC}$ is provided.

Basin statistics regarding mean and standard deviation of RMW as well as other parameters in the dataset are described in Table A1 in Appendix 1. Table 2.1 shows 
detailed RMW statistics for each TC basin as determined by the scatterometer data. Figure 2.3 shows the difference in distribution of RMW for each TC basin. Probability distribution functions (PDFs) indicate that while there are some differences in size between each basin, the scatterometer determines a $\sim 30-35 \mathrm{~km}$ RMW peak probability for all basins. Interestingly, the shape of the curve of the NATL scatterometer data is similarly shaped to that of the SFMR for North Atlantic cases, which confirms that the RMW from scatterometers differ mainly due to their coarser resolution. Please note that Figures 2.1-2.3 and Table 2.1 are not published in Klotz and Jiang (2016) due to length limitations of the journal. Although submitted with Klotz and Jiang (2017), these items are included in this chapter for improved flow of the full dissertation.

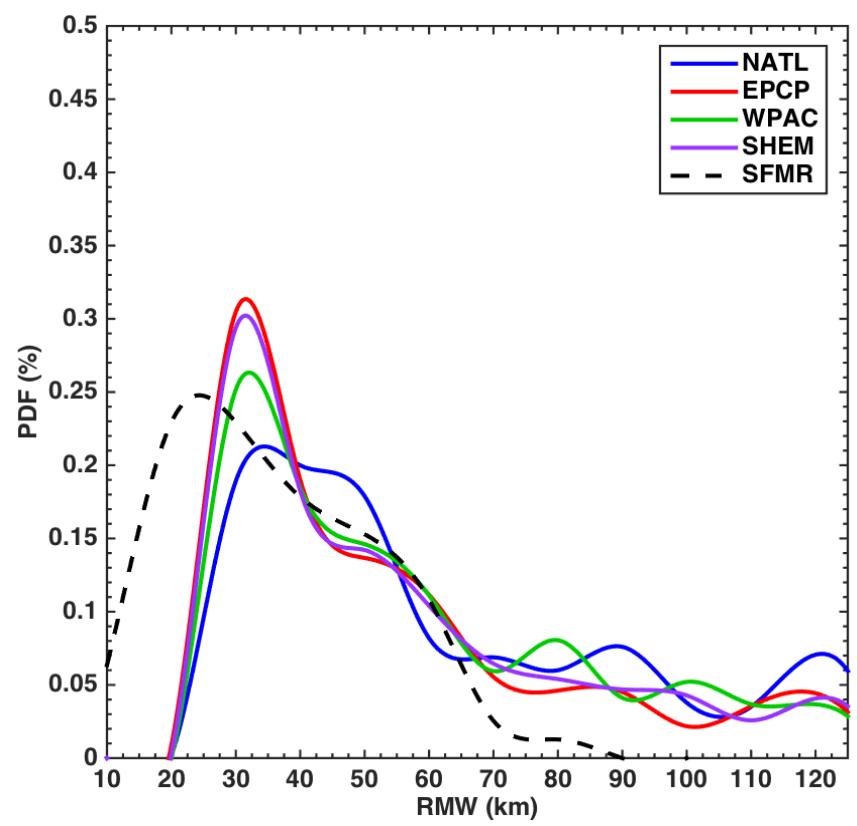

Figure 2.3. Probability density functions (PDFs) of RMW for all cases are provided, with North Atlantic (NATL, blue), Eastern and Central North Pacific (EPCP, red), Northwest Pacific (WPAC, green), and South Indian and Southwest Pacific (SHEM, purple) curves specifically represented. The PDF of RMW determined from SFMR data are also shown for North Atlantic cases (black dashed line). 
Table 2.1. Basic statistics including mean, median, minimum, maximum, and peak percentage of RMW for each examined TC basin. RMW and frequency of occurrence are listed for the peak value. Similar values are provided for RMW determined from SFMR flights over the North Atlantic (U14).

\begin{tabular}{|c|c|c|c|c|c|}
\hline & NATL & EPCP & WPAC & SHEM & SFMR \\
\hline Mean $(\mathrm{km})$ & 64.2 & 57.2 & 61.8 & 59.4 & 35.9 \\
\hline Median $(\mathrm{km})$ & 55.3 & 47.8 & 54.1 & 51.3 & 34.6 \\
\hline Minimum $(\mathrm{km})$ & 27.4 & 28.5 & 30.9 & 28.7 & 9.9 \\
\hline Maximum $(\mathrm{km})$ & 125.0 & 125.0 & 125.0 & 125.0 & 81.5 \\
\hline Peak Frequency $(\mathrm{km},[\%])$ & $34.2[21.7]$ & $31.6[32.1]$ & $32.0[27.3]$ & $31.6[29.8]$ & $24.3[24.8]$ \\
\hline
\end{tabular}

\subsubsection{Scatterometer Verification and Other Data Considerations}

Because of the lower horizontal resolution of the scatterometer compared to aircraft observations from SFMR (Uhlhorn et al. 2007, Klotz and Uhlhorn 2014), it might be assumed that scatterometer data are limited in their ability to provide assessments of TC surface structures. This question stems from the notion that the scatterometer cannot resolve an accurate RMW and provide valid radial profiles. While the RMW is generally 10-15 km larger than determined from SFMR (Figure 2.3 and Table 2.1), scatterometer wind speeds (with NN correction) compare reasonably well to those from the SFMR. Figure 2.4 provides a scatterplot and weighted linear regression fit for the maximum wind speed observed from the SFMR and from the scatterometer. Coincident times are determined as \pm 5 hours of the initial SFMR time. Although the root mean squared error (RMSE) is higher than desired, it is clear that scatterometers can produce maximum wind values on par with operational aircraft data. It should also be noted that when comparing cases that have larger RMW (the lower limit of the scatterometer is $\sim 25 \mathrm{~km}$ ), the fit is less variable and the RMSE drops to $\sim 4.5 \mathrm{~m} \mathrm{~s}^{-1}$. Therefore, larger storms will provide a better opportunity to observe the maximum surface wind. 


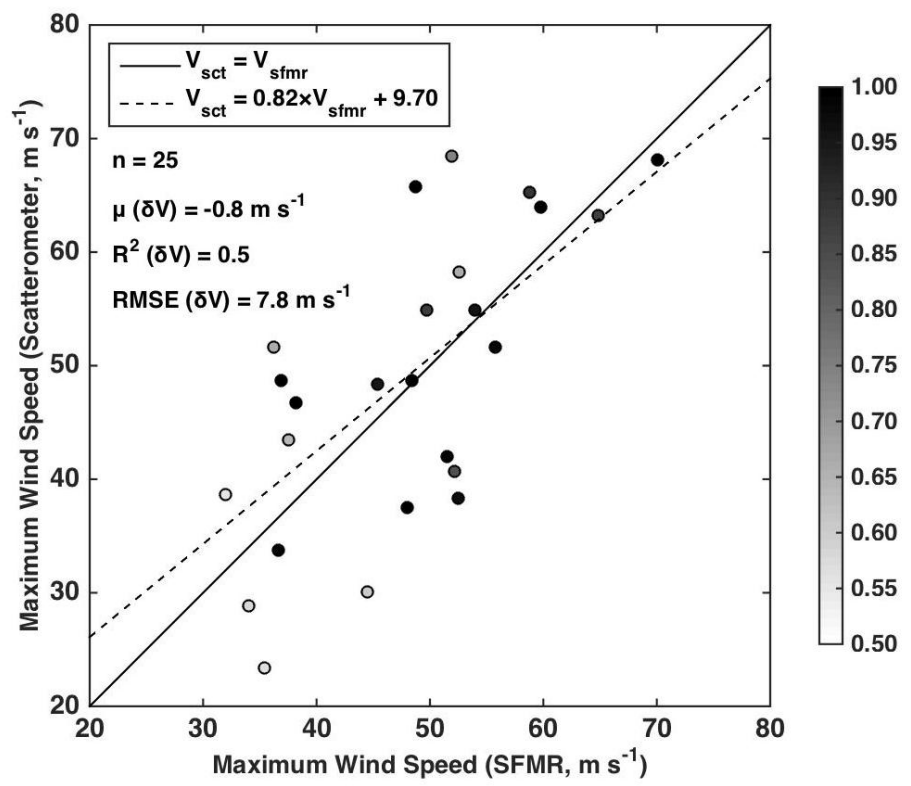

Figure 2.4. Markers indicate the coincident pairs ( \pm 5 hours) of maximum surface wind speed $\left(\mathrm{m} \mathrm{s}^{-1}\right)$ from SFMR and scatterometer. Shading of the markers indicates the weight applied to the scatterometer swath. A weighted, linear regression fit (dashed line) is compared to the perfect fit (solid line), with the fit equation provided in the legend. The symbol $\delta \mathrm{V}$ indicates that the printed quantity is based on the difference between the paired maximum wind speeds.

To present the wind fields in composite form, the scatterometer winds are placed on a polar grid with radius normalized by RMW. One might question normalizing the radial grid by the RMW, but in order to truly understand the inner-core structure, normalizing allows for a better evaluation in the composite framework than if using a standard radial grid. Figure 2.5 provides supporting evidence that an ample number of cases exist at extended radii to have a reasonable representation of the wind field in the RMW framework. 

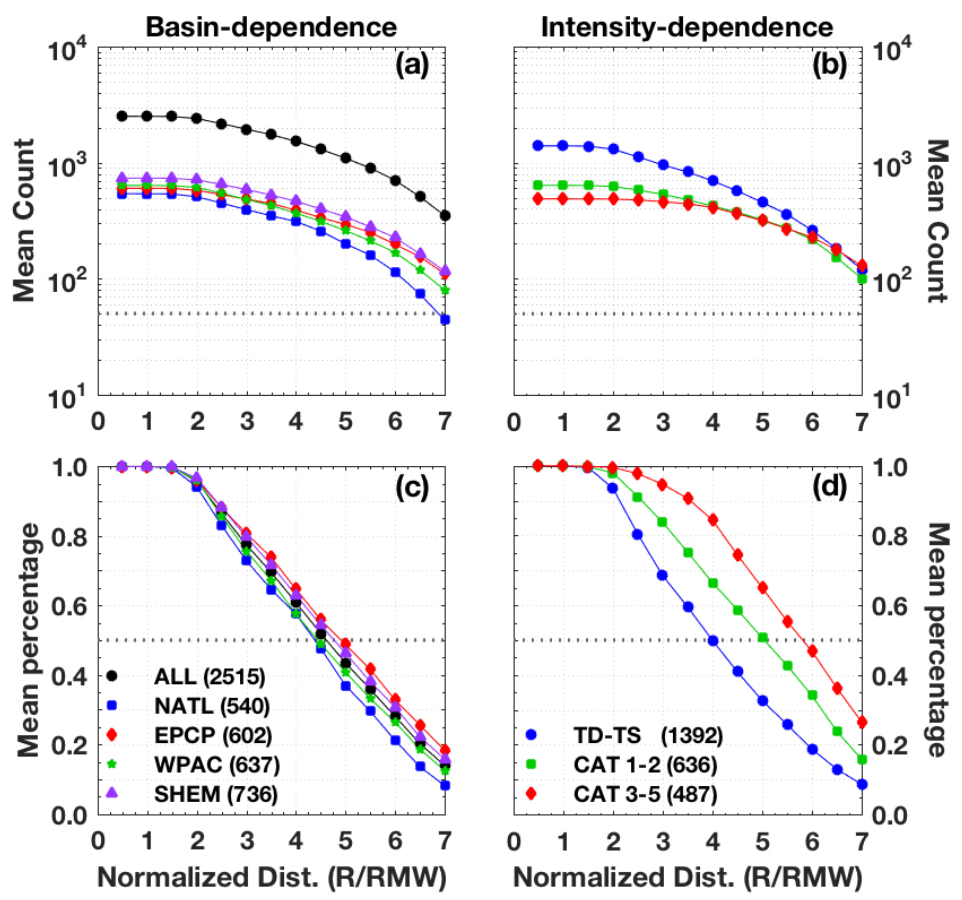

Figure 2.5. Azimuthal mean normalized radial profiles of the number of cases (a-b) and the percentage of cases (c-d) for various stratifications within the scatterometer dataset. Panels $(a, c)$ indicate profiles for all cases and for the specific basins. Panels $(b, d)$ show values for TC intensity. In the top two panels, which are plotted on a logarithmic scale, the dashed gray line indicates the minimum allowable number of cases (50), while the same line in the lower panels indicates $50 \%$ of samples for the respective profile. The total number of cases for each profile is included in the legend.

In Figure 2.5(a, b), the number of cases never drops below 50, which is deemed as the minimum number for obtaining a reasonable analysis (Tao and Jiang 2015). In panels (c,d), the percentages drop below 50\% at radii $>4-5$ RMW in many composites. While the percentages of the total cases decline steadily after this radial limit, the number of cases is large enough to expect reasonable results within the full two-dimensional domain.

The best track locations and official storm characteristics are obtained from HURDAT2 (Jarvinen et al. 1984; Landsea and Franklin 2013) and Joint Typhoon Warning Center (JTWC) reports. Generally, the wind direction provides an adequate 
center estimate (see note above related to directional ambiguity), but the backscatter coefficient provides verification of the wind-derived center. For reference, Table A1 lists the number of scatterometer passes for each basin based on TC intensity as well as some other key storm and environmental characteristics. Two-dimensional fields are then rotated based on the motion heading from the best track sources mentioned previously or for shear heading provided in the Statistical Hurricane Intensity Prediction Scheme (SHIPS) database (DeMaria and Kaplan 1994; DeMaria and Kaplan 1999).

Shear is calculated for a deep layer (between 850 and $200 \mathrm{hPa}$ ), and the vortex has been removed through $500 \mathrm{~km}$ radially outward of the TC center. One might argue the representativeness of the deep-layer shear for shallow TCs. A shear profile would provide a better estimate of the shear at different levels (Elsberry and Jeffries 1996), but several studies have shown that strong deep-layer shear negatively impacts the development of weak TCs (Frank and Ritchie 2001, Heymsfield et al. 2006). Evaluation of the shallowlayer shear vector in SHIPS, which arguably still contains impacts from a vortex, provides inconclusive results. For weaker TCs examined by basin, NATL shallow shear vectors differ from the deep-layer shear more in speed than heading, where median differences are $5 \mathrm{~m} \mathrm{~s}^{-1}$ and $-8.0^{\circ}$, respectively. The opposite is true for WPAC cases, where median shear headings differ by $-28.5^{\circ}$ but shear speeds differ by $2.5 \mathrm{~m} \mathrm{~s}^{-1}$. This comparison suggests there is not enough evidence to indicate that a shallow shear is more representative than the deep-layer shear in terms of affecting the shallow vortex. Therefore, the deep-layer shear should be an appropriate metric for evaluating the wind shear for tropical depressions and storms. PDFs of deep-layer shear and motion direction 
associated with the scatterometer cases for each basin are displayed in Figure 2.6(a) and (c), respectively.
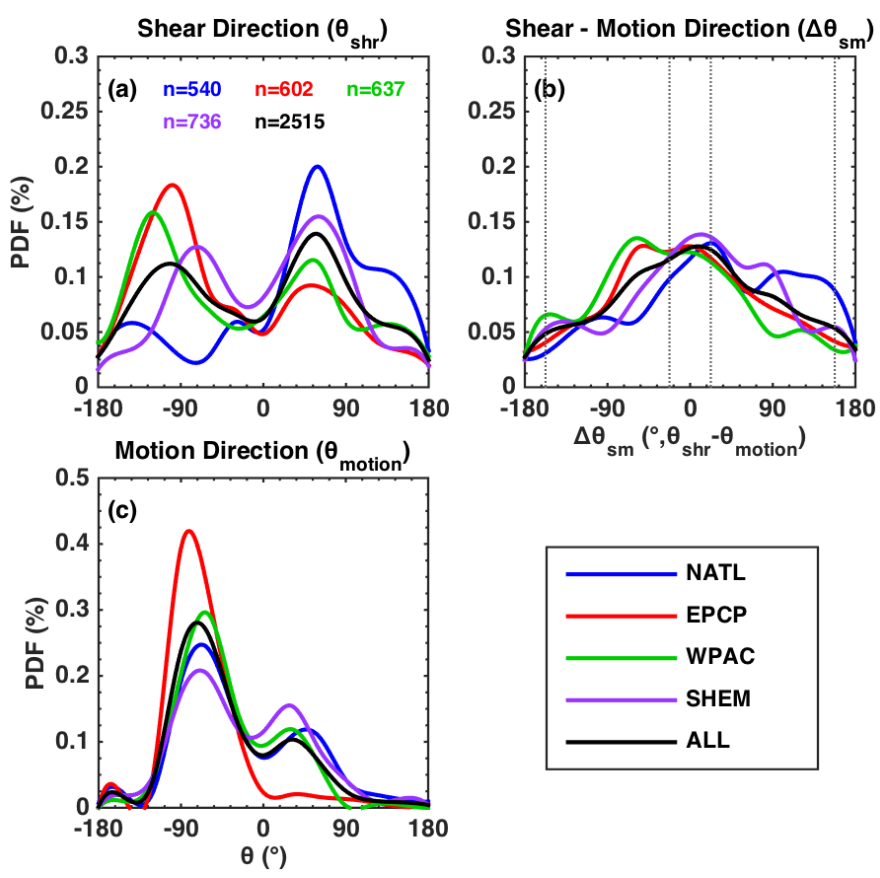

Figure 2.6. Probability density functions of (a) Shear direction $\left(\theta_{\text {shr }},{ }^{\circ}\right)$, (c) Storm motion direction $\left(\theta_{\text {motion }},{ }^{\circ}\right)$, and (b) Shear - Motion direction $\left(\theta_{\text {shr }}-\theta_{\text {motion }},{ }^{\circ}\right)$, for each individual basin as well as for all basins combined are displayed. The dashed vertical lines in (b) are the angle difference thresholds as discussed in Chen et al. (2006).

All basins have similar storm motion distribution, but not all basins experience the same shear preferences. Where the WPAC and EPCP favor an easterly (i.e., $-90^{\circ}$ ) to northeasterly shear, the NATL and SHEM favor a southwesterly to westerly shear (SHEM variables were rotated to northern hemisphere reference frame). Wind shear has large variability as indicated by the somewhat bimodal signal in the PDFs, but it is also important to examine shear's relationship to motion, displayed as a shear and motion angle difference in Figure 2.6(b). Designating whether the shear is to the right or left of motion is based on the information provided in Chen et al. (2006). 
2.4 Comparison to Theoretical and Observational Studies Using Basin-dependent Composites

Several theoretical modeling studies (Shapiro 1983 [herein referred to as S83]; Kepert 2001; Thomsen et al. 2015) examined structural properties of TC vortices in regards to boundary layer convergence and upward motion. Despite using different models, they conclude that the maximum total wind speed in the boundary layer is downmotion-right in an earth-relative frame. This is consistent with some observational case studies using in-situ wind measurements (Powell 1982, Figure 9) and aircraft remote sensing data (U14 Figure 8b). S83 also showed a down-motion-left maximum of total wind in a motion-relative frame (after removing the motion vector, his Figure $5 b$ ). Subsequent references to motion-relative quadrants are abbreviated in the form DM for down-motion or UM for up-motion. Addition of ' $\mathrm{R}$ ' or ' $\mathrm{L}$ ' signifies right or left of the motion vector. Similar abbreviations follow for shear-relative quadrants, where ' $\mathrm{S}$ ' is used instead of 'M' (i.e. DS instead of DM).

Using the scatterometer data, it is possible to test these modeling or observational case-based results within each basin and as a function of storm motion and wind shear in a statistical, composite way. In all composites, storm-centered wind speeds are normalized by the maximum of the composite to more easily compare between the various stratifications. In an earth-relative reference frame (motion vector not removed), shown in the top row of Figure 2.7 all basins display a nearly $90^{\circ}$ right-of-motion orientation, with a little less than $90^{\circ}$ for EPCP storms. Note that SHEM cases have been rotated to a Northern Hemisphere grid by mirroring around the motion or shear direction to account for the Coriolis effect as in Chen et al. (2006). 

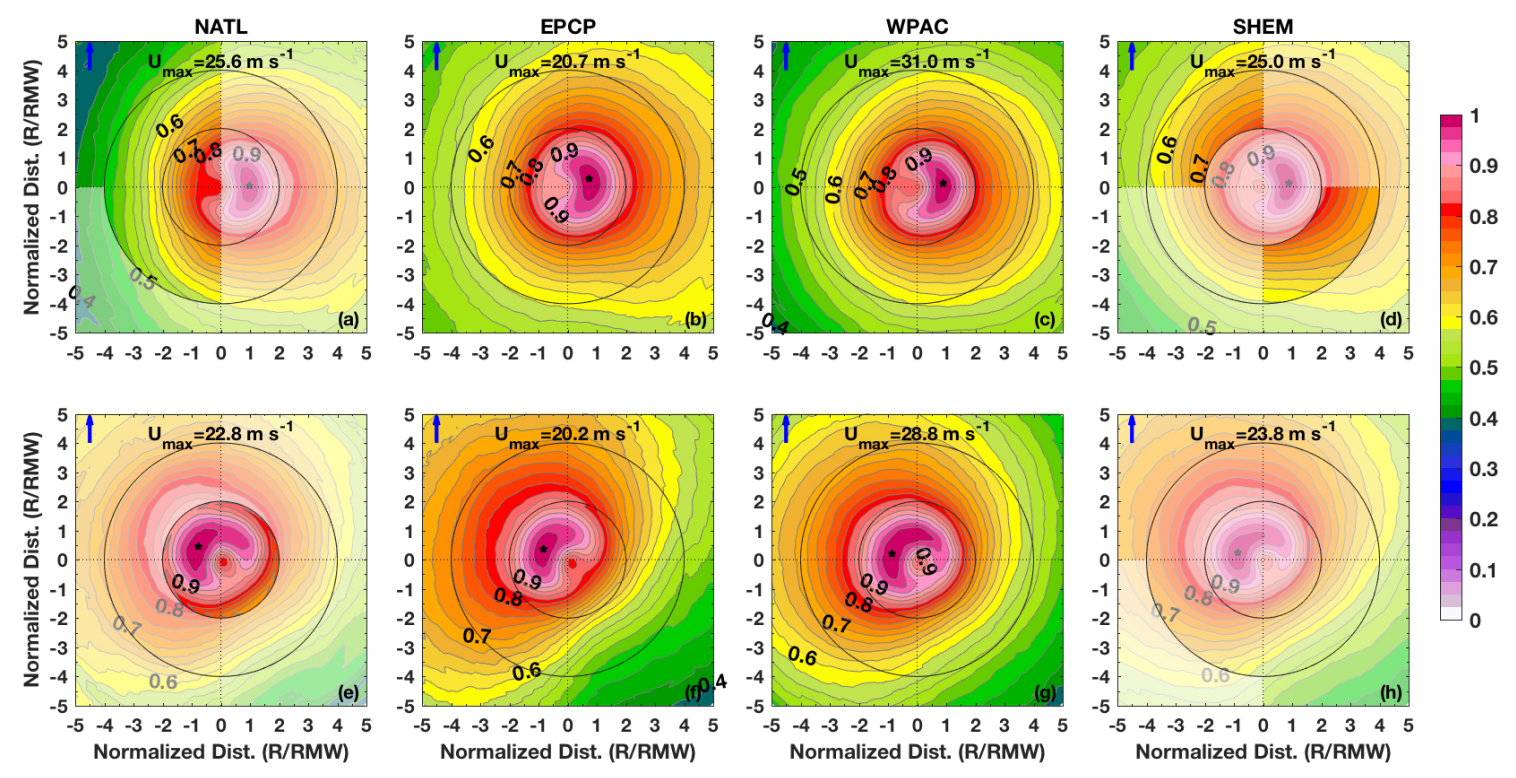

Figure 2.7. Composite, normalized wind speed analyses are provided for NATL, EPCP, WPAC, and SHEM in a motion-relative reference frame with and without translation effects in (a-d) and (e-h), respectively. The storm-centered figures are plotted on a radial polar grid using a normalized radius (R/RMW). Contour lines are plotted in increments of 0.025 normalized units, where a value of 1 is equal to the maximum. The blue arrow is the direction of the motion vector and the black marker indicates the location of the maximum wind speed. The maximum wind speed value is also indicated on each panel for reference. Lighter shading indicates areas that do not attain $95 \%$ statistical significance when compared to the global composite.

Statistical significance within each composite analysis is computed using a paired Kolmogorov-Smirnov test at each grid-point, determining the median p-value, and using this p-value as an overall estimate of each motion- (or shear-) relative quadrant. Because analysis at extended radii $(>4-5 \times \mathrm{RMW})$ in this framework is considered less meaningful (Chavas et al., 2016), composite results are only presented to $5 \times$ RMW. Lighter shading in the NATL and SHEM composites indicate regions where statistical significance (95\%) is not attained. The results convey that the global composite (not shown here) most closely resembles these two basin composites and that EPCP and WPAC composites deviate significantly from the global composite. The detailed significance values for 
Figure 2.7 as well as for other composites presented are provided in Tables A2 and A3 in Appendix 1. Although the orientation displayed differs from the DMR preference found by the previous studies mentioned above, it is consistent with rather earlier results (Rossby 1948; Kuo 1969; George and Gray 1976; Jones 1977; Brand et al. 1981; Holland 1983a, 1984; Chan and Williams 1987). Traditionally, earth-relative surface wind fields have been approximated as a simple translating axisymmetric vortex with a wavenumber1 asymmetry maximized RM.

Motion-relative composites (with motion vector removed) are provided in Figure 2.7(e-h). All composites are now oriented DML, further confirming that the translation speed has a significant impact on the asymmetry structure of the wind field. Interpretation of statistical significance is similar to Figure 2.7(a-d). In this framework, S83 indicates that the boundary layer flow is maximized DML, which is consistent with what is found here. Using U14 as an approximate guide, flight level wind maxima are generally $45-60^{\circ}$ upwind of the surface maxima at translation speeds comparable to the mean for the scatterometer composites, although the vortex translation has not been removed in their analysis. The SFMR cases in the validation of the scatterometer winds (Figure 2.4) are used to provide an initial assessment of the conditions with the motion removed. The wavenumber- 1 phase of the maximum surface and flight-level wavenumber- $0+1$ amplitude is calculated and motion is removed following the coefficients in U14's Table 2. Although the sample here is small, the peak flight-level asymmetry approximately occurs near $-30^{\circ}$ (DML) and the surface phase peaks between $-30^{\circ}$ to $-60^{\circ}$ of the flightlevel phase. This result provides support for the result shown in Figure 2.7, where the scatterometer wind maxima are generally $\mathrm{LM}$ to $\mathrm{DML}\left(-90^{\circ}\right.$ to $-60^{\circ}$ of motion direction). 
Figure 2.8 provides a similar analysis but in a shear-relative reference frame. Previous surface wind speed analyses have shown preference for DSL orientations within smaller samples (Ueno and Bessho 2011; U14). As seen in Figure 2.8(a-d), before removing the motion vector, the surface wind asymmetry has a high degree of variability between the basins. In the NATL composite, the shear is generally in the same direction as or to the right of motion (Figure 2.6) and the wind field is mostly oriented DS. This result is consistent with a DMR to RM orientation as in Figure 2.7(a). The WPAC composite experiences shear that is generally to the left or in the same direction as motion. Figure 2.8(c) indicates a DSR maximum wind speed, but the innermost contour is rotated USR, which is also consistent with the significant influence from motion from Figure 2.7(c).
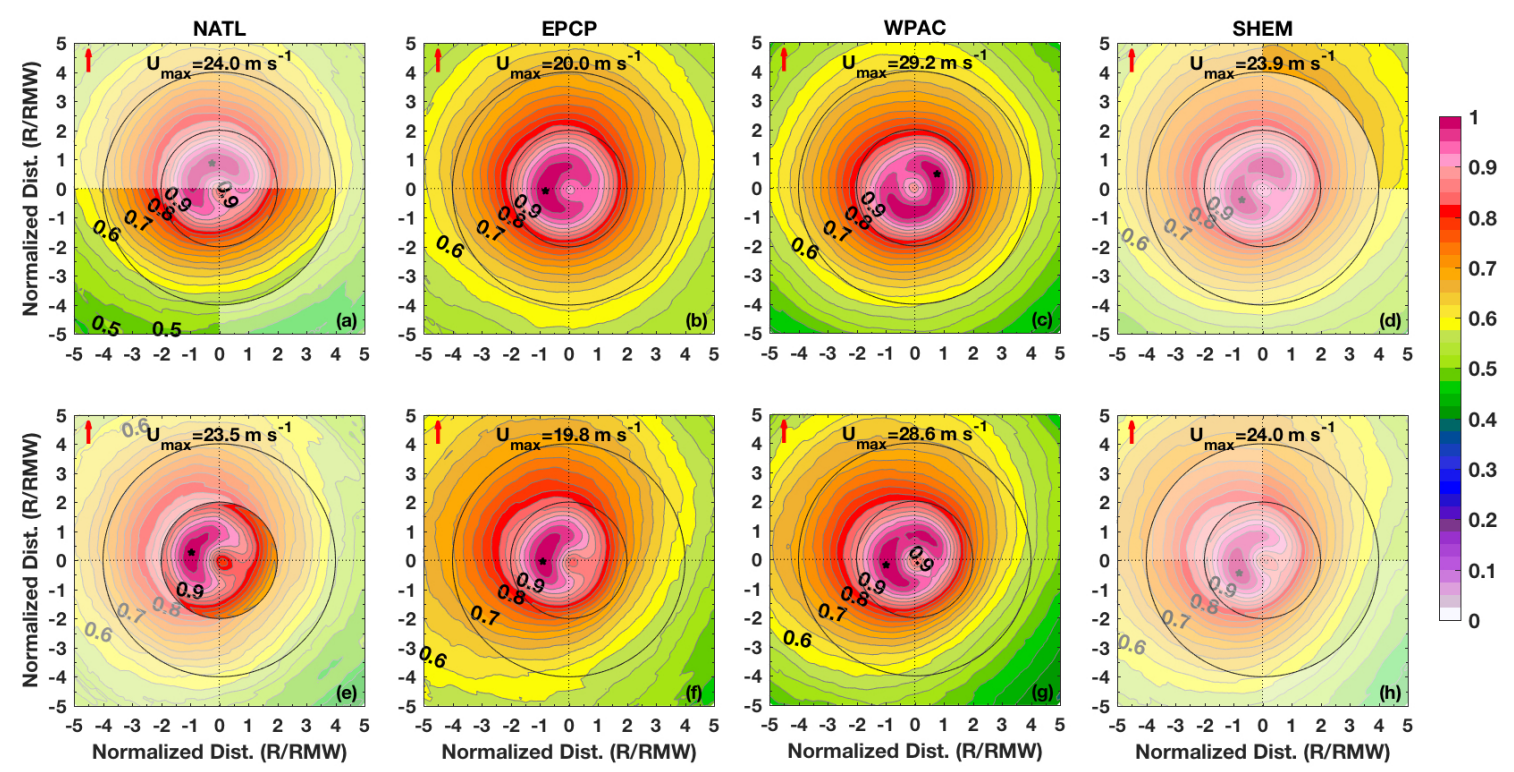

Figure 2.8. Similar to Figure 2.7 but for a shear-relative reference frame. The red arrow indicates the shear direction.

After removing translation effects, all basin composites show a DSL asymmetry for the mid-range $(2-4 \times \mathrm{RMW})$ and outer $(>4 \times \mathrm{RMW})$ region of the surface wind field 
For the inner-region $(<2 \times \mathrm{RMW})$, the asymmetry maximum is mainly LS with some variation either DSL or USL. U14 provides support for this result as well, where they indicate that as shear increases, the surface wavenumber-1 phase rotates downwind from DS to LS. Because the mean shear values for the basin-specific composites range from 6$8 \mathrm{~m} \mathrm{~s}^{-1}$, the results in Figure 2.8 are slightly downwind of U14's location. Most of the panels here are statistically significant at $95 \%$. It is encouraging that the analyses developed from the scatterometer overpasses, despite their lack of horizontal resolution, are capable of producing results that agree with previous studies.

\subsection{Wind Shear and Storm Motion Impact based on TC Intensity}

Improvements upon the results of U14 and Ueno and Bessho (2011) are accomplished by stratifying storms based on their intensity. Their results are specific for hurricane intensities while the global scatterometer database contains a substantially large sample at all TC intensities. Figure 2.9 provides the motion- and shear-relative composites as a function of $\mathrm{TC}$ intensity with similar results to those previously mentioned (RM- and variably-oriented shear-relative fields). The focus for this section is on the same composites but with motion removed. Figure 2.10 provides normalized wind speed composites as a function of intensity for motion- and shear-relative reference frames in panels (a-c) and (d-f), respectively. All panels are statistically significant at $95 \%$ through $4-5 \times$ RMW. 

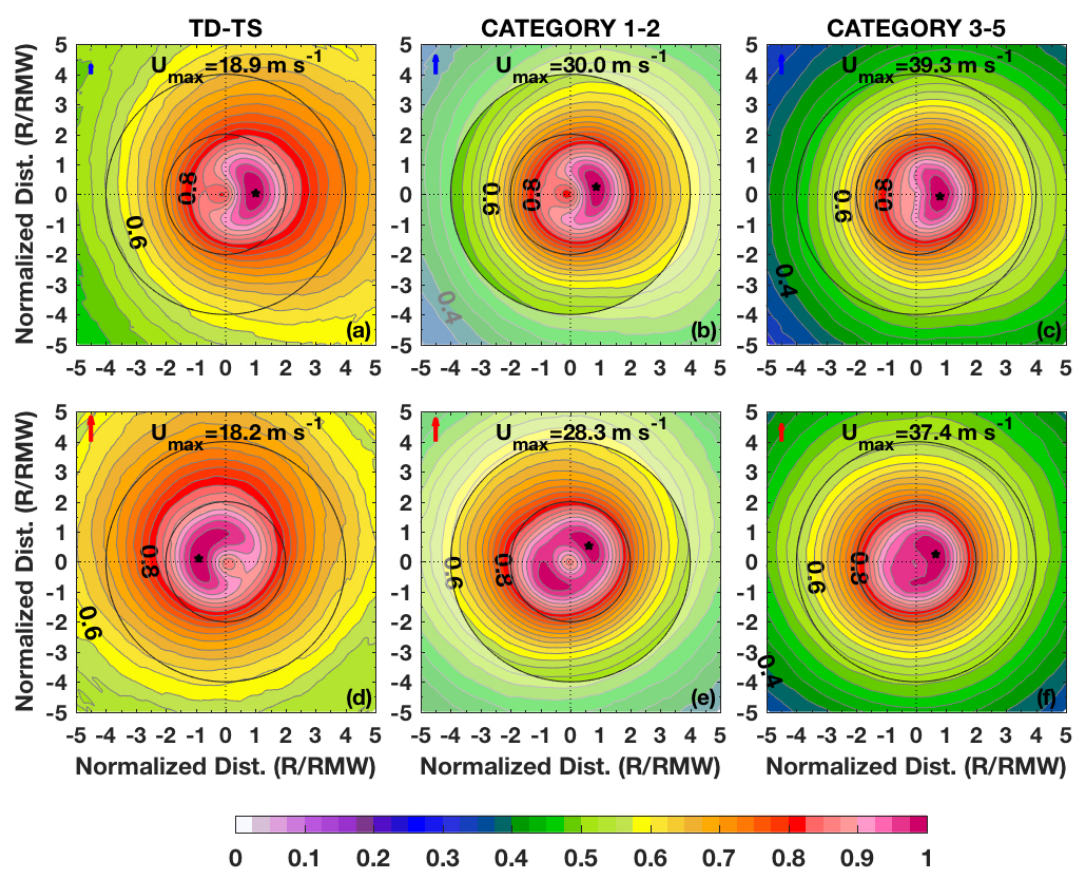

Figure 2.9. Presented similarly to Figure 2.7 but for all basins combined and represented by different TC intensity groups, where $(\mathrm{a}, \mathrm{d})$ are tropical depressions and storms, $(\mathrm{b}, \mathrm{e})$ are non-major hurricanes, and (c,f) are major hurricanes. All panels retain translation effects. The top (bottom) row is shown in a motion (shear) relative reference frame.
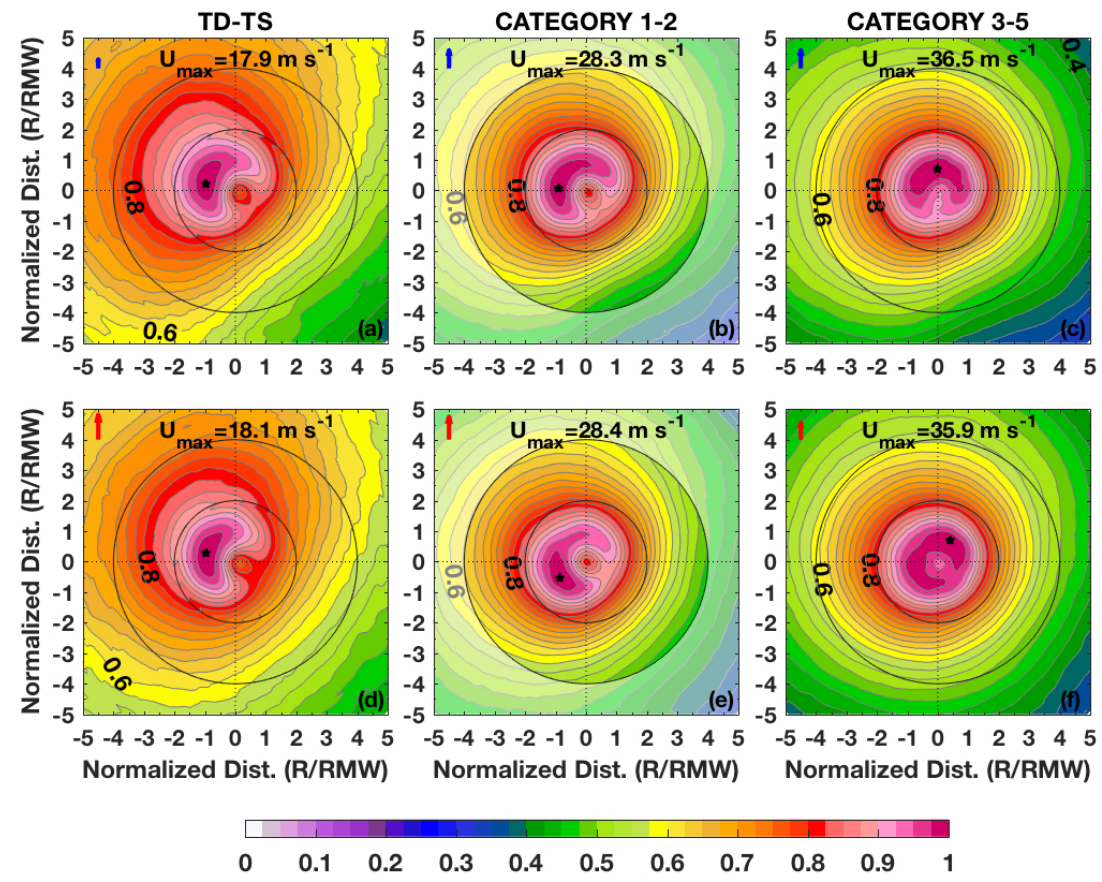

Figure 2.10. Similar to Figure 2.9 but with translation effects removed. 
The mid-to-outer region of all TC intensity groups and the inner region of nonmajor hurricane cases (tropical storms and Category 1-2 hurricanes) in Figure 2.10(a-c) display a surface wind asymmetry oriented DML as with the basin composites. Major hurricanes have a DM oriented maximum in the inner region with rotation DML as radius increases. Due to the presence of moderately strong shear within weak TC's (see Table A1), it is likely that shear is impacting the structure close to the peak winds. As intensity increases and shear decreases, the composites indicate possibly more impact from motion than from shear due to the increased radial extent of the DM signature.

In the shear-relative composites of Figure 2.10, the outer region of all TC intensity groups and the inner region of tropical depressions and tropical storms display a surface wind asymmetry oriented DSL. However, for the inner region, the Category 1-2 hurricane group shows an USL asymmetry, while the major hurricane group shows a DSR asymmetry. This indicates that the shear likely has a large impact on a TC's mid-toouter region for hurricanes, but has impacts on the entire wind field of tropical depressions and storms. On the other hand, even after removing the motion vector, the residual motion influence is still strong enough in major hurricanes to rotate the motion and shear-relative structure upwind of the preferred DML and DSL orientations, respectively. This orientation result speaks to vortex resiliency to shear and motion residual effects as the intensity increases (Reasor and Eastin 2012; Reasor et al. 2013). U14 suggests a 3-5 $\mathrm{m} \mathrm{s}^{-1}$ additional contribution of the asymmetry amplitude from shear, but this can be evaluated when quantifying the asymmetry in the future. Additionally, results that compare the full range of storm motion to slow moving cases (not shown, not statistically significant) indicate that Category 1-2 hurricane orientations rotate 
anticyclonically from DML to DM. Tropical storms and major hurricanes are almost unchanged in their motion-relative structure, which suggests that the residual motion effects for weak systems are substantially lower in strength than the shear influence. For strong hurricanes, the opposite relationship may exist, where residual motion factors significantly influence at least the inner region of the TC. It would then be plausible to consider that Category 1-2 hurricanes represent a transition threshold between which motion and shear impacts are both influencing the vortex in similar capacities. Note that friction velocities in TCs increase with increasing wind speed up to $\sim 40 \mathrm{~m} \mathrm{~s}^{-1}$, where they begin to decrease slightly (Powell 1980; Powell et al. 2003). This knowledge of friction supports the hypothesis that as shear decreases with increasing intensity and friction velocity is maximized near Category 1-2 strength, the primary impact on structure might be alternating from shear to residual motion factors. Also considering that Chan and Chan (2015) determined a threshold latitude at which storm size maximizes, changes with preferred motion and shear heading and speed with increasing latitude likely contribute to some of the variation in the observed surface wind structure as well. For confirmation of the change in shear/motion direction and speed as a function of latitude, Figure 2.11 shows the PDFs of four $10^{\circ}$ latitude bins. Clearly, the presence of easterlies at lower latitudes and westerlies at higher latitudes is exhibited in the shear panels (a-b). The transition of the motion direction appears to be at higher latitudes than for shear. 

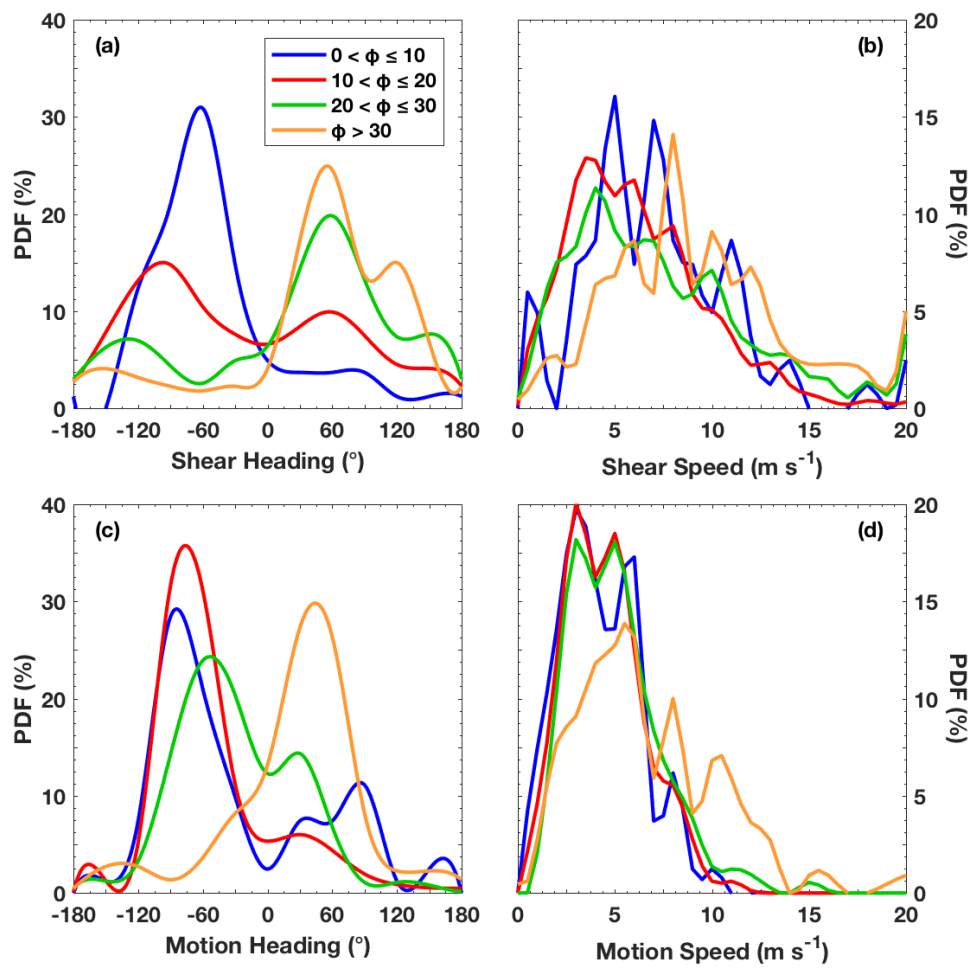

Figure 2.11. Presented as a function of latitude $\left(\phi\right.$, binned every $\left.10^{\circ}\right)$, PDFs of shear heading and speed and motion heading and speed are displayed in panels (a-b) and (c-d), respectively.

\subsection{Conclusions}

TC-centered composite wind speed fields were developed based on a large database of scatterometer surface winds that were able to reproduce results that confirm our current understanding of motion-relative and shear-relative asymmetric structure. In the earth-relative framework before removing the motion vector, the motion impact is dominant over the shear impact, producing a RM asymmetry that is consistent with many earlier studies. The basin-specific composites provide insight into the motion-relative asymmetric field as described in previous theoretical studies by S83, Kepert (2001), and Thomsen et al. (2015) and observational studies by Ueno and Bessho (2011) and U14. After removing the motion vector, the residual motion effects along with shear effects produce a DML asymmetry of surface wind except for the inner region of major 
hurricanes. Based on the earth-relative results, the shear impact on surface wind asymmetry of TCs is secondary to the motion impact, which is the opposite as for the precipitation/convection asymmetry. Many previous studies have shown a sheardominant precipitation asymmetry with a DSL orientation (e. g. Chen et al. 2006). The shear impact on surface wind appears only after removing the motion vector. The motionremoved composites in the shear-relative framework show a DSL surface wind asymmetry for the outer region of all TCs and the inner region of tropical depressions and storms. For the inner region, the shear-relative asymmetry changes in orientation as a function of intensity. Therefore, the authors hypothesize a possible transition intensity range within non-major hurricanes at which motion and shear are similar in their influence on a vortex. Deviations from these structures are possible depending on the shear strength, and shear's relationship to motion plays a crucial role in determining variation of the asymmetric structure. These factors are necessary to consider when quantifying the asymmetric structure.

From the results presented, the next two chapters include assessment and quantification of the low wavenumber asymmetric surface wind structure as it relates to wind shear and storm motion and the difference between their direction and strength. Additionally, intensity change impacts the asymmetric structure in the precipitation/convective components of TCs and is expected to have an impact on the wind component as well. Examining the surface structure based a set of intensity change stratifications will be an important piece to the puzzle as results from future analyses could be connected with results obtained from precipitation studies (Zagrodnik and Jiang 2014; Tao and Jiang 2015). 


\section{EXAMINATION OF SURFACE WIND ASYMMETRIES IN TROPICAL}

\section{CYCLONES: PART I. GENERAL STRUCTURE AND WIND SHEAR IMPACTS}

\subsection{Abstract}

In this study, global rain-corrected scatterometer winds are used to quantify and evaluate characteristics of tropical cyclone surface wind asymmetries using a modified version of a proven aircraft-based low wavenumber analysis tool. The globally expanded surface wind dataset provides an avenue for a robust statistical analysis of the changes in structure due to tropical cyclone intensity, deep-layer vertical wind shear, and wind shear's relationship with forward storm motion. A presentation of the quantified asymmetry indicates that wind shear has a significant influence on tropical storms at all radii but only for areas away from the radius of maximum wind in both non-major and major hurricanes. It was discovered that in tropical cyclones experiencing effects from wind shear, an increase of absolute angular momentum transport occurs downshear with preference for the downshear-right quadrant. The low wavenumber maximum in turn forms downwind of this momentum transport. Evaluation of shear's directional relation to motion indicates that a cyclonic rotation of the surface wind field asymmetry from downshear-left to upshear-left occurs in conjunction with an anticyclonic rotation of the directional relationship (i.e. from shear direction to the left, same, right, or opposite of the motion direction). These results are also consistent with the downwind location of the low wavenumber maximum in relation to the increased absolute angular momentum transport. 


\subsection{Introduction}

Because surface wind speeds are directly connected to TC intensity, certain factors known to impact intensity by definition should in turn impact surface wind field structure. Large-scale environmental factors significantly contribute to a TC's ability to form, intensify, and rapidly intensify (Kaplan and DeMaria 2003; Hendricks et al. 2010; Kaplan et al. 2010). Some components, such as sea surface temperature (SST), play a fairly obvious role in the intensification process (Gray 1979; Schade and Emanuel 1999). The forward motion impact on TC intensity is also well understood and well documented in terms of synoptic scale interactions, vorticity, and beta effect (Rossby 1948; Kuo 1969; George and Gray 1976; Jones 1977; Brand et al. 1981; Holland 1983a, 1984; Chan and Williams 1987; Fiorino and Elsberry 1989; Gonzalez et al. 2015), of vertical mass transport and motion (Jorgensen et al. 1985; Marks et al. 1992), of observed precipitation or convective inner-core features (Miller 1958; Willoughby et al. 1984; Marks 1985; Burpee and Black 1989; Franklin et al. 1993; Rodgers et al. 1994; Black et al. 1997; Corbosiero and Molinari 2003; Lonfat et al. 2004; Chen et al. 2006, hereafter referred to as C06), and supported by numerical simulations (Shapiro 1983; Frank and Ritchie 1999; Thomsen et al. 2015).

Other environmental factors, such as deep-layer $(850-200 \mathrm{mb})$ vertical wind shear, are less well defined in their impact on TC formation and intensification. Generally, increasing wind shear tends to inhibit TC formation or strengthening (Gray 1968), but there is evidence that supports promotion of these processes in the presence of moderate to strong wind shear (Reasor et al. 2004; Molinari and Vollaro 2010; Reasor and Eastin 2012; Reasor et al. 2013; Rogers et al. 2015). Because vertical wind shear is 
variably dependent on synoptic scale factors and on the location within a particular basin, the relationship between wind shear and storm motion has implications for determining the location and magnitude of the maximum surface wind speed (Corbosiero and Molinari 2003; Rogers and Uhlhorn 2008; Ueno and Bessho 2011 [henceforth referred to as UB11]; U14). Additionally, vertical wind shear and storm motion significantly contribute to defining the symmetric or asymmetric structure of the boundary layer and surface wind fields (S83; Kepert 2001; Ueno and Kunii 2009 [henceforth, referred to as UK09]).

Tropical cyclone penetrating aircraft are generally the standard for providing the most accurate and pertinent information needed for determining a TC's strength (Aberson et al. 2006) due to observations from SFMR (Uhlhorn et al. 2007; Klotz and Uhlhorn 2014) and GPS dropsondes (Hock and Franklin 1999; Franklin et al. 2003). When available, the observations from these instruments strongly influence the resulting 'best track' data (Jarvinen et al. 1984; Landsea and Franklin 2013). Because of proximity or resource issues, these aircraft data are generally only available over the North Atlantic basin and rarely over the Eastern Pacific. Only $\sim 30 \%$ of all six hour periods over the Atlantic have aircraft data available (Rappaport et al., 2009).

Several studies have examined the viability of using microwave instrumentation (Goodberlet et al. 1989; DeMuth et al. 2004, 2006; Bessho et al. 2006; Brennan et al. 2009; Knaff et al. 2011; Mai et al. 2016) and infrared imagery (Dvorak 1975; Mueller et al. 2006; Velden et al. 2006; Kossin et al. 2007) from space to obtain an estimate of the surface wind speeds in TCs. However, studies pertaining to scatterometry (Brennan et al. 2009; Stiles et al. 2010; Stiles and Dunbar 2010; Stiles et al. 2014) provide the most 
direct estimation of the surface wind speeds from space. Scatterometers such as QuikSCAT (Draper and Long 2002, 2004; Hoffman and Leidner 2005) and ASCAT (Figa-Saldaña et al. 2002) have been particularly useful for determining operational wind radii, but they are generally disregarded near the inner-most radii of TCs.

Scatterometer wind speeds are generally trustworthy up to $\sim 30 \mathrm{~m} \mathrm{~s}^{-1}$ in TCs (Brennan et al. 2009; Stiles et al. 2014). The lack of hurricane force wind speed observation in hurricanes is a significant problem for TC analysis and is compounded by interference of the microwave signal from precipitation. However, it is possible to apply a correction to these wind speeds using a Neural Network (NN) in order to attain wind speeds up to $\sim 55-60 \mathrm{~m} \mathrm{~s}^{-1}$ ( $\pm 1-2 \mathrm{~m} \mathrm{~s}^{-1}$, Stiles et al. 2014). While the NN correction method is one of several available, it was developed with the assistance of an operational product (H*WIND, Powell and Houston 1996; Powell et al. 1998) and substantially reduces the impact of precipitation on the wind speed result. Therefore, utilizing it increases the wind speed reliability in most conditions.

With improved wind speeds, more attention can be placed on the evolution of the surface wind asymmetry as it pertains to several environmental and storm specific parameters. Because over a decade worth of this global data ( 2000-present) exists for the full TC intensity spectrum, it is possible to provide composite analyses of the surface wind asymmetric structure for various stratifications. For winds, the asymmetric structure is best examined using a low wavenumber analysis as shown in U14, which lays the foundation for observed TC surface wind structure and provides a general idea of the surface wind asymmetry. Because of their limited dataset size, it is difficult to provide 
statistically robust composites of the surface wind asymmetric structure in any greater detail than already provided.

Ueno and Bessho (2011) examined surface wind asymmetries from scatterometer winds in terms of storm motion and wind shear, finding a preferential left-of-shear and right-of-motion maximum. Because they use rain-flagged data, their sample may be contaminated. The justification for using rain-flagged data was that their work was more of a "feasibility study on the utility of the data in the TC near-core region". Klotz and Jiang (2016, herein referred to as KJ16) indicate that the scatterometer wind field evolves as previous modeling studies have shown (S83; Thomsen et al. 2015) but opens the door for further analysis through quantification of the surface wind asymmetry using a large and robust dataset. Because of the lack of detail provided in previous surface wind studies, the present study is unique and seeks to determine the low wavenumber, surface wind asymmetry from NN corrected scatterometer data in a similar manner to U14 while also expanding on the initial results of KJ16. Quantification and a description of the possible mechanism(s) controlling the low wavenumber surface wind asymmetry in the presence of wind shear is also a focus of this work. The sections are presented as follows: a data description will be provided in section 3.3, followed by a description of the low wavenumber method applied to the scatterometer winds in section 3.4. A fifth section includes results and discussion of the surface wind asymmetry as a function of TC intensity, shear and motion angle difference, and seeks to provide evidence to explain their variations. A final section provides conclusions to this study. 


\subsection{Data}

\subsubsection{Satellite-based scatterometer data}

With a fairly large footprint size and swath length (i.e., $\sim 25 \times 31 \mathrm{~km}$ and $\sim 1800$, respectively, for QuikSCAT), scatterometers are useful for examining the full TC wind field, which is an advantage when compared to data collected by aircraft. As mentioned previously, JPL maintains a dataset (Hristova-Veleva et al. 2013) that contains the TCcentric QuikSCAT and OSCAT data that are available for all TC-prone basins between 2000-2011 and are processed with a horizontal resolution of $12.5 \mathrm{~km}\left(\sim \frac{1}{8}^{\circ}\right.$, Brennan et al. 2009). While the scatterometer often observes a full TC wind field, lack of targeting by the satellite prevents guaranteed coverage of a TC during an overpass. As a reminder, all scatterometer wind speeds used herein will have the NN correction applied (Stiles et al. 2014) to reduce contamination from rain.

\subsubsection{Airborne SFMR data}

In order to validate scatterometer winds and analyses used in this study, SFMR wind speeds and their resulting analyses are utilized. The SFMR uses a stepping technique through six $\mathrm{C}$-band frequencies to determine a surface wind speed and rain rate from the six observed brightness temperatures. Data are provided at a rate of $1 \mathrm{~Hz}$, but a set of completely independent brightness temperatures occurs every $0.1 \mathrm{~Hz}$ (Uhlhorn and Black 2003, Uhlhorn et al. 2007). These SFMR data are often a determining factor in operational intensity estimates because they are a trustworthy source of surface wind speed observation within a TC (when available). Twenty-four overlapping cases from the SFMR dataset in U14 are utilized for validation of scatterometer data. 
Despite the advantages of aircraft reconnaissance, there are also caveats that must be considered when using these data. A limiting factor here is the SFMR high bias of wind speed in the presence of heavy rain (mostly for weaker systems). This bias problem has been addressed (Klotz and Uhlhorn 2014), but the SFMR data in the present study have not been reprocessed with this correction. Because the cases used in U14 were hurricanes and because the SFMR performs well at high wind speeds (mean bias $<1 \mathrm{~m} \mathrm{~s}^{-1}$, Klotz and Uhlhorn 2014), the lack of this bias correction will not impact the validation of the scatterometer. Therefore, the SFMR data are useful for validating the analyses produced from scatterometer winds.

\subsubsection{TC Intensity, Motion, and Vertical Wind Shear Data}

For TC position, official intensity, and storm motion parameters, the hurricane best track provides 6-hourly data and is currently available in Hurricane Database 2 (HURDAT2, Landsea and Franklin 2013) for the North Atlantic and Eastern North Pacific basins through the 2015 season. Similar files are provided by the JTWC for the remainder of the TC basins. For the current study, TC location and 6-hour motion vector along with the maximum wind speed are co-located in time with each scatterometer file. For reasons similar to using best track data, vertical wind shear data are obtained from the SHIPS database (DeMaria and Kaplan 1994, 1999) for the same periods as the best track data. Shear is calculated for a deep layer (between $850 \mathrm{mb}$ and $200 \mathrm{mb}$ ), and $500 \mathrm{~km}$ radially outward of the TC center, the vortex has been removed. Data are available for the entire study period for all basins with the exception of the first few storms of 2000 for the Northwest Pacific basin. Vertical wind shear data are provided at 6-hourly intervals in the same manner as the best track data. Discussion of the practicality of using a shallow shear 
layer is discussed briefly in KJ16 (Chapter 2), where they determined the deep layer shear is suitable for the purpose of the current study.

\subsection{Methods for Producing Low Wavenumber Analyses with Scatterometer Data}

\subsubsection{Low Wavenumber Analysis}

To determine and quantify motion and shear-dependent low wavenumber asymmetric surface wind structure using global scatterometer data, the present study expands on previous work from Vukicevic et al. [(2014), herein referred to as V14] and U14 that examined surface wind asymmetry from SFMR and model data. In V14, a Fourier decomposition method for determining the low wavenumber field is written as:

$$
V(r, \theta)=V_{0}(r)+V_{1} \cos \left(\theta-\alpha_{1}(r)\right)+\varepsilon(r),
$$

where $V_{0}$ and $V_{l}$ are the wavenumber- 0 and -1 amplitude, respectively, $\alpha_{l}$ is the wavenumber-1 phase, and $\varepsilon$ is the total remaining contribution of the higher order wavenumbers. This $V$ in Eq. 3.1 is defined on a polar coordinate reference frame, where $r$ and $\theta$ are the radial and azimuthal coordinates, respectively. At the RMW and associated azimuth, Eq. 3.1 becomes the representative of $V_{\max }$ (TC intensity).

Low wavenumber analyses for the scatterometer are processed in a similar fashion to the SFMR described in U14, with the exception that there is better azimuthal coverage. After standardizing the radial grid as described in Chapter 2, the azimuthal components to these locations are then combined with the wind data in a similar form to Eq. 3.1. An unconstrained nonlinear optimization function that minimizes the error between the function-determined values and the wind speed observations is then used to determine the Fourier parameters for wavenumber- 1 at each radial bin. While the focus of this work is applied to wavenumber-1, higher order wavenumbers are resolvable if there 
are enough degrees of freedom present, which is dependent on the percentage of swath coverage in the inner-core. The wavenumber amplitudes and phases $\left(V_{n}\right.$ and $\alpha_{n}$, where $\mathrm{n}$ represents wavenumber beginning at 0 ) are then calculated using the following equations:

$$
\begin{aligned}
& V_{n}=\sqrt{\left(s_{(n \times 2)}^{2}+s_{(n \times 2)+1}^{2}\right)} \\
& \alpha_{n}=a \tan 2\left(s_{(n \times 2)+1}, s_{(n \times 2)}\right)
\end{aligned}
$$

In Eq. 3.2, $s_{n}$ is the parameter determined by the optimization function. For solving to wavenumber-1, $V_{0}=s_{1}, V_{1}=\sqrt{\left(s_{2}^{2}+s_{3}^{2}\right)}$, and $\alpha_{1}=a \tan 2\left(s_{3}, s_{2}\right)$.

The above description references an earth-relative framework, where the motion of the storm has not been removed from the wind vectors. From simple vector geometry, it is well known that winds are generally higher on the right (left) side of the motion vector for the Northern (Southern) hemisphere when direction is oriented toward the respective pole. This result was verified for scatterometer winds in KJ16 (Chapter 2). By removing the storm translation contribution to the winds, the true wind speed asymmetry is assessable in a storm-relative framework. Following the analysis methods above, low wavenumber analyses are producible using the wind vectors in various frameworks (i.e., storm- or shear-relative). For details of the cases used in this study, please consult Table A1.

\subsubsection{Compositing Procedures}

Individual analyses of surface winds serve a variety of purposes for understanding storm-specific characteristics, but by creating composite analyses, it is much easier to determine prominent features within the surface field, including the asymmetric structure. The compositing technique used in this study is straightforward by averaging the 
individual fields on the normalized radial grid. Additionally, a weighting scheme based on the swath coverage percentage within $250 \mathrm{~km}$ of the TC center is used to apply the best data and maximize the sample size. Effectively, $100 \%$ coverage within $125 \mathrm{~km}$ is required and $>70 \%$ coverage is required through $250 \mathrm{~km}$. The resulting weights are on a scale of $0-1$, and only weights $\geq 0.7$ are used. Because of this weighting scheme, KJ16 note that $>75 \%$ of the cases have a weight of at least 0.9 , so there is less likelihood of analysis problems due to lack of coverage.

For the present study, an emphasis is placed on TC intensity, storm motion, and vertical wind shear impacts on the surface wind asymmetry. Composites of these stratifications are computed for the full dataset. Because cases from the Southern Hemisphere are included in the dataset, it is important to rotate these low wavenumber analyses to a Northern hemisphere frame of reference (as in C06) in order to apply them correctly in the composite calculation and for comparison against the other basins. Further examination of motion and shear impacts are computed by comparing all shear conditions to weak shear $\left(V_{s h r}<3.2 \mathrm{~m} \mathrm{~s}^{-1}\right)$, as well as the angle difference between the motion and shear vectors. The cutoffs for shear and motion are determined from the lower and upper $17.5 \%$ of the cumulative distribution function (CDF) of the respective variable, and they are comparable to those determined in C06. Angle differences $\left(\Delta \theta_{s m}=\right.$ $\left.\theta_{s h r}-\theta_{\text {storm }}\right)$ also follow the methodology of $\mathrm{C} 06$, where $\left|\Delta \theta_{s m}\right| \leq 22.5^{\circ}$ and $\left|\Delta \theta_{s m}\right| \geq 157.5^{\circ}$ are designated as same and opposite, respectively. Angle differences for $22.5^{\circ}<\Delta \theta_{s m}<$ $157.5^{\circ}$ and $-157.5^{\circ}<\Delta \theta_{s m}<-22.5^{\circ}$ are designated as right and left, respectively. 


\subsection{Results and Discussion}

\subsubsection{Validation of the Scatterometer Analysis Method Using SFMR Data}

The first goal of this study is to determine if the low wavenumber analyses of scatterometer data resemble the analyses from SFMR. It has been established that the resolution of the scatterometer data is coarser than the SFMR, and this will ultimately affect the radial component of the analyses. Increased uncertainty in the scatterometer winds at higher wind speeds is indicative of increased differences between the maximum wavenumber- $0+1$ amplitude $\left(V_{0}+V_{1}\right.$ or $\left.V_{0+1}\right)$ of the two wind sources. A statistical examination of a set of coincident cases is used to provide more substance to the results from KJ16. Table 3.1 provides a list of cases along with the scatterometer-relative offset time for SFMR.

Table 3.1. A compiled list of coincident SFMR and scatterometer cases is provided and separated based on their best-track intensity. In parentheses is the SFMR offset in hours relative to the scatterometer time.

\begin{tabular}{|c|c|c|}
\hline Storm Category & Number of cases & Storm names (year, SFMR offset) \\
\hline TS & 1 & Katrina $(2005,+2)$ \\
\hline 1 & 4 & $\begin{array}{c}\text { Alex }(2010,-1), \text { Igor }(2010,-4), \operatorname{Karl}(2010,+3), \\
\text { Rina }(2011,-3)\end{array}$ \\
\hline 2 & 3 & Felix $(2007,-2)$, Danielle $(2010,-4)$, Igor $(2010,-4)$, \\
\hline 3 & 5 & $\begin{array}{c}\text { Bill }(2009,-3) \text {, Frances }(2004,+3), \text { Gustav }(2008,- \\
\text { 4), Ivan }(2004,+4), \text { Rina }(2011,+5)\end{array}$ \\
\hline 4 & 9 & $\begin{array}{l}\text { Frances }(2004,+4) \text {, Danielle }(2010,-4) \text {, Earl }(2010,- \\
1,0 \text {, and }+1) \text {, Ivan }(2004,-2) \text {, Isabel }(2003,+5) \text {, Rita } \\
(2005,+3 \text { and }+3)\end{array}$ \\
\hline 5 & 2 & Ivan $(2004,+5)$, Katrina $(2005,+5)$ \\
\hline
\end{tabular}

In Figure 3.1(a), maximum $V_{0}+V_{l}$ for the scatterometer winds are plotted as a function of the coincident SFMR values, and the weighted linear regression fit is provided for comparison against a perfectly correlated dataset. Several amplitude pairs 
were removed from the statistical comparison because they exist outside the two standard deviation threshold of the amplitude differences $\left(\delta V=V_{s f m r}-V_{s c t}\right)$. Note that the shaded markers indicate the weight associated with the scatterometer data and that the text displayed in (a) is associated with weighted values of $\delta V$. The scatterometer maximum amplitude compares well to the SFMR values as indicated by the weighted regression fit, and as expected, there is a greater tendency to underestimate the SFMR amplitude above $50 \mathrm{~m} \mathrm{~s}^{-1}$. Additionally, a weighted and paired student's t-test indicates that these results are statistically significant at $95 \%$.

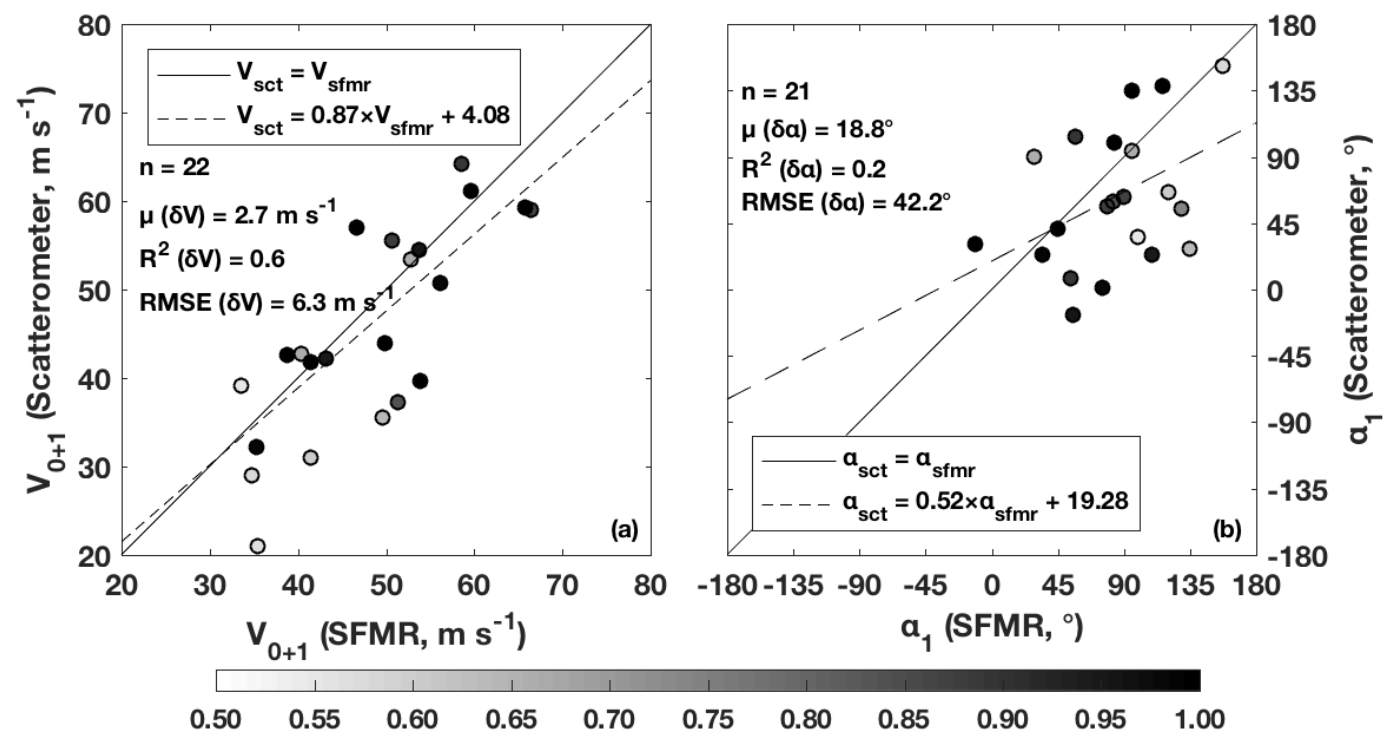

Figure 3.1. In (a), maximum scatterometer wavenumber- $0+1$ amplitudes $\left(V_{s c t}\right)$ are plotted as a function of coincident ( $\pm 5 \mathrm{~h}$ of scatterometer time) maximum SFMR wavenumber$0+1$ amplitudes $\left(V_{s f m r}\right)$. Varying shades of gray of the markers indicate the weight applied to the scatterometer swath. A weighted linear regression fit is displayed (dashed line) using the weights associated with each marker. In (b), the associated wavenumber-1 phase at the maximum amplitudes are plotted in a similar manner to (a), where the text statements represent the weighted statistical values of the difference between phase angles $\left(\delta \alpha=\alpha_{s f m r}-\alpha_{s c t}\right)$ and the dashed line indicates the weighted linear regression fit.

Figure 3.1(b) provides the scatter of $\alpha_{1}$ at the maximum $V_{0}+V_{l}$ for the scatterometer winds as a function of the associated SFMR $\alpha_{1}$. Outliers of these data were 
removed using the weighted, two standard deviation threshold of the phase angle difference $\left(\delta \alpha=\alpha_{s f m r}-\alpha_{s c t}\right)$. The weighted linear regression fit to these data is also provided. A plausible reason for the difference in phase is the superior azimuthal coverage of the scatterometer data. The types of patterns flown for SFMR (Uhlhorn and Nolan 2012) require a minimum of two center passes (four radial legs) to calculate wavenumber components. This reduced coverage could lead to some assumptions about the winds in unobserved portions of a TC. On the other hand, the scatterometer data are available at a wider range of azimuths and observe similar magnitude wind speeds, implying that the location of the asymmetry may be more credible in this case, especially for larger storms. These results are statistically significant within the $95 \%$ confidence interval as well. The statistical results for both amplitude and phase confirm that the scatterometer is capable of providing reliable analysis and evaluation of the surface wind asymmetry.

\subsubsection{Asymmetric Structure as a Function of TC Intensity}

The surface wind asymmetry is most clearly depicted as a dependence on storm intensity. Figure 3.2 displays the global composite analyses for tropical depressions and storms, Category 1-2 (non-major) hurricanes, and Category 3-5 (major) hurricanes in (ac), respectively. These composites are normalized based on the maximum amplitude of the composite (on a scale of $0-1$ ) and are plotted on a normalized radial grid in a sheardirection-relative orientation (with storm motion removed). Note that the maximum low wavenumber amplitude (black marker) tends to be located left-of-shear (LS) for tropical storms and non-major hurricanes while major hurricanes have their maximum located more downshear (DS). For reference, shear-relative locations will be abbreviated in the 
remainder of the study, where downshear-left is now DSL or upshear-right is USR. From a glance at the fields it is clear that the weaker systems have a more pronounced asymmetric structure overall. Note that these composites are statistically significant at $95 \%$ when compared to the overall global composite.
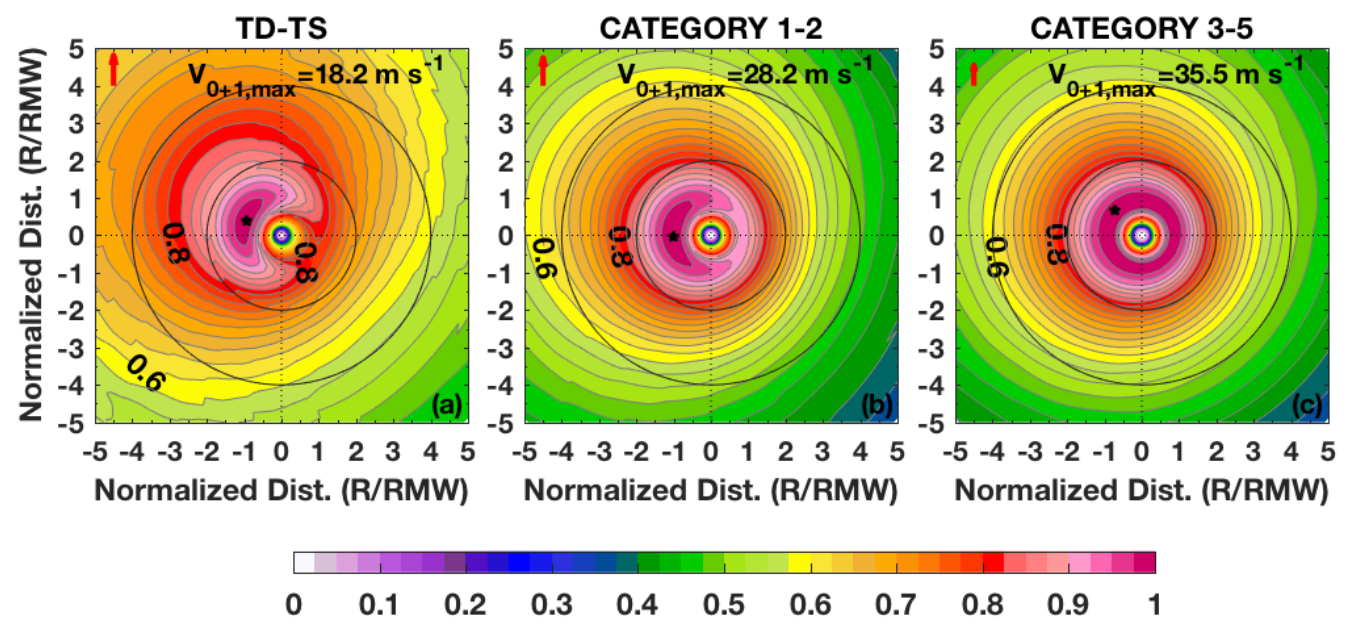

Figure 3.2. Normalized composite shear-relative (with motion removed) wavenumber$0+1$, two-dimensional scatterometer wind speed analyses as a function of normalized radius are provided for (a) tropical depressions and storms, (b) Category 1 and 2 hurricanes, and (c) Category 3-5 hurricanes. The red arrows show the direction of shear and the black marker indicates the location of the maximum amplitude. Contours and colors are plotted every 0.025 normalized units. These results are significant at $99 \%$.

Going a step further, U14 examined the low wavenumber amplitude and phase at the RMW with respect to storm motion and wind shear speed (see their Figures 8 and 10). Their results indicate that increasing the storm motion is associated with an anticyclonic rotation of the phase from down- to right-of-motion (DM to RM) while increasing the shear speed rotates the phase from DS to LS. Note that for the motionrelative results, the motion vector has not been removed from the low wavenumber analysis. Figure 3.3 in the current study provides a similar analysis but separated into TC intensity groups. 

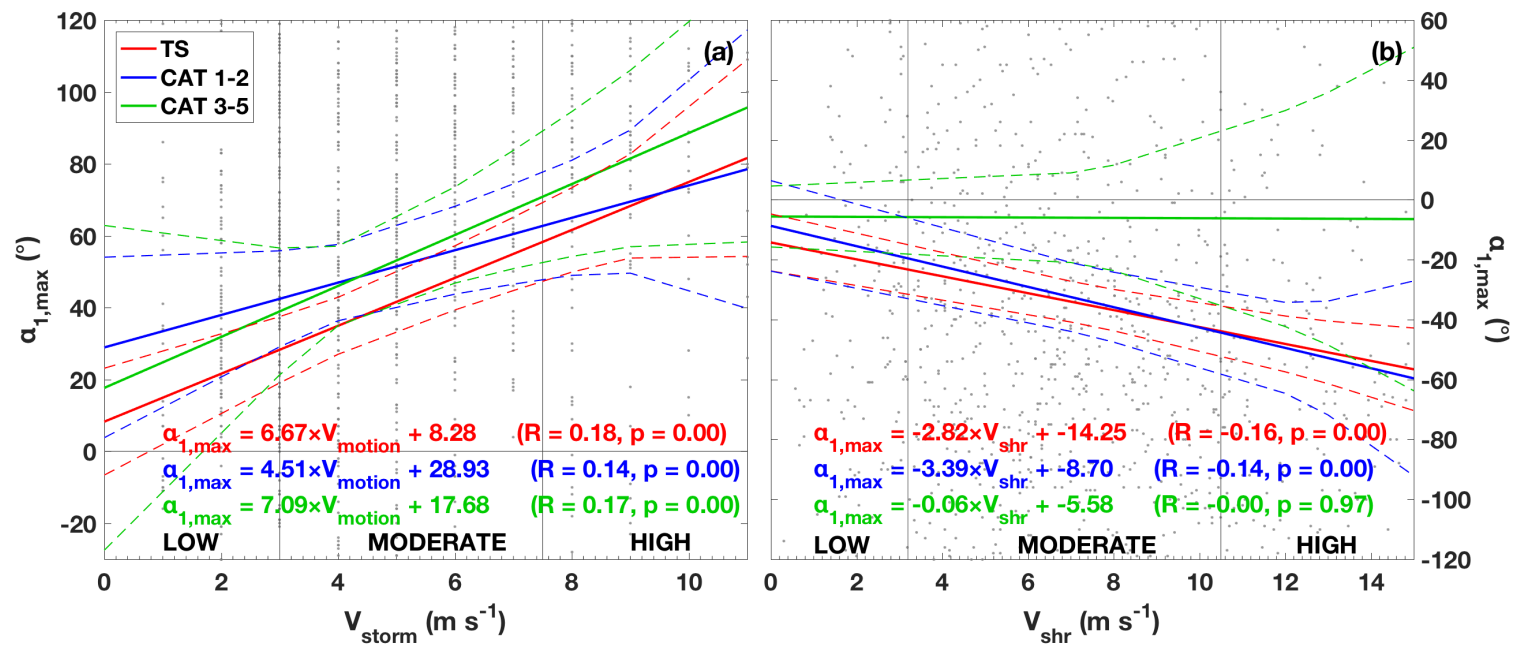

Figure 3.3. In (a), linear regression fits of the wavenumber-1 phase at the maximum motion-relative amplitude $\left(\alpha_{1}, \max \right)$ are provided for three intensity groups as a function of storm speed $\left(V_{\text {storm }}\right)$. Similar fits are shown in (b) but in a shear-relative (motion removed) reference frame and as a function of vertical wind shear speed $\left(V_{s h r}\right)$. For reference, the zero line is indicative of the down-motion or down-shear direction, with negative phase angles representing locations to the left of motion or shear. The gray markers are the $\alpha_{1}$, max values associated with the tropical depression and storm cases. Dashed lines indicate the $95 \%$ confidence interval of the respective fit.

In Figure 3.3(a), the motion dependence is similar to U14 where $\alpha_{1}$ rotates from $\mathrm{DM}$ to $\mathrm{RM}$ with increasing $V_{\text {storm }}$. Interestingly, non-major hurricanes have the lowest correlation with increasing motion, while major hurricanes have the largest impact from storm motion on the rotation of the asymmetry. It is generally assumed that an additional asymmetry $90^{\circ}$ to the right of motion should be applied to analyses of vortex structure, but Figure 3.3(a) confirms U14's suggestion that this is not always a correct assumption. UK09 additionally suggests that the maximum wind (and effectively the low wavenumber maximum) should occur $90^{\circ}$ downwind of the maximum inflow. However, the parametric inflow angle model described in Zhang and Uhlhorn (2012) suggests that maximum inflow location near the RMW rotates cyclonically with increasing storm speed. Therefore, a cyclonic rotation of the low wavenumber maximum would be 
expected. From the results presented here and in U14, the anticyclonic phase rotation is more a result of simple vector geometry where in slow moving storms, the rotational component of the wind is controlled by internal vortex dynamics. Increasing the speed imparts an asymmetry on the winds that amplifies the maximum signal largely to the right of motion where the vectors align.

Figure 3.3(b) is similar to (a) in form but shows the change in $\alpha_{1}$ at the RMW as a function of shear speed and in a shear-relative (storm motion removed) reference frame. For the tropical storm and non-major hurricane cases, $\alpha_{1}$ rotates cyclonically from DS to LS, which is similar to U14. However, the major hurricane cases have no observed correlation with increasing shear speed. Looking back at Figure 3.2(c), there is a hint of an increased asymmetry in the DS to DSL direction, but the composite is much more symmetric than the other groups near the RMW. The low correlation could be a result of the scatterometer resolution and uncertainty at high wind speeds. It is also possible that there is some randomness to the location of the maximum amplitude in relation to shear, which could be explained by the principle of vortex resiliency (Reasor et al. 2004; Reasor and Eastin 2012). The dynamics of the rotating vortex, therefore, would maintain a symmetric system regardless of shear strength.

\subsubsection{Relative Contribution of Wind Shear on TC Surface Wind Structure}

Wind shear impact on the $\mathrm{TC}$ vortex is not a trivial factor to consider as shear has varying impacts on TCs. Quantifying the relative impact of shear on the surface wind asymmetry is a necessary next step to the results in KJ16 and U14. Using the two dimensional composites of $V$ for wavenumber- 0 and -1 , ratios of wavenumber-1 amplitude to the total low-wavenumber amplitude $\left(V_{l} / V_{0+1}\right)$ are calculated and mean 
radial profiles of this ratio are computed for each shear relative quadrant (USL, DSL, DSR, USR). Following the description in Alvey et al. (2015) and Tao et al. (2017), a low wavenumber asymmetry index (AIDX) is then determined in the following manner:

$$
A I D X=\sum_{i=1}^{4} Q_{i} \rightarrow\left\{\begin{array}{l}
Q_{1}=|U S R-D S R| \\
Q_{2}=|U S L-D S L| \\
Q_{3}=|U S L-D S R| \\
Q_{4}=|D S L-U S R|
\end{array}\right.
$$

A large asymmetry index value signifies a high degree of asymmetry. Figure 3.4 shows radial profiles of AIDX as a function of TC intensity for the combined as well as the quadrant differences. Looking at the full range of shear conditions (solid lines), the TS cases have the largest AIDX values at every normalized radius in panel (e). It is clear to see that a majority of the contribution is coming from the absolute difference between the DSL and USR quadrants in panel (d). Both hurricane groups have successively lower asymmetry indices at all radii in these quadrants compared to the TS cases, but the gradient of AIDX is much larger between 1.0-2.5 $\times$ RMW. For comparison, AIDX within this radial range increases by $\sim 1 / 3$ of the RMW value for tropical storms while it nearly doubles to triples for non-major hurricanes.

The change for major hurricanes follows a similar trend, but the difference is more pronounced, as AIDX is nearly 10 times larger at $2.5 \times \mathrm{RMW}$ than at the RMW. Near the RMW in Figure 3.4(e), the tropical storm cases are three times more asymmetric than the non-major hurricanes (and at least 15 times more asymmetric than major hurricanes). All differences in asymmetry described here are statistically significant at $95 \%$. These results indicate that tropical storms are generally asymmetric through most 
radii while hurricanes exhibit some degree of symmetry in the inner region of the vortex before succumbing to other significant influences (shear, remaining motion impacts, or low-level local flow) with increasing radii.
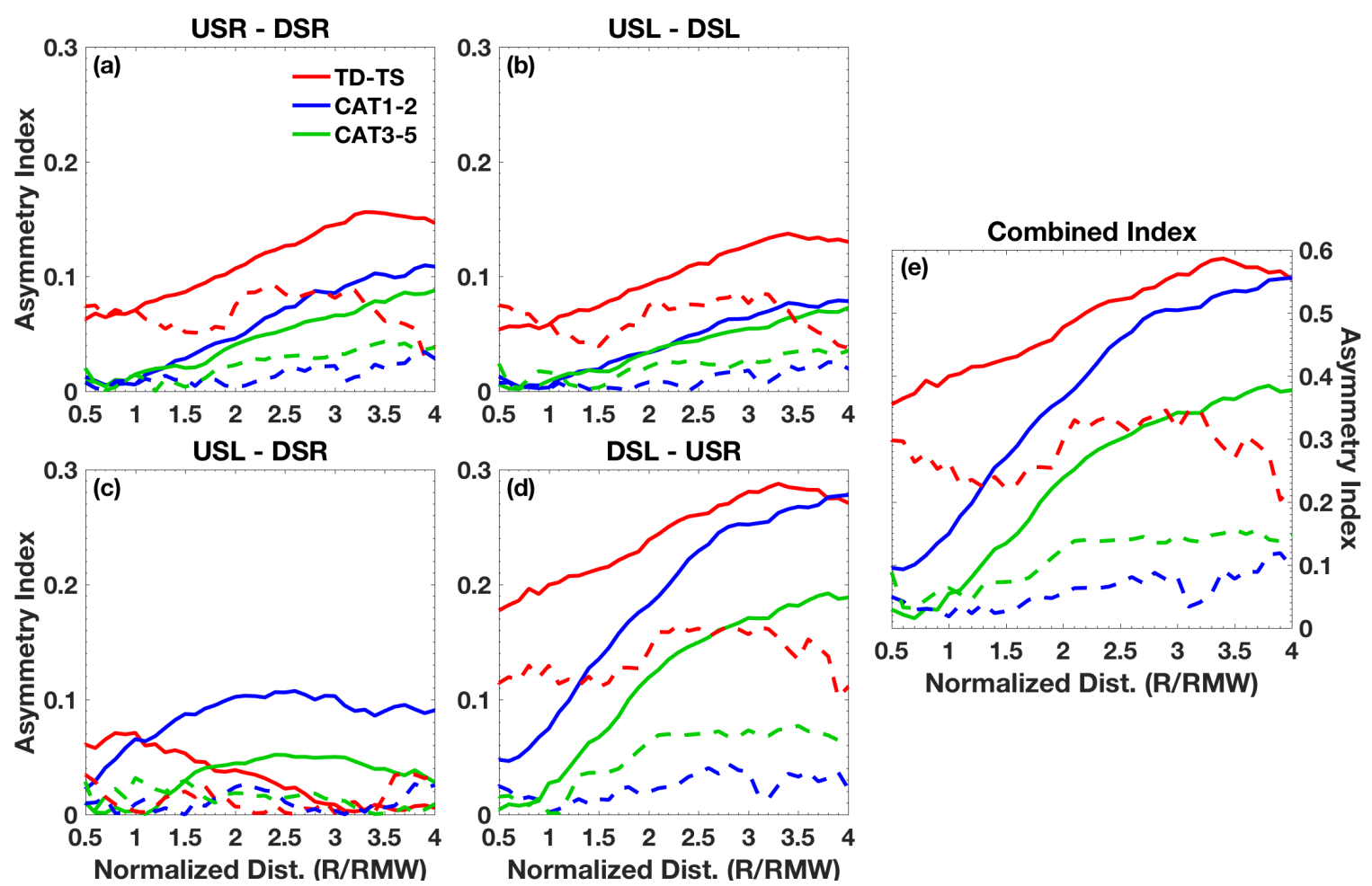

Figure 3.4. In panels (a-d), TC intensity dependent radial profiles of wavenumber-1 asymmetry ratio $\left(V_{l} / V_{0+1}\right)$ are provided in the form of the four shear-relative quadrant absolute differences that define an asymmetry index (Alvey et al. 2015; Tao et al. 2017). 'DS' and 'US' refer to down-shear and up-shear and the additional ' $L$ ' or ' $R$ ' indicates right or left, respectively. In (e), the total asymmetry index is provided similarly to (a-d), where larger values indicate more asymmetry. Dashed lines display the same quantities but for low shear conditions only $\left(V_{s h r}<3.2 \mathrm{~m} \mathrm{~s}^{-1}\right)$.

By separating cases that experience low shear (452 cases), it is possible to compare with the results of full range of shear and determine the shear's relative impact on the surface wind speed asymmetric structure. Figure 3.4 displays these low shear radial profiles (dashed lines in all panels) in a similar fashion to those shown in using the full range of shear values. Looking first at the region near the RMW for the full AIDX, the major hurricane cases are nearly unchanged after reducing the shear, which quantifies 
the authors' hypothesis that the inner-most portion of major hurricanes are not experiencing much impact from shear in terms of the surface asymmetry. Tropical storms, however, experience an asymmetry reduction (on the order of $25-50 \%$ ) near the RMW. After significantly reducing the shear impact on the vortex, tropical storm cases maintain a higher degree of asymmetric structure, which indicates that shear and residual factors are both important contributors for these weak systems. Interestingly, non-major hurricanes see the largest impact when reducing the shear magnitude, where near the RMW, AIDX is reduced by a factor of three. It is evident that wind shear is a significant contributor to their asymmetric structure and that residual factors play less of a role. Outside of the RMW (radius $>1.5 \times \mathrm{RMW}$ ), the asymmetric structure of tropical storms is clearly impacted by shear, where AIDX is reduced by at least half at all radii. This reduction is more apparent in the non-major (major) hurricanes with asymmetry ratios reduced in magnitude by upwards of six (two to four) times the full shear profiles. Both hurricane groups are indicative of an asymmetric surface wind field that is highly dependent on wind shear outside the RMW.

Klotz and Jiang (2016) suggested the possibility that non-major hurricanes represent a transition stage between which shear and residual impacts both serve a prominent role in determining the asymmetric structure. Using the AIDX profiles, this hypothesis seems justifiable near the RMW as the asymmetry induced by shear steadily decreases with increasing intensity [Figure 3.4(d) and (e)]. However, this idea does not appear to hold true outside the RMW. If shear is the main contributor to asymmetry, reducing the shear should significantly reduce the wavenumber-1 asymmetry, which is generally what is portrayed in both hurricane groups. However, tropical storms still 
exhibit a prominent asymmetric structure after reducing the shear. To help diagnose a possible explanation for the maintenance of the asymmetry, the change in absolute angular momentum (AAM) transport between the intensity groups should be revealing. A modified form of the initial AAM equation is given by Pálmen and Riehl (1957), but for the purposes of this study, quadrant specific radial profiles of AAM flux are calculated using a modified version of equation (4) from Chan and Chan (2013), which takes the following form:

$$
A A M F(r)=r \bar{v}_{\theta} \bar{v}_{r}+r \overline{v_{\theta}^{\prime} v_{r}^{\prime}}+\frac{1}{2} f r^{2} \bar{v}_{r}+\frac{1}{2} r^{2} \overline{f v_{r}^{\prime}}+F
$$

Each quadrant is defined on the shear-relative range from $[-\pi \pi]$ with increments of $\pi 2$ radians and $\mathrm{r}$ is defined on the range of $0-250 \mathrm{~km}$ with $6 \mathrm{~km}$ radial increments. The frictional term $(F)$ is needed for momentum conservation purposes. Terms with overbars represent quadrant averages and those with primes represent perturbations from the quadrant mean. Chan and Chan (2013) refer to the first two terms on the right side as the symmetric and asymmetric relative AAM flux (i.e., SRAM and ARAM flux), respectively. The last two terms on the right side represent the symmetric and asymmetric Coriolis torque (SCT and ACT). Holland (1983b) provides a detailed examination of the contribution of these terms and their influence on the TC structure. He describes that SRAM and ARAM flux both import momentum towards the TC center, counteracted by frictional dissipation and an anticyclonic acceleration of momentum with increasing radius imparted by the SCT term. ACT is noted to only influence the circulation well away from the TC center. The variable names will be used as in Chan and Chan (2013), but in the quadrant-based framework, they are not truly symmetric or asymmetric as 
originally described due to the fact they do not refer to an entire $360^{\circ}$ field. The purpose of separating AAM flux into quadrants is to examine changes in relation to the wavenumber-1 asymmetry.

Quadrant profiles of near surface AAM flux are shown in Figure 3.5 in a shearrelative reference frame. It should be noted that we omit the contribution from friction, which tends to counter the SRAM flux, in order to highlight the positive contributions to spin up near the surface. The tropical storm group does not have a strong peak in AAM flux in any quadrant, but the flux is higher in the DSL and DSR quadrants compared to the US quadrants. Reducing the shear also reduces the magnitude of the AAM flux DS but increases the impact in the US quadrants. Shear in this instance induces an asymmetry, although weak, in the AAM transport that is maximized DSR.

For both hurricane groups, the AAM flux asymmetry is significantly decreased when shear is lowered, especially outside the RMW and in the US quadrants for nonmajor hurricanes. In the DSR quadrant, a larger amount of AAM transport occurs compared to the other three quadrants, which is most apparent with major hurricanes. At lower values of shear, the AAM fluxes are more symmetrically distributed because of the consistent AAM flux decrease (increase) in the DSL and DSR (USL and USR) quadrants, coincident with a more symmetric low wavenumber field. Interestingly, convective updrafts tend to be triggered DSR (Hence and Houze 2011). The increased AAM transport DSR overlaps a region of increased surface convergence near the RMW, which following Hence and Houze (2011), seems to promote a region of developing convection that ultimately rotates cyclonically and matures in the DSL quadrant. It is unclear how the two are related, but the DSR AAM flux maximum could be tied to the convective 
processes. It is also possible that this maximum AAM flux DSR is connected to a low level environmental flow (Reasor et al. 2013).
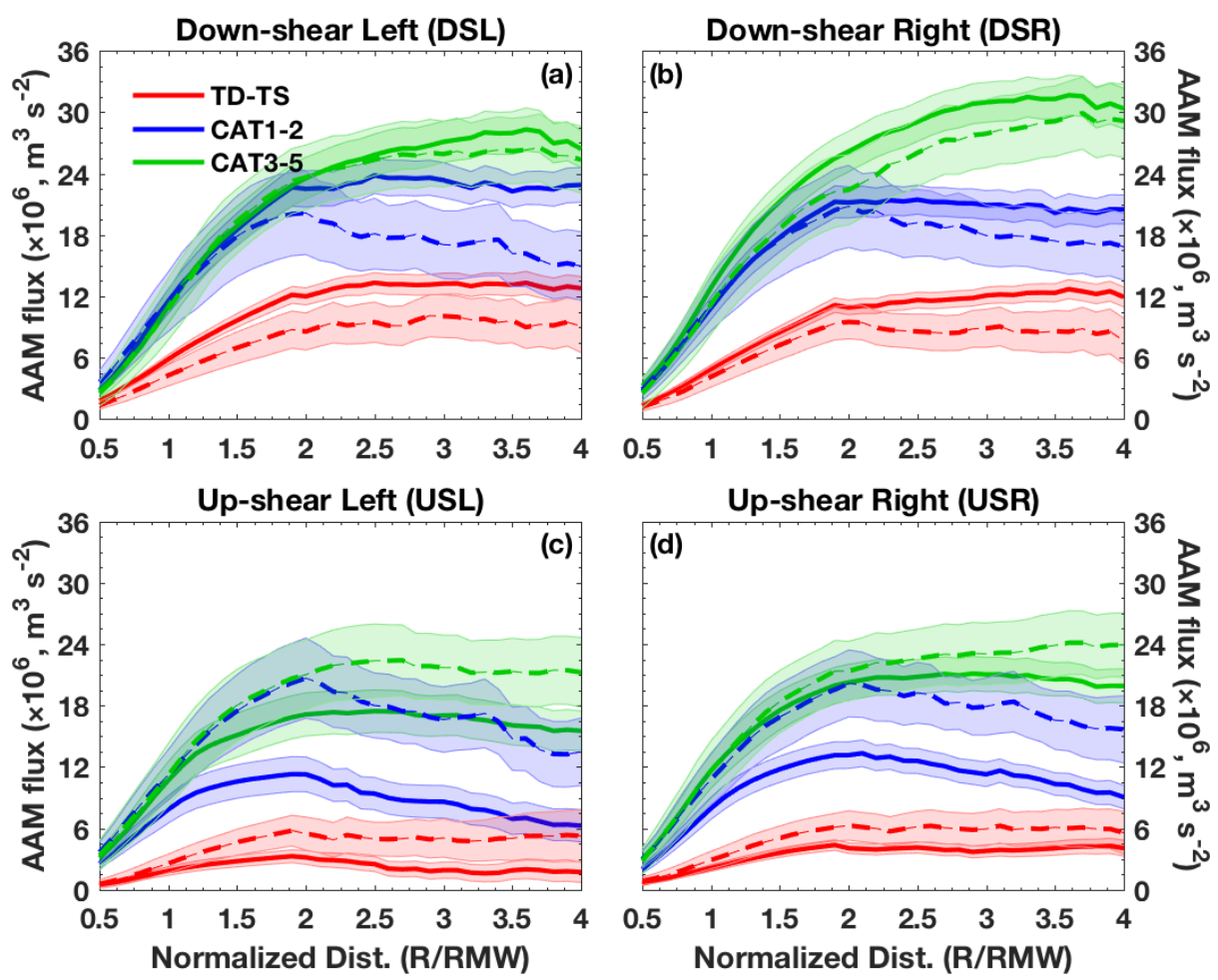

Figure 3.5. Shear-relative quadrant profiles of surface absolute angular momentum flux $\left(1 \times 10^{6} \mathrm{~m}^{3} \mathrm{~s}^{-2}\right)$ as a function of TC intensity are provided for the full range of shear values (solid lines) and for low shear conditions (dashed lines). The envelopes surrounding the lines represent the $95 \%$ confidence intervals.

The idea of shear impacting the surface AAM transport is exemplified when studying the individual contributors to the flux equation. Figure 3.6 shows the various quadrant profiles for the different intensity groups (columns) and three of the parameters (rows). The ACT term is negligible here (Holland 1983b) compared to the other three terms and thus is not discussed. Notice that as intensity increases, SRAM flux clearly dominates the total AAM flux before reducing the shear, especially DSR. In these profiles, shear influences the vortex outside the RMW in a way that increased transport of AAM is forced into the DS quadrants and generally upwind of the low wavenumber 
maximum. The ARAM flux follows similar trends as SRAM flux, but this term is negated by the SCT term within $\sim 1.5 \times$ RMW. These results support Holland (1983b) suggesting that SRAM flux is the dominant low-level process maintaining or intensifying TCs.
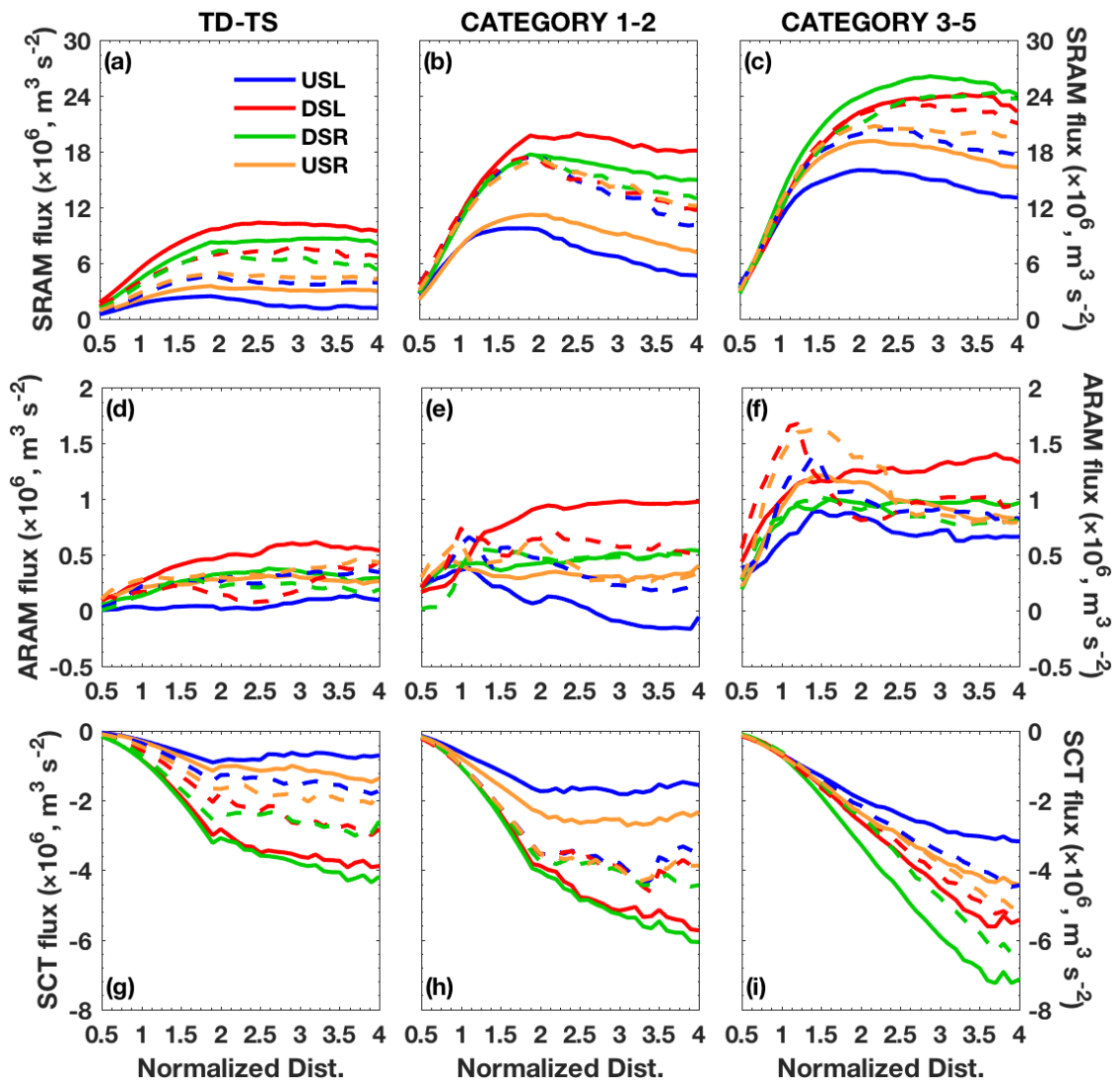

Figure 3.6. For the profiles presented in Figure 3.5, the same quadrant profiles for the individual terms of the absolute angular momentum flux equation are shown. The top and middle rows provide the symmetric and asymmetric relative angular momentum flux (SRAM and ARAM, respectively). The bottom row provides the symmetric Coriolis torque term. The three columns from left to right represent tropical storms, non-major hurricanes, and major hurricanes, respectively, and the colored lines are for the respective shear-relative quadrants.

Outside $2 \times \mathrm{RMW}$, the symmetric Coriolis term starts to act against the symmetric momentum import term. Looking back at Figure 3.5, cases experiencing moderate to strong shear are able to somewhat resist this opposing force because of the infusion of 
high AAM flow DS. However, lowering the shear dampens this increased inward momentum transport and allows the anticyclonic rotational term to have more influence on the vortex as a whole. Therefore, the presence of shear amplifies the AAM flux DS in all intensity groups and forces an increase of inward AAM transport, near and upwind of the region of maximum low wavenumber asymmetry. Relaxation of the shear in turn promotes alignment of the vortex and an increased symmetry of momentum transport as opposing forces are no longer overpowered by the AAM amplification, corresponding to the reduced asymmetry factors shown in Figure 3.5.

The secondary circulation of mature TCs forms as a way to conserve energy and angular momentum, and the strong cyclonic momentum transport in the low levels is generally counteracted by a strong anticyclonic transport outward from the center aloft (i.e. Figure 2 in Holland 1983b). In weaker TCs, the development of this upper level conserving force is less developed and further disrupted in the presence of shear. It is possible that to compensate this lack of a well-developed exhaust system, the TC remains somewhat asymmetric to conserve momentum through increased frictional (or other) processes. If TCs were to evolve in a perfect environment, some of which has been shown in idealized simulations (i.e. Gopalakrishnan et al. 2011, Bao et al. 2012), it is expected that the vortex will align vertically and momentum flux will mostly be symmetric around the vortex surface center. For the tropical storm cases, movement towards a more symmetric state is noticed, but less of a change upshear confirms that other influences at the weak TC stage are also influencing the momentum transport in such a way as to keep the system somewhat asymmetric. 
3.5.4 Angular Difference Between Shear and Motion and Its Influence on Asymmetric Structure

A remaining question in regards to shear impacts is to what degree (if any) does changing the shear direction in relation to motion affect the low wavenumber surface wind speed asymmetry? Uhlhorn et al. (2014) tried to diagnose this impact but found that the preference for LS asymmetry was prevalent in their four difference groups. Here we present a more detailed analysis of the impacts of shear's relation to motion by first showing the low wavenumber field for each of the $\Delta \theta_{s m}$ groups in Figure 3.7. These fields are presented identically to those in Figure 3.2. All composites display a maximum wavenumber- $0+1$ field oriented on the left side of shear, but clearly the change in $\theta_{s m}$ significantly (at 95\%) impacts the amplitude and phase of the maximum.
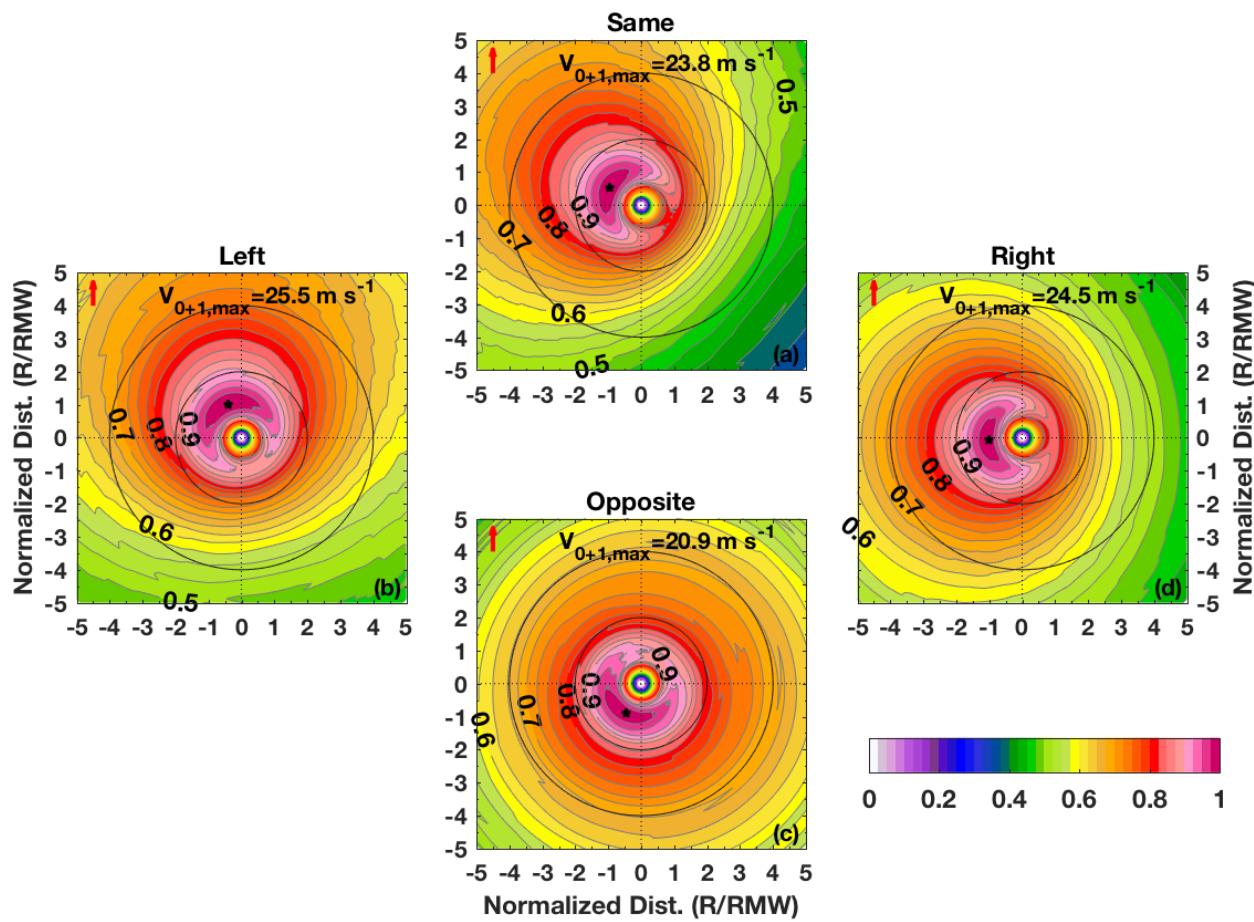

Figure 3.7. Normalized composite analyses plotted similarly to Figure 3.2 but for angle difference bins denoted as (a) same $\left(\left|\Delta \theta_{s m}\right| \leq 22.5^{\circ}\right)$, (b) left $\left(-157.5^{\circ}<\Delta \theta_{s m}<-22.5^{\circ}\right.$ ), (c) opposite $\left(\left|\Delta \theta_{s m}\right| \geq 157.5^{\circ}\right)$, and (d) right $\left(22.5^{\circ}<\Delta \theta_{s m}<157.5^{\circ}\right)$. 
The composite fields displayed in Figure 3.7 indicate that the systematic preference for LS to DS asymmetry as depicted in U14 (and UB11) may not always stand true. Because the scatterometer dataset contains tropical storms and the U14 dataset only includes hurricanes it is possible that weak systems may be influenced more by the difference in the motion and shear. To determine if the strength of the system is suggesting a certain orientation, normalized bivariate PDFs of the wavenumber-1 phase at the maximum amplitude are plotted as a function of $\Delta \theta_{s m}$ in Figure 3.8 for all storms (left panel) and for non-major hurricanes (right panel).
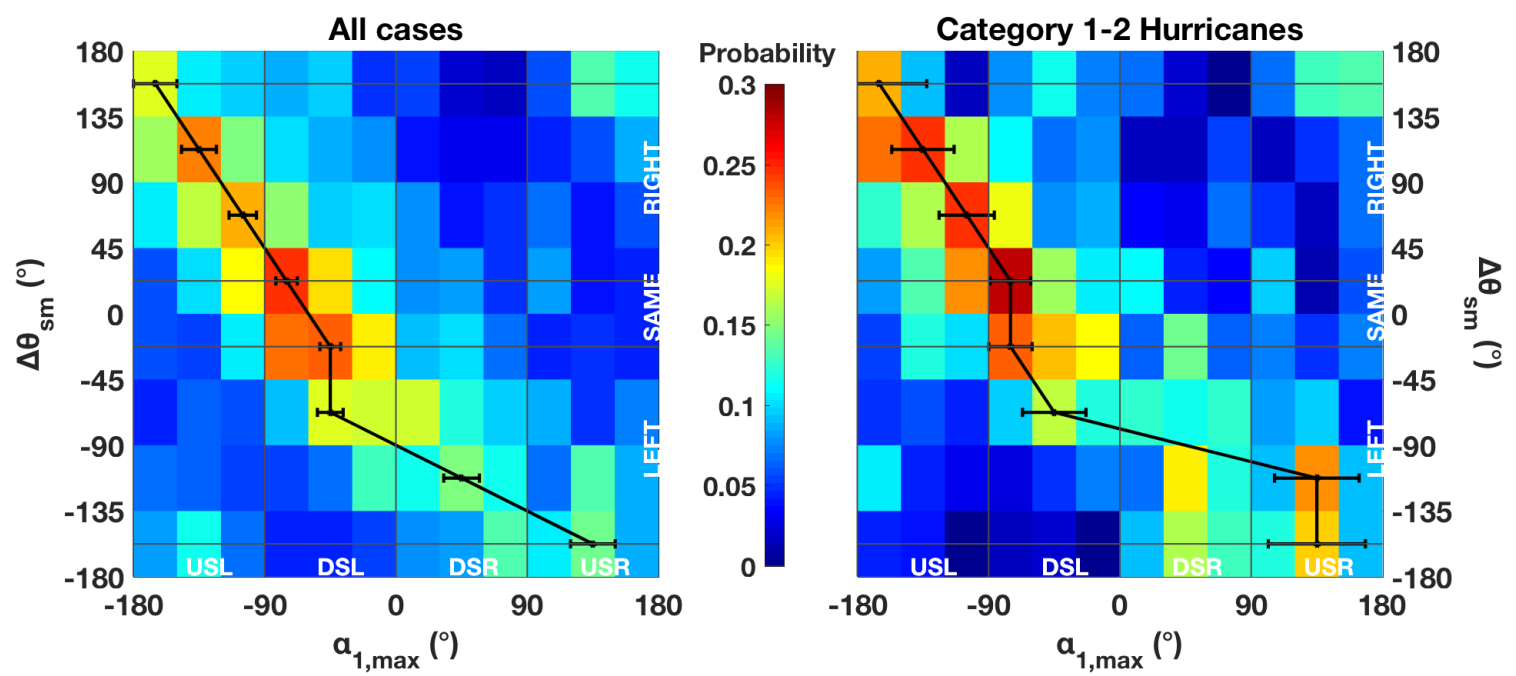

Figure 3.8. In the left panel, a normalized, bivariate PDF for all cases is shown as a function of $\alpha_{1}$, max and of shear-motion angle differences $\left(\Delta \theta_{s m}\right)$. The right panel shows the same type of PDF but only for Category 1-2 hurricanes. The black line indicates the phase bin with the largest probability of occurrence with errorbars indicating the $95 \%$ confidence intervals.

Both joint PDFs indicate a statistically significant (95\%) cyclonic rotation of the low wavenumber asymmetric wind field if moving in order from left, same, right, to opposite. Klotz and Jiang (2016) noticed that after removing the motion vector, the maximum wind speed is oriented to the left of motion in tropical storms and non-major hurricanes. The same can be said for the low wavenumber maxima as a function of $\Delta \theta_{s m}$, 
with the exception of the opposite group compared to the median directions provided in Table 3.2. Note that the directional values in Table 3.2 are Earth-relative. According to Figure 3.8, there is larger amount of variability in the phase of the maximum amplitude that contributes to a disagreement with the other groups.

Table 3.2. Median values for motion and wind shear speed, direction, and direction difference are provided for each $\Delta \theta_{\mathrm{sm}}$ group. Error estimates indicate $95 \%$ confidence intervals.

\begin{tabular}{|c|c|c|c|c|c|}
\hline & $\mathrm{V}_{\text {storm }}\left(\mathrm{m} \mathrm{s}^{-1}\right)$ & $\mathrm{V}_{\text {shr }}\left(\mathrm{m} \mathrm{s}^{-1}\right)$ & $\theta_{\text {storm }}\left(^{\circ}\right)$ & $\theta_{\text {shr }}\left({ }^{\circ}\right)$ & $\Delta \theta_{\text {sm }}\left(^{\circ}\right)$ \\
\hline Same & $5.0 \pm 0.5$ & $6.6 \pm 0.4$ & $-51 \pm 6$ & $-48 \pm 7$ & $0 \pm 1$ \\
\hline Left & $4.0 \pm 0.3$ & $6.0 \pm 0.3$ & $-58 \pm 4$ & $-100 \pm 7$ & $-73 \pm 3$ \\
\hline Opposite & $4.0 \pm 0.5$ & $6.1 \pm 0.7$ & $-62 \pm 8$ & $97 \pm 13$ & $160 \pm 22$ \\
\hline Right & $5.0 \pm 0.3$ & $6.3 \pm-.3$ & $-44 \pm 4$ & $47 \pm 4$ & $78 \pm 2$ \\
\hline
\end{tabular}

Figure 3.9 shows the radial profiles of AIDX for the $\Delta \theta_{s m}$ groups similarly to Figure 3.4. The largest asymmetry values for the full profiles as well as near the RMW are found when vectors point in the same direction while the lowest asymmetry values are found when vectors are oppositely directed, which is in agreement with U14. With the exception of the same compared to the left group (full shear), all profiles are significant at $95 \%$. Note that the same and left groups are significantly more asymmetric beyond the RMW (1-2.5×RMW) compared to the tropical storm cases in Figure 3.4. Considering that all groups have over $50 \%$ of their cases fitting the tropical storm classification and have similar median $V_{\text {storm }}$ and $V_{\text {shr }}$ (Table 3.2), it is evident that the differences in AIDX in Figure 3.9 are a result of the angle differences themselves. When the vectors point in the same direction, the vortex is highly asymmetric. After reducing the shear, the vortex is $\sim 20-25 \%$ more symmetric but remains highly asymmetric compared to the other groups. The opposite group is the most symmetric of the four designations and becomes more 
symmetric beyond $3 \times \mathrm{RMW}$. Also notice that the largest asymmetries occur in the difference between the USL and DSR quadrants, which indicates the USL oriented maximum as in Figure 3.7. The presence of at least moderate shear seems to promote this more symmetric vortex as the low shear conditions are $\sim 15-25 \%$ higher at all radii. Therefore, when vectors are oriented in the same direction or to left of motion, the vortex will be highly asymmetric whereas if they are oppositely oriented, a more symmetric vortex is expected.
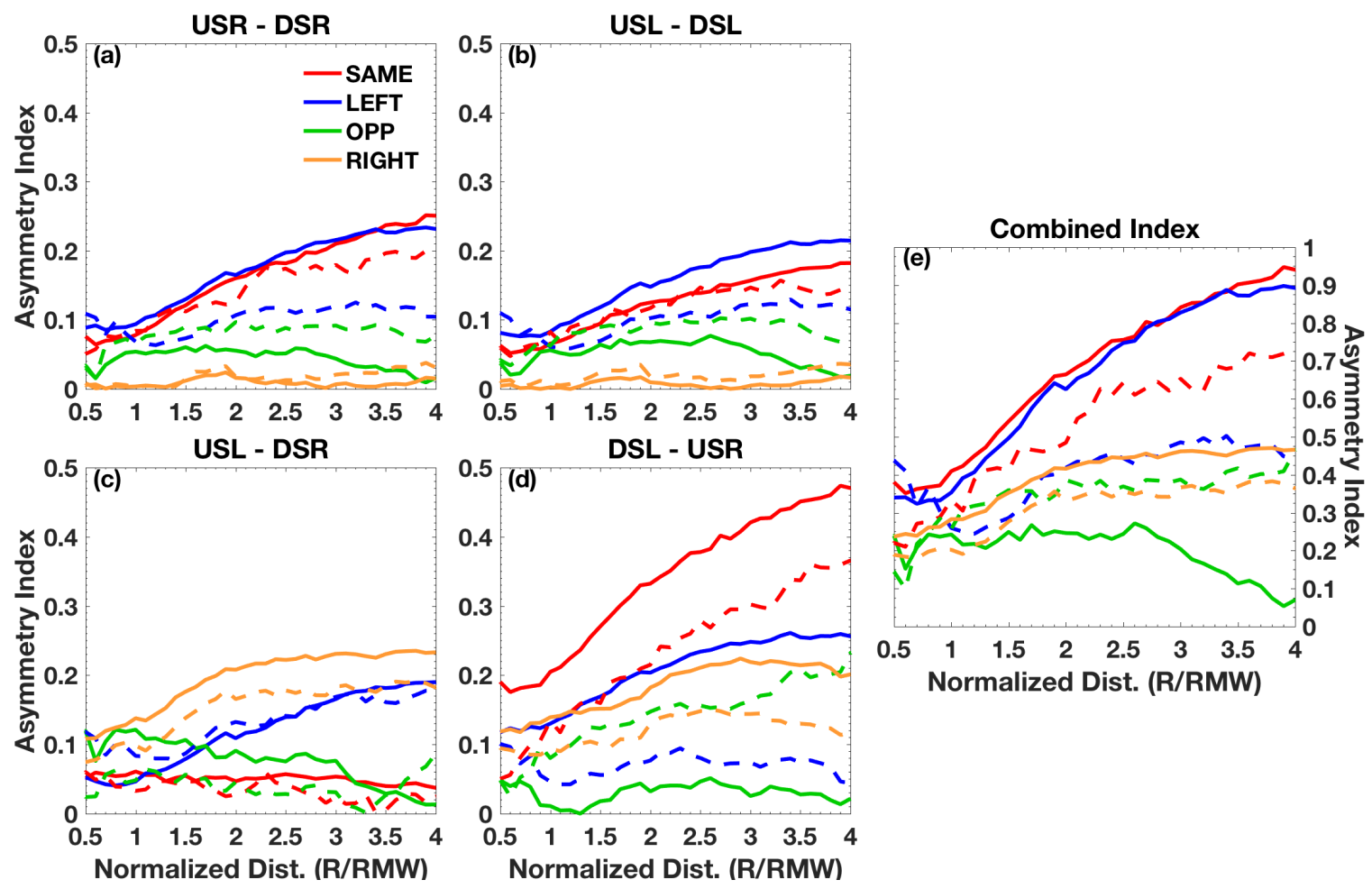

Figure 3.9. As in Figure 3.4 but for the $\Delta \theta_{s m}$ groups.

Using the quadrant based AAM flux terminology, as before, it is possible to see how the angular momentum transport is influencing the $\Delta \theta_{s m}$ relative low wavenumber analysis. Figure 3.10 provides the shear-relative quadrant profiles of AAM flux in a similar manner to Fig 3.5. The same and right groups, left group, and opposite group exhibit their most prominent AAM flux in the DSL, DSR, and DSL/USL quadrants, 
respectively. Compared to the orientation of the low wavenumber fields (Figure 3.7), there is an apparent downwind rotation from the location of the maximum AAM flux. For example, the same group has a strong AAM flux in both DS quadrants with apparent weak outward flux in the USL quadrant. The strong inward flux DS occurs upwind of the low-wavenumber maximum. On the other hand, the opposite group exhibits a maximum momentum transport DSL (with some large values USL), in accordance with the downwind location of the maximum phase relative to the maximum AAM flux. While the increased variability within this stratification could be contributing to this difference, the authors suggest that a possible reason for this difference in orientation is the more symmetric structure of the vortex.
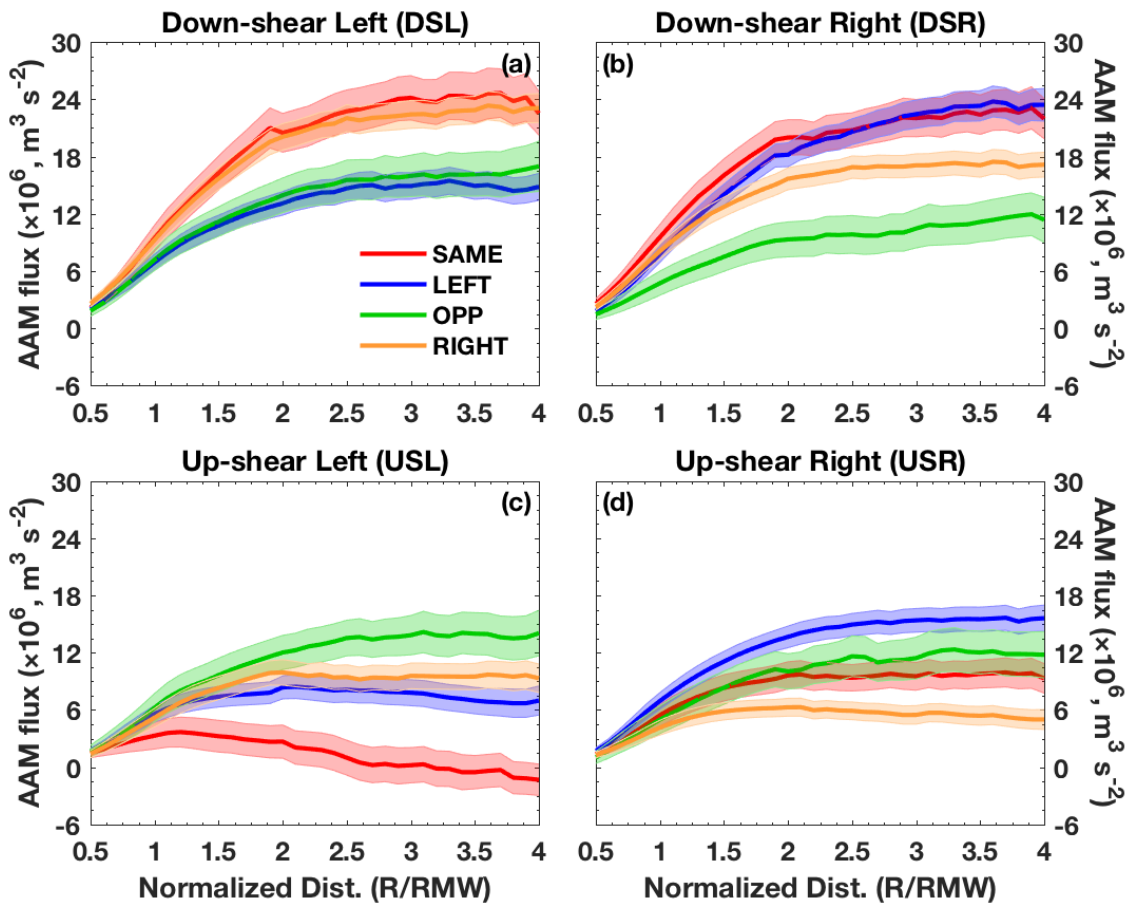

Figure 3.10. As in Figure 3.5 but for the $\Delta \theta_{s m}$ groups.

The AAMF description above is a reasonable explanation of the rotating asymmetry with changing $\theta_{s m}$ groups, but it is complicated by several factors. Thomsen et 
al. (2015) conclude that as the storm speed increases, the vertical velocity moves from a more symmetric state to asymmetric state with the largest asymmetry down-motion-left (DML), which translates to a DM to DMR wind asymmetry (motion not removed). At translation speeds near the mean observed for the scatterometer cases, the vertical velocity asymmetry peak occurs DML but with more variability. As was indicated by $\mathrm{KJ} 16$, after removing the motion vector, the asymmetric structure rotates DM to LM as a function of TC intensity. This orientation would occur underneath or slightly downwind of the vertical motion asymmetry. In the figures shown here, all but the opposite case exhibit the LM to DML wavenumber-1 asymmetry (based on the shear-rotated values in Table 3.2), so it is still in agreement with the expectations of Shapiro (1983) and Thomsen et al. (2015).

One more complication is the lack of consideration of a locally induced shear or imposing flow at low levels. A presentation by Kaplan et al. (2014) showed through comparison with airborne Doppler radar data, a local shear analyzed in the GFS (Global Forecast System) model field is somewhat similar to those determined by the radar. However, they note there is a significant case-by-case variability in the model estimate of local shear. Reasor et al. (2013) stipulates that the local storm induced wind shear is generally to the right of the environmental shear, producing a low level flow across the vortex from DSR to USL. These authors also show that the maximum vertical velocity is oriented DS (with some variability in the DSR direction), which supports a convective maximum downwind (or DSL) as has been shown in other convective asymmetry studies (C06; Wingo and Cecil 2010; Hence and Houze 2011; Jiang and Ramirez 2013; Zagrodnik and Jiang 2014; Tao and Jiang 2015). The convective asymmetry is likely 
going to have an influence on the surface wind structure as well (U14; UK09) but generally developing a LS to DSL maximum asymmetry. It is possible that for oppositely pointing shear and motion vectors, the super-imposed low-level flow along with the convective maximum pose an alternative setup compared to the result of Reasor et al. (2013) from above, where a convective maximum occurs more to the left of the environment shear value. Increased angular momentum transport would then form DSL and promote a wind speed asymmetry USL. Clearly, the processes involved necessitate more investigation and inter-comparison but this task is beyond the scope of the current presentation.

\subsection{Summary and Conclusions}

In the current study, a large dataset of neural network corrected scatterometer surface winds is utilized to determine detailed information about the low wavenumber asymmetric surface wind structure and to quantify the vertical wind shear influence on this structure. Several previous studies using aircraft data (Uhlhorn and Rogers 2008; U14) and satellite data (UK09; UB11) provide insight into the expected relationships between wind shear and storm motion and their relative impact on the surface wind asymmetry. However, these studies are limited in terms of the scope of their dataset or which types of TCs are included in their analyses. While inherent with several drawbacks, the global scatterometer dataset provides an avenue for obtaining statistically sound results for various stratifications in the low wavenumber, Fourier decomposition framework discussed in V14 and U14.

At the beginning of this study, the authors sought to answer several important questions regarding applicability of the data and methods used as well as diagnosing and 
quantifying the general structure and asymmetry of surface wind speed in the presence of shear. On the basis of the results of the composite analyses and discussion of mechanisms for the various differences in the low wavenumber asymmetry, the following main conclusions provide answers to those questions:

- Through validation with SFMR data, corrected scatterometer winds in association with the analysis method are reliable for diagnosing low wavenumber asymmetric surface wind structure in TCs, which is a foundation for robust statistical examination of the surface wind structure in TCs.

- Examination of the asymmetry index compared to cases that experience weak wind shear reveals that tropical storms are the most asymmetric of the TC intensity groups near the RMW but that all intensity groups are statistically significantly impacted by shear outside the RMW. Absolute angular momentum flux profiles suggest that an infusion of higher momentum occurs DS such that an increase of inward momentum flux is found upwind of the surface wind asymmetry maximum. Once shear is lowered, this momentum flux becomes more evenly distributed around the cyclone and reduces the asymmetry of the surface wind field, especially noticeable in the hurricane cases.

- When changing the shear in relation to motion from left, same, right, or opposite, the orientation of the maximum asymmetry (near the RMW) rotates cyclonically from DS, DSL, LS, to USL, respectively. The order of these groups is also in general order of the magnitude of their asymmetry, where same and left groups (opposite) are highly asymmetric (symmetric) in the presence of shear. Absolute angular momentum flux converges DSL to LS instead of DSR to DSL for the opposite $\Delta \theta_{s m}$ 
cases, further confirming that the maximum wavenumber asymmetry occurs downwind of the largest AAM flux.

The results discussed provide a detailed examination of the impacts of shear on the surface wind field and how the asymmetric structure changes with storm intensity and other factors. The idea of importation or increase of inward transport of absolute angular momentum upwind of the low wavenumber maximum suggests a coincident occurrence with the development of upward motion (UK09; Hence and Houze 2011; Reasor et al. 2013). The suggestion of a superimposed low-level uniform flow due to locally induced wind shear seems to be an avenue down which a connection between convection/precipitation and winds can be established. A companion study (part II) related to this work discusses the change in surface wind structure as it pertains to intensity change, which could have implications for improved forecasting of intensity change events. 


\section{EXAMINATION OF SURFACE WIND ASYMMETRIES IN TROPICAL}

\section{CYCLONES: PART II. INTENSITY CHANGE}

\subsection{Abstract}

Low wavenumber surface wind structure analyses derived from global raincorrected scatterometer winds are used to determine structure differences associated with various types of intensity change in a shear-relative framework. A presentation of the quantified asymmetry in rapidly intensifying, slowly intensifying, steady state, and weakening tropical cyclones indicates that the low wavenumber response is more closely related to the initial intensity rather than a future 24-hour intensity change. Absolute angular momentum flux analyses also tend to follow results that are associated with initial intensity. When comparing an initial against an established stage of rapid intensification, it is confirmed that tropical cyclone asymmetry is reduced significantly in the established stage but non-trivial values remain at outer portions of the vortex due to moderate wind shear. In the framework of a composite rapid intensification event, the wavenumber-1 amplitude contribution is symmetrized near the maximum wind speed while the phase rotates from downshear-left at the event initiation to an upshear-left orientation. After comparing this changing low wavenumber surface wind structure to a coincident precipitation dataset, it is confirmed that early in the event progression, the maximum rain parameter is located radially equivalent but upwind of the low wavenumber maximum amplitude. In the context of the upwind absolute angular momentum flux, this suggests that the low wavenumber asymmetry structure is likely a result of rather than initiator of precipitation and thermodynamic processes. 


\subsection{Introduction}

Depending on how internal vortex-specific and environmental variables change as a TC progresses, there is a spectrum of intensity and intensity change outcomes, ranging from rapid intensification (RI) to weakening. Previous environmental studies have examined effects of relative humidity, instability, divergence, and background vorticity in order to develop a better understanding their impacts on intensification (Kaplan and DeMaria 2003; Hendricks et al. 2010; Kaplan et al. 2010). One particular result from these works is the general consensus that intensifiers, especially ones experiencing RI, have more favorable environments (i.e., lower wind shear and high SSTs) than TCs that remain at a steady state or weaken. The intensity of an existing disturbance also plays a role as most cases that experience intensification and RI initiate as tropical storms or nonmajor hurricanes (Kaplan and DeMaria 2003; Kieper and Jiang 2012). Knowing that TCrelated damage increases exponentially with intensity [Florida Commission on Hurricane Loss Projection (FCHLP), 2013] and that coastal communities are growing in population (Pielke et al. 2008), the risk for increased damage is compounded by the uncertainty in the processes involved with intensity change.

Intensification of TCs, especially for RI $\left(\Delta V_{\max } \geq 30 \mathrm{kt}\right.$ over 24 hours, where $1 \mathrm{kt}$ $=0.514 \mathrm{~m} \mathrm{~s}^{-1}$ ) has recently received much attention, with a focus of predicting it with more accuracy through diagnosing specific dynamical, thermodynamical, and precipitative features. Sanger et al. (2014) notes that intensification theories have evolved over the past several decades. The theories mentioned in Chapter 1 summarily include: (a) intensity increases due to convectively induced and conserved absolute angular momentum above the boundary layer (CISK, Charney and Eliasen 1964; Ooyama 1969; 
Carrier 1971, Schubert and Hack 1982), (b) ideas related to a positive feedback loop involving near-surface wind speed and evaporation from the ocean as related to thermodynamic disequilibrium (WISHE, Rotunno and Emanuel 1987), and (c) recent ideas related to observations suggesting that vortical convective structures induce radial inflow above the BL (and convergence of momentum). The radial pressure gradient increases with the inflow (Nguyen et al. 2008; Montgomery et al. 2009, 2014; Smith et al. 2009; Smith and Montgomery 2015) and suggests that the maximum tangential wind speed occurs within the boundary layer.

There is evidence to suggest that the VHTs or convective bursts (CBs) near the radius of maximum wind (RMW) are at least associated with RI (Hendricks et al. 2004; Guimond et al. 2010; Nguyen et al. 2011; Chen and Zhang 2013; Rogers et al. 2015; Chen and Gopalakrishnan 2015; Rogers et al. 2016), but it is still not clear what role they serve in the intensification process. Nolan et al. (2007) agrees with the above studies and indicates through a numerical simulation that the kinetic energy efficiency is significantly higher when convection (i.e latent heating) is located closer to the storm center. This result further agrees with earlier work from Schubert and Hack (1982) and Shapiro and Willoughby (1982) in regards to the location of a heat or momentum source near the TC center, which impacts vortex intensity by increasing or decreasing inertial stability.

Several recent studies (Kieper and Jiang 2012; Zagrodnik and Jiang 2014; SuscaLopata et al. 2015; and Tao and Jiang 2015) have examined the RI problem through use of satellite precipitation data. These studies do not necessarily contradict the theory that VHTs or CBs are involved in the initiation of RI but stress the importance of the entire precipitation field. Jiang (2012) notes that VHTs are not necessarily a sufficient condition 
to initiate RI. All of these works suggest that the contribution of total rainfall, mainly from moderate and shallow precipitation, is of vital importance in initiating and maintaining RI. They also suggest a move toward increased symmetry of the precipitation field after RI starts and notice a significant change in the upshear and upshear-right quadrants during the progression of RI. Zawislak et al. (2016) further confirms this symmetrization from the perspective of shear-relative thermodynamic information by suggesting that a humidification upshear is needed for RI to occur. This result also agrees with studies that indicate the cyclonic progression of convection rotates from downshear-left to upshear quadrants as storms intensify (i.e., Black et al. 2002; Reasor et al. 2009). Symmetry associated with maturing TCs is not a new concept in terms of kinematics (Willoughby 1990; Kossin and Eastin 2001; Kossin et al. 2007; Nguyen et al. 2011, Klotz and Jiang 2017 [Chapter 3]), but a relatable precipitation symmetry is quantifiable using the methods in these studies.

TC intensification is clearly a complicated process that involves multiple changes in the dynamic, thermodynamic, and precipitation fields. Reasor et al. (2009) notes that processes related to both axisymmetric and asymmetric phases of intensification play a significant role in overall TC intensification. Much of the recent attention for intensity change has focused on precipitation/convection, and there seems to be a lack of evaluation of the changes in surface wind features as it relates to the TC intensification spectrum. Understanding changes in surface wind structure is important from multiple perspectives. On the forecasting side, operational centers worldwide use a maximum sustained surface wind speed as their official TC intensity metric (Jarvinen et al. 1984; Landsea and Franklin 2013), and on the research side, surface wind interaction with the 
sea surface has implications for future intensification (Cione and Uhlhorn 2003; Cione et al 2013) and is related to processes occurring higher in the boundary layer.

The maximum wind speed $\left(V_{\max }\right)$ is a quantity that is difficult to accurately obtain without the assistance of aircraft reconnaissance, primarily from SFMR (Uhlhorn et al. 2007; Klotz and Uhlhorn 2014) and GPS dropsonde surface winds (Hock and Franklin 1999; Franklin et al. 2003). As a result, many forecasts are heavily reliant on estimates of TC intensity derived from satellite products because of the lack of regularly obtained instorm aircraft data (Rappaport et al. 2009). Wind speed products derived from satellitebased instruments, such as scatterometers (Draper and Long, 2002, 2004; Figa-Saldaña et al. 2002; Hoffman and Leidner 2005) as noted previously are useful for diagnosing TC size (Chavas and Emanuel 2010; Chan and Chan 2012; Chavas et al. 2016), but the nominal horizontal resolution radially outward from the TC center makes it more difficult to determine small scale wind features, causing underestimates of maximum wind speeds in hurricanes. Following a methodology similar to Uhlhorn and Nolan (2012), an ongoing study (Nolan and Klotz 2016) is assessing the underestimate through simulated scatterometer winds to quantify a 'best case' scenario from the nominal wind observations. Complications due to interference from rain also hinder accurate wind speed retrievals near the RMW. As an expansion on the results of U14, Chapters 2 and 3 show that low wavenumber evaluations of asymmetric structure are meaningful after applying a correction to the original winds (Stiles et al. 2014) and describe that the surface wind asymmetry is significantly impacted by wind shear both as a function of TC intensity and of the wind shear directional relation to storm motion. Further evaluation of the shear-relative absolute angular momentum (AAM) transport revealed that in the 
presence of shear, AAM converges downshear-right and upwind of the maximum surface asymmetry.

To date, only case studies of surface wind fields in intensifying TCs (Molinari and Skubis 1985; Rogers and Uhlhorn 2008; Molinari and Vollaro 2010; Uhlhorn et al. 2014) have provided an idea of the change in certain surface features. Therefore, as a follow-up to Klotz and Jiang (2017, herein referred to as KJ17 [Chapter 3 results]) and further expansion of the principles in Uhlhorn et al. (2014), the current study seeks to comprehensively and statistically evaluate the change in surface wind asymmetry as a function of intensity change from a large dataset of scatterometer surface winds. Similarly to KJ17, composite analyses are produced for intensity change stratifications (Jiang and Ramirez 2013; Zagrodnik and Jiang 2014) and an evaluation of the factors influencing the structure differences is provided. Additionally from the perspective of various periods of an RI event, a glimpse at the progression of the low wavenumber asymmetry compared to precipitation features determined from the Tropical Rainfall Measuring Mission (TRMM) Microwave Imager (TMI) is described. This chapter is separated into several sections, including a description of the data and a summary of the methodology in section 4.3. A third section describes the results from the perspective of the different intensification groups and for the changes as a function of time relative to the onset of RI. The final section describes the conclusions and next steps.

\subsection{Data and Methodology}

A detailed description of the data and analysis methods is provided in KJ16 (Chapter 2) and KJ17 (Chapter 3), and the authors direct any questions regarding their 
specifics to that reference. However, it is necessary to describe the pertinence of the scatterometer data to a study involving TC intensification.

\subsubsection{Summary of Scatterometer Data and Intensity Metrics}

As a reminder, the scatterometer data used in this study were processed with a horizontal resolution of $12.5 \mathrm{~km}$, and a neural network correction (Stiles et al. 2014) helps address a rain contamination issue for wind speeds at C-band frequency. All scatterometer wind speeds used herein will have the NN correction applied. As noted in Chapters 2 and 3, only cases with at least $70 \%$ coverage within $250 \mathrm{~km}$ of the TC center (and $100 \%$ within $125 \mathrm{~km}$ ) are used in this study.

Track and intensity are obtained from the hurricane best track data (Landsea and Franklin 2013) for the North Atlantic and Eastern North Pacific basins. JTWC storm files provide best track information for the other TC-prone basins. From these data, center position, storm motion direction and speed, and $V_{\max }$ are provided every six hours. Vertical wind shear data are obtained from the Statistical Hurricane Intensity Prediction Scheme (SHIPS) database (DeMaria and Kaplan 1994, 1999) for the same periods as the best track data. A deep layer wind shear is calculated between $850 \mathrm{mb}$ and $200 \mathrm{mb}$ with the TC vortex removed up to $500 \mathrm{~km}$ from the center.

\subsubsection{TRMM Microwave Imager (TMI) Data}

While the TMI is not necessarily the most accurate or precise measure of rain rate (Lonfat et al. 2004; Nesbitt et al. 2004; Viltard et al. 2006), it compares well to the Precipitation Radar (PR) in terms of detecting rain and determining raining area. For the purposes of the present study, it is one of the authors' goals to provide a general idea of how the low wavenumber asymmetry relates to the precipitation field. TC-centered $2 \mathrm{~A} 12$ 
rain rate (Kummerow et al. 2001) fields are gridded at $10 \mathrm{~km}$ horizontal resolution and extend out to a distance of $300 \mathrm{~km}$. From the gridded fields, radial location and shearrelative azimuth of the maximum rain rate $(\mathrm{RR})$ are calculated. Total raining area for all $\left(\mathrm{RR} \geq 0.5 \mathrm{~mm} \mathrm{~h}^{-1}\right)$, light $\left(0.5 \leq \mathrm{RR}<5 \mathrm{~mm} \mathrm{~h}^{-1}\right)$, moderate $\left(5 \leq \mathrm{RR}<10 \mathrm{~mm} \mathrm{~h}^{-1}\right)$, and heavy $\left(\mathrm{RR} \geq 10 \mathrm{~mm} \mathrm{~h}^{-1}\right)$ precipitation is determined for the entire grid as well as for each shear-relative quadrant. These same values are also calculated for radial distances from the TC-center to obtain radial profiles of raining area.

\subsubsection{Methods Specific to Intensity Change Analysis}

KJ17 provide evidence that using a low wavenumber asymmetry analysis is not only useful but also important for diagnosing changes in the asymmetric structure of the surface winds. Following their equations, the same concepts are presented here in an intensity change framework. Because KJ16 and KJ17 indicate that wind shear has a significant influence on the surface wind structure, composite analyses are produced on a shear-relative grid (with storm motion removed). Jiang and Ramirez (2013) and Zagrodnik and Jiang (2014) describe in detail the classification techniques for satellite overpasses within the TC intensification framework. They determine periods of intensification or weakening based on the best track $V_{\max }$ at the time of the overpass compared to the future 24-hour $V_{\max }$. RI cases are classified by a $\Delta V_{\max }$ of at least $30 \mathrm{kt}$, while slowly intensifying (SI) cases are classified by $\Delta V_{\max }$ of between 10 and $30 \mathrm{kt}$. Steady state [SS, or neutral in Jiang and Ramirez (2013)] cases change by less than $10 \mathrm{kt}$ over a 24 hour period while weakening (WK) cases decrease by more than $10 \mathrm{kt}$ in 24 hours. 
In addition to these four stratifications, the RI cases are further separated into RIInitial and RI-Continuing as described in Zagrodnik and Jiang (2014). The purpose of this classification is to determine features within the first 12 hours of an RI event and how they might differ from features that occur later in the intensification process. Any differences found are expected to be indicative of RI initiation and have potential to improve predictability. Table 4.1 provides the basin statistics for each of the above stratifications, with specific mention of RI-Initial (RII) versus RI-Continuing (RIC) as well as intensifying (INTS) versus non-intensifying (NINTS) cases. INTS cases comprise both RI and SI classifications while NINTS contain SS and WK classifications. Note that a little more than one third of the cases are considered intensifying and about one quarter of those cases (10\% of the overall sample) are classified as RI. Figure 1 provides a map of the various geographical locations of the RI associated scatterometer data, specifically indicating the RII and RIC cases.

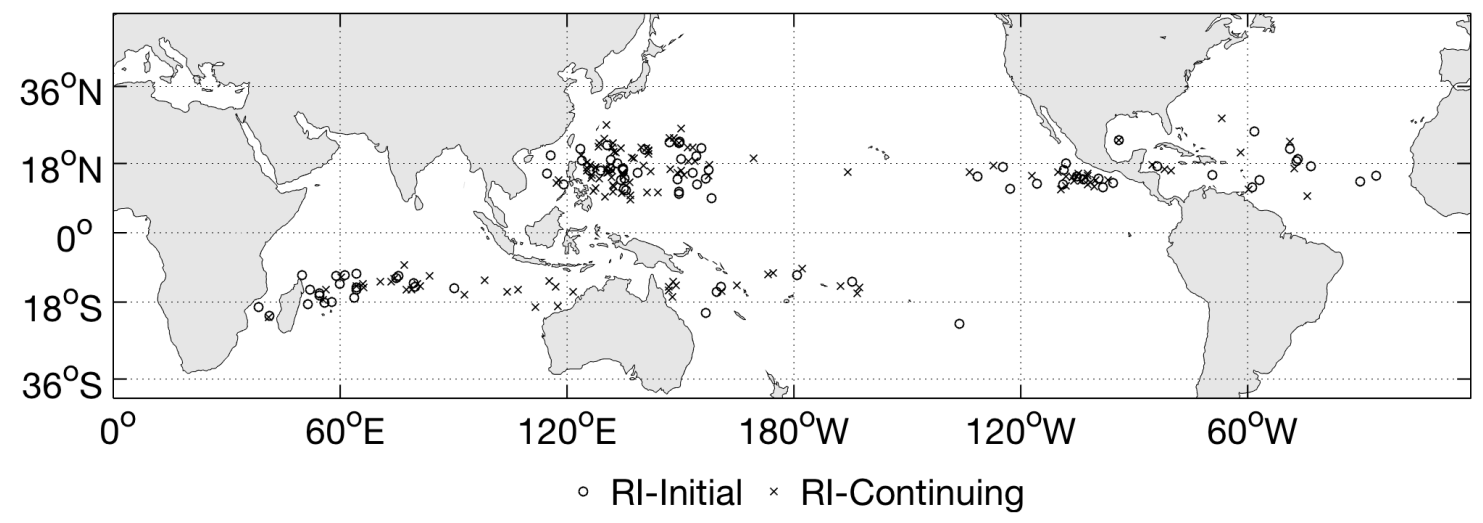

Figure 4.1. Geographical locations of the scatterometer are provided for RI cases with RIInitial and RI-Continuing explicitly marked. 
Table 4.1. The number of cases for each intensity change classification (RI, SI, SS, and WK) is provided as a function of TC basin. Additional indicators for RII and RIC cases as well as for intensifying and non-intensifying (INTS vs. NINTS) cases are provided too. Values in parentheses represent the percentage first within the respective intensity change group followed by the percentage within the basin. For the INTS and NINTS labels, values in parentheses indicate the number and percentage of the total sample.

\begin{tabular}{|c|c|c|c|c|c|c|c|}
\hline & & & ALL & NATL & ЕРСР & WPAC & SHEM \\
\hline \multirow{4}{*}{ 究 } & RI & & 219 & $\begin{array}{c}22 \\
(10.0 \% \\
4.4 \%)\end{array}$ & $\begin{array}{c}33 \\
(15.1 \% \text {, } \\
6.2 \%)\end{array}$ & $\begin{array}{c}99 \\
(45.2 \%, \\
17.3 \%)\end{array}$ & $\begin{array}{c}65 \\
(29.7 \%, \\
10.5 \%)\end{array}$ \\
\hline & & RII & 85 & $\begin{array}{c}12 \\
(14.1 \% \\
2.4 \%)\end{array}$ & $\begin{array}{c}12 \\
(14.1 \% \text {, } \\
2.3 \%)\end{array}$ & $\begin{array}{c}34 \\
(40.0 \% \\
6.0 \%)\end{array}$ & $\begin{array}{c}27 \\
(31.8 \% \text {, } \\
4.3 \%)\end{array}$ \\
\hline & & RIC & 134 & $\begin{array}{c}10 \\
(7.5 \%, 2.0 \%)\end{array}$ & $\begin{array}{c}21 \\
(15.7 \% \text {, } \\
3.9 \%)\end{array}$ & $\begin{array}{c}65 \\
(48.5 \% \\
11.4 \%)\end{array}$ & $\begin{array}{c}38 \\
(28.4 \%, \\
6.1 \%)\end{array}$ \\
\hline & SI & & 593 & $\begin{array}{c}133 \\
(22.4 \% \\
26.9 \%) \\
\end{array}$ & $\begin{array}{c}96 \\
(16.2 \%, \\
18.1 \%) \\
\end{array}$ & $\begin{array}{c}180 \\
(30.4 \%, \\
31.5 \%)\end{array}$ & $\begin{array}{c}184 \\
(31.0 \% \text {, } \\
29.6 \%) \\
\end{array}$ \\
\hline \multirow{2}{*}{ 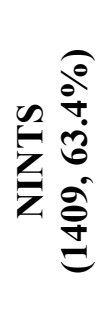 } & SS & & 758 & $\begin{array}{c}210 \\
(27.7 \% \\
42.4 \%)\end{array}$ & $\begin{array}{c}207 \\
(27.3 \%, \\
38.8 \%)\end{array}$ & $\begin{array}{c}131 \\
(17.3 \% \\
22.9 \%)\end{array}$ & $\begin{array}{c}210 \\
(27.7 \%, \\
33.8 \%)\end{array}$ \\
\hline & WK & & 651 & $\begin{array}{c}130 \\
(20.0 \% \\
26.3 \%)\end{array}$ & $\begin{array}{c}197 \\
(30.3 \%, \\
37.0 \%)\end{array}$ & $\begin{array}{c}161 \\
(24.7 \% \\
28.2 \%)\end{array}$ & $\begin{array}{c}163 \\
(25.0 \% \text {, } \\
26.2 \%)\end{array}$ \\
\hline 胥 & & & 2221 & 495 & 533 & 571 & 622 \\
\hline
\end{tabular}

Something to consider in relation to the intensity change composites is differences in storm size, shear and storm motion speed, and their angular difference. KJ17 and U14 indicate that a statistically significant clockwise (counter-clockwise) rotation of the motion- (shear-) relative wavenumber-1 phase occurs with increasing storm (shear) speed, especially for tropical storms and non-major hurricanes. After removing motion impacts, the wind shear has a more dominant impact on the surface wind asymmetry. Figure 4.2 provides stacked histograms of RMW, wind shear speed $\left(V_{s h r}\right)$, storm motion 
speed $\left(V_{\text {storm }}\right)$, and shear - motion angle difference $\left(\Delta \theta_{s m}=\theta_{s h r}-\theta_{s t o r m}\right)$ for the four intensity change groups above (RI, SI, SS, and WK). The values shown are percentages associated with the individual groups. In many instances, there is not a significant difference between the conditions experienced by INTS or NINTS tropical cyclones. The text values above each bar, which are the percentage of INTS cases, indicate that $V_{\text {shr }}$ tends to be lower for INTS and that shear more often than not is to left of the motion vector.

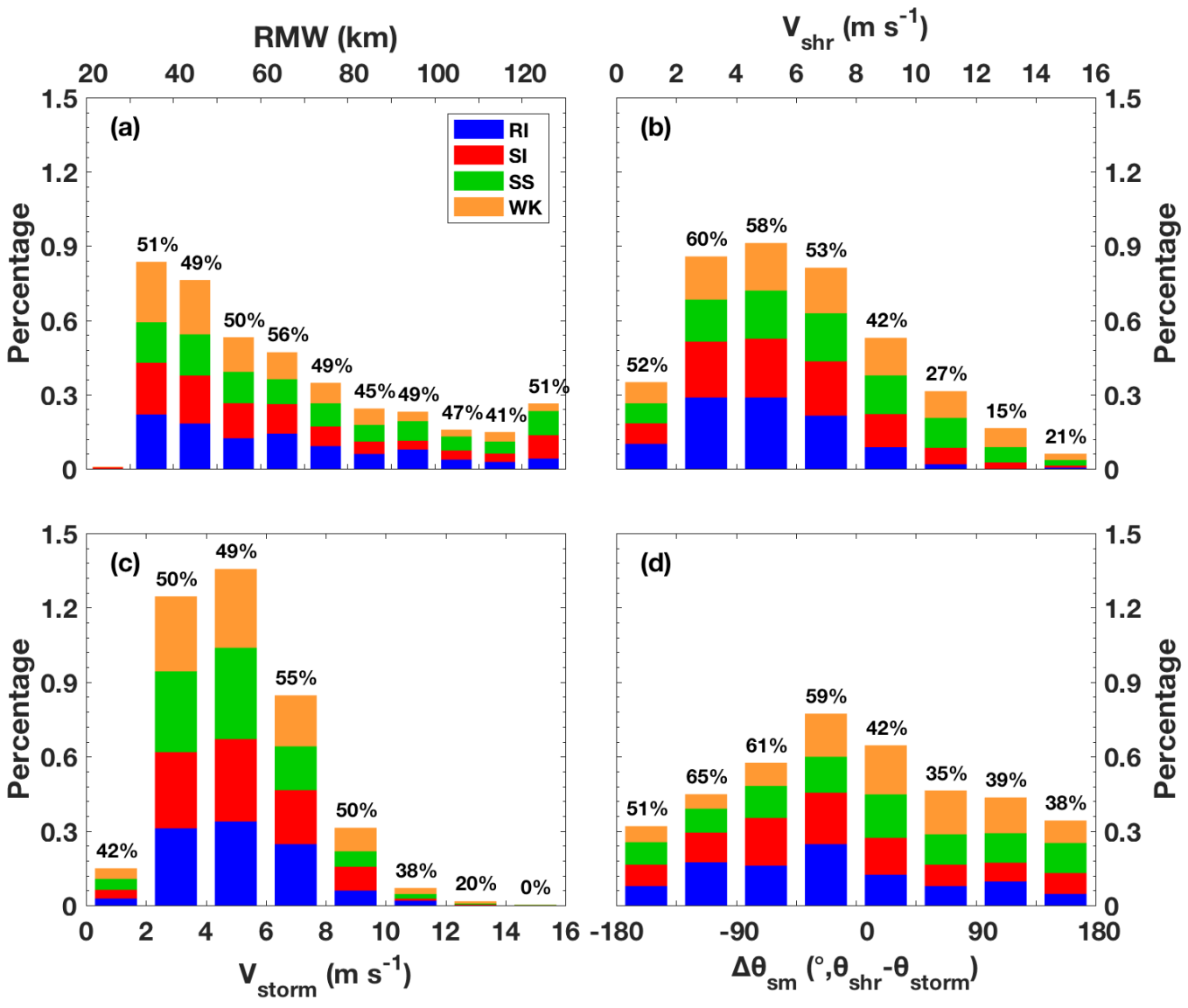

Figure 4.2. Stacked histograms of (a) RMW, (b) wind shear speed $\left(V_{\text {shr }}\right)$, (c) storm motion speed $\left(V_{\text {storm }}\right)$, and (d) shear and motion angle difference $\left(\Delta \theta_{s m}\right)$ are shown for the four intensity change groups (RI, SI, SS, and WK). Values plotted are given as a percentage relative to the total within a particular intensity change group. The text above each bar indicates the percentage of INTS cases within the respective variable bin.

These stratifications are beneficial for noticing significant differences between the various types of intensity change. Another way to view significant differences in the 
surface wind speed is through examination of a temporally defined RI event. Every event has a pre-event period, onset of RI, continuation of RI, an ending period, and a post-event period. Tao and Jiang (2015) define a timeline over which convective and precipitation features are expected, and their time designations are used in the current study to determine changes in surface wind structure as it relates to the various stages of an RI event. For the purposes here, times are considered relative to the onset of $\mathrm{RI}\left(\mathrm{RI}_{0}\right)$, where periods 12-24 hours and 0-12 hours prior to RI are considered the pre-event period ( $\mathrm{RI}_{0^{-}}$ $24 \leq t<\mathrm{RI}_{0}-12$ and $\mathrm{RI}_{0}-12 \leq t<\mathrm{RI}_{0}$, respectively). Any time within 0-12 hours after the start of an RI period (i.e. the first time where an increase of at least $30 \mathrm{kt}$ occurs) is considered the onset period $\left(\mathrm{RI}_{0} \leq t<\mathrm{RI}_{0}+12\right)$, and the continuing period occurs until the last $30+$ kt 24 hour wind increase $\left(\mathrm{RI}_{0}+12 \leq t<\mathrm{RI}_{\mathrm{E}}-24\right.$, where $\mathrm{RI}_{\mathrm{E}}$ marks the end of the RI event). This time is considered the start of the RI ending period $\left(\mathrm{RI}_{\mathrm{E}}-24<t \leq \mathrm{RI}_{\mathrm{E}}\right)$, followed by a final 12 hour period that occurs after the event has finished $\left(\mathrm{RI}_{\mathrm{E}}<t \leq \mathrm{RI}_{\mathrm{E}}\right.$ +12 ). Note that the variable $t$ is the scatterometer time. Table 4.2 provides the number of cases per each of these periods but without separation by basin. Figure 4.3 provides stacked histograms as in Figure 4.2 but for the RI event time periods. For times prior to or within the RI onset (text percentages in Figure 4.3), a higher occurrence of large RMW TCs is found along with a preference for low to moderate wind shear and left or oppositely pointing shear vectors relative to motion. There is not a strong preference for storm speed for these RI periods. 
Table 4.2. The number of cases associated with each RI event period is provided along with the percent contribution to the sample size. Nomenclature in the period labels are $t$ for the respective time, $\mathrm{RI}_{0}$ for the time marking the start of $\mathrm{RI}$, and $\mathrm{RI}_{\mathrm{E}}$ for the time marking the end of the RI event.

\begin{tabular}{|c|c|c|}
\hline & Count & Percent of total \\
\hline $\mathrm{RI}_{0}-24 \leq \mathrm{t}<\mathrm{RI}_{0}-12$ & 29 & $5.0 \%$ \\
\hline $\mathrm{RI}_{0}-12 \leq \mathrm{t}<\mathrm{RI}_{0}$ & 50 & $8.7 \%$ \\
\hline $\mathrm{RI}_{0} \leq \mathrm{t}<\mathrm{RI}_{0}+12$ & 90 & $15.7 \%$ \\
\hline $\mathrm{RI}_{0}+12 \leq \mathrm{t}<\mathrm{RI}_{\mathrm{E}}-24$ & 101 & $17.6 \%$ \\
\hline $\mathrm{RI}_{\mathrm{E}}-24<\mathrm{t} \leq \mathrm{RI}_{\mathrm{E}}$ & 226 & $39.3 \%$ \\
\hline $\mathrm{RI}_{\mathrm{E}}<\mathrm{t} \leq \mathrm{RI}_{\mathrm{E}}+12$ & 79 & $13.7 \%$ \\
\hline
\end{tabular}
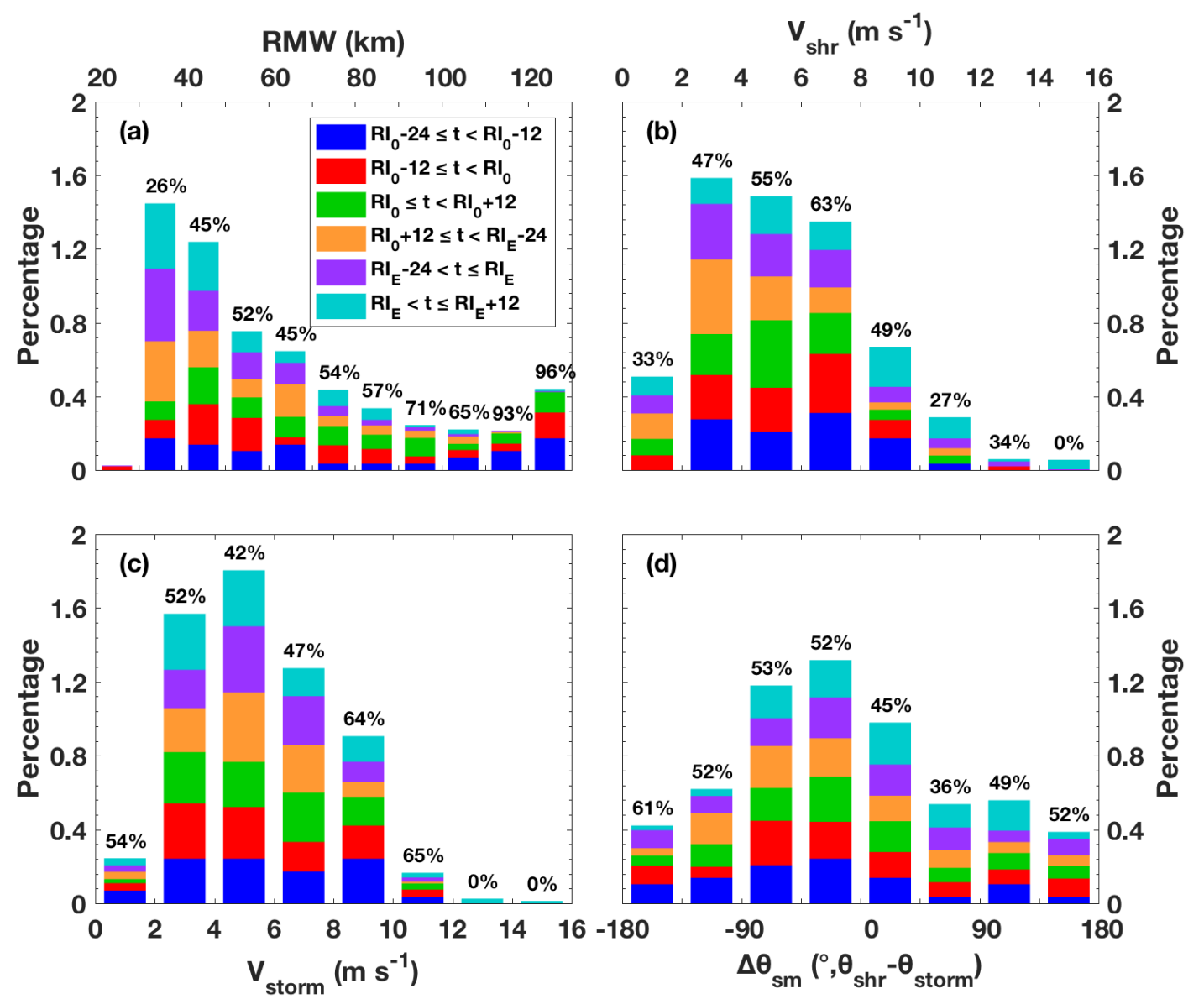

Figure 4.3. Similar to Figure 4.2 but for RI event periods as defined by the RI start $\left(\mathrm{RI}_{0}\right)$ and end $\left(\mathrm{RI}_{\mathrm{E}}\right)$ times. The symbol $t$ refers to the scatterometer time relative to $\mathrm{RI}_{0}$ or $\mathrm{RI}_{\mathrm{E}}$. The text above each bar is the percentage of cases that occur prior to or during the first 12 hours of RI of that variable bin. 


\subsection{Results and Discussion}

\subsubsection{Intensity Change Dependence on Motion and Shear}

It was noted in Chapter 3 and U14 that the phase of the surface wind asymmetry has a strong dependence on $V_{\text {storm }}$ and that major hurricanes experience more of an impact at the higher storm speeds. After removing the motion effects, it was also determined that the phase rotates cyclonically with increasing $V_{s h}$, except for major hurricanes. For the four intensity change groups, the same relationship is tested, and the results are provided in Figure 4.4. Linear regression fits are provided for $\alpha_{1, \max }$ as a function of $V_{\text {storm }}$ in the left panel and of $V_{\text {shr }}$ in the right panel. Motion has been removed from the analyses used in the right panel.
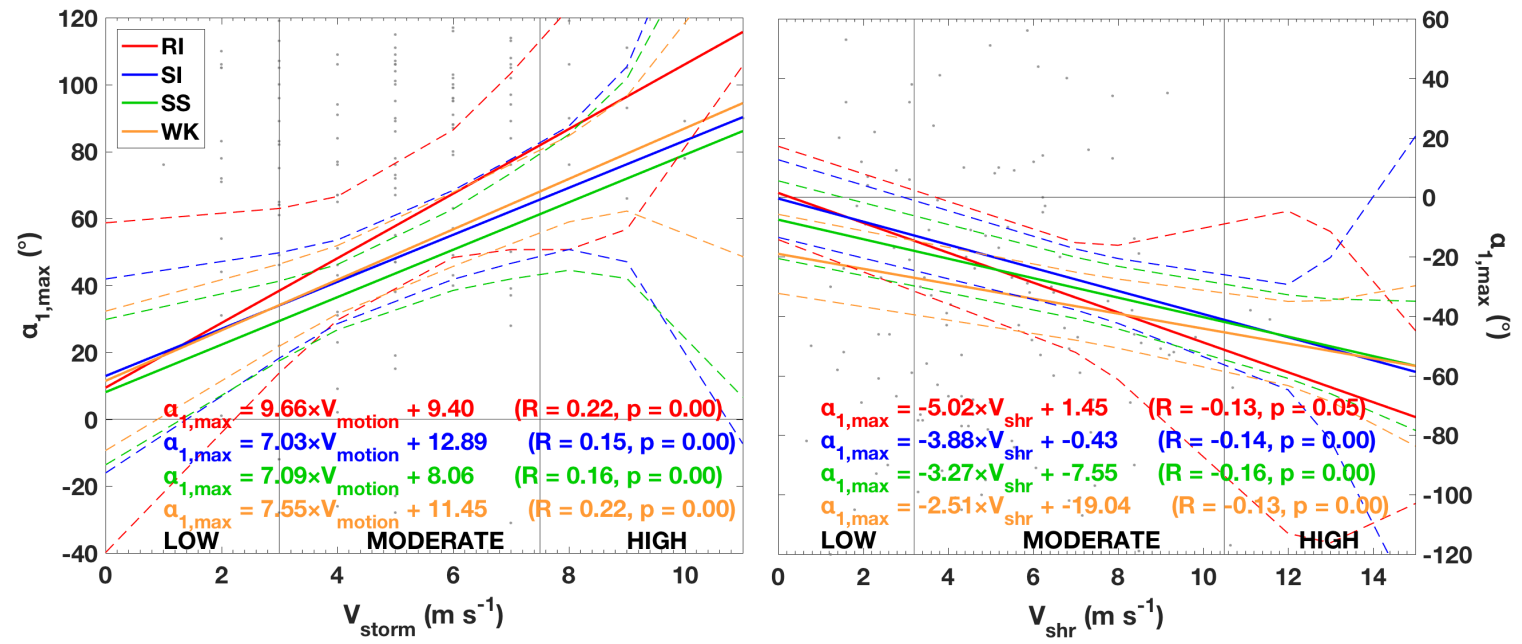

Figure 4.4. Linear regression fits of wavenumber-1 phase at the maximum amplitude $\left(\alpha_{1, \max }\right)$ are shown for the four intensity change groups. These are provided as a function of storm speed $\left(V_{\text {storm }}\right)$ without removing motion effects in the left panel and as a function of shear speed $\left(V_{s h r}\right)$ with motion effects removed in the right panel. The fit equations are also included and match the color for their respective line. Dashed lines represent the $95 \%$ confidence interval for each fit.

RI cases stand out in the motion-dependent fit as their phase rotates more per increase in $V_{\text {storm }}$ than the other three groups. There is no noticeable difference in fits for 
the non-RI groups as their slopes are nearly identical. Looking back at Figure 4.2, $V_{\text {shr }}$ tends to be less than about $6 \mathrm{~m} \mathrm{~s}^{-1}$ for the RI cases, where $\sim 70 \%$ of occurrences are at or below this shear speed. The SI group has a significant percentage of their cases below this value but also has more occurrence of shear higher than $8 \mathrm{~m} \mathrm{~s}^{-1}$. This increase in $V_{s h r}$ is likely the cause of the reduced dependency on $V_{\text {storm }}$. In the left panel of Figure 4.4 there is a clear cyclonic rotation of the maximum phase as in $\mathrm{KJ} 17$ and $\mathrm{U} 14$, but there is no significant difference between the intensity change groups. The RI cases show a slightly more shear-dependent relationship, but the $95 \%$ confidence intervals suggest these do not warrant any definitive difference compared to the other groups.

\subsubsection{Composite Analysis of Intensity Change Groups}

Based on the results in Figure 4.4, shear speed impacts the orientation of the low wavenumber wind speed analysis near the maximum amplitude in a similar fashion for all intensity change groups. KJ17 indicated that TC intensity shows the clearest trends in term of shear impacts, as tropical storms experienced the most influence and major hurricanes had negligible influence near the maximum amplitude. Because the mean $V_{\max }$ for three of the four intensity change groups are similar $\left(31,30,29\right.$, and $39 \mathrm{~m} \mathrm{~s}^{-1}$ for RI, SI, SS, and WK, respectively), this speaks to the response to shear seen in Figure 4.4. Notice that the WK group is more intense on average and that the response to increasing $V_{s h r}$ is lower than all other groups. The result here suggests that the regression fits are more of a reflection of the intensity of the $\mathrm{TC}$ rather than a direct relationship with the changing intensity. Two-dimensional, normalized composite low wavenumber fields are provided in Figure 4.5 for the four intensity change groups and indicate this similarity in the general structure. For the maximum, there is a slight cyclonic rotation from 
downshear-left (DSL) to left-of-shear (LS) from RI to WK cases. The WK TCs also are more asymmetric outside the RMW as the radial extent of stronger amplitudes decreases more quickly than for the other groups. Note that further reference to the shear-relative frame will include these abbreviations, where for example, upshear and right-of-shear are indicated by US and RS, respectively.
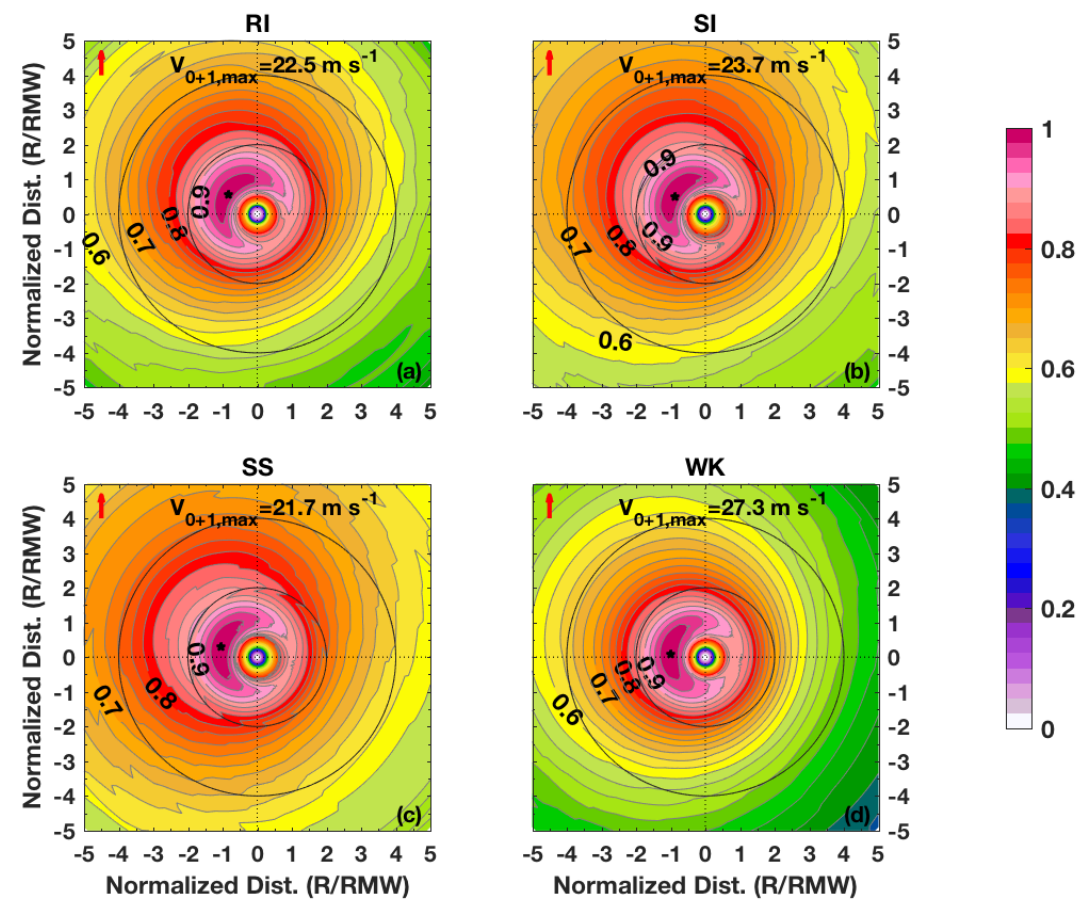

Figure 4.5. Two dimensional, low wavenumber analyses are provided for each intensity change group as a function of normalized radius (R/RMW). Wind speed amplitudes are normalized by the maximum amplitude on a scale from zero to one, where the maximum value is included at the top of the panel. Contours are plotted at intervals of 0.025 normalized units and the gray range rings indicate $2 \times$ and $4 \times$ RMW. The red arrow in each panel indicates the shear direction.

Quantification of the asymmetry is provided in Figure 4.6, where radial profiles of the low wavenumber asymmetry index (AIDX) are provided following the definitions in Alvey et al. (2015), Tao et al. (2017), and KJ17. It is clear that the overall AIDX is similar between the four groups [Figure 4.6(e)], although the RI cases are significantly 
more symmetric near the RMW compared to the SI cases. These profiles also indicate that INTS TCs (RI and SI) are generally more asymmetric than the NINTS (SS and WK) cases between 1-2.5×RMW. The INTS groups are twice as asymmetric as the NINTS on the right and left sides of the vortex at all radii as indicated in panels (a) and (b). Furthermore, the INTS groups are almost completely symmetric when comparing DSR and USL quadrants [Figure 4.6(c)]. This difference at first glance is a possible indicator for TC intensification. However, $8 \%$ of the INTS cases consist of major hurricanes (SI only) while the NINTS group contains over $20 \%$. This higher number of intense TCs provides reasoning for the more symmetric structure displayed in Figure 4.6. Because wind shear has a significant impact on the surface wind asymmetry (KJ17) and because the wind shear values are slightly but not significantly higher for non-intensifying cases, the overall shear-relative asymmetries are similar regardless of the type of intensity change. Despite the difference between the USL and DSR quadrants, there is no systematic difference in asymmetric structure, confirming the idea that the surface wind structure is more closely defined by the current intensity rather than the difference with a future intensity.

Another question in this same vein is related to the response of absolute angular momentum (AAM) transport between the intensity change groups. For reference, a description of AAM flux usage in this framework is provided in KJ17, where the main equations were derived from several previous studies (Pálmen and Riehl 1957; Holland 1983; Chan and Chan 2013). The analysis in KJ17 revealed that the maximum inward transport of angular momentum at the surface occurs $45-90^{\circ}$ upwind of the maximum low wavenumber amplitude in the presence of at least moderate wind shear. Generally, this 
was found in the DS or DSR quadrants as the intensity- and $\Delta \theta_{\mathrm{sm}}$-dependent composites exhibited an USL to DSL oriented asymmetric structure. Figure 4.7 provides the radial profiles of AAM flux in a shear-relative quadrant framework for the intensity change groups.
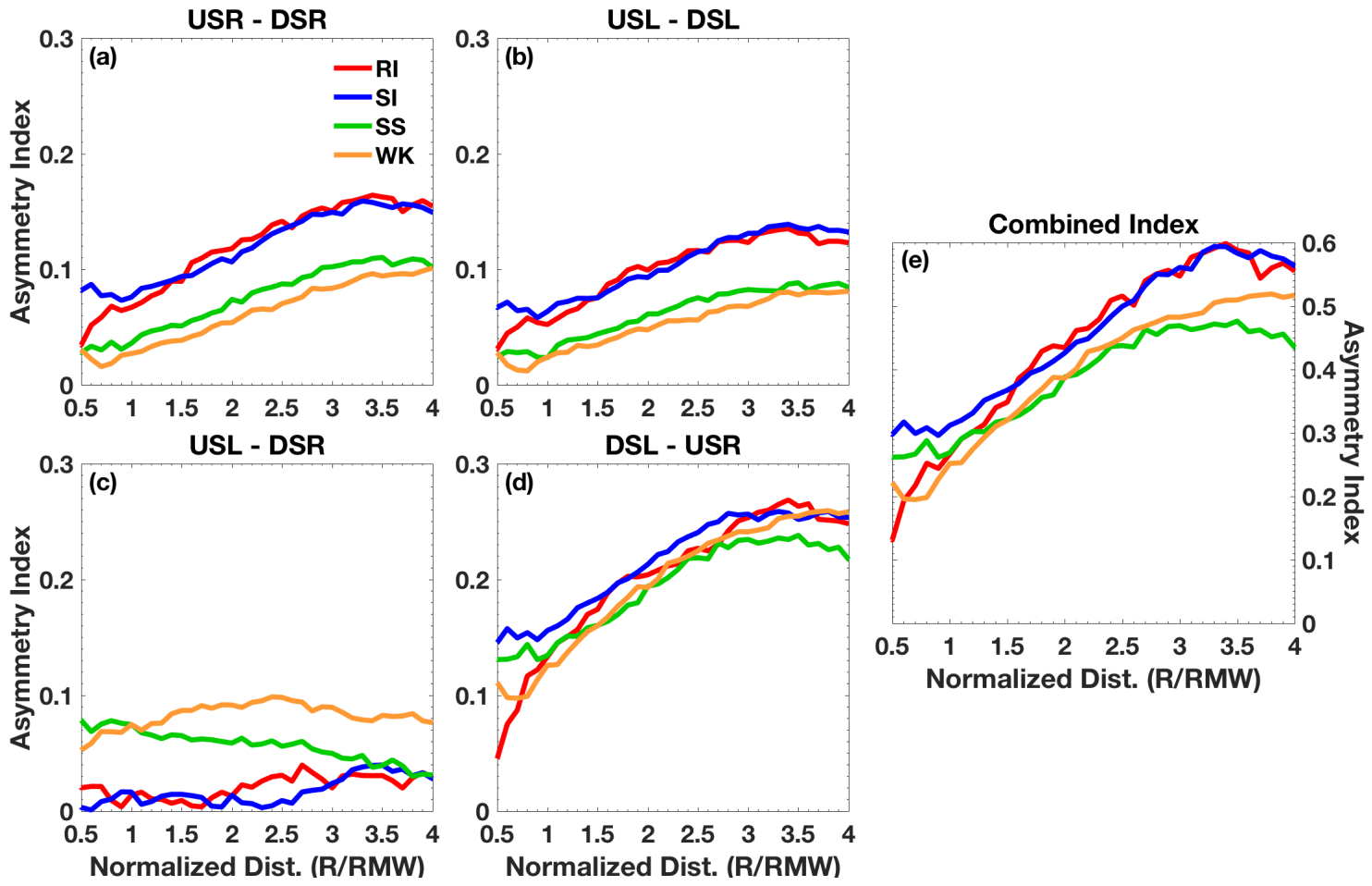

Figure 4.6. Radial profiles of wavenumber-1 asymmetry ratio $\left(V_{l} / V_{0+1}\right)$ are provided in panels (a-d) for four shear-relative quadrant absolute differences as a function of TC intensity change group. These profiles determine the overall asymmetry index, which is provided in (e). 'DS' and 'US' refer to down-shear and up-shear and the additional 'L' or ' $R$ ' indicates right or left, respectively.

The WK group has the largest AAM values but this is a result of the higher mean TC intensity due to the presence of more hurricane and major hurricane cases. The premise of the upwind location of the maximum AAM flux holds true as the INTS groups have their maximum flux DSR and maximum asymmetry DSL. The DSL and DSR quadrants for NINTS cases are similar in magnitude or favor the DSL quadrant (WK 
cases), which is consistent with a LS maximum surface wind asymmetry. The profiles match closely to their most common intensity contributor, where the RI and SI groups comprise $\sim 55-60 \%$ tropical storms and portray an AAM flux structure that lies between that of tropical storms and non-major hurricanes. This result is further confirmation of the suggestion that these composite asymmetric signatures are a result of $\mathrm{TC}$ intensity rather than intensity change.
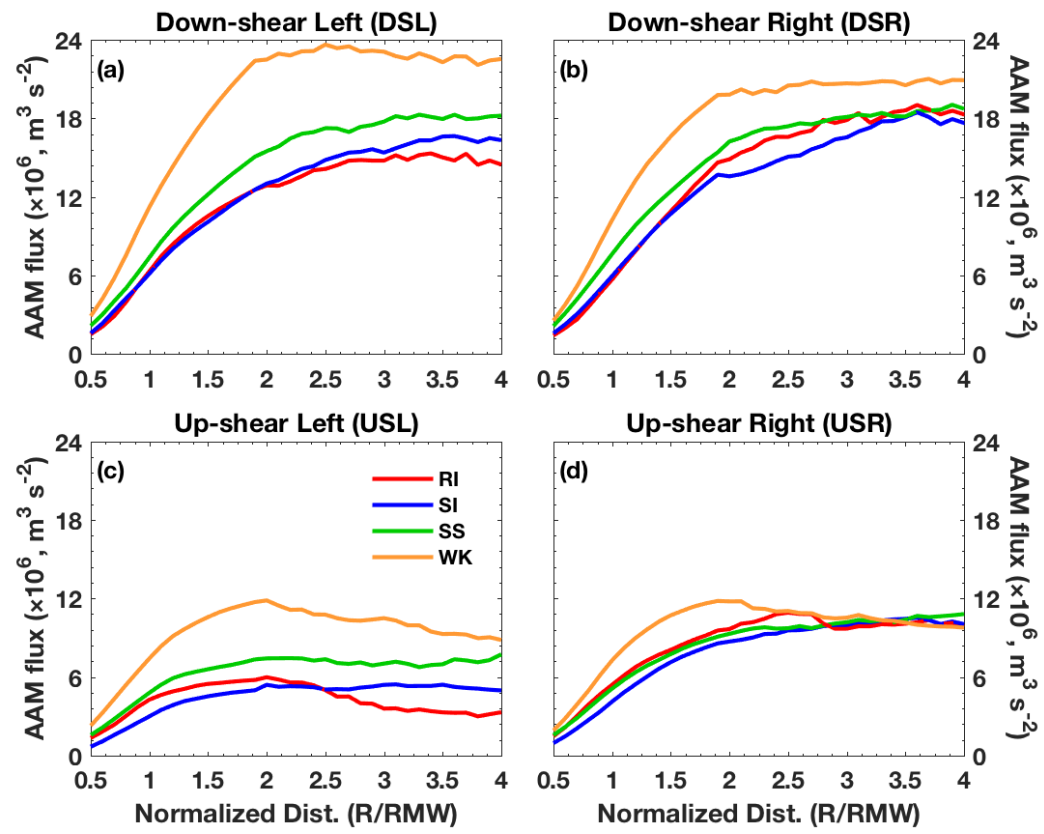

Figure 4.7. Absolute angular momentum flux (AAMF, $\left.1 \times 10^{6} \mathrm{~m}^{3} \mathrm{~s}^{-2}\right)$ ) radial profiles are shown with respect to shear-relative quadrants for the intensity change groups.

Another way to examine differences in structure in this framework is by looking at the RII and RIC composite results, which would indicate if there are differences between the initial stage of RI in relation to an established RI stage. Generally, the RIC cases have greater occurrence of low shear values (not shown), are stronger in intensity, and have shorter RMW. Maximum $\mathrm{V}_{0+1}$ is located DSL, with RII cases oriented slightly 
more LS. Basic statistics of these variables are provided in Table 4.3 and leads us to speculate the RII cases are more asymmetric due to the higher shear and weaker intensity.

Table 4.3. Provided are median and standard deviation values of various environmental and storm parameters within the RII and RIC groups. Note that $\alpha_{1, \max }$ is relative to the shear vector with storm motion removed.

\begin{tabular}{|c|c|c|c|c|c|c|}
\hline & RMW $(\mathrm{km})$ & $\mathrm{V}_{\text {shr }}\left(\mathrm{m} \mathrm{s}^{-1}\right)$ & $\mathrm{V}_{\text {storm }}\left(\mathrm{m} \mathrm{s}^{-1}\right)$ & $\Delta \theta_{\text {sm }}\left({ }^{\circ}\right)$ & $\mathrm{V}_{\max }\left(\mathrm{m} \mathrm{s}^{-1}\right)$ & $\alpha_{1, \max }\left(^{\circ}\right)$ \\
\hline RII & $63 \pm 24$ & $4.9 \pm 2.5$ & $5.0 \pm 2.2$ & $-24.0 \pm 87$ & $27.1 \pm 7.6$ & $-68 \pm 96$ \\
\hline RIC & $47 \pm 22$ & $4.5 \pm 2.3$ & $5.0 \pm 1.8$ & $-32.5 \pm 84$ & $33.0 \pm 8.2$ & $-59 \pm 87$ \\
\hline
\end{tabular}

To test this speculation, Figure 4.8 shows the shear-relative quadrant profiles of AIDX for RII and RIC conditions in a similar manner to Figure 4.6. With the exception of the DSL to USR comparison, the two classifications have similar radial profiles of AIDX. Notice that the DSL to USR asymmetry is systematically larger for RII cases (at 95\% significance), which provides initial confirmation that TCs become more symmetric in the surface winds during RI. The overall AIDX in Figure 4.8(e) also suggests this higher initial asymmetry through $\sim 1.5 \times \mathrm{RMW}$. Less confidence is present beyond this radius, but the overall result quantitatively suggests that TCs at the initial stage of RI are more asymmetric than later in the intensification process.

In terms of the AAM flux, Figure 4.9 provides the quadrant profiles as in Figure 4.7 but for RII and RIC stratifications. Comparing the two sets of profiles simply indicates that the difference in magnitude of the AAM flux is mostly due to the intensity of the vortex, which is obvious from the components used in the momentum calculation. Looking at the four profiles as a whole, the AAM flux is maximized at all radii DSR for the RII group, whereas the RIC group momentum flux maximizes DSL for radii inside 1.5-2 $\times$ RMW and DSR beyond $2 \times$ RMW. Both groups have minimum inward transport in 
the USL quadrant. The DSR (DSL) quadrant in the RII (RIC) group receives a boost from the presence of moderate shear, whereby the shear increases the influx of AAM and promotes the formation of the DSL (LS) low wavenumber maximum (not shown).
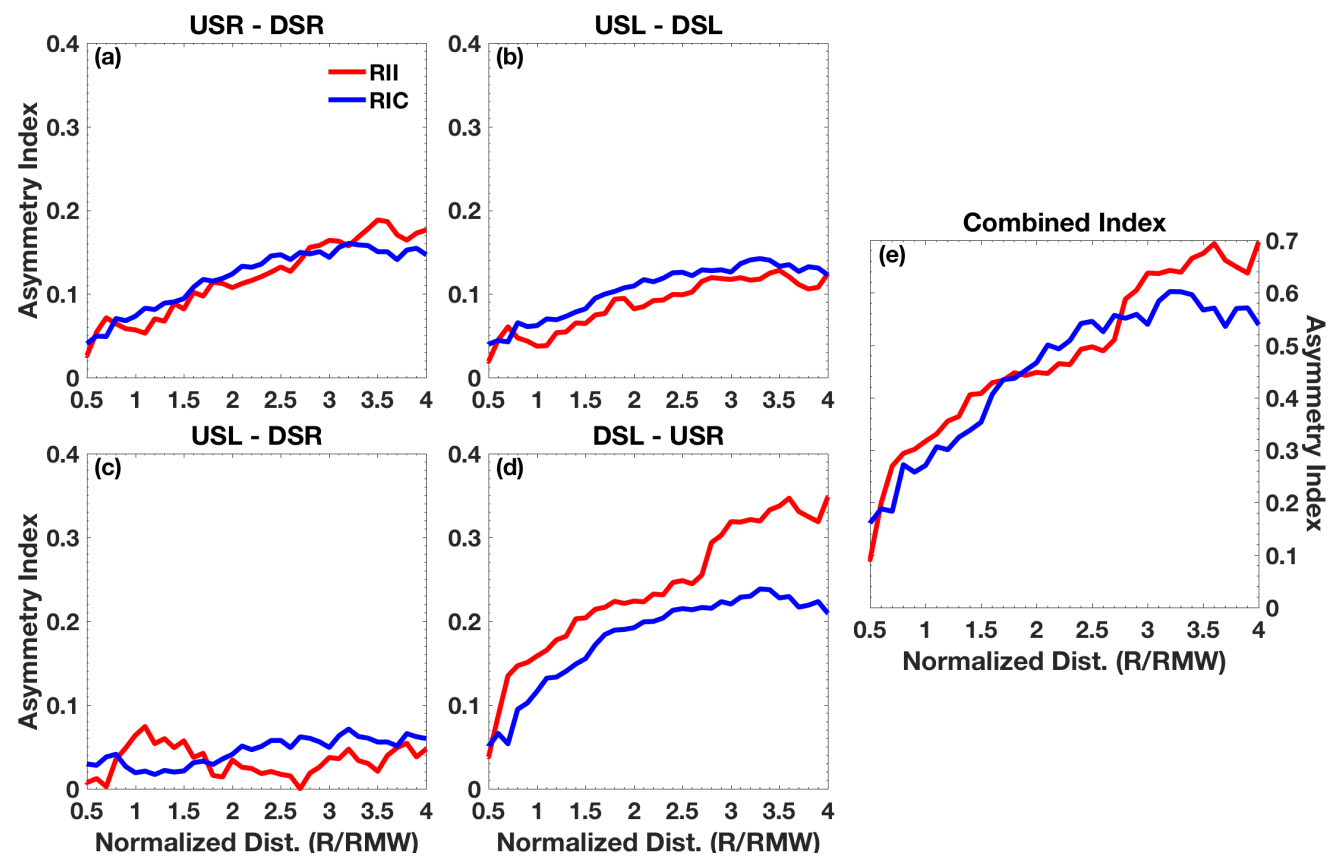

Figure 4.8. Similar to Figure 4.6 but for RII and RIC composites.

There seems to be minimal predictive power in terms of the differences between the RII and RIC groups in this framework. However, it is clear that wind shear has a controlling influence on the influx of AAM and the symmetrization of an intensifying vortex. Interestingly, the trends in the AAM flux between RII and RIC groups is similar to the trends associated with precipitation described in Tao and Jiang (2015) and Zagrodnik and Jiang (2014), where RII cases become more symmetric in all but the USR quadrant while RIC cases become more symmetric in all quadrants. 

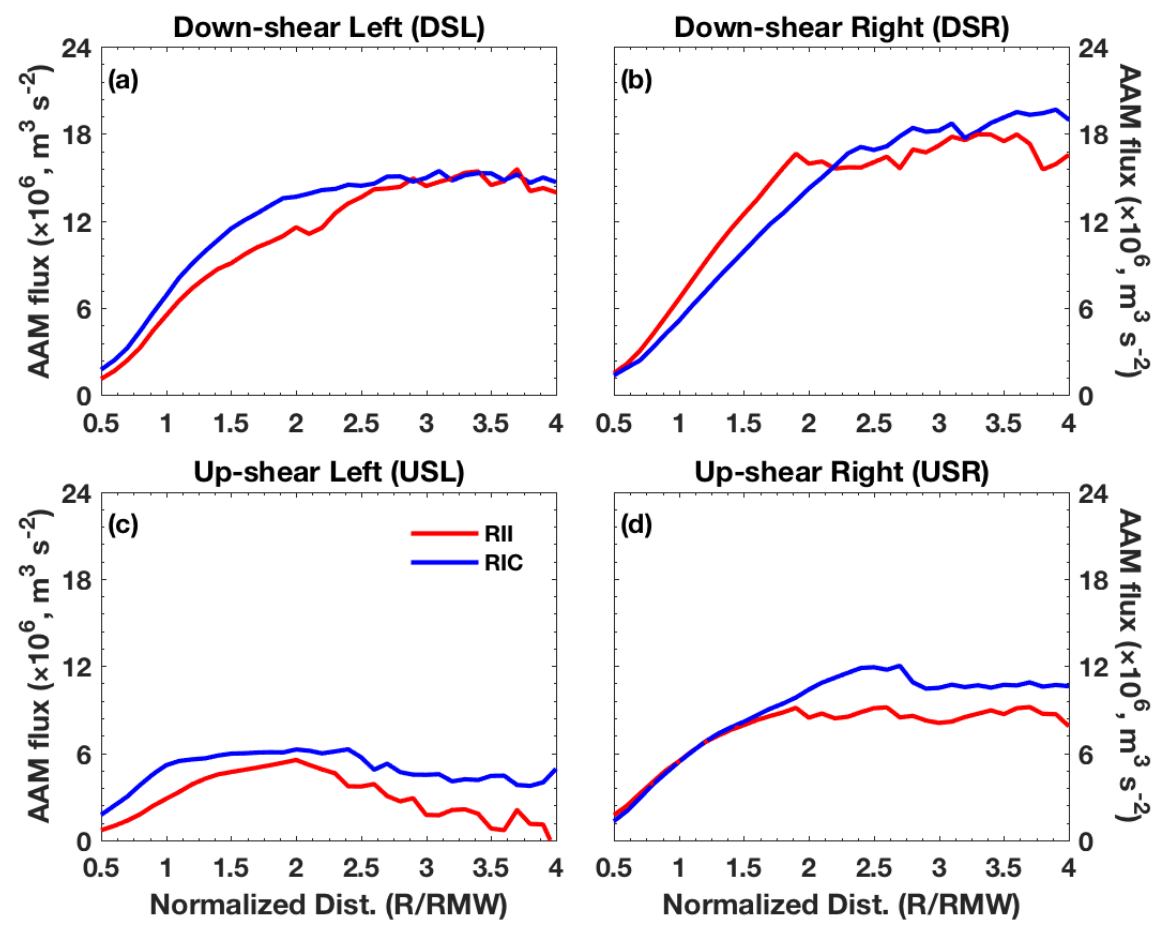

Figure 4.9. Similar to Figure 4.7 but for RII and RIC composites.

\subsubsection{An RI Event as Observed by QuikSCAT: Typhoon Parma}

The previous figures and discussion focused on the specific intensity change groups, but it is also beneficial to examine changes in asymmetry and its phase temporally through an RI event. As noted earlier, the RI event consists of a period prior to the initial RI time, the RI period itself, an ending period, and a post RI period. Before a general progression of the RI event is discussed, an examination of a single RI event is useful for understanding the perspective and ability of the scatterometer. Typhoon Parma, which was the $21^{\text {st }}$ named storm for the WPAC in 2003, was the only case in the full 12 year dataset that had observations at all periods within the definition of an RI event. Figure 4.10 shows the track of Parma with 00 and 12 UTC positions indicated along with the locations of the scatterometer center positions. Note that the time of the RI event, which began at 06 UTC on 22 October and ended at 12 UTC on 24 October (54 hour RI 
event duration), is marked with the bold line in Figure 4.10. It should be mentioned that Parma's track direction during the time of the RI period differs from the general track consensus of a northwest moving storm that gradually turns to the north by the end of the period.

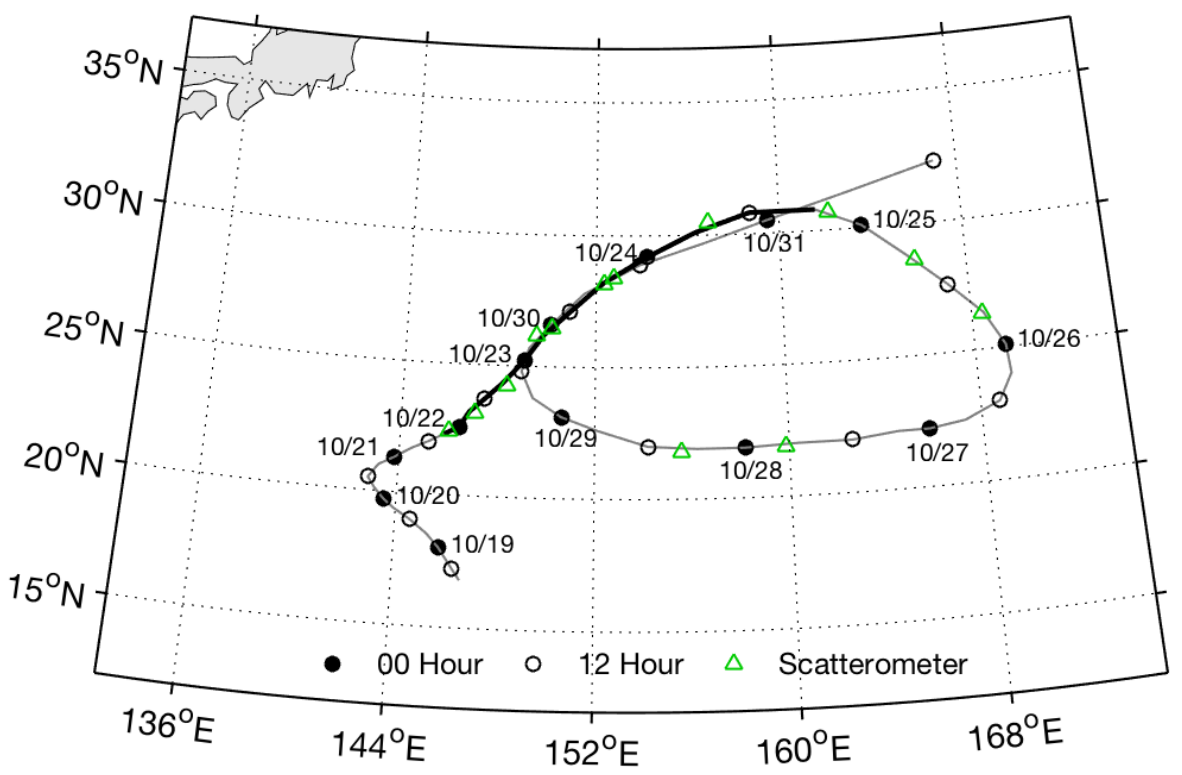

Figure 4.10. Presented is the track for Typhoon Parma (WP21, 2003) as taken from the best track data. Solid circles indicate times at 00 UTC while open circles indicate times at 12 UTC. Green triangles indicate center positions from the scatterometer overpasses, and the bold black line indicates the RI portion of the typhoon lifecycle.

Figure 4.11 provides several relevant and necessary storm and environmental parameters that put Typhoon Parma's RI event in perspective. As indicated by the $V_{\max }$ trace in Figure 4.11(a), Parma intensified from a moderate tropical storm to a Category 3 typhoon and further intensified (slowly) in the RI ending period to Category 4 intensity. Note that from the scatterometer analysis, the RMW contraction was delayed by about 18 hours from the RI initiation time. In theory, the rapid intensity change should occur in tandem with a reduced RMW, but Parma provides evidence that this is not always the case. The remaining three panels indicate that Parma was initially moving relatively 
slowly to the northeast and experiencing moderate shear from the southeast (oppositely pointing shear and motion). As the typhoon continued to the northeast, it picked up forward speed as the shear rotated to southerly, southwesterly, and eventually westerly by the end of the event. Interestingly the shear magnitude remained almost constant until the last 12 hours of the event. Parma presents the unique opportunity to completely examine the progression of the surface wind asymmetry as the constraints of the scatterometer generally only allow the observation of portions of but not full RI events.

To best view the change in structure through the progression of an RI event, Hovmöller-like plots of $V_{0}, V_{l}, \alpha_{l}$, and $V_{l} / V_{0+l}$ are presented in Figure 4.12. Panels (a) and (b) show normalized amplitudes based on the maximum value at each time, where the maximum value equals one. In Figure 4.12(a), the azimuthal mean $\left(V_{0}\right)$ amplitude is generally maximized near the RMW, but the period prior to RI initiation has an extended radial profile of the stronger amplitudes away from the RMW. For the asymmetric component before RI begins, the maximum amplitude is away from the RMW, however this area is likely not representative due to constraints of the analysis method. As the event moves forward, the maximum $V_{l}$ are found just inside the RMW, where their trace narrows as the storm intensifies.

By the RI ending period, the maximum asymmetric amplitude expands outside the RMW. The most important period for $V_{l}$ in this one case is the time between RI initiation and 24 hours prior to RI ending (between the first two dashed lines in each panel of Figure 4.12). Early in the period, the maximum area of high $V_{l}$ is expansive near the RMW and at least $40-50 \%$ of the maximum is observed up to $2 \times$ RMW. During the second time window while the storm is intensifying, the amplitude appears to only 
contribute within the RMW. Panel (d) indicates that the asymmetric contribution is between $30-40 \%$ of the total amplitude in this later period and $0-10 \%$ outside the RMW. This trend is indicative of a system that is becoming more symmetric as it intensifies.
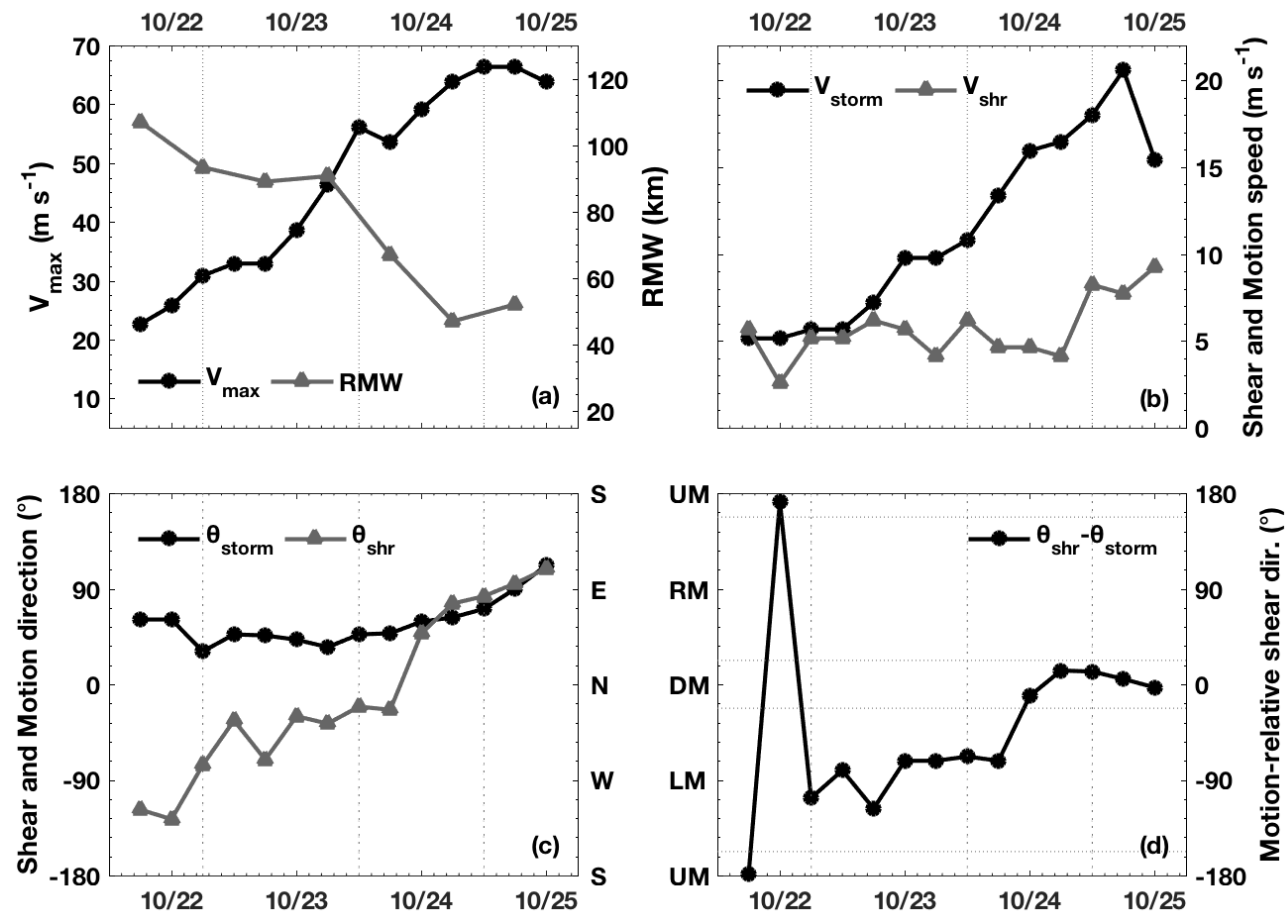

Figure 4.11. In (a), the time series of $V_{\max }$ (black line) from the best track data and RMW as determined from the scatterometer data (gray line) are plotted for Typhoon Parma. In (b), the storm motion (black) and wind shear (gray) speed are plotted, and in (c), their respective directions are shown, where $\theta_{\text {shr }}$ of $0^{\circ}$ (or $\mathrm{N}$ ) indicates shear directed from south to north. In (d), the difference in the shear and motion angle is provided. Note that the time period is only for 12 hours prior to and after the RI event as denoted in Figure 4.10. Vertical dotted lines in all panels mark the initiation, start of the ending period, and the end of the RI event, respectively. The horizontal dotted lines in (d) represent the angle difference bins as described in KJ17 (Chapter 3).

The phase trace through the event is somewhat noisy but reveals that near the RMW, the wavenumber-1 field is oriented US but fluctuates between RS and US by $\mathrm{RI}_{\mathrm{E}^{-}}$ 24 (and somewhat USL at extended radii). During the RI ending period, the phase rotates clockwise to the DSL quadrant. The results are consistent with the composites presented in KJ17 that found oppositely pointing $V_{\text {shr }}$ and $V_{\text {storm }}$ contribute to an USL or US 
oriented wind field. As the wind shear and forward motion increase in speed and align in direction, the results match the expected composite value, where the asymmetric field is oriented DSL. For Typhoon Parma, $V_{l}$ and $\alpha_{1}$ follow the trends expected from the composite analyses, where the TC is highly asymmetric prior to RI onset and is significantly impacted by the orientation of shear and motion. During the RI period, the asymmetry outside the RMW is reduced and near the RMW is consolidated as the orientation rotates clockwise in conjunction with a clockwise rotation of the shear and alignment with the motion vector.
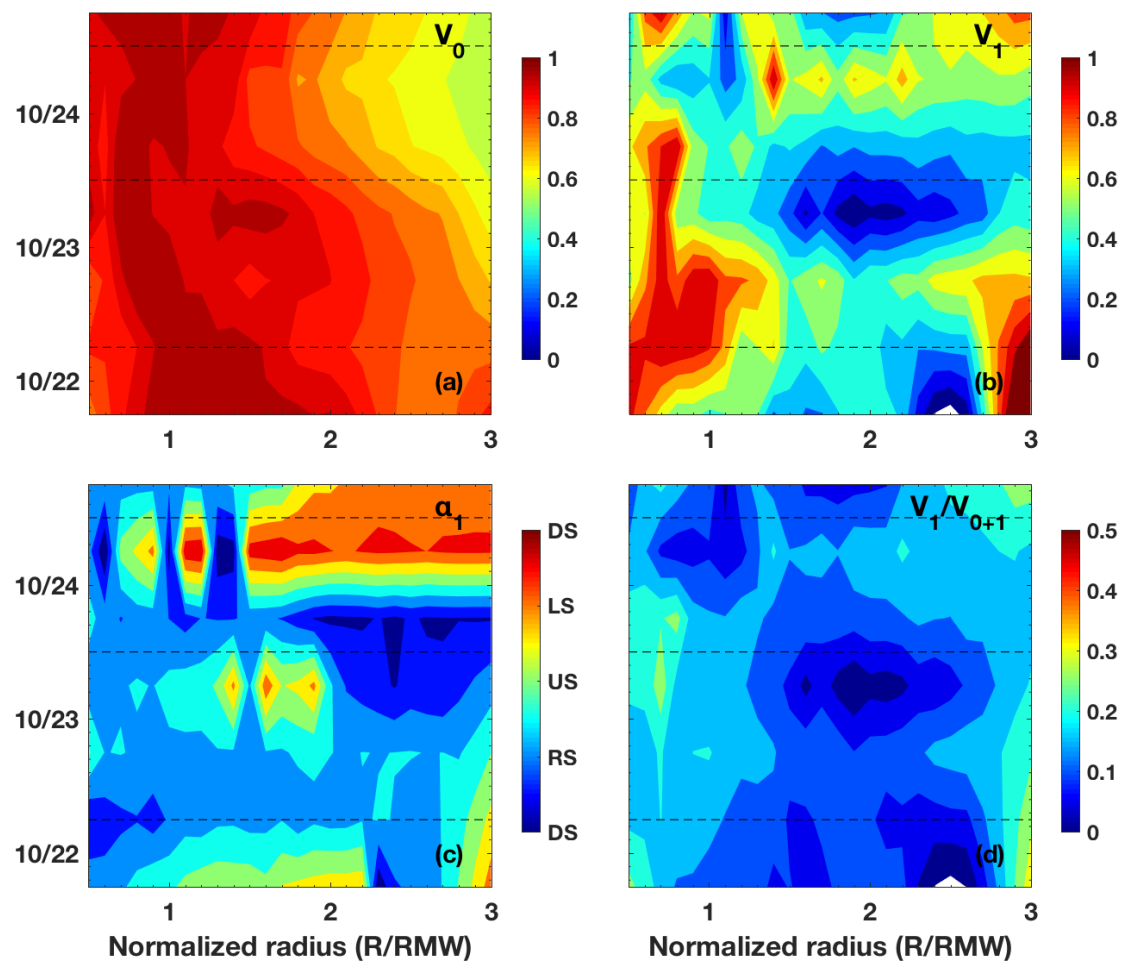

Figure 4.12. A time-radius plot for (a) normalized wavenumber-0, (b) normalized wavenumber-1 amplitude, (c) wavenumber-1 phase, and (d) wavenumber-1 percentage of the total amplitude is shown for the Typhoon Parma RI event. The three dashed lines mark the same RI-relevant periods as noted in Figure 4.11. 


\subsubsection{Composite RI Event}

While the Typhoon Parma case is an interesting depiction of an RI event, it is not necessarily representative of the general progression expected. In this section, composite analyses of the various RI onset relative periods are used to describe the general progression of the surface wind asymmetry during rapid intensity change. Environmental and storm influences for the composite groups are shown in Figure 4.13 similarly to Figure 4.11, where the RMW contracts as the storm intensifies and $V_{s h r}$ weakens leading up to and during RI before increasing again after RI ends. Shear also tends to be slightly left of motion prior to RI onset but generally aligns with motion later in the RI progression. The relation to motion is an important factor in the determination of the phase of the wind asymmetry as shown with Typhoon Parma.
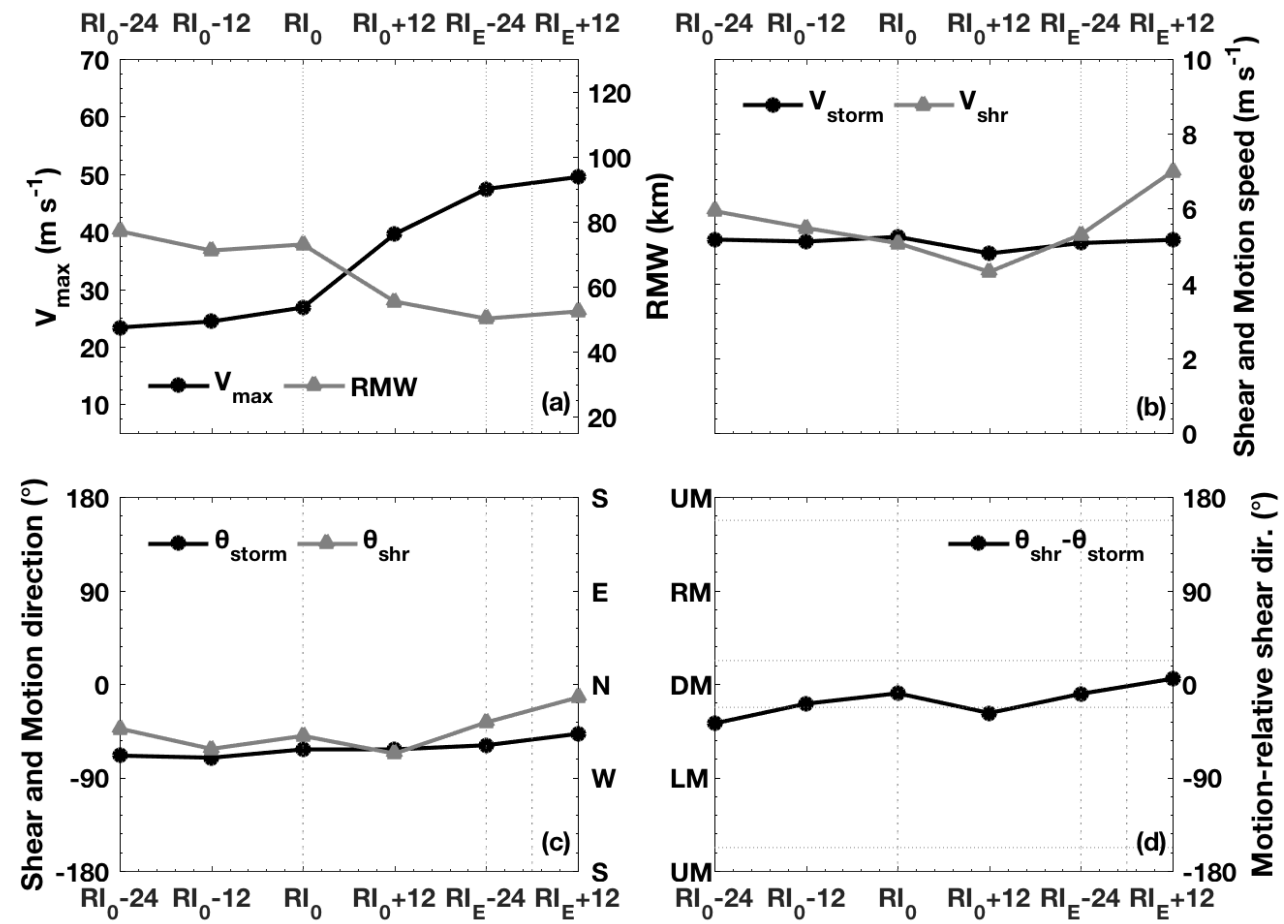

Figure 4.13. Similar to Figure 4.11 but for the composite RI event timeline. Values plotted represent medians for the respective variables. 
It is worthwhile to first quantify the shear-relative asymmetry for each RI event period. Although not shown, the two-dimensional fields for each of these event-based periods is generally oriented DSL, with some variation. Figure 4.14 provides the AIDX radial profiles as in Figure 4.6. In the overall AIDX shown in Figure 4.14(e), notice that the period prior to RI initiation has the largest asymmetry at most radii. Comparing the overall AIDX in the radial range between $1-2.5 \times \mathrm{RMW}$, the tendency is for the TC surface winds to become more symmetric through an event. Also notice that within the first 12 hours of an RI event, the asymmetry is reduced by $\sim 50 \%$ of the pre-RI value at the RMW. This difference is statistically significant at $95 \%$. The asymmetry ratio continues to decrease through the continuing phase of the event and is most symmetric during the RI ending period. As shear begins to increase and storms remain steady state or weaken, the asymmetry generally increases again after an RI event ends. The period prior to RI (red line) is on average $\sim 1.5$ times more asymmetric and as high as 4 times larger near the RMW when comparing to the RI ending period (orange line). These profiles confirm the hypothesis that the asymmetry decreases during TC rapid intensification. It is also clear that regions away from the RMW are susceptible to influences from wind shear throughout the event (although less affected at later RI periods).

Figure 4.15 provides the time-radius images of $V_{0}, V_{l}, \alpha_{1}$, and $V_{l} / V_{0+l}$ as in Figure 4.12 but for the composite RI event. Clearly, the wavenumber- 0 amplitude is maximized near the RMW at all periods, and the radial extent of higher values decreases as the TC intensifies. The asymmetry amplitude as with Typhoon Parma is largest beyond $2 \times \mathrm{RMW}$ before RI begins and is weaker near the maximum $V_{0}$. As RI initiates, a double maximum 
develops where the $V_{l}$ influence is strong at extended radii but is also becoming stronger near the RMW. By the tail end of the RI continuing period and through the RI ending period, the asymmetric amplitude consolidates and maximizes near the RMW, which signifies the amplitude reduction of the wavenumber-1 asymmetry at extended radii. Panel (d), however, indicates that the $V_{I}$ percentage of the total amplitude is still higher beyond $2 \times \mathrm{RMW}$, which suggests that the rate of reduction of $V_{0}$ at these radii is quicker than for $V_{l}$. The takeaway here is that early in the RI event, the surface wind field is highly asymmetric at all radii but as the TC intensifies, the asymmetric structure is reduced at extended radii and enhanced near the RMW.
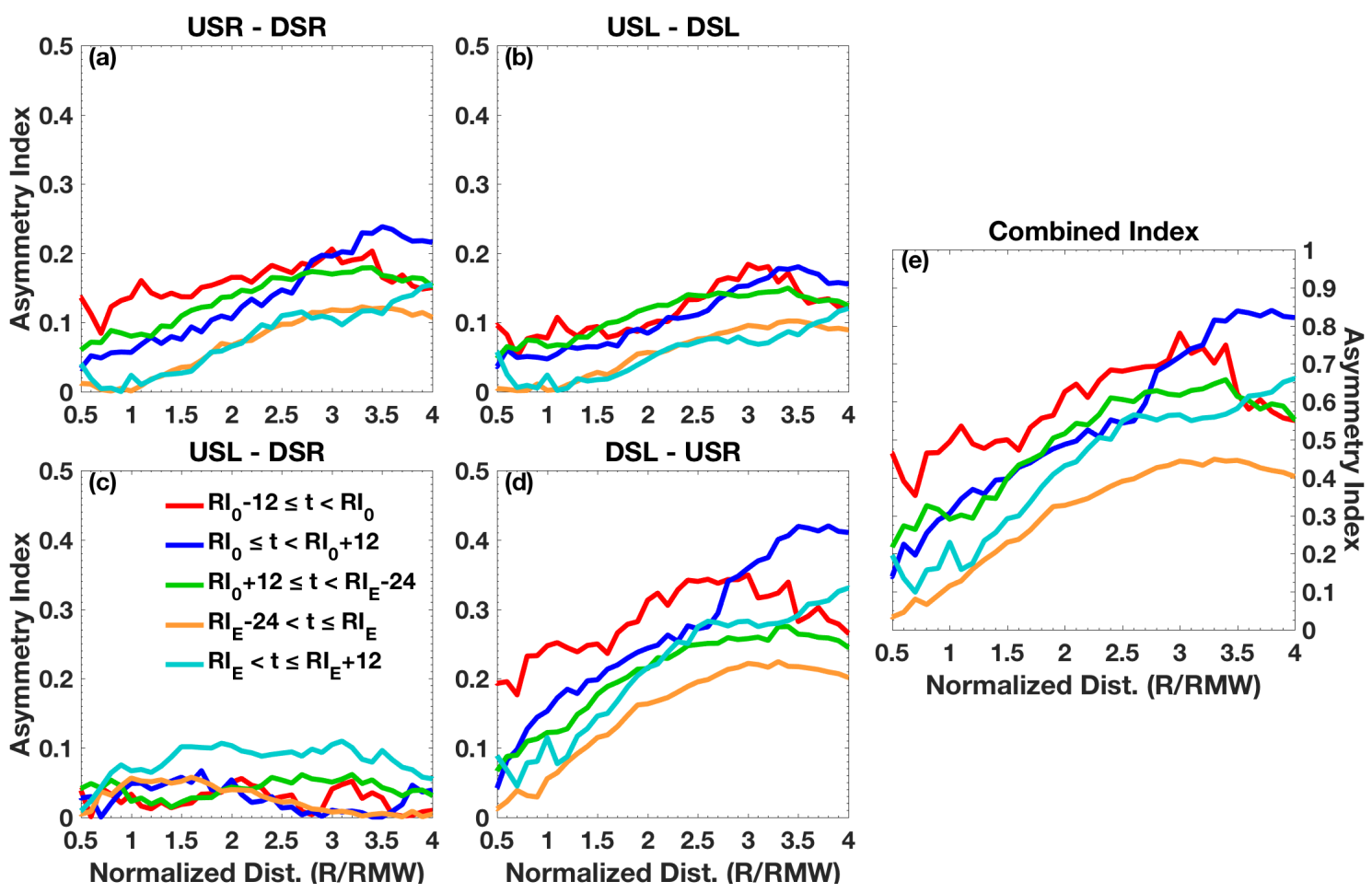

Figure 4.14. Similar to Figure 4.6 but for the designated RI event periods.

Similarly to the low wavenumber amplitude, the wavenumber-1 phase is provided in Figure 4.15(c) to showcase the change in shear-relative orientation of the surface wind asymmetry. During the pre-RI and RI initiation times, the phase fluctuates between DSL 
and LS at all radii, but the phase near the RMW is slightly more downwind than at outer radii. During the RI continuing period and through the RI ending period, the phase rotates from DSL to LS to slightly USL near the RMW with the outer radii rotated more in the DS direction, comparatively. The significant rotation of the phase from initiation to ending is a result of an increase in $V_{s h}$, consistent with the results in Figure 4.4 and KJ17. Interestingly, this large change in phase near the RMW is quicker than the change at outer radii, suggesting that the phase of the asymmetric field becomes more disjointed during an RI event and coincides with the results in KJ17 where intense TCs have a significantly different response to shear near the RMW than at outer radii. In weaker TCs, the response to shear is similar at all radii.
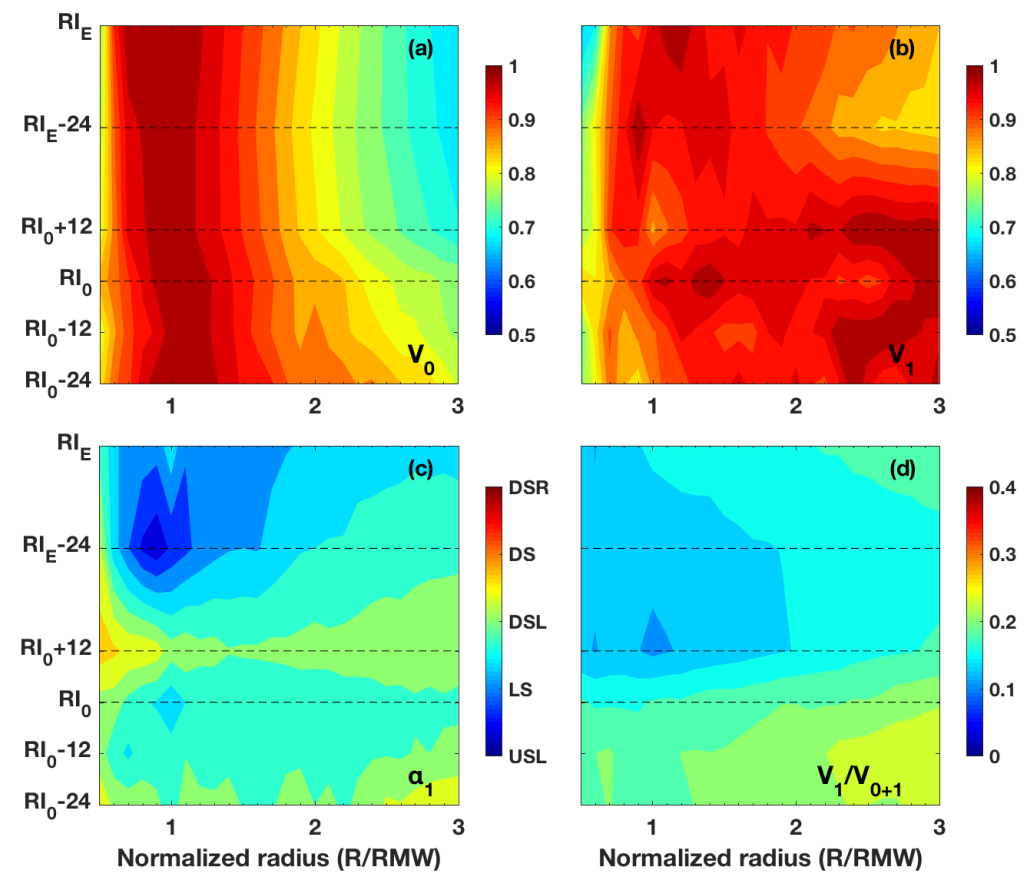

Figure 4.15. Similar to Figure 4.12 but for the composite RI event progression.

It is clear that the shear influence on AAM flux has implications for orientation of the surface wind field in various circumstances. One common characteristic yet to be discussed is the sharp gradient of AAM flux values from inside the RMW through 2 - 
$2.5 \times$ RMW. In conjunction with the time evolution of the RI event, the progression of the gradient of AAM flux is depicted in Figure 4.16. There is an apparent relationship between the maximum flux gradient and RI, where prior to RI, the largest gradient is beyond the RMW. As the TC begins to intensify, the gradient moves inward toward the RMW and continues inward through the end of RI. AAM is often viewed with a vertical component as an explanation of the TC secondary circulation (i.e. Chan and Chan 2013). While Figure 4.16 is a product of AAM flux at the TC surface, it describes a simple principle that the maximum gradient of surface AAM flux must occur at or within the RMW to ensure a robust secondary circulation and promote intensification. While not shown, the quadrant profiles for the AAM flux follow similar trends to the intensity groups in $\mathrm{KJ} 17$, where the maximum flux is upwind of the maximum low wavenumber asymmetry. It is clear that a significant difference in the inward flux of AAM is present at the start of RI when compared to the times leading up to RI. After the RI initiates, AAM flux is more a result of the TC intensity itself rather than the changing of intensity.

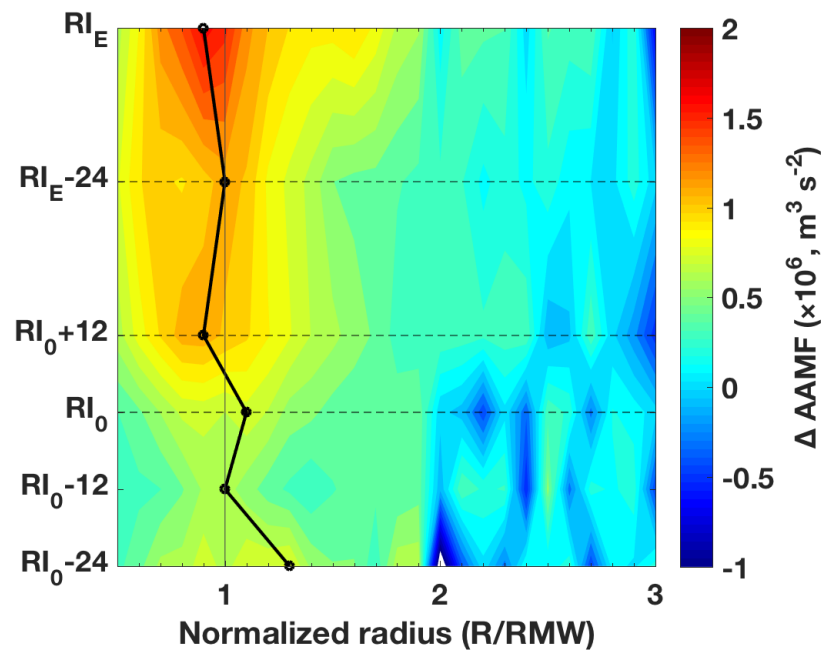

Figure 4.16. A time-radius plot of the gradient of AAM flux is provided for the composite RI event times. The solid black line is the location of the maximum AAM flux gradient for a particular time. The dashed lines represent the times over which RI occurs. 


\subsubsection{Discussion}

The processes involved with initiating RI are manifested in the AAM flux trace as seen in Figure 4.16, but what is the mechanism that consolidates the flux towards the RMW and begins to reduce the asymmetric structure at outer radii? In turn, what role does the low wavenumber surface wind asymmetry play in this process? One possible connection is related to the theories involving formation of intense convection near or within the RMW as a way to provide a latent heating source near the TC center. Related studies to this theory point to formation of $\mathrm{CBs}$ or convective towers near or within the RMW in association with RI (Montgomery et al. 2009; Rogers et al. 2015, 2016), but because the convective regions are transient in nature, Jiang (2012) and Tao and Jiang (2015) suggest that the entire precipitation/convective field is a better indicator of the initiation of RI. They found using TRMM precipitation radar data that significant increases of shallow and moderate convection/precipitation were present in the US quadrants only during the RI initial and continuing periods compared to either non- or slowly-intensifying TCs. The highest percentages were found generally well away from the TC center (and most likely outside the RMW). Even when using a degraded resolution similar to TRMM TMI, they notice this same trend in the precipitation coverage associated with RI.

Using the TMI rain data, it is possible to quantitatively compare the location of the rain rate to the maximum low wavenumber asymmetry. According to UK09, the maximum wind speed near the surface is theoretically expected to be located $\sim 90^{\circ}$ downwind of the maximum convective region (or upward motion) in a motion relative framework. Because of the rotation of the vortex, inflow in the boundary layer, and the 
presence of shear, the maximum rain rate in theory would manifest slightly downwind and radially inward of the of this upward motion. Figure 4.17 depicts how these parameters are related from the perspective of an RI event.
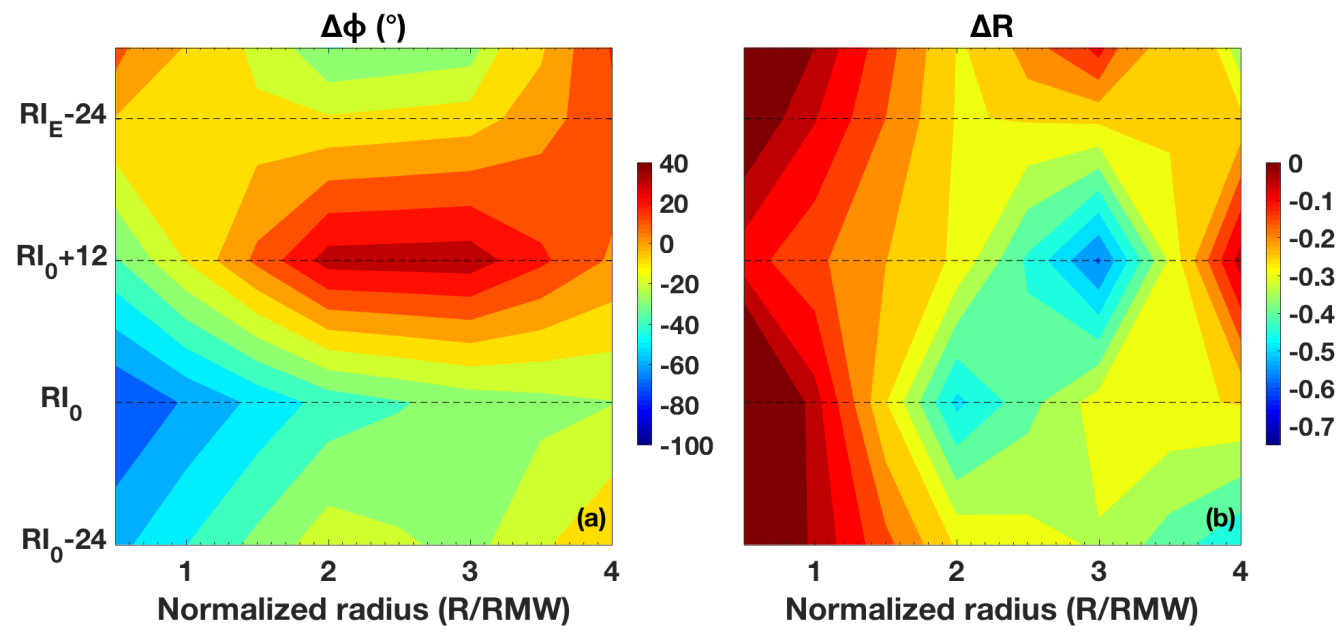

Figure 4.17. Time-radius images are shown for (a) the azimuthal difference between the maximum low wavenumber amplitude and maximum TMI rain rate, and (b) the normalized radius difference between the maximum wind and rain parameters in (a). Negative values in (a) indicate the low wavenumber phase is downwind of the maximum rain rate azimuth while negative values in (b) indicate a radial location of the maximum rain rate outward of the surface wind radius. The slight difference in time labels compared to Figure 4.16 is due to the constraints in the TMI dataset.

In panel (a), the azimuthal difference of the wavenumber-1 phase and location of the maximum rain rate $\left(\Delta \phi=\alpha_{1, \max }-\phi_{t m i}\right)$ indicates that before RI begins, $\alpha_{1}$ is located between $40-60^{\circ}$ downwind of the maximum rain rate. During this time period, the normalized radial difference in panel (b), which is the difference in distance between the maximum wind and rain parameters $\left(\Delta R=R_{\alpha l}-R_{t m i}\right)$, indicates that the rain rate is slightly farther away from the TC center. In general, increasing the normalized radius is associated with an increased distance between the rain and wind parameters. In other words, near the RMW, maximum rain features are generally well-located radially with their associated wind maxima but extend further away from them at larger radii. 
It is confirmed from the rain and wind result that while the presence of deep convection near the RMW as suggested by Rogers et al. $(2015,2016)$ is involved in the RI initiation, significantly increasing the coverage of precipitation at all strengths may be needed for RI initiation. The suggestion here is that for the time prior to and during RI onset, a significant increase in moisture in all quadrants (relative to non-RI cases, Zawislak et al. 2016) associated with large rain areas and in conjunction with an inward increase of AAM flux near the RMW provides a mechanism by which the TC secondary circulation can develop and make a way for intensification to occur. This would then indicate that the low wavenumber asymmetry amplitude and orientation at this time is not only related to the influence of moderate wind shear but that it is also an effect of other upwind, storm-related processes rather than a cause. After the initial 12 hours of the RI period, the most distinguishable difference in the event progression is with $\Delta \phi$, where the maximum rain rate and maximum low wavenumber amplitude become mostly aligned near the RMW by the start of the RI ending period. It is clear that precipitation/convection, thermodynamic, and kinematic processes are all intertwined at the initiation of RI and that variations in the low wavenumber asymmetry are dependent on variations of these influences.

\subsection{Summary and Conclusions}

Examination of the surface wind asymmetry from an observational perspective to date has been limited to specific basins and has only focused on hurricanes. U14 examined low wavenumber asymmetry from an aircraft perspective using SFMR data, but due to database size, they could not quantify specific relationships between intensity or intensity change. UK09 and UB11 used scatterometer wind data similarly to this 
present study but were also limited in sample size and scope. Because of this lack of complete analysis of the full intensity spectrum as well as a detailed response to wind shear, KJ17 developed an analysis method specific for scatterometer winds and quantified the asymmetry related to various intensity groups as well as the influence of shear on this asymmetric structure.

As a follow-on work to $\mathrm{KJ} 17$, this present study takes a different approach by examining and quantifying the asymmetric structure related to TC intensity change, specifically looking at composites of different intensity change groups ranging from rapidly intensifying to weakening TCs. Additionally, examination of the initial and continuing periods of RI (Zagrodnik and Jiang 2014) as well as presentation of a composite RI event portray unique and interesting characteristics of the low wavenumber structure and how it changes in association with RI initiation. The difficulty of definitively determining why changes in structure are found is to due to the complexity of the precipitation, thermodynamic, and kinematic processes associated with a rotating and intensifying vortex. A relationship between overlapping rain data from TMI and low wavenumber amplitude from scatterometer during an RI event suggests that the asymmetric structure is a result of thermodynamic and precipitation processes. From the results and discussion presented, the main conclusions are:

- Intensity change composites for RI, SI, SS, and WK groups and their related environmental (i.e. $V_{s h r}$ ) and storm statistics (i.e. $V_{\max }$, RMW) reveal that the asymmetric structure is most closely related to the initial intensity rather than a change in intensity. Their associated shear-relative, quadrant profiles do not show any statistically significant difference as the response to increasing shear is similar. 
- Examination of RII and RIC cases reveals that changes in low wavenumber structure is again related more to initial intensity, but RIC cases are more symmetric overall and in terms of AAM flux in all shear-relative quadrants.

- Depiction of a composite RI event from periods prior to RI onset to RI ending indicate that $V_{l}$ is very broad initially but contracts near and inside the RMW as a TC undergoes intensification. Orientation of the phase near the RMW is generally downshear-left at the early RI event stages but quickly rotates left of shear to upshear-left as shear begins to increase in strength near the end of an RI event. Additionally, the maximum gradient of AAM flux must align with or occur inward of the RMW for RI to occur.

- Comparison with maximum rain rate reveals that the maximum wind asymmetry is downwind of the coincident rain rate and is radially well-located with the precipitation, which suggests that surface wind asymmetry is likely more of an effect from the precipitation and thermodynamic process instead of a cause.

The analysis of surface wind asymmetries from scatterometer winds is useful for many reasons regarding better understanding of the associated physical processes. One remaining question is can these analyses be used in some predictive or verification capacity? Vukicevic et al. (2014) compared the low wavenumber maximum from a TC model against those determined from SFMR data and proposed their use as an alternative method of forecast verification. A next step here is to reproduce a similar comparison but with the scatterometer data. If a robust signal is determined, this type of analysis could be useful for verifying model intensities as well as the best track data. 


\section{PREDICTABILITY AND VERIFICATION OF SURFACE WIND STRUCTURE IN TROPICAL CYCLONES}

\subsection{Abstract}

Prediction of tropical cyclone intensity is a complicated procedure and certain inherent errors and biases exist in both objective and subjective predictions. Verification of the model intensity metric with the subjective and sometimes uncertain operational forecast techniques is often not ideal, and an existing study using aircraft data provided evidence that a low wavenumber metric was reproducible and had predictive skill. Because of the rarity of aircraft data in most global tropical cyclone basins, this study examines the usefulness similar analyses from corrected satellite-based scatterometer analyses, and discusses its predictive skill for intensity against the standard operational 'best track' metric. These analyses are stratified by storm intensity, intensity change, and tropical cyclone basin. Results indicate skill for tropical storms and non-major hurricanes and reliability in the North Atlantic and Eastern North Pacific basins with caveats in the Western North Pacific and Southern Hemisphere. Additionally, the scatterometer metric performs better compared to best track metric through 48-60 hours of a given forecast but deteriorate in predictive skill beyond 72 hours due to model uncertainty and generally increasing intensity. A preliminary analysis of the surface wind structure relative to rain in the context of intensity change reveals predictive skill for non-intensifying tropical cyclones.

\subsection{Introduction}

Because of the destructive characteristics of TCs, diligent monitoring of their formation, progression, and intensification is necessary not only for the safety of 
impacted populations but also for the preservation of property and coastal integrity. TCs have the ability to cause damage due to strong winds and flooding due to storm surge and inland inundation from torrential rains. With consideration of these factors, accurate observing systems are required to diagnose the destructive potential of a TC. Observations, however, only provide a status update of the current state of a TC, and it is necessary to use modeling systems (both statistical and dynamical) to help diagnose the future location and strength of a TC.

On the observational side, aircraft reconnaissance is the most trusted medium for the current intensity, position, and motion parameters. Since 2005, implementation of an annual NOAA hurricane field program focuses on intensity processes associated with TCs. Known as the Intensity Forecast Experiment (IFEX, Rogers et al. 2006, Rogers et al. 2013), a diverse and often unique suite of data are collected with the intent of improving observing capabilities as well as understanding the processes associated with TC intensification. Globally, aircraft reconnaissance is mostly non-existent, although, several field programs were conducted over the Western North Pacific basin during the last decade, including Tropical Cyclone Structure 2008 (TCS-08, Elsberry and Harr 2008) and Impact of Typhoons on the Ocean in the Pacific (ITOP, D'Asaro et al. 2014). However, there is no regular field campaign aimed at collecting reconnaissance data in TCs in the Western Pacific or Indian Oceans (Northern or Southern Hemisphere).

With the emergence of weather satellites in the 1970s, initial position and motion characteristics could be determined without aircraft data, but intensity estimates were still highly questionable. Using infrared imagery, a technique that incorporates information about varying cloud structures was developed to provide an estimate of TC intensity 
(Dvorak 1975; 1984). The Dvorak technique is currently a significant contributor to intensity estimates and is available in both user-defined and automated formats (Velden et al. 2006; Olander and Velden 2007). Remarkably, the technique is reliable in most conditions, but Knaff et al. (2010) notes that there are higher uncertainties at the edges of the intensity spectrum (i.e., weak tropical storms and Category 4+ hurricanes). Cangialosi et al. (2015) also indicates that the Dvorak estimate for storms undergoing intensity change performs well within the constraints of the technique when aircraft reconnaissance is available. Due to the fact that in a forecast setting previous forecasts are used to assess the current Dvorak intensity estimate, inherent biases related to aircraft reconnaissance and subjective interpretation are present as well.

Advancements and additions of data sources since the development of the Dvorak technique as well as use of other proxies of surface wind speed (Kossin et al .2007; Knaff et al. 2011) contribute to a forecasters ability to make a decision about the current intensity. By taking these data into consideration, a 'best track' dataset is produced for each storm in a given basin (using the specified operating procedures for said basin's forecast center). Figure 1 of Landsea and Franklin (2013) provides an example of the diversity of observations that are utilized during the production of a best track data point for TC intensity. Note that in their Table 2, the uncertainty of using satellite only for intensity increases for stronger hurricanes on average, but they indicate that the absolute uncertainty is largest for tropical storms $(\sim 25 \%)$. It is clear from their results that at least $10 \mathrm{kt}$ of uncertainty from satellite only estimate is expected regardless of the TC strength. Also note that when aircraft data are present, the uncertainty is only slightly lower, ranging between $8-10 \mathrm{kt}$. 
It is obvious that providing accurate information about a TC's current status has its limitations. However, another operational requirement for forecast centers is their provision of a future position and strength, generally out to five days from the initial time. To accomplish this task, forecasters rely heavily on dynamical, statistical, and consensus models (DeMaria et al. 2009) to gain some kind of understanding about the future storm- and environmental-specific characteristics that will impact the development and progression of a TC. For intensity purposes, a push for better high-resolution, mesoscale models led to improvements of the Geophysical Fluid Dynamics Laboratory (GFDL) Hurricane model (Kurihara and Tuleya 1974; Bender et al. 2007) and the Hurricane Weather Research and Forecast Model (HWRF, Tallapragada et al. 2014), which is based on the parent model WRF-ARW (Skamarock et al. 2008). WRF-ARW incorporates processes defined in a Nonhydrostatic Mesoscale Model (NMM) system (Janjic 2003). Advances on the implementation and representativeness of HWRF (Davis et al. 2010; X. Zhang et al. 2011; Gopalakrishnan et al. 2011; 2012) as part of the Hurricane Forecast Improvement Project (HFIP, Gall et al. 2013) have provided a slight improvement to intensity forecasts, but there remains a wide range of variability in the accuracy of these forecasts. Increasing the model resolution to account for smaller scale processes is possible, but the computing power needed to perform a similar five or seven day forecast increases significantly, making them operationally unfeasible.

As part of the suite of products produced by these regional models, 10-m wind speeds (surface winds) are provided and can be used to perform verification of model TC intensity. Standard verification is performed against the hurricane best track intensity as indicated above (Vukicevic et al. 2014, referred to as V14), but it is arguably 
unreasonable to expect model intensities to align with a metric that in itself is uncertain, especially for different types of TCs and conditions where only satellite data are utilized. Processes that may be captured in some of the data may also be unresolvable by regional hurricane models due to their horizontal resolution $(\mathrm{O} 3 \mathrm{~km})$. V14 proposed an alternative method of forecast intensity verification using a Fourier decomposed model wind field that evaluates the maximum amplitude against similar products from observational resources. Ultimately, V14 determined that the predictability of a TC is well-confined to a wavenumber- 0 and -1 estimate with the knowledge of a residual term within the expected uncertainty of the best-track estimates (Torn and Snyder 2010; Landsea and Franklin 2013).

As noted in a Chapter 2, the SFMR dataset from Uhlhorn et al. (2014, referred to as U14) and V14 is representative of a general trend but is too small to diagnose differences associated with TC intensity or intensity change. Using the low wavenumber analyses from the scatterometer data, it is feasible that these estimates can be provided. Therefore, this portion of the study seeks to evaluate the estimate of TC intensity first against the best track and Dvorak estimates and then against those produced by HWRF model output. The observational comparisons are produced for the overall dataset, by TC basin, by TC intensity, and TC intensity change classifications. Further evaluation against model is performed with the scatterometer data similarly to V14, where comparisons are performed at forecast lead times out to five days. The purpose here is to prove (or disprove) the usefulness of the scatterometer data for regional model intensity verification. The sections of the study are as follows: A description of the data and methods are provided in section 5.3 followed by a comparison of the scatterometer data 
against best track and Dvorak intensity estimates in section 5.4. A fourth section examines the usefulness of the scatterometer for model verification and a fifth section provides a discussion of some preliminary analysis of rain relative to the asymmetric surface wind structure. The final section provides some conclusions based on the results.

\subsection{Data and Methods}

\subsubsection{Description of Best Track and Dvorak Data and Their Use}

Because the best track data has been discussed in previous chapters, it is unnecessary to provide any more details here. Effectively, the best track intensity is reported at six hour intervals and is designed to represent a maximum 10-m, 1-minute sustained wind speed (Jarvinen et al. 1984). Each scatterometer case was matched with a best track intensity value, which will be used to evaluate the low wavenumber amplitude. The Dvorak data are obtained from the NHC or JTWC f-deck or "fix" archives. These archived files contain clerical information such as the satellite instrument used to make a TC fix (i.e., infrared, microwave, etc) or the agency that produced the estimate (i.e., Satellite Analysis Branch or SAB). Pertinent Dvorak intensity estimates are provided in the form of the analysis T-number and associated current intensity (CI) value (Dvorak 1984). Each CI number covers a wind speed range between $2.5 \mathrm{~m} \mathrm{~s}^{-1}$ and $7.5 \mathrm{~m} \mathrm{~s}^{-1}$, where ranges increase with increasing storm intensity. Knaff et al. (2010) notes that there is more uncertainty at higher intensities due to the range increase as well as limitations of the technique. For comparison purposes, these intensity estimates are matched with the closest best track time.

To evaluate the performance of the low wavenumber technique from the scatterometer against the best track, a linear regression fit of the data is performed. 
Additional regression fits of the SFMR low wavenumber data from U14 as well as intensity estimates from Dvorak data are produced for reference and for assessing the value of the scatterometer analyses. Probability density functions (PDFs) of the differences between the best track and respective data source are also provided and effectively represent the $\varepsilon$ (or residual term) from Eq. 3.1. The PDF of the Dvorak estimate is included as well but is only representative of the frequency of intensity estimates relative to the best track. Stratifying according to storm intensity and intensity change for these fits and PDFs allows examination of the conditions where the scatterometer performs well and conditions where it is not as useful. These stratifications are only calculated for the Fourier decomposed analyses because Knaff et al. (2010) discusses the expected biases associated with the Dvorak estimates in terms of intensity and intensity change, where a high and low bias are found for weakening and intensifying TCs, respectively. The linear regression fit and residual PDF from the model low wavenumber analyses (discussed in the following subsection) are provided for reference.

\subsubsection{HWRF Data and Methods}

For the model data, six hourly output from 5-day retrospective runs of the 2015 operational version of the HWRF model are used (Tallapragada et al. 2015). These output files are obtained for the 2011 season for both the North Atlantic and Eastern North Pacific basins from the mass storage archive on NOAA's Jet supercomputer. HWRF is triply-nested with horizontal domain resolutions of 18,6 , and $2 \mathrm{~km}$, which is a significant upgrade from previous model versions. The grids, from largest to smallest, spatially cover areas of $80^{\circ} \times 80^{\circ}, 12^{\circ} \times 12^{\circ}$, and $7.1^{\circ} \times 7.1^{\circ}$, respectively. Additionally, the inner two nests are designed to initially center on the TC and follow the vortex throughout the 
model run. Figure 5.1 provides a graphical view of the size of the three domains. For the inset panel featuring the inner two domains, the range rings increase from 50 to $250 \mathrm{~km}$ with increments of $100 \mathrm{~km}$ to show the size of the vortex inner core relative to the grid size. The outer ring is $500 \mathrm{~km}$ from the storm center to show the extent of the region where the vortex (environmental) flow becomes less (more) influential. As in any numerical simulation, certain physical schemes and parameterizations are necessary for forming a TC. Table 5.1 lists the various physical schemes and parameterizations used in HWRF, some of which are new to the 2015 version.
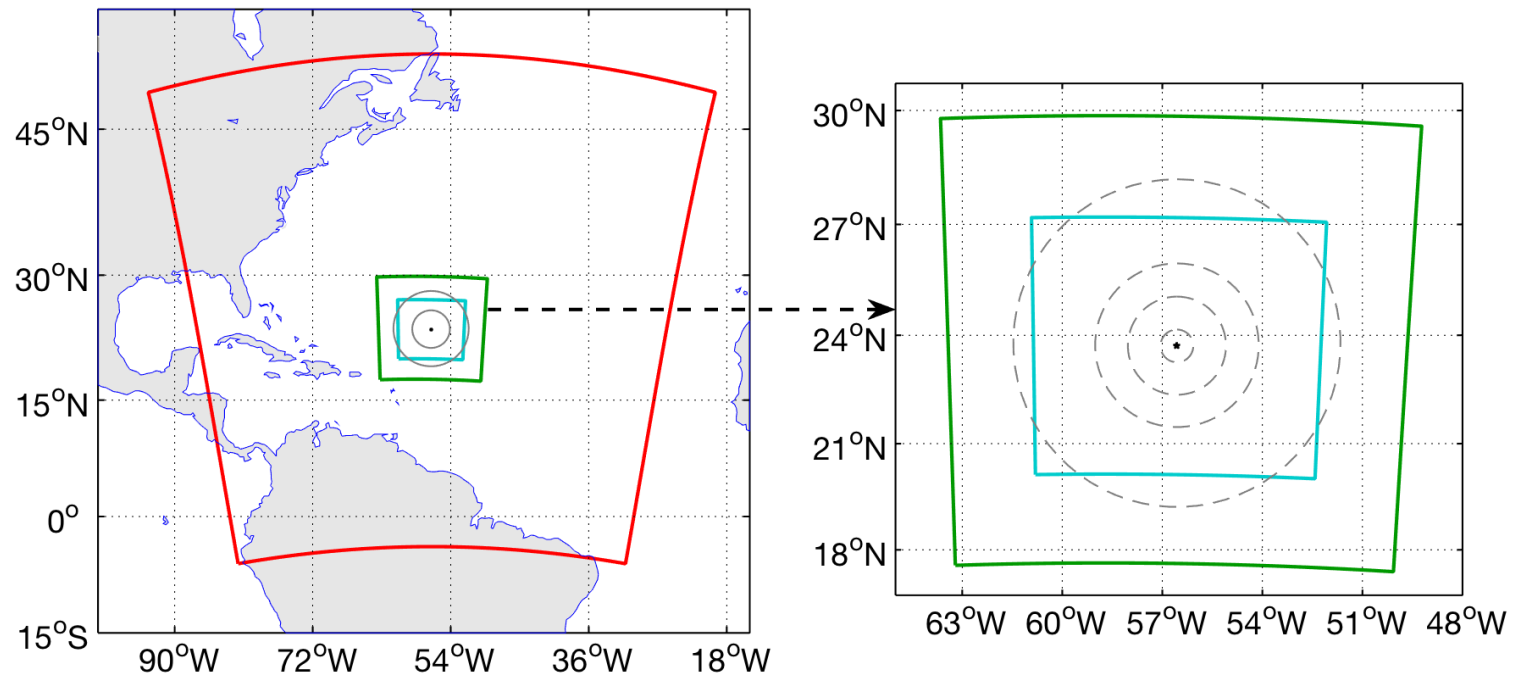

Figure 5.1. In the left panel, the three nested grids for the 2015 version of HWRF are provided for the outer (red), middle (green), and inner (blue) domains. The inset panel provides a magnified view of the two inner domains, where dashed rings indicate radial distances of 50,150,250, and $500 \mathrm{~km}$ from the TC center.

Initial variables produced by HWRF are placed on a staggered latitude/longitude E-grid, which can be difficult to work with in a TC framework. Therefore, output data were post-processed using the Hurricane Research Division diagnostic postprocessor (Diapost), which interpolates the meteorological variables to cylindrical coordinates while also keeping track of automated tropical cyclone forecasting (ATCF) type products 
(X. Zhang et al. 2011). These are similar to what are found in the best track dataset provided by HURDAT2 (Landsea and Franklin 2013). For the purposes of this study, 10$\mathrm{m}$ wind speed fields within the innermost domain are used.

Table 5.1. Provided is a list of the different physical schemes and parameterizations used in the 2015 operational version of HWRF. Entries marked with an asterisk are new to this version. A list of related references is provided for each scheme or parameterization.

\begin{tabular}{|c|c|c|}
\hline Microphysics & Scheme/Parameterization & Reference \\
\hline Cumulus & $\begin{array}{c}\text { Simplified Arakawa } \\
\text { Schubert (SAS) }\end{array}$ & Aligo et al. (2014) \\
\hline Planetary Boundary Layer & Modified GFS & $\begin{array}{c}\text { Hong and Pan (1996), Bu } \\
\text { (2015) }\end{array}$ \\
\hline Surface Layer & Modified GFDL & $\begin{array}{c}\text { Powell et al. (2003), Kwon } \\
\text { et al. (2010) }\end{array}$ \\
\hline Radiation & $\begin{array}{c}\text { Rapid Radiative Transfer } \\
\text { for General Circulation } \\
\text { Models (RRTMG)* }\end{array}$ & Iacono et al. (2008) \\
\hline Land Surface & Noah Land Surface Model & Chen and Dudhia (2001) \\
\hline
\end{tabular}

Additionally, the vertical depth of the model atmosphere stretches over as many as 76 vertical levels in the 2015 version of HWRF (Tallapragada et al. 2015), but the troposphere contains 61 levels defined on a pressure/eta surface. Horizontal winds at the equivalent 850 and $200 \mathrm{hPa}$ levels within the outer domain are used to estimate an environmental wind shear value, similar to the shear provided by the SHIPS statistical model. Shear values are calculated within several radial rings beyond $300 \mathrm{~km}$ to ensure influences from the vortex circulation are negligible. This wind shear estimate can then be used to evaluate any results relative to a shear influence.

Unlike most observation systems, numerical models can be considered a complete entity in terms of kinematic, thermodynamic, or precipitation variables. In this regard, 
one final data quantity that is used in this study is the $10-\mathrm{m}$ rain rate. Providing an estimate of the rain in terms of location and strength is useful for relating to results from the surface wind speed analysis either in the earth-relative or shear-relative reference frame. Additionally, a form of verification of the results in Chapter 4 can be provided through use of this model and Diapost post-processed data.

The methodology performed for this portion of the study follows closely to the methods described in V14. Standard Fourier decomposition of the 10-m wind speed is performed, and a resulting model wavenumber-0 amplitude $\left(V_{m, 0}\right)$ and wavenumber-1 amplitude and phase $\left(V_{m, 1}\right.$ and $\left.\alpha_{m, 1}\right)$ is given. A residual term ( $\varepsilon$, see Eq. 3.1$)$ is used to represent the remaining wavenumbers of the Fourier decomposition and is a relatively reliable estimate of uncertainty. Note that a subscript $m$ here indicates it is calculated from model output. At this stage, the maximum combined amplitude $\left(V_{m, 0}+V_{m, 1}\right)$ is calculated and is used to define the RMW as well as represent the intensity metric to compare against a representative best track intensity (ATCF formatted, 10-m maximum forecasted wind speed) and observational low wavenumber amplitude.

Comparison of the low wavenumber maximum amplitude can be evaluated for each output time against the ATCF best track estimate. However, it is also beneficial to temporally match the scatterometer maximum amplitude with the model, which can be performed on a case-by-case basis or as a function of forecast lead time. The equations follow a simple arithmetic difference between the observational and model quantity. Evaluation of the standard metrics is also performed as in V14, where differences between the forecast $V_{\max }$ and matched observational best track $V_{\max }$ are calculated as indicated by Eq 5.1a and b (modified versions of V14 equations 7a and b): 


$$
\begin{aligned}
& \left\langle V_{m, \text { max }}-V_{s, B T}\right\rangle=\left\langle V_{m, 0}-V_{s, 0}\right\rangle+\left\langle V_{m, 1}-V_{s, 1}\right\rangle+\left\langle\varepsilon_{m}-\varepsilon_{s}\right\rangle, \\
& \left\langle\left|V_{m, \max }-V_{s, B T}\right|\right\rangle=\left\langle\left|V_{m, 0}-V_{s, 0}\right|\right\rangle+\left\langle\left|V_{m, 1}-V_{s, 1}\right|\right\rangle+\left\langle\left|\varepsilon_{m}-\varepsilon_{s}\right|\right\rangle .
\end{aligned}
$$

Braces indicate average values, and subscripts $m$ and $s$ refer to model and scatterometer entities, respectively. Any biases in the differences can be removed by subtracting the mean error from the mean absolute error (i.e. 5.1b - 5.1a). Note that $\mathrm{V}_{\mathrm{s}, \mathrm{BT}}$ is the best track intensity or associated $V_{\max }$. Table 5.2 provides the list of TCs for each basin and the number of HWRF runs that are available as well as the number of runs that have available scatterometer data for comparison. There are 115 individual scatterometer cases used for matching with the 2011 HWRF data.

As noted previously, it is possible to use the coincident rain and wind data to assess the relationship between their radial and azimuthal location. For the purpose of this dissertation, the location of the maximum rain rate $\left(\phi_{r r, \max }\right)$ is determined from the cylindrical grid and is compared to the phase of the maximum low wavenumber amplitude $\left(\Delta \phi=\alpha_{1, \max }-\phi_{r r, \max }\right)$. While rain rate is not entirely representative of convective processes, it provides a basic understanding of the general relationship between precipitation and winds at the surface. Confirmation of the theoretical discourse (Ueno and Kunii 2009) can be tested as well as an evaluation of similar observation-based analyses presented in Chapter 4. 
Table 5.2. Listed are the model cases and number of runs available from the HWRF dataset for North Atlantic and Eastern Pacific TCs. Also included is the number of cases with at least one overlapping scatterometer time.

\begin{tabular}{|c|c|c|c|}
\hline \multicolumn{4}{|c|}{ North Atlantic cases } \\
\hline Name & Storm Number & Number of cases & Overlapping cases \\
\hline Arlene & AL01 & 11 & 4 \\
\hline Bret & AL02 & 21 & 16 \\
\hline Cindy & AL03 & 12 & 12 \\
\hline Don & AL04 & 13 & 3 \\
\hline Emily & AL05 & 30 & 27 \\
\hline Franklin & AL06 & 7 & 7 \\
\hline Gert & AL07 & 14 & 14 \\
\hline Harvey & AL08 & 17 & 4 \\
\hline Irene & AL09 & 37 & 30 \\
\hline Ten & AL10 & 9 & 0 \\
\hline Jose & AL11 & 12 & 11 \\
\hline Katia & AL12 & 52 & 48 \\
\hline Lee & AL13 & 19 & 0 \\
\hline Maria & AL14 & 42 & 40 \\
\hline Nate & AL15 & 19 & 10 \\
\hline Ophelia & AL16 & 53 & 48 \\
\hline Philippe & AL17 & 62 & 59 \\
\hline Totals & ---- & 427 & 333 \\
\hline \multicolumn{4}{|c|}{ Eastern Pacific cases } \\
\hline Adrian & EP01 & 21 & 18 \\
\hline Beatriz & EP02 & 12 & 7 \\
\hline Calvin & EP03 & 11 & 11 \\
\hline Dora & EP04 & 28 & 24 \\
\hline Eugene & EP05 & 28 & 28 \\
\hline Fernanda & EP06 & 23 & 23 \\
\hline Greg & EP07 & 21 & 10 \\
\hline Eight & EP08 & 4 & 0 \\
\hline Hilary & EP09 & 40 & 35 \\
\hline Jova & EP10 & 30 & 21 \\
\hline Irwin & EP11 & 45 & 44 \\
\hline Twelve & EP12 & 7 & 0 \\
\hline Totals & --- & 270 & 221 \\
\hline
\end{tabular}


5.4 Scatterometer TC Intensity Metric Compared to Similar Best Track, Dvorak, and HWRF Estimates

\subsubsection{Overall Trends}

Following the methods above, an assessment of the usefulness of the scatterometer low wavenumber maximum intensity (i.e. $V_{0+1, \max }$ ) is provided for all scatterometer cases used in Chapters 2-4. Figure 5.2(a) shows the matched intensity pairs and linear regression fits to the official best track data for the scatterometer, SFMR, and HWRF low wavenumber analyses. The fit of the Dvorak intensity estimate is also provided for reference. As indicated in the figure, the scatterometer data has the lowest coefficient of determination, where $80 \%$ of the variance is explained by the fit. To contrast this value, the SFMR and model pairs explain $91 \%$ and $96 \%$ of the variance, respectively. It is noticeable that as intensity increases beyond $\sim 50 \mathrm{~m} \mathrm{~s}^{-1}$, the scatterometer difference with the best track intensity increases to the point where it is likely not reliable. The strong wind result is not surprising considering the horizontal resolution limitations and constraints of the correction technique applied to the scatterometer data.

Looking at the PDFs in panel (b), it is apparent that the model residual follows similarly to V14 (see their Figure 1) but there is less variability in the 2015 HWRF version as $\sigma_{m}$ is almost $1 \mathrm{~m} \mathrm{~s}^{-1}$ lower than their experimental HWRF results. The SFMR PDF, which is identical to V14, represents an uncertainty in the intensity estimate on the order of that determined from the best track. Comparing the SFMR result to the similarly produced scatterometer residual PDF, the average scatterometer residual is lower and is well within the expected uncertainty. However, the variability in the PDF indicates that 
the low wavenumber technique has conditions upon which it does not represent the best track intensity well. The larger variability compared to the best track data does not necessarily mean the scatterometer data are incorrect. Increased uncertainty in best track estimates along with uncertainty in the scatterometer analysis could both combine to cause the overestimation at weak wind speeds.
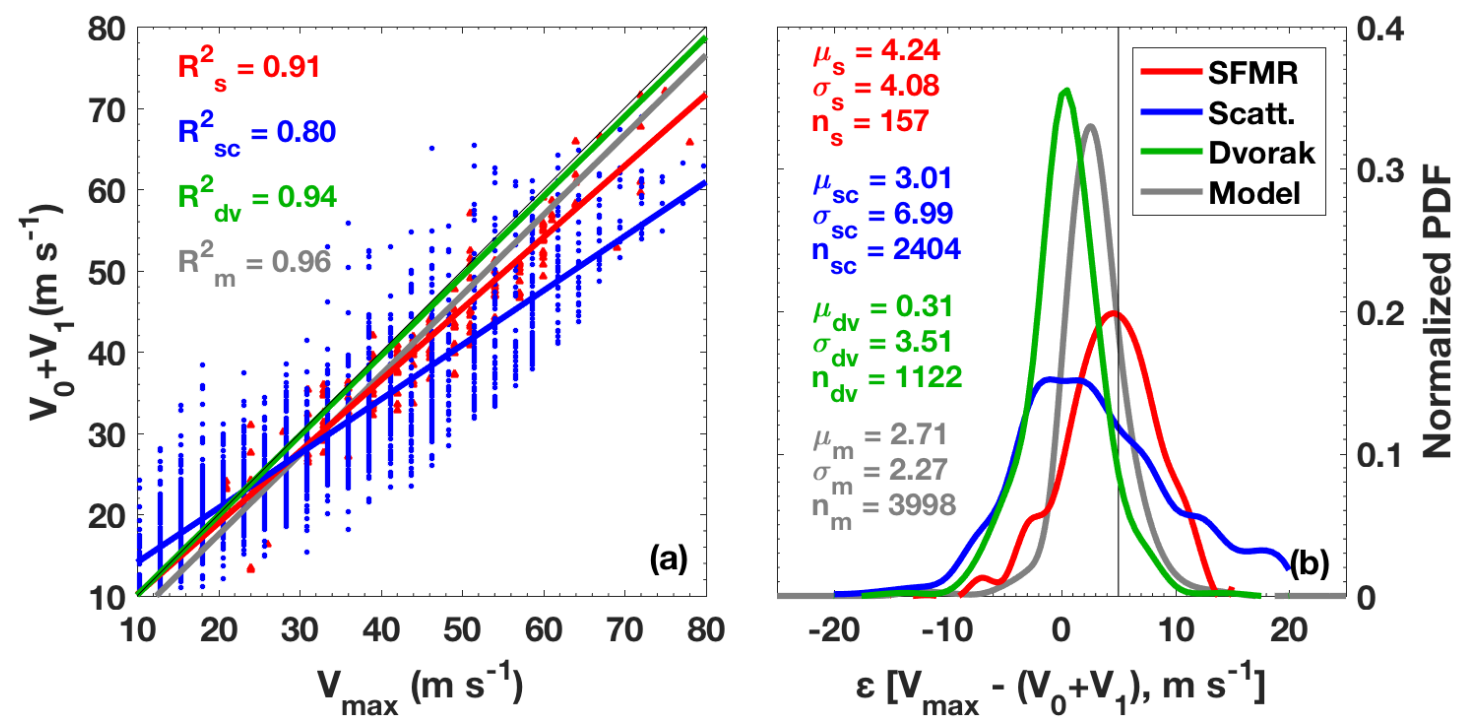

Figure 5.2. In (a), the paired samples of best track and scatterometer (blue), SFMR (red), and Dvorak (green) intensity estimates are provided. Model results (in gray) are evaluated against the model determined maximum $10-\mathrm{m}$ wind speed at a given forecast time. Solid lines indicate the linear regression fit of the data with coefficient of determination values indicated by matching colored text. In (b), the residual PDFs of the scatterometer, SFMR, and model low wavenumber data are provided. A PDF of the Dvorak differences is also shown but is not representative of the epsilon term. Mean $(\mu)$, standard deviation $(\sigma)$, and sample size $(n)$ are indicated as well as the average uncertainty from Landsea and Franklin (2013, black vertical line).

CDFs for these same samples in Figure 5.2 are shown in Figure 5.3. First notice that the model CDF has the least variability and indicates a less than $5 \%$ probability of having negative $\varepsilon$. The excellent agreement is expected considering the $V_{\max }$ and low wavenumber amplitude are derived from the same wind field. V14 notes it is possible to have negative $\varepsilon$ in certain cases due to higher wavenumber superposition, but the CDF 
indicates the chances of this are minimal for this HWRF dataset. The scatterometer distribution stands out the most, as its interquartile range is about twice as large as those from the other sources. The scatterometer result again confirms that there is higher variability in the low wavenumber amplitude from the scatterometer data when considering the full intensity spectrum. Compared to the SFMR and Dvorak distributions, which interestingly have similar slopes offset by approximately $5 \mathrm{~m} \mathrm{~s}^{-1}$, the likelihood of the scatterometer analyses providing reliable intensity is degraded. It is clear that the scatterometer analysis is outperformed by the SFMR (and Dvorak) overall, but the scatterometer is known to represent tropical storm winds well, leaving open the possibility of superior performance in this region of the wind speed spectrum.

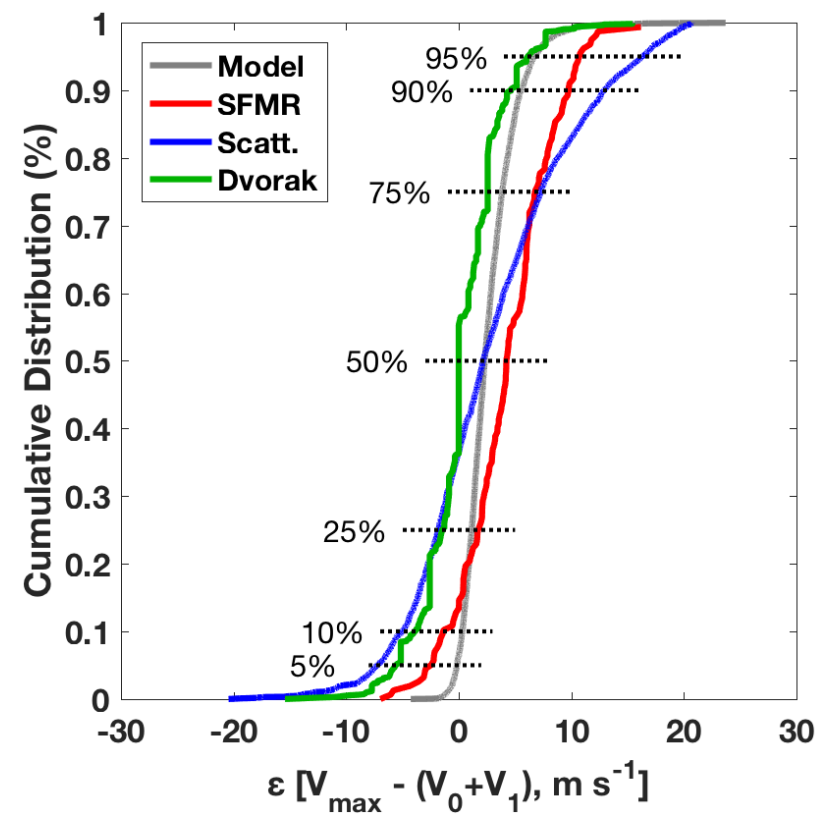

Figure 5.3. Cumulative distribution functions (CDFs) of $\varepsilon$ are provided for model, SFMR, and scatterometer data. The CDF for the Dvorak intensity difference is also included. Colors are the same as in Figure 5.2. 


\subsubsection{Tropical Cyclone Basin Dependency}

As noted in section 5.1, aircraft reconnaissance is rare in most of the world's TC basins. For this reason, Dvorak intensity estimates are one of if not the most reliable means of assigning TC intensity. Figure 5.4(a) provides basin-specific PDFs of intensity difference between the Dvorak estimates and their associated best track intensity. In theory, the basins with the least impact from aircraft reconnaissance should have better relationships between Dvorak and best track intensity estimates. However, the PDFs in Fig, 5.4(a) indicate that the WPAC and SHEM (no reconnaissance) are more variable than the EPCP and NATL (reconnaissance available). This conflict is present because the Dvorak technique struggles with storms within the tails of the intensity distribution. As indicated by Table A1.1 and Table 4.1, WPAC and SHEM basins contain a larger number of strong hurricanes and weak tropical storms and a larger number of RI cases compared to the other basins. Combined with the inherent uncertainty in the best track for satellite only estimates, these results confirm those of Knaff et al. (2010).
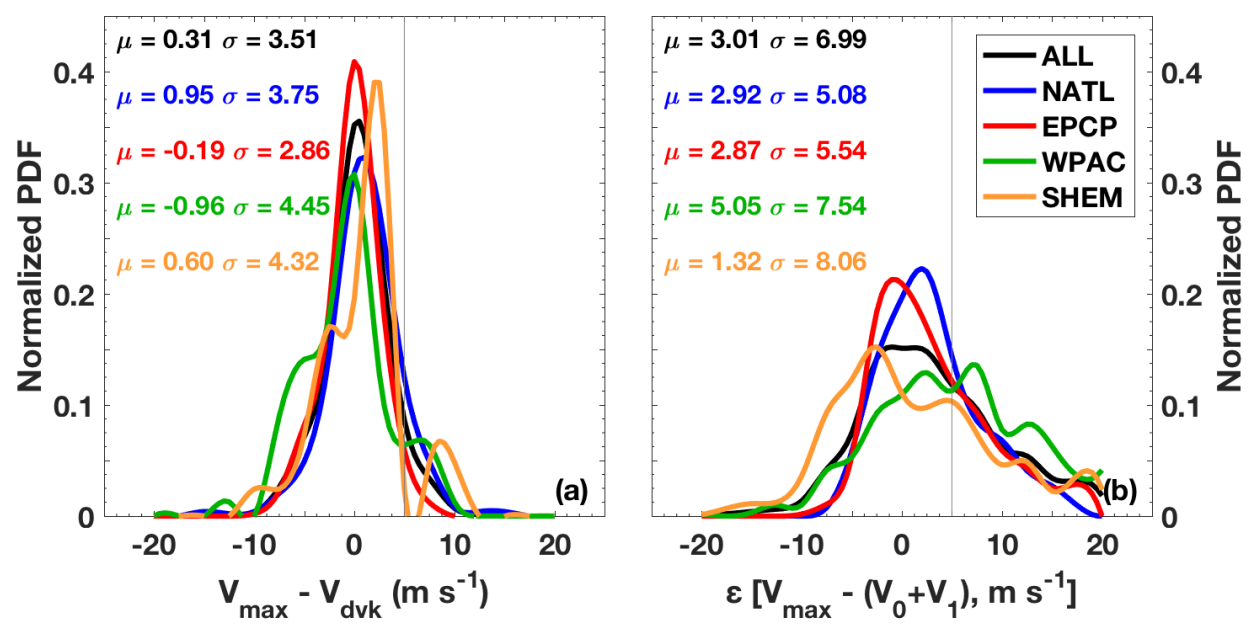

Figure 5.4. In (a), PDFs of the Dvorak difference with best track $V_{\max }$ are shown for all cases and for each basin. The matching colored text indicates mean and standard deviation. Similarly to (a), panel (b) provides PDFs of $\varepsilon$ for the scatterometer data. The black vertical line is representative of the best track intensity uncertainty. 
The general basin performance of the residual wavenumber term from the scatterometer analyses is provided in Figure 5.4(b). With the exception of the WPAC cases, all basins predict overall uncertainties lower than the expected best track uncertainty, where NATL and EPCP are most reliable due to their comparatively low mean and standard deviation values. SHEM cases have a low mean difference with the best track $V_{\max }$, but the $8 \mathrm{~m} \mathrm{~s}^{-1}$ standard deviation is concerning for using this verification metric in this basin. The inherent problem with evaluating the low wavenumber metric for WPAC and SHEM basins is that 1) the best track $V_{\max }$ here is more uncertain by $>2$ $\mathrm{m} \mathrm{s}^{-1}$ due to the fact that an already uncertain Dvorak estimate is a significant component of $V_{\max }$, and 2) the scatterometer low wavenumber analysis method is more uncertain for weak tropical storms and strong hurricanes. Therefore, the scatterometer analyses could be useful in these basins but only within the wind speed region where uncertainties in the Dvorak and low wavenumber $V_{\max }$ are minimized (between $\sim 20-40 \mathrm{~m} \mathrm{~s}^{-1}$ ).

\subsubsection{Tropical Cyclone Intensity}

To investigate whether the dependency on TC strength is true, PDFs of $\varepsilon$ similar to Figure 5.2 are displayed in Fig 5.5(a-c) for tropical storms, Category 1-2 hurricanes (non-major), and Category 3-5 hurricanes (major), respectively. The model derived residual term is not dependent on storm intensity as the mean and standard deviation differences are $\leq 0.5 \mathrm{~m} \mathrm{~s}^{-1}$ between the three wind speed regimes. The model result indicates that the low wavenumber technique from a model perspective is capable of producing reliable and verifiable intensity estimates for the entire intensity spectrum. With the low probability of having negative $\varepsilon$, superposition of high wavenumbers is also unlikely in the model evaluation. Table 5.3 provides the lowest $10 \%$, interquartile range 
(IQR), and 90\% value for the intensity dependent CDFs for the model, SFMR, and scatterometer. It also includes the same values for intensity change bins, which are discussed in a subsequent section.

Table 5.3. Provided are the $\varepsilon$ values taken from their CDFs and represent the lowest $10 \%$ (first sub-column), interquartile range (25-75\%, second sub-column), and highest $90 \%$ (third sub-column) for the model, SFMR, and scatterometer datasets. These values are stratified according to the defined TC intensity and intensity change groups.

\begin{tabular}{|c|c|c|c|c|c|c|c|c|c|}
\hline & \multicolumn{3}{|c|}{ Model } & \multicolumn{3}{c|}{ SFMR } & \multicolumn{3}{c|}{ Scatterometer } \\
\hline TS & 0.2 & $0.9-3.7$ & 5.7 & -3.8 & $-2.2-3.6$ & 9.9 & -6.4 & $-3.3-2.4$ & 5.3 \\
\hline CAT 1-2 & 0.4 & $1.3-3.9$ & 5.3 & -0.4 & $2.0-5.7$ & 7.7 & -1.7 & $2.9-10.3$ & 13.4 \\
\hline CAT 3-5 & 0.8 & $1.8-4.5$ & 5.8 & 0.5 & $4.1-8.3$ & 10.5 & 2.2 & $7.6-16.7$ & 18.9 \\
\hline \multicolumn{9}{|c|}{} \\
\hline RI & 0.0 & $1.4-3.7$ & 4.8 & -1.7 & $1.0-6.2$ & 9.7 & -5.9 & $-2.0-6.7$ & 10.2 \\
\hline SI & 0.2 & $1.2-3.5$ & 4.8 & -1.4 & $1.6-6.6$ & 8.3 & -4.8 & $-2.1-6.6$ & 12.3 \\
\hline SS & 0.4 & $1.2-3.8$ & 5.9 & -2.2 & $1.0-7.6$ & 10.3 & -5.9 & $-2.7-4.8$ & 10.6 \\
\hline WK & 0.3 & $1.1-4.2$ & 6.2 & 1.5 & $3.6-6.8$ & 8.8 & -1.3 & $2.9-12.5$ & 16.8 \\
\hline
\end{tabular}

The observation-based analyses indicate less certainty in terms of superior performance against the best track maximum. For tropical storms in Figure 5.5(a), the scatterometer and SFMR PDFs are surprisingly similar and indicate that the low wavenumber amplitude is in general agreement with the best track intensity. It is also indicative of the higher chance of overestimating the intensity in the TS wind regime. The IQR for the SFMR and scatterometer cases have a significant percentage of negative $\varepsilon(>25 \%)$, which for the SFMR could be due to its tendency to overestimate weak wind speeds (Uhlhorn et al. 2007). For the non-major hurricanes, the SFMR correctly represents a reasonable amount of uncertainty that would be accounted for by the remaining wavenumbers (confirmed by Table 5.3), but the scatterometer analyses begin to underestimate the best track intensity beyond the standard uncertainty. Note that the $6.17 \mathrm{~m} \mathrm{~s}^{-1}$ average difference is within the confidence interval of uncertainty (Landsea 
and Franklin 2013). This evidence indicates that the scatterometer is useful for non-major hurricanes but likely for case-by-case evaluations. Finally, major hurricanes are again well represented by the SFMR data despite the $>5 \mathrm{~m} \mathrm{~s}^{-1}$ peak probability. Uncertainties in the best track $V_{\max }$ within major hurricane winds can be as high as $7 \mathrm{~m} \mathrm{~s}^{-1}$ on average. The scatterometer PDF (statistically significant at 95\%) indicates there is no predictive power for the low wavenumber technique for major hurricanes and that verification for models against the best track value is preferable in the absence of aircraft data. Therefore, the scatterometer low wavenumber $V_{\max }$ is a useful metric for model verification for TCs less than major hurricane strength with preference for tropical storms or Category 1 hurricanes.
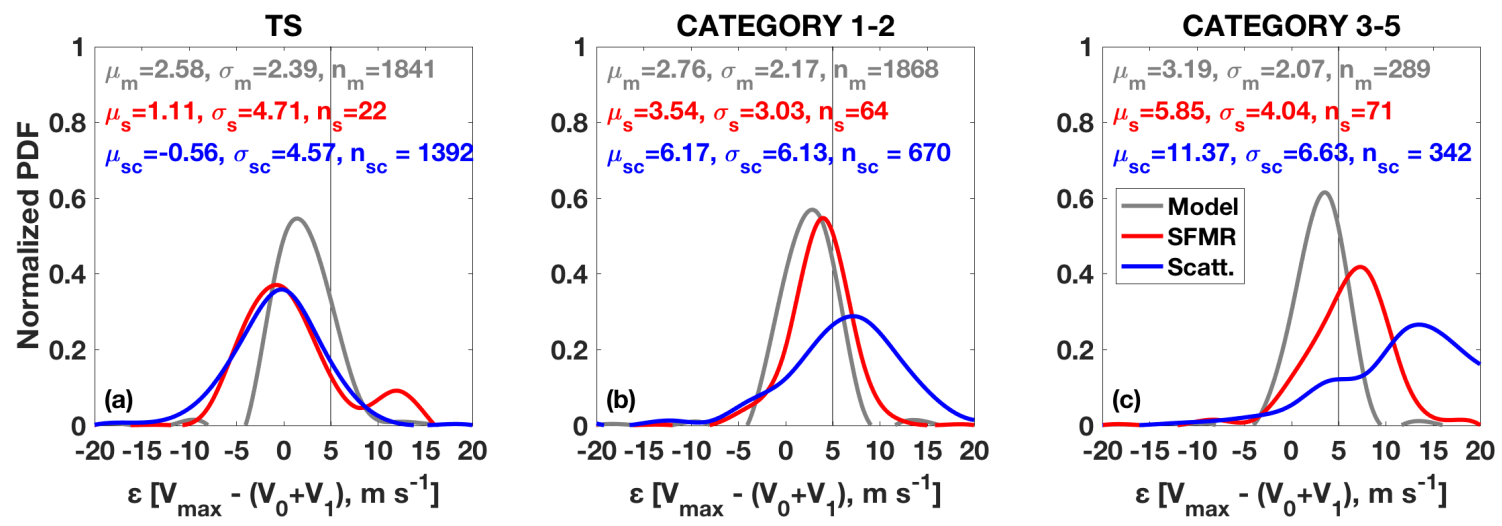

Figure 5.5. Similarly to Figure 5.4(b), PDFs of $\varepsilon$ are separated by storm intensity for (a) tropical storms, (b) Category 1-2 hurricanes, and (c) Category 3-5 hurricanes and are provided for model (gray), SFMR (red), and scatterometer (blue) data. Mean, standard deviation, and sample size are indicated by the text.

\subsubsection{Tropical Cyclone Intensity Change}

As determined in the structural analysis of the scatterometer winds, distinguishable asymmetry characteristics were most noticeable as a function of TC intensity. The previous section also confirms that the $V_{0+1, \max }$ estimate from the scatterometer analysis is dependent on TC intensity. Changes in TC strength, especially 
those that occur quickly (i.e., rapid intensification or weakening), are troublesome for forecasters (Elsberry et al. 2007) and for TC models (Davis et al. 2006; Pu et al. 2009; Kaplan et al. 2010). These intensity change errors pose a problem for verification purposes as the uncertainty of the associated $V_{\max }$ increases too.

In order to test the reliability of the low wavenumber method for model verification, PDFs of $\varepsilon$ are provided for four intensity change groups (RI, SI, SS, and WK) in Figure 5.6. As with the TC intensity stratification, model PDFs of $\varepsilon$ are almost unaffected by the rate of intensity change with mean (standard deviation) values between 2 and $3 \mathrm{~m} \mathrm{~s}^{-1}\left(1.7\right.$ and $\left.2.7 \mathrm{~m} \mathrm{~s}^{-1}\right)$. Despite the small sample (1\% of the total), the representative high order wavenumbers for the RI cases are similar to the other three groups. It is unclear if this is due to consistency within the model data, but it is suspected that the agreement between the stratifications is a result of the initial intensity of the modeled TC. Discussion in Chapter 4 revealed that the scatterometer analyses were most sensitive to intensity rather than intensity change, and the same appears to be true for the model uncertainty. Considering that a higher percentage of WK cases are at least moderately strong hurricanes ( $\geq$ Category 2 ), this suspicion is supported by the slightly higher model uncertainty.

The hypothesis above holds true for the SFMR and scatterometer PDFs as both exhibit their largest average $\varepsilon$ for WK cases. Interestingly, the standard deviation for the SFMR cases here is the lowest of the four groups, which could be simply due to low variability within the small sample (IQR is the lowest as well). The scatterometer PDF here also resembles a blend of the two hurricane groups in Figure 5.5, where the majority of the high probabilities are greater than the expected uncertainty. With more than $10 \%$ 
of these cases having $\varepsilon$ below the uncertainty threshold, it could be useful to use the scatterometer analysis for verification as long as the initial intensity is $\leq 50 \mathrm{~m} \mathrm{~s}^{-1}$. V14 suggests that for the SFMR, the low wavenumber technique is designed to not overestimate the uncertainty, which is the reasoning behind much lower probabilities associated with negative $\varepsilon$ compared to the scatterometer.
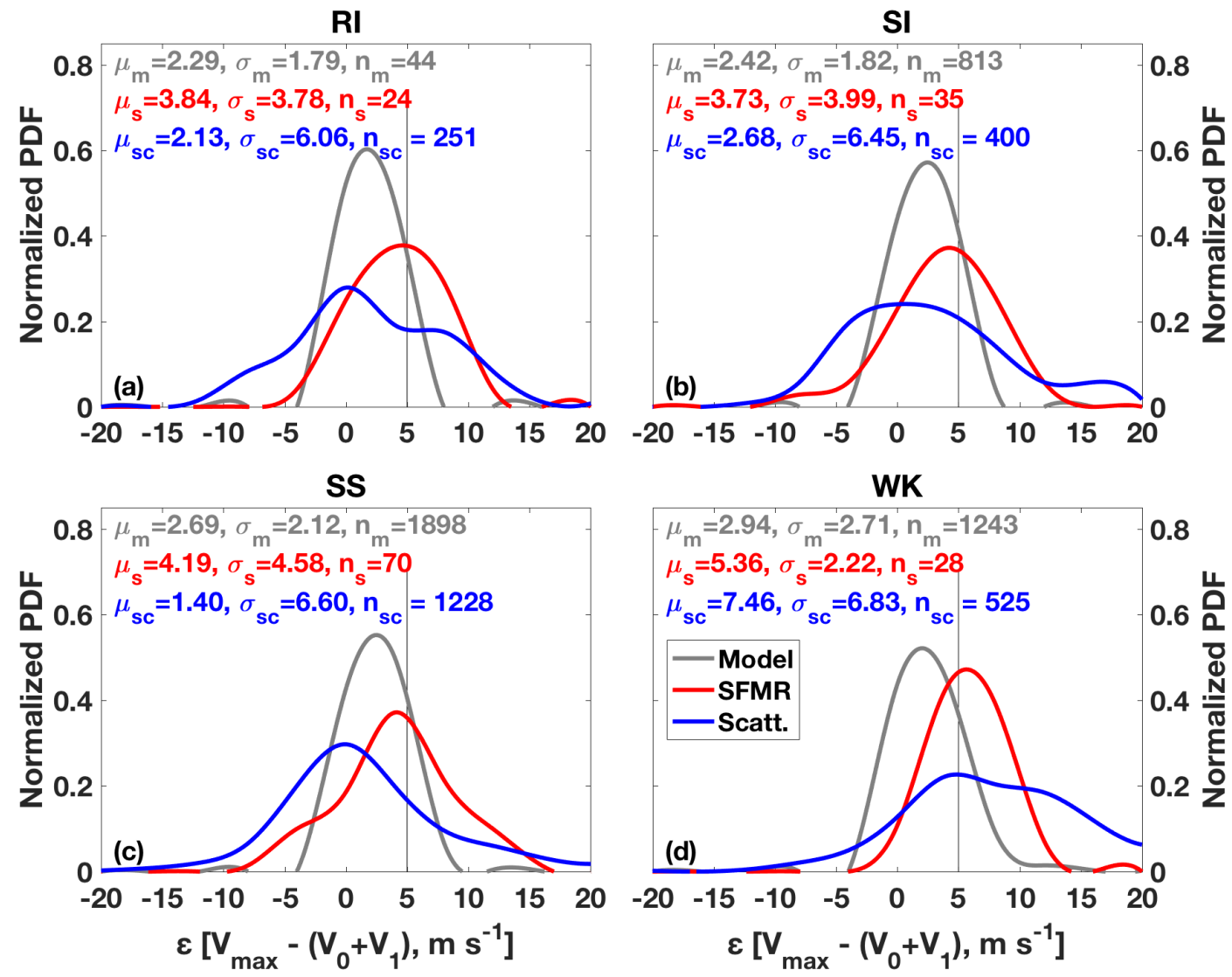

Figure 5.6. Similar to Figure 5.5, PDFs of $\varepsilon$ are separated by intensity change groups for (a) rapidly intensifying, (b) slowly intensifying, (c) steady state, and (d) weakening TCs.

For the non-weakening cases, the CDF values in Table 5.3 indicate that there is not a significant difference between the expected residual within each observation dataset. The result here confirms that initial TC intensity rather than a change in the intensity is likely playing a role in the magnitude of the uncertainty. Therefore, the main conclusion deduced from the results in this and the previous sections is that the 
scatterometer data is an alternative metric that can be used to verify model intensity forecasts in the absence of aircraft data and should mainly be trusted for tropical storms and non-major hurricanes due to the long list of possible errors at the edges of the wind spectrum.

5.5 Effectiveness of Using Scatterometer Data for Tropical Cyclone Intensity Verification

From the results and discussion in V14 and in the previous section, the SFMR and scatterometer low wavenumber analyses are valid alternatives to verifying model intensity metrics under most circumstances. The next task is to examine the mean and mean absolute errors between the scatterometer and model low wavenumber parameters $\left(V_{0}, V_{1}, V_{0+1}, \varepsilon\right.$, and $\left.V_{\max }\right)$ as a function of forecast hour. Figures 5.7 and 5.8 provide examples of the comparison between the low wavenumber variables for three successive simulations in Hurricane Irwin (11E) and Hurricane Katia (12L), respectively. Note that neither case had aircraft reconnaissance within 48 hours of the model initial time displayed.

According to Berg (2012), Irwin developed as a disturbance from the Intertropical Convergence Zone (ITCZ) and rapidly intensified from a tropical depression to Category 1 hurricane during the period of the three simulations. The best track $V_{\max }$ (solid blue line) is initially significantly higher than the model equivalent by nearly $10 \mathrm{~m} \mathrm{~s}^{-1}$ (forecast time 24 hours) in Figure 5.7(a). However, the $V_{0+1, \max }$ term for the model (gray solid line) and scatterometer (red solid line) are within 2-3 $\mathrm{m} \mathrm{s}^{-1}$ of each other at this same forecast time. The best track values in all three simulations indicate a rapid weakening period early in the forecast period, which according to Berg (2012) was due to interaction with a 
trough and outflow from Hurricane Jova. However, the combined wavenumber amplitudes for the model and scatterometer suggest a slower rate of weakening, and the difference between these two values increases significantly during the weakening period. During the steady period after 72 hours, the amplitudes verify again as they are almost on top of each other for the first simulation. The two additional model runs indicate a higher intensity beyond three days compared to the best track, and the scatterometer is less in agreement here.

IRWIN (11E) 2011100618

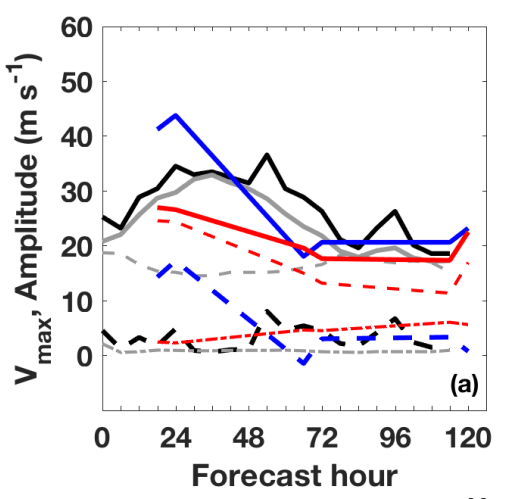

IRWIN (11E) 2011100700

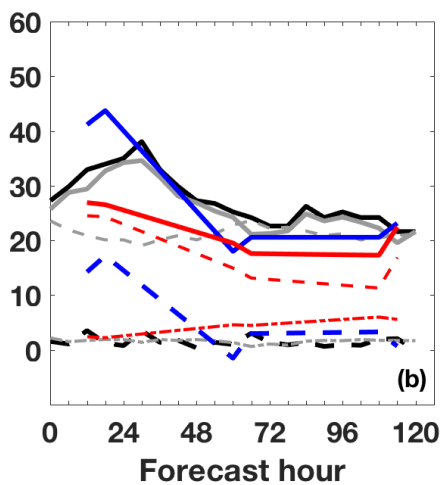

IRWIN (11E) 2011100706

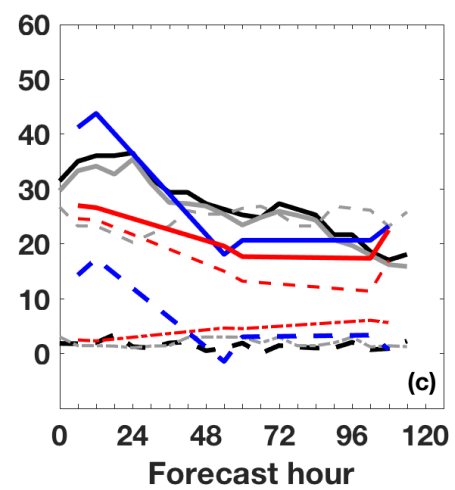

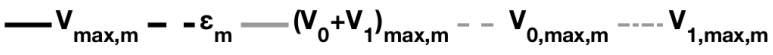

$-v_{\text {max }, s c}--\varepsilon_{s c}-\left(V_{0}+V_{1}\right)_{\max , s c}--v_{0, \text { max sc }}---v_{1, \text { max }, s c}$

Figure 5.7. Three successive HWRF simulations Hurricane Irwin (11E) are shown and include the best track $V_{\max }$ (solid blue line), model $\mathrm{V}_{\max }$ and $\varepsilon$ (black solid and dashed lines, respectively) and scatterometer $\varepsilon$ (blue dashed line) as a function of forecast hour. The combined $\left(V_{0+1, \max }\right)$ and individual low wavenumber amplitudes $\left(V_{0, \max }\right.$ and $\left.V_{1, \max }\right)$ are provided for model (gray solid, dashed, and dash-dot lines respectively) and scatterometer (red solid, dashed, and dash-dot lines) data.

In the Irwin example, it is evident that $V_{1}$ is always larger for the scatterometer than for the model analysis, suggesting a systematic difference between the model and scatterometer analyses. Additionally, the model and scatterometer $V_{0+1}$ do not capture the rapid intensification or the rapid weakening for any simulation, which for the scatterometer is due to the much weaker initial intensity. These comparisons indicate that 
the extremes of the intensity change spectrum are difficult to reproduce with the low wavenumber technique, but the model and observational products follow similar trends.

The Hurricane Katia example in Figure 5.8 showcases several conditions, including an intensification period in the first simulation that is captured in the best track, model, and scatterometer data. Katia developed from an easterly wave over the open Atlantic and steadily intensified for several days due to decreasing vertical wind shear (Stewart 2012). The period displayed in the simulations was within an ongoing RI event as the TC moved to the northwest. In the first simulation, the wavenumber amplitudes are in good agreement $\left(<5 \mathrm{~m} \mathrm{~s}^{-1}\right.$ difference) through most of the forecast period. For the second simulation, the model results are significantly different than in panel (a) and match closely to the best track $V_{\max }$. Because the model amplitude and best track $V_{\max }$ both indicate a major hurricane, it is not surprising that the scatterometer amplitude does not verify the model results. After the TC weakens beyond 48 hours, the three intensity metrics are within 2-3 $\mathrm{m} \mathrm{s}^{-1}$ of each other. Similar trends are seen in the third simulation in panel (c). Katia provides another example that the uncertainty in the scatterometer analysis prevents its consistent ability to verify model intensities, especially for strong hurricanes and rapidly intensifying periods. 
KATIA (12L) 2011090406

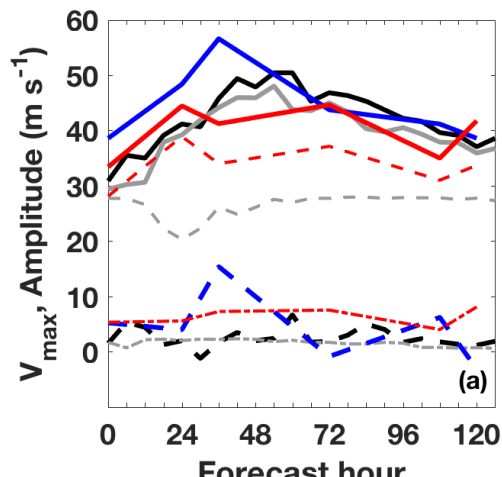

KATIA (12L) 2011090412

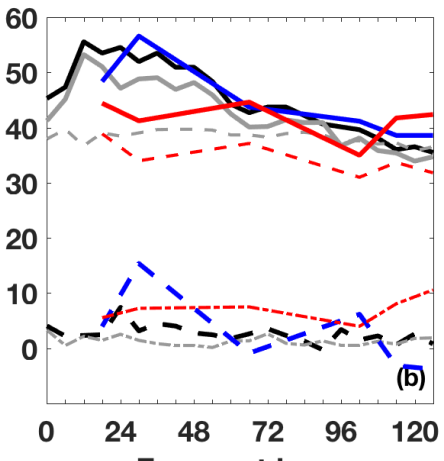

KATIA (12L) 2011090418

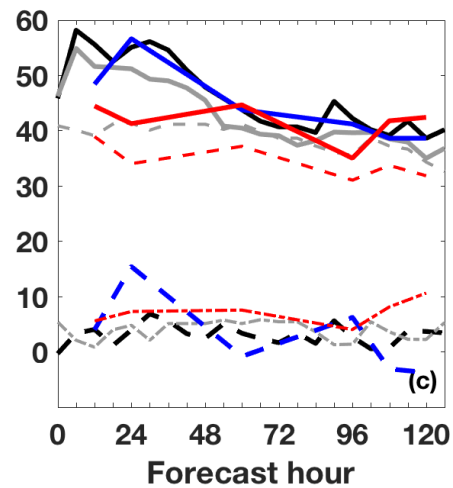

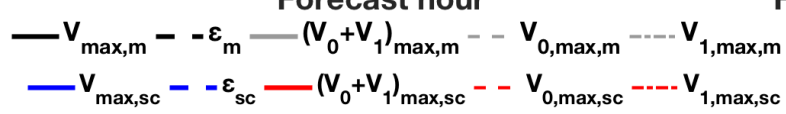

Figure 5.8. Similar to Figure 5.7. but for Hurricane Katia (12L).

The individual cases are examples of the advantages and disadvantages of using the scatterometer to verify the model intensity metric. To understand the overall skill of the scatterometer technique relative to the best track verification metrics, Figure 5.9 displays the mean and mean absolute errors (MAEs) in (a) and (b), respectively, as a function of forecast hour. These differences follow the constraints exemplified in Eq. 5.1a and b. Note that only cases with $V_{\max }<50 \mathrm{~m} \mathrm{~s}^{-1}$ were included in this examination to eliminate the large scatterometer underestimate at major hurricane strength. The mean errors associated with the $V_{0}$ and $V_{\max }$ differences (blue and purple lines) follow similar trends due to the fact that $V_{0}$ is the dominant component of the low wavenumber analyses. Errors in the forecast and in the observational data are closely tied to variations in this axisymmetric term. In other words, uncertainty in the axisymmetric surface winds generally defines the metric's ability to successfully verify the model intensity. The large underestimate seen in the major hurricanes in Figure 5.2 and 5.3 is explained by this uncertainty because the scatterometer winds are increasingly uncertain above $50-55 \mathrm{~m} \mathrm{~s}^{-1}$ (Stiles et al. 2014). 
The $V_{I}$ mean error (red line) in Figure 5.9(a) is always negative and becomes more negative with increasing forecast time. The $V_{I}$ trend compensates the increasing $V_{0}$ errors and is partly the cause of the near zero errors of $V_{\max }$. Beyond approximately 60 hours, $V_{0}$ increases more quickly, and the resulting $V_{\max }$ from the model begins to overestimate the observed $V_{\max }$. Because the residual term (green line) is also negative through 48-60 hours, the increased difference in uncertainty accounts for some portion of the larger $V_{\max }$ representation by the scatterometer. It is clear that the mean errors are dominated by the axisymmetric winds but these errors are somewhat compensated by larger scatterometer $V_{l}$ and residual uncertainties through 60 hours.
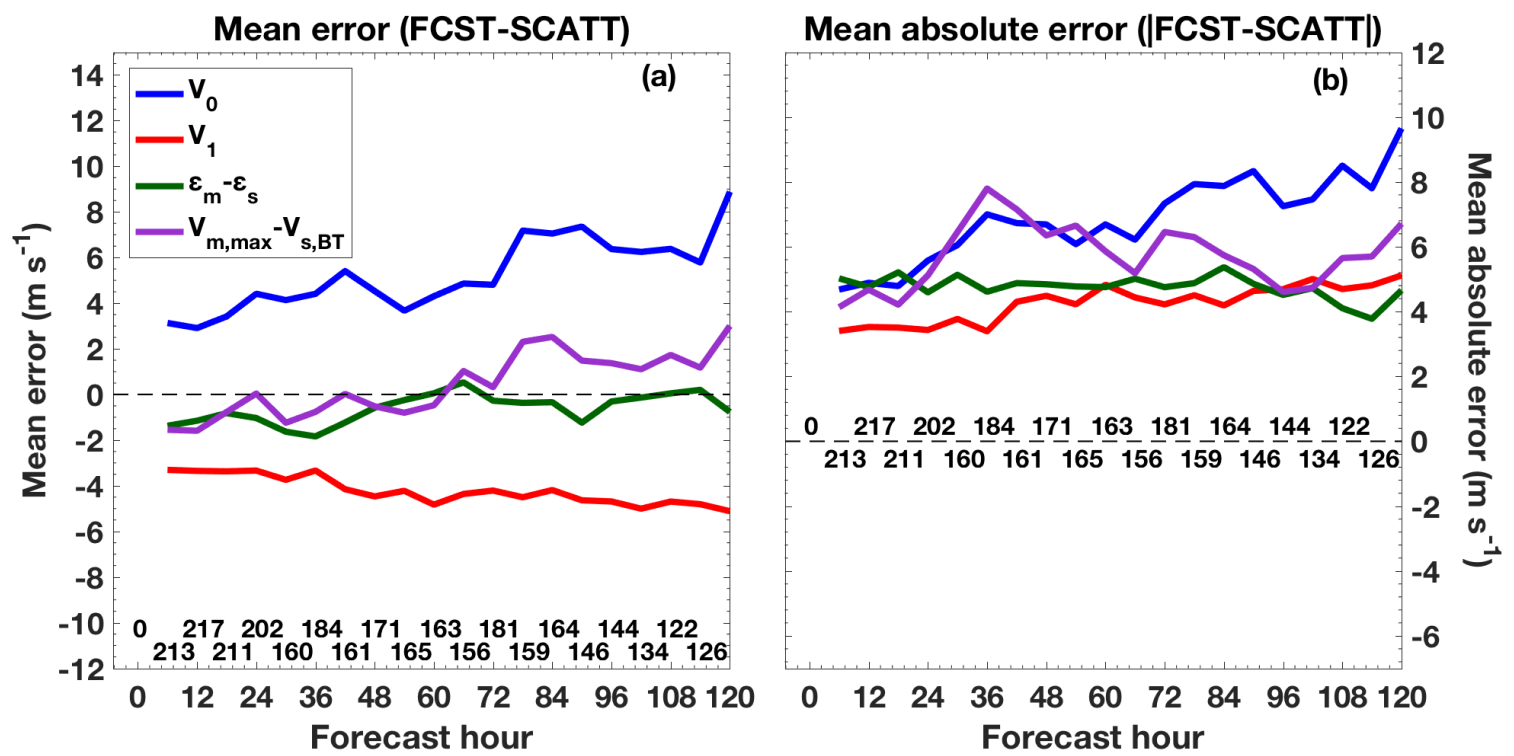

Figure 5.9. In (a), mean error of the forecast value compared to the same variable from the scatterometer data is shown as a function of forecast hour for $V_{0}$ (blue), $V_{l}$ (red), $\varepsilon$ (green), and $V_{\max }$ (purple). The number of matching forecast cases is provided at each 6$\mathrm{hr}$ forecast time. In (b), similar lines are provided as in (a) but for the mean absolute error. The cases considered in these comparisons include only TCs below major hurricane strength.

Similar compensation is noticed in the MAEs provided in Figure 5.9(b) for the first 60 forecast hours. The two intensity metrics are generally in line with each other and 
show MAEs between 4 and $7 \mathrm{~m} \mathrm{~s}^{-1}$, indicating that the scatterometer has skill for verification of the model equivalent terms within the first 2.5 days of a TC simulation. Beyond 60 hours, MAEs of $V_{0}$ increase while the best track metric remains nearly constant. Residual and asymmetric amplitudes also remain relatively constant here $\left(V_{I}\right.$ increases by 1-1.5 $\mathrm{m} \mathrm{s}^{-1}$ over the full forecast) and indicate these terms are less important at extended forecast times. The result here could be explained by the fact that $V_{I}$ constitutes less of the $V_{0+1}$ amplitude as the intensity of a TC increases (see the Irwin and Katia examples). Considering that the average model and best track $V_{\max }$ increases with forecast time ( $\sim 8$ and $4 \mathrm{~m} \mathrm{~s}^{-1}$, respectively, between initial and final times), it is not surprising that the best track metric is more reliable at later times. Based on these error estimates, the scatterometer low wavenumber metric generally has equivalent to better skill compared against the best track for the first 60 hours while the best track skill exceeds the scatterometer beyond day three.

5.6 Preliminary Analysis of Rain-Relative Wind Structure

The scatterometer low wavenumber technique for model verification has been proven to represent the residual uncertainty in most conditions and has verification skill through 2-2.5 days in a given forecast period. These verification metrics evaluated the intensity estimate from model forecasts. However, the Diapost post-processed files also enable the verification of surface wind structure characteristics as presented in Chapters 3 and 4 . The most beneficial advantage of the model is the coincident rain and surface wind parameters that are rarely observed together in nature. Surface winds are the key reference here as wind and rain parameters are collected in tandem during NOAA reconnaissance flights (Rogers et al. 2013), but relationships are generally related to 
flight-level winds due to the limitations of the low-level Doppler radar derived winds (historically $0.5 \mathrm{~km}$ vertical resolution with lowest level of $0.5 \mathrm{~km}$, Gamache et al. 1995).

Ueno and Kunii (2009) describe a theoretical relationship between upward motion and the location of the surface maximum winds, suggesting that the upward motion (convective proxy) is $\sim 90^{\circ}$ upwind of the wind maximum in a motion-relative sense. Understanding that the rain would precipitate downwind of this location, the maximum rain would generally occur between $0-90^{\circ}$ upwind of the surface wind maximum. The relationship between rain and wind structure is not really consequential in terms of TC intensity, but it could be useful for interpreting intensity change conditions. In Chapter 4, a rain rate maximum was compared to the azimuthal difference with the wavenumber-1 phase during a composite RI event, and it is apparent that prior to and during RI, the phase relationship is in agreement with theoretical discussions. By the end of the RI event, the rain rate maximum azimuth is located coincident with or slightly downwind of the wind maximum.

Using the model output, it is possible to verify whether this relationship for RI conditions is unique. Figure 5.10 provides a normalized, joint PDF of the intensity change groups and of the azimuthal difference between the maximum rain rate and maximum surface wind speed. Following the trend line associated with the maximum probability (black line), arguably the maximum wind and rain parameters are more likely to align azimuthally for the weakening cases ( $\mathrm{SW}$ and $\mathrm{RW}$, which is defined as a 24-hour $V_{\max }$ decrease of more than $30 \mathrm{kt}$ ). The difference between these and the other three groups is statistically significant at $95 \%$. For the non-weakening cases, there is less clarity as $\mathrm{SS}$ and RI cases tend to experience maximum rain rates $\sim 90^{\circ}$ upwind of the 
surface wind maximum. Note the nomenclature in reference to upwind and downwind differences are forward and rear (DWF and DWR, respectively, for example), where $\Delta \phi$ designated as DWF represents a wavenumber- 1 phase between $180^{\circ}$ and $90^{\circ}$ downwind of the rain maximum (negative values).

From Figure 5.10, the general trend of the intensity change PDFs (rows) is to move from an upwind maximum rain location to a coincident or slightly downwind location from RI to RW, which tends to agree with the observational results. This trend is due to the alignment of the shear and motion vectors, as WK TCs (especially RW) move poleward quickly and are generally under the influence of high shear. Convective processes begin to get cutoff at this stage as outflow aloft and low level moisture inflow are slowed. With no mechanism for maintaining its strength, the TC is either converted to an extratropical system or dissipates. Therefore, the model evaluations confirm the observational results to an extent, and there is predictive skill here as conditions where the maximum rain rate and surface wind speed align will inhibit intensification.

A relationship between the phase of the low wavenumber analysis and maximum rain rate was determined from observations (see Chapter 4) for various stages of an RI event. Following similar methods, a normalized, bivariate PDF of the model-derived relationship is provided in Figure 5.11 for the same RI periods. For reference, $\mathrm{RI}_{0}$ and $\mathrm{RI}_{\mathrm{E}}$ denote the initial and ending time of the $\mathrm{RI}$ event, respectively. Any values associated with these times indicate the number of hours relative to either the RI initial or RI ending time. Of the 554 model simulations (including ones without overlapping scatterometer data), $\sim 12 \%$ experienced an RI event. These events last between 30 and 48 hours, averaging a length of 36 hours overall. 


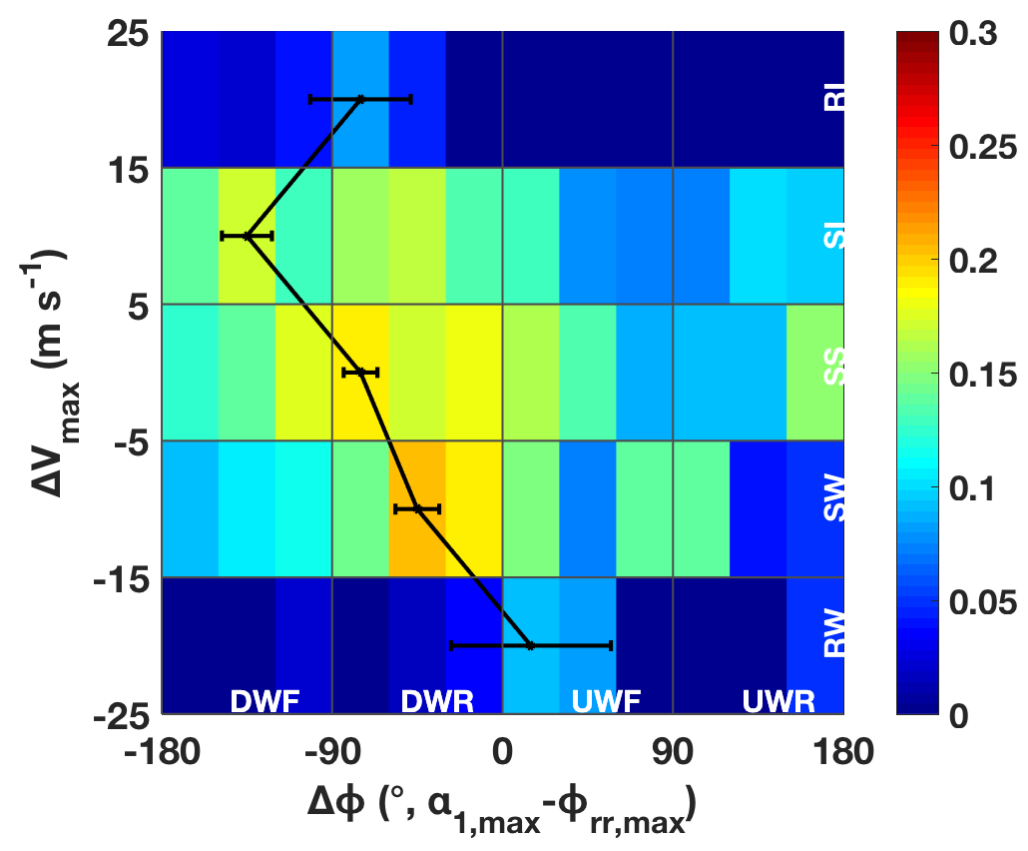

Figure 5.10. Displayed is a normalized joint PDF of the rate of intensity change and the wavenumber-1 phase difference with the azimuthal location of the maximum 10-m rain rate as determined from the model forecast data $(\Delta \phi)$. Warmer colors indicate higher probabilities and the black markers represent the azimuthal difference bin with the highest probability for each intensity change bin. The horizontal error bars indicate the $95 \%$ confidence interval. Negative angles indicate that $\alpha_{1, \max }$ is downwind of $\phi_{r, \max }$, where DWF and DWR refer to the downwind forward and rear quadrants.

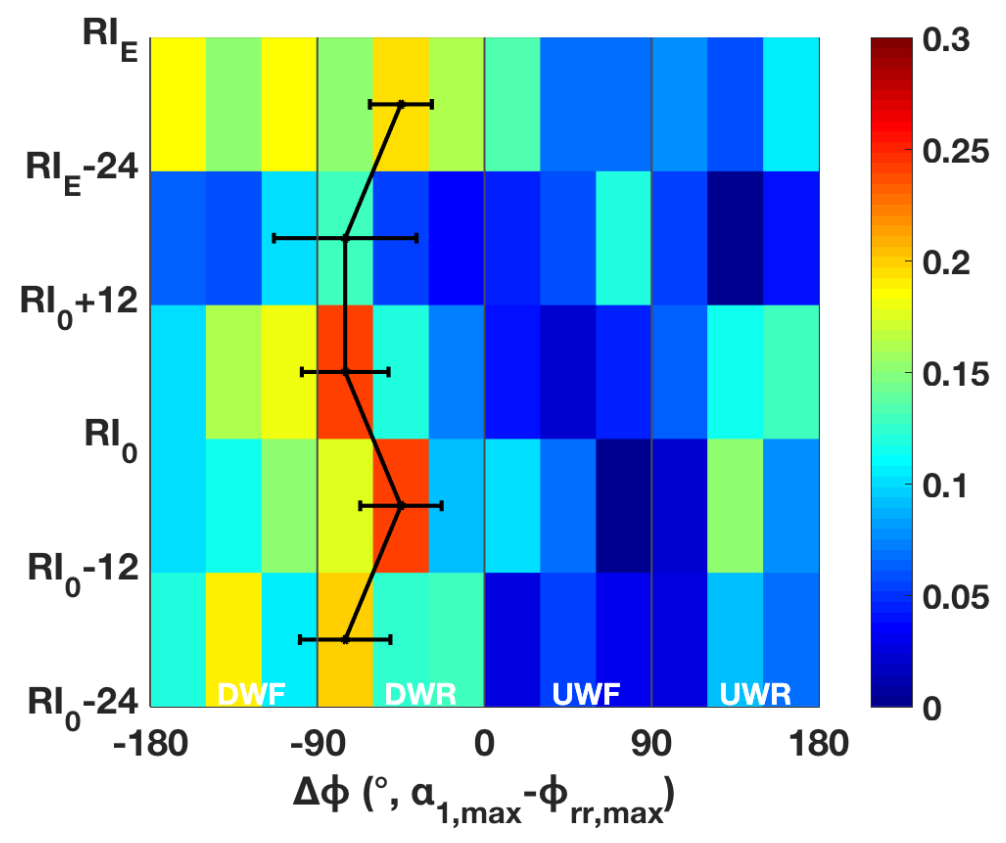

Figure 5.11 Similar to Fig 5.10 but for the designated RI event time periods. $\mathrm{RI}_{0}$ and $\mathrm{RI}_{\mathrm{E}}$ indicate RI initial and ending times, respectively. 
The maximum probability remains consistent through the RI event, indicating that the maximum low wavenumber amplitude is between $60-90^{\circ}$ downwind of the maximum rain rate. Note the PDFs of each row, however, indicate a progression of higher probabilities DWF to DWR through the $\mathrm{RI}_{0}+12$ time. During the $\mathrm{RI}$ ending period, the probabilities are more variable than prior times but also indicate a greater likelihood of DWR or even coincident maximum wind amplitude relative to the rain rate. The eventbased results provide confirmation of the observational results by suggesting that the azimuthal difference between wind and rain locations is maximized prior to RI initiation.

During RI, they are closer to $60-90^{\circ}$ out of phase before becoming more aligned during the RI ending and post RI period.

\subsection{Summary and Conclusions}

In previous chapters, discussion of the observation-based surface wind asymmetry indicated dependency on wind shear by basin, storm intensity, and intensity change stratifications. The purpose of this chapter was to determine if the analyses used to evaluate the surface wind structure has predictive skill as an intensity metric. Using the low wavenumber amplitude $\left(V_{0}+V_{l}\right)$ and residual $\operatorname{PDF}(\varepsilon)$, comparisons against the standard best track and Dvorak intensity estimates were performed for the respective datasets as a whole. Further separation by the basin, intensity, and intensity change designations was performed on the low wavenumber metrics only (i.e. no Dvorak) due to increased uncertainty and existing discussion in Knaff et al. (2010). Skill of the model verification relative to the best track metric was then examined as a function of forecast hour in terms of the mean errors and MAEs. Evaluation of the difference between the model and observation low wavenumber parameters indicated that the scatterometer has 
predictive skill but only under non-major hurricane and tropical storm conditions and within an early forecast time periods. A final discussion of the predictability of surface wind structure relative to raining processes indicated that they would be most useful for separating weakening from steady-state or intensifying TCs.

On the basis of the results obtained from these analyses, the main conclusions are as follows:

- As a result of increased uncertainty in the low wavenumber amplitude compared to the best track for intense TCs, scatterometer estimates are only valid for tropical storms and non-major hurricanes. Residual PDFs reveal that the estimate of the higher order wavenumbers is within the expected best track uncertainty.

- Evaluation against the Dvorak intensity estimate for the different TC basins indicates that the scatterometer metric is useful for the NATL and EPCP basins but is more uncertain in the WPAC and SHEM due to increased uncertainty of the Dvorak estimate.

- Mean absolute errors produced from the difference of best track and model information indicate that the scatterometer metric has verification skill through 60 hours but performs worse than the best track metric at extended forecast times due to a higher intensity estimate.

- Low wavenumber structure derived from model data confirms theoretical expectations of the maximum wind relation to rain rate for intensity change groups and also indicates predictive skill for non-intensifying TCs. Azimuthal alignment of the maximum rain rate and surface wind is predictive of a $\mathrm{TC}$ that will likely not 
intensify. Evaluation with respect to RI event periods verifies the results determined from observational analysis.

Scatterometers are well-equipped to provide predictive skill against the best track in most conditions for model verification. However, the extent of the structural examination from the model wind fields was not exhaustively discussed. Components such as vertical motion or thermodynamic quantities could be used in a future study to determine the validity of the surface wind analyses but also could provide a better understanding of how the various processes are related to each other in terms of TC development and intensification. The preliminary examination here reveals there is a connection between these processes and has predictive capabilities. 


\section{REFERENCES}

Aberson, S. D., M. L. Black, R. A. Black, J. J. Cione, C. W. Landsea, F. D. Marks, and R. W. Burpee, 2006: Thirty years of tropical cyclone research with the NOAA P-3 aircraft. Bull. Amer. Meteor. Soc., 87, 1039-1055.

Aksoy, A., S. Lorsolo, T. Vukicevic, K. J. Sellwood, S. D. Aberson, and F. Zhang, 2012: The HWRF Hurricane Ensemble Data Assimilation System (HEDAS) for high-resolution data: The impact of airborne Doppler radar observations in an OSSE. Mon. Wea. Rev., $140,1843-1862$.

Aligo, E., Ferrier, B., J. Carley, E. Rodgers, M. Pyle, S. J. Weiss, and I. L. Jirak, 2014: Modified microphysics for use in high resolution NAM forecasts. 27th AMS Conference on Severe Local Storms. 3-7 November, Madison, WI.

Alvey III, G., J. Zawislak, and E. Zipser, 2015: Precipitation properties observed during tropical cyclone intensity change. Mon. Wea. Rev., 143, 4476-4492.

Bao, J.-W., S. G. Gopalakrishnan, S. A. Michelson, F. D. Marks, and M. T. Montgomery, 2012: Impact of physics representation in the HWRFX model on simulated hurricane structure and wind-pressure relationships. Mon. Wea. Rev., 140, 3278-3299.

Bender, M., I. Ginis, R. Tuleya, B. Thomas, and T. Marchok, 2007: The Operational GFDL coupled hurricane-ocean prediction system and a summary of its performance. Mon. Wea. Rev., 135, 3965-3989.

Berg, R., 2012: Tropical cyclone report: Hurricane Irwin. National Hurricane Center Tropical Cyclone Reports, [Available at http://www.nhc.noaa.gov/data/tcr/].

Bessho, K., M. DeMaria, and J. A. Knaff, 2006: Tropical cyclone wind retrievals from the Advanced Microwave Sounder Unit (AMSU): Application to surface wind analysis. J. Appl. Meteor. Climatol., 45, 399-415.

Black, M. L., R. W. Burpee, and F. D. Marks Jr., 1997: The asymmetric distribution of vertical motions and precipitation in the hurricane eyewall. Preprints, 22nd Conf. on Hurricanes and Tropical Meteorology, Fort Collins, CO, Amer. Meteor. Soc., 100-101.

Black, M. L., J. F. Gamache, F. D. Marks Jr., C. E. Samsury, and H. E. Willoughby, 2002: Eastern Pacific Hurricanes Jimena of 1991 and Olivia of 1994: The effect of vertical shear on structure and intensity. Mon. Wea. Rev., 130, 2291-2312.

Brand, S., C. A. Buenafe, and H. D. Hamilton, 1981: Comparison of tropical cyclone motion and environmental steering. Mon. Wea. Rev., 109, 908-909. 
Brennan, M. J., C. C. Hennon, and R. D. Knabb, 2009: The operational use of QuikSCAT ocean surface vector winds at the National Hurricane Center. Wea. Forecasting, 24, 621645 .

Bu, Y., 2015: Influence of cloud-radiative forcing and the planetary boundary layer on tropical cyclone structure, $\mathrm{PhD}$ Thesis submitted to the University of California Los Angeles.

Burpee, R. W., 1972: The origin and structure of easterly waves in the lower troposphere of North Africa. J. Atmos. Sci., 29, 77-90.

Burpee, R. W., and M. L. Black, 1989: Temporal and spatial variations of rainfall near the centers of two tropical cyclones. Mon. Wea. Rev., 117, 2204-2218.

Cangialosi, J., T. Kimberlain, J. Beven, and M. DeMaria, 2015: The validity of Dvorak intensity change constraints for tropical cyclones. Wea. Forecast., 30, 1010-1015.

Carrier, G. F., 1971: The intensification of hurricanes. J. Fluid Mech., 49, 145-148.

Cecil, D. J., 2007: Satellite-derived rain rates in vertically sheared tropical cyclones, Geophys. Res. Lett., 34, L02811.

Chan, J. C.-L., and R. T. Williams, 1987: Analytical and numerical studies of the betaeffect in tropical cyclone motion. Part I: Zero mean flow. J. Atmos. Sci., 44, 1257-1265.

Chan, J. C.-L., and J. Shi, 1996: Long-term trends and interannual variability in tropical cyclone activity over the western North Pacific. Geophys. Res. Lett., 23, 2765-2767.

Chan, K. T.-F. and Chan, J. C.-L., 2012: Size and strength of tropical cyclones as inferred from QuikSCAT data. Mon. Wea. Rev., 140, 811-824.

Chan, K. T.-F. and Chan, J. C.-L., 2013: Angular momentum transports and synoptic flow patterns associated with tropical cyclone size change. Mon. Wea. Rev., 141, 39854007.

Chan, K. T.-F. and J. C.-L. Chan, 2015: Global climatology of tropical cyclone size as inferred from QuikSCAT data. Int. J. Climatol., 35, 4843-4848.

Charney, J. G., and A. Eliassen, 1964: On the growth of the hurricane depression. $J$. Atmos. Sci., 21, 68-75.

Chavas, D. R., and K. A. Emanuel, 2010: A QuikSCAT climatology of tropical cyclone size, Geophys. Res. Lett., 37, L18816. 
Chavas, D. R., N. Lin, W. Dong, and Y. Lin, 2016: Observed tropical cyclone size revisited. J. Climate, 29, 2923-2939.

Chen, F., and J. Dudhia, 2001: Coupling an advanced land surface-hydrology model with the Penn State-NCAR MM5 modeling system. Part I: Model description and implementation. Mon. Wea. Rev., 129, 569-585.

Chen, H. and D. Zhang, 2013: On the rapid intensification of Hurricane Wilma (2005). Part II: Convective bursts and the upper-level warm core. J. Atmos. Sci., 70, 146-162.

Chen, H., and S. G. Gopalakrishnan, 2015: A study on the asymmetric rapid intensification of Hurricane Earl (2010) using the HWRF system. J. Atmos. Sci., 72, 531550 .

Chen, S. S., J. A. Knaff, and F. D. Marks Jr., 2006: Effects of vertical wind shear and storm motion on tropical cyclone rainfall asymmetries deduced from TRMM. Mon. Wea. Rev., 134, 3190-3208.

Cione, J. J., and E. W. Uhlhorn, 2003: Sea surface temperature variability in hurricanes: Implications with respect to intensity change. Mon. Wea. Rev., 131, 1783-1796.

Cione, J. J., E. A. Kalina, J. A. Zhang, and E. W. Uhlhorn, 2013: Observations of air-sea interaction and intensity change in hurricanes. Mon. Wea. Rev., 141, 2368-2382.

Corbosiero, K. L. and J. Molinari, 2003: The relationship between storm motion, vertical wind shear, and convective asymmetries in tropical cyclones. J. Atmos. Sci., 60, 366-376.

Craig, G. and S. Gray, 1996: CISK or WISHE as the mechanism for tropical cyclone intensification. J. Atmos. Sci., 53, 3528-3540.

D'Asaro, E., P. Black, L. Centurioni, Y. Chang, S. Chen, R. Foster, H. Graber, P. Harr, V. Hormann, R. Lien, I. Lin, T. Sanford, T. Tang, and C. Wu, 2014: Impact of typhoons on the ocean in the Pacific. Bull. Amer. Meteor. Soc., 95, 1405-1418.

Davis, C. and Coauthors, 2006: Advanced research WRF developments for hurricane prediction. Seventh WRF Users' Wokshop, Boulder, CO, NCAR, 3.1.

Davis, C., W. Wang, J. Dudhia, and R. Torn, 2010: Does increased horizontal resolution improve hurricane wind forecasts? Wea. Forecasting, 25, 1826-1841.

DeMaria, M., and J. Kaplan, 1994: A Statistical Hurricane Intensity Prediction Scheme (SHIPS) for the Atlantic basin. Wea. Forecasting, 9, 209-220. 
DeMaria, M., and J. Kaplan, 1999: An updated Statistical Hurricane Intensity Prediction Scheme (SHIPS) for the Atlantic and Eastern North Pacific basins. Wea. Forecasting, 14, 326-337.

DeMaria, M., J. Rhome, R. Pasch, and J. S. Clark, 2009: Technical summary of the National Hurricane Center track and intensity models, National Hurricane Center, Accessed 4 January 2017. [Available online at: http://www.nhc.noaa.gov/modelsummary.shtml]

Demuth, J., M. DeMaria, J. A. Knaff, and T. H. Vonder Haar, 2004: Validation of an Advanced Microwave Sounder Unit (AMSU) tropical cyclone intensity and size estimation algorithm. J. Appl. Meteor., 43, 282-296.

Demuth, J., M. DeMaria, and J. A. Knaff, 2006: Improvement of Advanced Microwave Sounding Unit tropical cyclone intensity and size estimation algorithms. J. Appl. Meteor. Climatol., 45, 1573-1581.

Draper, D. W., and D. G. Long, 2002: An assessment of SeaWinds on QuikSCAT wind retrieval. J. Geophys. Res., 107, 3212, doi:10.1029/2002JC001330.

Draper, D. W., and D. G. Long, 2004: Simultaneous wind and rain retrieval using SeaWinds data. IEEE Trans. Geosci. Remote Sens., 42, 1411-1423.

Dvorak, V. F., 1975: Tropical cyclone intensity analysis and forecasting from satellite imagery. Mon. Wea. Rev., 103, 420-430.

Dvorak, V. F., 1984: Tropical cyclone intensity analysis using satellite data. NOAA Tech. Rep. 11, 45 pp. [Available from NOAA/NESDIS, 5200 Auth Rd., Washington, DC 20333].

Elsberry, R. L., and R. A. Jeffries, 1996: Vertical wind shear influences on tropical cyclone formation and intensification during TCM-92 and TCM-93. Mon. Wea. Rev., $124,1374-1387$.

Elsberry, R., T. Lambert, and M. Boothe, 2007: Accuracy of Atlantic and Eastern North Pacific tropical cyclone intensity forecast guidance. Wea. Forecast., 22, 747-762.

Elsberry, R. L., and P. A. Harr, 2008: Tropical Cyclone Structure (TCS08) field experiment science basis, observational platforms, and strategy. Asia-Pac. J. Atmos. Sci., $44,1-23$.

Emanuel, K., 1986: An air-sea interaction theory for tropical cyclones. Part I: Steadystate maintenance. J. Atmos. Sci., 43, 585-605. 
Emanuel, K., 2005: Divine Wind: The History and Science of Hurricanes. Oxford University Press, $285 \mathrm{pp}$.

Figa-Saldaña, J., J. J. W. Wilson, E. Attema, R. Gelsthorpe, M. R. Drinkwater, and A. Stoffelen, 2002: The advanced scatterometer (ASCAT) on the meteorological operational (MetOp) platform: A follow on for European wind scatterometers. Can. J. Rem. Sens., $28,404-412$.

Fiorino, M., and R. L. Elsberry, 1989: Contributions to tropical cyclone motion by small, medium and large scales in the initial vortex. Mon. Wea. Rev., 117, 721-727.

Florida Commission on Hurricane Loss Projection (FCHLP), 2013: Florida Public Hurricane Loss Model 5.0. Accessed 22 November 2016. [Available online at https://www.sbafla.com/methodology/Portals/Methodology/ModelSubmissions/2013/201 30725_FIU_2011Standards_Submission.pdf].

Fontaine, B., S. Janicot, and V. Moron, 1995: Rainfall anomaly patterns and wind field signals over West Africa in August (1958-1989). J. Climate, 8, 1503-1510.

Foster, R. C., J. Zhang, and P. Black, 2016: Estimates of tropical cyclone surface wind inflow from satellite scatterometers, $32^{\text {nd }}$ AMS Conf. on Hurricanes and Tropical Meteorology, San Juan, Puerto Rico, 11C.5, Preprints.

Frank, W. M., and E. A. Ritchie, 1999: Effects of environmental flow upon tropical cyclone structure. Mon. Wea. Rev., 127, 2044-2061.

Frank, W. M., and E. A. Ritchie, 2001: Effects of vertical wind shear on the intensity and structure of numerically simulated hurricanes. Mon. Wea. Rev., 129, 2249-2269.

Franklin, J. L., S. J. Ford, S. E. Feuer, and F. D. Marks Jr., 1993: The kinematic structure of Hurricane Gloria (1985) determined from nested analyses of dropwindsonde and Doppler radar data. Mon. Wea. Rev., 121, 2433-2451.

Franklin, J. L., M. L. Black, and K. Valde, 2003: GPS dropwindsonde wind profiles in hurricanes and their operational implications. Wea. Forecasting, 18, 32-44.

Gall, R., J. Franklin, F. Marks, E. N. Rappaport, and F. Toepher, 2013: The Hurricane Forecast Improvement Project. Bull. Amer. Meteor. Soc., 94, 329-343.

Gamache, J., F. Marks, and F. Roux, 1995: Comparison of three airborne Doppler sampling techniques with airborne in situ wind observations in Hurricane Gustav (1990). J. Atmos. Oceanic Technol., 12, 171-181.

George, J. E., and W. M. Gray, 1976: Tropical cyclone motion and surrounding parameter relationships. J. Appl. Meteor., 15, 1252-1264. 
Gohil, B. S., R. Sikhakolli, and R. K. Gangwar, 2013: Development of geophysical model functions for Oceansat-2 scatterometer. IEEE Trans. Geosci. Remote Sens., 10, $377-380$.

Goodberlet, M. A., C. T. Swift, and J. C. Wilkerson, 1989: Remote sensing of ocean surface winds with the Special Sensor Microwave/Imager. J. Geophys. Res., 94, 14 54714555 .

Gonzalez III, I., A. Cotto, and H. E. Willoughby, 2015: Synthesis of vortex Rossby waves. Part II: Vortex motion and waves in the outer waveguide. J. Atmos. Sci., 72, 3958-3974.

Gopalakrishnan, S. G., F. Marks Jr., X. Zhang, J.-W. Bao, K.-S. Yeh, and R. Atlas, 2011: The experimental HWRF system: A study on the influence of horizontal resolution on the structure and intensity changes in tropical cyclones using and idealized framework. Mon. Wea. Rev., 139, 1762-1784.

Gopalakrishnan, S., S. Goldenberg, T. Quirino, X. Zhang, F. Marks, K. Yeh, R. Atlas, and V. Tallapragada, 2012: Toward improving high-resolution numerical hurricane forecasting: Influence of model horizontal grid resolution, initialization, and physics. Wea. Forecasting, 27, 647-666.

Gray, W. M., 1968: Global view of the origin of tropical disturbances and storms. Mon. Wea. Rev., 96, 669-700.

Gray, W. M., 1979: Hurricanes: Their formation, structure, and likely role in tropical circulation. Meteorology over the Tropical Oceans. D. B. Shaw (Ed.), Roy. Meteor. Soc., $155-218$.

Guimond, S. R., G. M. Heymsfield, and F. J. Turk, 2010: Multiscale observations of Hurricane Dennis (2005): The effects of hot towers on rapid intensification. J. Atmos. Sci., 67, 633-654.

Han, J. and H.-L. Pan, 2011: Revision of convection and vertical diffusion schemes in the NCEP Global Forecast System. Wea. Forecasting, 26, 520-533.

Hawkins, H. and D. Rubsam, 1968: Hurricane Hilda, 1964. Mon. Wea. Rev., 96, 428452.

Hence, D. A., and R. A. Houze Jr., 2011: Vertical structure of hurricane eyewalls as seem by the TRMM precipitation radar. J. Atmos. Sci., 68, 1637-1652.

Hendricks, E. A., M. T. Montgomery, and C. A. Davis, 2004: The role of "vertical" hot towers in the formation of Tropical Cyclone Diana (1984). J. Atmos. Sci., 61, 1209-1232. 
Hendricks, E. A., M. S. Peng, B. Fu, and T. Li, 2010: Quantifying environmental control on tropical cyclone intensity change. Mon. Wea. Rev., 138, 3243-3271.

Heymsfield, G. M., J. Simpson, J. Halverson, L. Tian, E. Ritchie, and J. Molinari, 2006: Structure of highly sheared Tropical Storm Chantal during CAMEX-4. J. Atmos. Sci., 63, $268-287$.

Hock, T. F. and J. L. Franklin, 1999: The NCAR GPS dropwindsonde. Bull. Amer. Met. Soc., 80, 407-420.

Hoffman, R. N., and S. Mark Leidner, 2005: An introduction to the near-real-time QuikSCAT data. Wea. Forecasting, 20, 476-493.

Holland, G. J., 1983a: Tropical cyclone motion: Environmental interaction plus a beta effect. J. Atmos. Sci., 40, 328-342.

Holland, G. J., 1983b: Angular momentum transports in tropical cyclones. Quart. J. Roy. Meteor. Soc., 109, 187-209.

Holland, G. J., 1984: Tropical cyclone motion: A comparison of theory and observation. J. Atmos. Sci., 41, 68-75.

Hong, S.-Y. and H.-L. Pan, 1996: Nonlocal boundary layer vertical diffusion in a medium-range forecast model. Mon. Wea. Rev., 124, 2322-2339.

Hopsch, S., C. Thorncroft, K. Hodges, and A. Aiyyer, 2007: West African storm tracks and their relationship to Atlantic tropical cyclones. J. Climate, 20, 2468-2483.

Hristova-Veleva, S. M., and Coauthors, 2013: Revealing the winds under the rain. Part I: Passive microwave rain retrievals using a new observation-based parameterization of subsatellite rain variability and intensity-algorithm description. J. Appl. Meteor. Climatol., 52, 2828-2848.

Iacono, M. J., J. S. Delamere, E. J. Mlawer, M. W. Shephard, S. A. Clough, and W. D. Collins, 2008: Radiative forcing by long-lived greenhouse gases: Calculations with the AER radiative transfer models, J. Geophys. Res., 113, D13103.

Janjic, Z., 2003: A nonhydrostatic model based on a new approach. Meteor. Atmos. Phys., 82, 271-285.

Jarvinen, B. R., C. J. Neumann, and M. A. S. Davis, 1984: A tropical cyclone data tape for the North Atlantic Basin, 1886-1983: Contents, limitations, and uses. NOAA Tech. Memo. 22, NWS/NHC, Miami, FL, 21 pp. 
Jiang, H., E. M. Ramirez, and D. J. Cecil, 2013: Convective and rainfall properties of tropical cyclone inner cores and rainbands from 11 Years of TRMM data. Mon. Wea. Rev., 141, 431-450.

Jones, R. W., 1977: Vortex motion in a tropical cyclone model. J. Atmos. Sci., 34, 15181527.

Jorgensen, D. P., 1984: Mesoscale and convective-scale characteristics of mature hurricanes. Part II: Inner core structure of Hurricane Allen (1980). J. Atmos. Sci., 41, $1287-1311$.

Jorgensen, D. P., E. J. Zipser, and M. A. LeMone, 1985: Vertical motions in intense hurricanes. J. Atmos. Sci., 42, 839-856.

Kaplan, J., and M. DeMaria, 2003: Large-scale characteristics of rapidly intensifying tropical cyclones in the North Atlantic basin. Wea. Forecasting, 18, 1093-1108.

Kaplan, J., M. DeMaria, and J. A. Knaff, 2010: A revised tropical cyclone rapid intensification index for the Atlantic and Eastern North Pacific basins. Wea. Forecasting, $25,220-241$.

Kaplan, J., P. Reasor, and M. DeMaria, 2014: Assessing the relationship between the large-scale and inner-core estimates of vertical shear. Preprints, 31st Conf. on Hurricanes and Tropical Meteorology, San Diego, CA, Amer. Meteor. Soc., 17C.4

Kepert, J., 2001: The dynamics of boundary layer jets within the tropical cyclone core. Part I: Linear theory. J. Atmos. Sci., 58, 2469-2484.

Kieper, M. E., and H. Jiang, 2012: Predicting tropical cyclone rapid intensification using the $37 \mathrm{GHz}$ ring pattern identified from passive microwave measurements, Geophys. Res. Lett., 39, L13804.

Klotz, B. W., and P. A. Kucera, 2012: Observations of coastally transitioning West African Mesoscale Convective Systems during NAMMA. Intl. J. of Geophys., 2012, 125 .

Klotz, B. W., and E. W. Uhlhorn, 2014: Improved stepped frequency microwave radiometer tropical cyclone surface winds in heavy precipitation. J. Atmos. Oceanic Technol., 31, 2392-2408.

Klotz, B. W., and H. Jiang, 2016: Global composites of surface wind speeds in tropical cyclones based on a 12 year scatterometer database. Geophys. Res. Lett., 43, 10,48010,488 . 
Klotz, B. W., and H. Jiang, 2017: Examination of surface wind asymmetries in tropical cyclones: Part I. General structure and wind shear impacts. In review, Mon. Wea. Rev.

Knabb, R. D., J. R. Rhome, and D. P. Brown, 2011: Tropical cyclone report: Hurricane Katrina. National Hurricane Center Tropical Cyclone Reports, http://www.nhc.noaa.gov/data/tcr/.

Knaff, J., D. Brown, J. Courtney, G. Gallina, and J. Beven, 2010: An evaluation of Dvorak technique-based tropical cyclone intensity estimates. Wea. Forecasting, 25, $1362-1379$.

Knaff, J. A., M. DeMaria, D. A. Molenar, C. R. Sampson, and M. G. Seybold, 2011: An automated, objective, multiple-satellite platform tropical cyclone surface wind analysis. J. Appl. Meteor. Climatol., 50, 2149-2166.

Kossin, J. P., and M. D. Eastin, 2001: Two distinct regimes in the kinematic and thermodynamic structure of the hurricane eye and eyewall. J. Atmos. Sci., 58, 1079-1090.

Kossin, J. P., J. A. Knaff, H. I. Berger, D. C. Herndon, T. A. Cram, C. S. Velden, R. J. Murnane, and J. D. Hawkins, 2007: Estimating hurricane wind structure in the absence of aircraft reconnaissance. Wea. Forecasting, 22, 89-101.

Kummerow, C., Y. Hong, W. S. Olson, S. Yang, R. F. Adler, J. McCollum, R. Ferraro, G. Petty, D-B. Shin, and T. T. Wilheit, 2001: The evolution of the Goddarad Profiling Algorithm (GPROF) for rainfall estimation from passive microwave sensors. J. Appl. Meteor., 40, 1801-1820.

Kuo, H.-L., 1969: Motions of vortices and circulating cylinder in shear flow with friction. J. Atmos. Sci., 26, 390-398.

Kurihara, Y. and R. Tuleya, 1974: Structure of a tropical cyclone developed in a threedimensional numerical simulation model. J. Atmos. Sci., 31, 893-919.

Kwon Y. C., and S. Lord, B. Lapenta, V. Tallapragada, Q. Liu and Z. Zhang, 2010: Sensitivity of air-sea exchange coefficients ( $\mathrm{Cd}$ and $\mathrm{Ch})$ on hurricane intensity. 29th Conference on Hurricanes and Tropical Meteorology, 13C.1, Tuscon, AZ.

La Seur, N. and H. Hawkins, 1963: An analysis of Hurricane Cleo (1958) based on data from research reconnaissance aircraft. Mon. Wea. Rev., 91, 694-709.

Landsea, C., and J. Franklin, 2013: Atlantic hurricane database uncertainty and presentation of a new database format. Mon. Wea. Rev., 141, 3576-3592. 
Lonfat, M., F. D. Marks Jr., S. S. Chen, 2004: Precipitation distribution in tropical cyclones using the Tropical Rainfall Measuring Mission (TRMM) microwave imager: A global perspective. Mon. Wea. Rev., 132, 1645-1660.

Lorenz, E. N., 1983: A history of prevailing ideas about the general circulation of the atmosphere. Bull. Amer. Meteor. Soc., 64, 730-734.

Mai, M., B. Zhang, X. Li, P. Hwang, and J. A. Zhang, 2016: Application of AMSR-E and AMSR2 low-frequency channel brightness temperature data for hurricane wind retrievals. IEEE Trans. Geosci. Remote Sens., 54, 4501-4512.

Malkus, J. S. and Riehl, H. (1960), On the dynamics and energy transformations in steady-state hurricanes. Tellus, 12, 1-20.

Marks, F. D., 1985: Evolution of the structure of precipitation in Hurricane Allen (1980). Mon. Wea. Rev., 113, 909-930.

Marks, F. D., R. A. Houze Jr., and J. F. Gamache, 1992: Dual-aircraft investigation of the inner core of Hurricane Norbert. Part I: Kinematic structure. J. Atmos. Sci., 49, 919-942.

Miller, B. I., 1958: Rainfall rates in Florida hurricanes. Mon. Wea. Rev., 86, 258-264.

Molinari, J., and S. Skubis, 1985: Evolution of the surface wind field in an intensifying tropical cyclone. J. Atmos. Sci., 42, 2865-2879.

Molinari, J., and D. Vollaro, 2010: Rapid intensification of a sheared tropical storm. Mon. Wea. Rev., 138, 3869-3885.

Montgomery, M. T., S. V. Nguyen, R. K. Smith, and J. Persing, 2009: Is WISHE essential for tropical cyclone intensification? Quart. J. Roy. Meteor. Soc., 135, 16971714.

Montgomery, M. T., J. A. Zhang, and R. K. Smith, 2014: An analysis of the observed low-level structure of rapidly intensifying and mature hurricane Earl (2010). Quart. J. Roy. Meteor. Soc., 140, 2132-2146.

Montgomery, M. T., J. Persing, and R. K. Smith, 2015: Putting to rest WISHE-ful misconceptions for tropical cyclone intensification. J. Adv. Model. Earth. Syst., 7, 92109.

Mueller, K. J., M. DeMaria, J. A. Knaff, J. P. Kossin, and T. H. Vonder Haar, 2006: Objective estimation of tropical cyclone wind structure from infrared satellite data. Wea. Forecasting, 21, 990-1005. 
Nesbitt, S. W., E. J. Zipser, and C. D. Kummerow, 2004: An examination of Version-5 rainfall estimates from TRMM microwave imager, precipitation radar, and rain gauges on global, regional, and storm scales. J. Appl. Meteor., 43, 1016-1036.

Nguyen, M. C., M. J. Reeder, N. E. Davidson, R. K. Smith, and M. T. Montgomery, 2011: Inner-core vacillation cycles during the intensification of Hurricane Katrina. Quart. J. Roy. Meteor. Soc., 137, 829-844.

Nguyen, S. V., R. K. Smith, and M. T. Montgomery, 2008: Tropical cyclone intensification and predictability in three dimensions. Quart. J. Roy. Meteor. Soc., 134, $563-582$.

Nolan, D. S., Y. Moon, and D. P. Stern, 2007: Tropical cyclone intensification from asymmetric convection: Energetics and efficiency. J. Atmos. Sci., 64, 3377-3405.

Nolan, D. S., and B. Klotz, 2016: Guidance on observational undersampling over the tropical cyclone lifecycle. Joint Hurricane Testbed year-1 report. [Available at: http://www.nhc.noaa.gov/jht/15-17reports/Nolan_yr1_annualrpt.pdf].

Olander, T. L., and C. S. Velden, 2007: The advanced Dvorak technique: Continued development of an objective scheme to estimate tropical cyclone intensity using geostationary infrared satellite imagery. Wea. Forecasting, 22, 287-298.

Ooyama, K. V., 1969: Numerical simulation of the life cycle of tropical cyclones. $J$. Atmos. Sci., 26, 3-40.

Pálmen, E., and H. Riehl, 1957: Budget of angular momentum and energy in tropical cyclones. J. of Meteorology, 14, 150-159.

Pielke, R., Jr., J. Gratz, C. Landsea, D. Collins, M. Saunders, and R. Musulin, 2008: Normalized hurricane damage in the United States: 1900-2005. Nat. Hazards Rev., 9, 29-42, 10.1061/(ASCE)1527-6988(2008)9:1(29).

Powell, M. D., 1980: Evaluations of diagnostic marine boundary-layer models applied to hurricanes, Mon. Wea. Rev., 108, 757-766.

Powell, M. D., 1982: The transition of the Hurricane Frederic boundary-layer wind field from the open Gulf of Mexico to landfall, Mon. Wea. Rev., 110, 1912-1932.

Powell, M. D., and S. H. Houston, 1996: Hurricane Andrew's landfall in South Florida. Part II: Surface wind fields and potential real-time applications. Wea. Forecasting, 11, 329-349.

Powell, M. D., S. H. Houston, L. R. Amat, and N. Morisseau-Leroy, 1998: The HRD real-time hurricane wind analysis system. J. Wind Eng. Ind. Aerodyn., 77-78, 53-64. 
Powell, M. D., P. J. Vickery, and T. Reinhold, 2003: Reduced drag coefficient for high wind speeds in tropical cyclones. Nature, 422, 279-283.

Pu, Z., X. Li, and E. J. Zipser, 2009: Diagnosis of the initial and forecast errors in numerical simulation of the rapid intensification of Hurricane Emily (2005). Wea. Forecasting., 24, 1236-1251.

Rappaport, E. N., and Coauthors, 2009: Advances and challenges at the National Hurricane Center. Wea. Forecasting, 24, 395-419.

Reasor, P. D., M. T. Montgomery, and L. D. Grasso, 2004: A new look at the problem of tropical cyclones in vertical shear flow: Vortex resiliency. J. Atmos. Sci., 61, 3-22.

Reasor, P. D., M. D. Eastin, and J. F. Gamache, 2009: Rapidly intensifying Hurricane Guillermo (1997). Part I: Low-wavenumber structure and evolution. Mon. Wea. Rev., $137,603-631$.

Reasor, P. D., and M. D. Eastin, 2012: Rapidly intensifying Hurricane Guillermo (1997), Part II: Resilience in shear. Mon. Wea. Rev., 140, 425-444.

Reasor, P. D., R. Rogers, and S. Lorsolo, 2013: Environmental flow impacts on tropical cyclone structure diagnosed from airborne Doppler radar composites. Mon. Wea. Rev., 141, 2949-2969.

Riehl H, and J. S. Malkus, 1958: On the heat balance in the equatorial trough zone. Geophysica, 6, 503-538.

Rodgers, E. B., S. W. Chung, and H. F. Pierce, 1994: A satellite observational and numerical study of precipitation characteristics in western North Atlantic tropical cyclones. J. Appl. Meteor., 33, 129-139.

Rogers, R., S. Aberson, M. Black, P. Black, J. Cione, P. Dodge, J. Gamache, J. Kaplan, M. Powell, J. Dunion, E. Uhlhorn, N. Shay, and N. Surgi, 2006: The Intensity Forecasting Experiment: A NOAA multiyear field program for improving tropical cyclone intensity forecasts. Bull. Amer. Meteor. Soc., 87, 1523-1537.

Rogers, R., and E. Uhlhorn, 2008: Observations of the structure and evolution of surface and flight-level wind asymmetries in Hurricane Rita (2005). Geophys. Res. Lett., 35, L22811.

Rogers, R., S. Lorsolo, P. Reasor, J. Gamache, and F. Marks, 2012: Multiscale analysis of tropical cyclone kinematic structure from airborne Doppler radar composites. Mon. Wea. Rev., 140, 77-99. 
Rogers, R, and Coauthors, 2013: NOAA's Hurricane Intensity Forecasting Experiment (IFEX): A Progress Report. Bull. Amer. Met. Soc., 94, 859-882.

Rogers, R., P. D. Reasor, and J. A. Zhang, 2015: Multiscale structure and evolution of Hurricane Earl (2010) during rapid intensification. Mon. Wea. Rev., 143, 536-562.

Rogers, R., J. A. Zhang, J. Zawislak, H. Jiang, G. R. Alvey III, E. J. Zipser, and S. N. Stevenson, 2016: Observations of the structure and evolution of Hurricane Edouard (2014) during intensity change. Part II: Kinematic structure and the distribution of deep convection. Mon. Wea. Rev., 144, 3355-3376.

Rossby, C. G., 1948: On the displacement and intensity change of atmospheric vortices. J. Mar. Res., 7, 175-187.

Rotunno, R. R., and K. A. Emanuel, 1987: An air-sea interaction theory for tropical cyclones. Part II: Evolutionary study using a nonhydrostatic axisymmetric numerical model. J. Atmos. Sci., 44, 542-561.

Sanger, N. T., M. T. Montgomery, R. K. Smith, and M. M. Bell, 2014: An observational study of tropical cyclone spinup in supertyphoon Jangmi (2008) from 24 to 27 September. Mon. Wea. Rev., 142, 3-28.

Schade, L. R. and K. A. Emanuel, 1999: The ocean's effect on the intensity of tropical cyclones: Results from a simple coupled atmosphere-ocean model. J. Atmos. Sci., 56, 642-651.

Schubert, W. H., and J. J. Hack, 1982: Inertial stability and tropical cyclone development. J. Atmos. Sci., 39, 1687-1697.

Shapiro, L. J., and H. E. Willoughby, 1982: The response of balanced hurricanes to local sources of heat and momentum. J. Atmos. Sci., 39, 378-394.

Shapiro, L. J., 1983: The asymmetric boundary layer flow under a translating hurricane. J. Atmos. Sci., 40, 1984-1998.

Shapiro, L. J., and M. T. Mongomery, 2015: Towards clarity on understanding tropical cyclone intensification. J. Atmos. Sci., 72, 3020-3031.

Simpson, R. H., and H. Riehl, 1981: The Hurricane and Its Impact. LSU Press, 398 pp.

Skamarock, W. C., and Coauthors, 2008: A description of the Advanced Research WRF version 3. NCAR Tech. Note NCAR/TN-475+STR, 113 pp.

Smith, R. K., 2006: Lectures on tropical cyclones, Ludwig Maximillian University of Munich, Accessed 8 January 2017. [Available at http://www.meteo.physik.unimuenchen.de/ roger/Lectures/Tropical_Cyclones/]. 
Smith, R. K., M. T. Montgomery, and S. V. Nguyen, 2009: Tropical cyclone spin-up revisited. Quart. J. Roy. Meteor. Soc., 135, 1321-1335.

Spencer, M. W., C. Wu, and D. G. Long, 1997: Tradeoffs in the design of a spaceborne scanning pencil beam scatterometer: Application to SeaWinds.. IEEE Trans. Geosci. Remote Sens., 35, 115-126.

Stewart, S., 2012: Tropical cyclone report: Hurricane Katia. National Hurricane Center Tropical Cyclone Reports, [Available at http://www.nhc.noaa.gov/data/tcr/].

Stiles, B. W., S. M. Hristova-Veleva, R. S. Dunbar, S. Chan, S. L. Durden, D. EstebanFernandez, E. Rodriguez, W. L. Poulsen, R. W. Gaston, and P. S. Callahan, 2010: Obtaining accurate ocean surface winds in hurricane conditions: A dual-frequency scatterometry approach. IEEE Trans. Geosci. Remote Sens., 48, 3101-3113.

Stiles, B. W., and R. S. Dunbar, 2010: A neural network technique for improving the accuracy of scatterometer winds in rainy conditions. IEEE Trans. Geosci. Remote Sens., 48, 3114-3122.

Stiles, B. W., R. E. Danielson, W. L. Poulsen, M. J. Brennan, S. Hristova-Veleva, T-P. Shen, and A. G. Fore, 2014: Optimized tropical cyclone winds from QuikSCAT: A neural network approach. IEEE Trans. Geosci. Remote Sens., 52, 7418-7434.

Susca-Lopata, G., J. Zawislak, E. J. Zipser, and R. F. Rogers, 2015: The role of observed environmental conditions and precipitation evolution in the rapid intensification of Hurricane Earl (2010). Mon. Wea. Rev., 143, 2207-2223.

Tallapragada, V., C. Kieu, Y. Kwon, S. Trahan, Q. Liu, Z. Zhang, and I. Kwon, 2014: Evaluation of storm structure from the operational HWRF during 2012 implementation. Mon. Wea. Rev., 142, 4308-4325.

Tallapragada, V., and Coauthors, 2015: Hurricane Weather Research and Forecasting (HWRF) model: 2015 scientific documentation, NCAR/TN-522+STR.

Tao, C., and H. Jiang, 2015: Distributions of shallow to very deep precipitation/convection in rapidly intensifying tropical cyclones. J. Climate, 28, 87918824.

Tao, C., H. Jiang, and J. Zawislak, 2017: The relative importance of stratiform and convective rainfall in rapidly intensifying tropical cyclones. Mon. Wea. Rev., 145, 795809. 
Thomsen, G. L., R. K. Smith, and M. T. Montgomery, 2015: Tropical cyclone flow asymmetries induced by a uniform flow revisited. J. Adv. Model. Earth. Syst., 5, 382405.

Thorncroft, C. D., and K. Hodges, 2001: African easterly wave variability and its relationship to Atlantic tropical cyclone activity. J. Climate, 14, 1166-1179.

Torn, R. D., and C. Snyder, 2010: Uncertainty of tropical cyclone best-track information. Wea. Forecasting, 25, 61-78.

Ueno, M., and M. Kunii, 2009: Some aspects of azimuthal wavenumber-one structure of typhoons represented in the JMA operational mesoscale analyses. J. Meteor. Soc. Japan, $87,615-633$.

Ueno, M., and K. Bessho, 2011: A statistical analysis of near-core surface wind asymmetries in typhoons obtained from QuikSCAT data. J. Meteor. Soc. Japan, 89, 225241.

Uhlhorn, E. W., and P. G. Black, 2003: Verification of remotely sensed sea surface winds in hurricanes. J. Atmos. Oceanic Technol., 20, 99-116.

Uhlhorn, E. W., P. G. Black, J. L. Franklin, M. Goodberlet, J. Carswell, and A. S. Goldstein, 2007: Hurricane surface wind measurements from an operational stepped frequency microwave radiometer. Mon. Wea. Rev., 135, 3070-3085.

Uhlhorn, E. W., and D. S. Nolan, 2012: Observational undersampling in tropical cyclones and implications for estimated intensity. Mon. Wea. Rev., 140, 825-840.

Uhlhorn, E. W., B. W. Klotz, T. Vukicevic, P. D. Reasor, and R. F. Rogers, 2014: Observed hurricane wind speed asymmetries and relationships to motion and environmental shear. Mon. Wea. Rev., 142, 1290-1311.

Velden, C. and Coauthors, 2006: The Dvorak tropical cyclone intensity estimation technique: A satellite-based method that has endured for over 30 years. Bull. Amer. Meteor. Soc., 87, 1195-1210.

Viltard, N., C. Burlaud, and C. D. Kummerow, 2006: Rain retrieval from TMI brightness temperature measurements using TRMM PR-based database. J. Appl. Meteor. And Clim., $45,455-456$.

Vukicevic, T., E. Uhlhorn, P. Reasor, and B. Klotz, 2014: A novel multiscale intensity metric for evaluation of tropical cyclone intensity forecasts. J. Atmos. Sci., 71, 12921304. 
Willoughby, H., J. Clos, and M. Shoreibah, 1982: Concentric eye walls, secondary wind maxima, and the evolution of the hurricane vortex. J. Atmos. Sci., 39, 395-411.

Willoughby, H. E., F. D. Marks Jr., and R. J. Feinberg, 1984: Stationary and moving convective bands in hurricanes. J. Atmos. Sci., 41, 3189-3211.

Willoughby, H. E., 1988: The dynamics of the tropical cyclone core. Aus. Met. Mag., 36, 183-191.

Willoughby, H. E., 1990: Gradient balance in tropical cyclones. J. Atmos. Sci., 47, 265274.

Wingo, M. T., and D. J. Cecil, 2010: Effects of vertical wind shear on tropical cyclone precipitation. Mon. Wea. Rev., 138, 645-662.

Zagrodnik, J. P. and H. Jiang, 2014: Rainfall, convection, and latent heating distributions in rapidly intensifying tropical cyclones. J. Atmos. Sci., 71, 2789-2809.

Zawislak, J., H. Jiang, G. R. Alvey III, E. J. Zipser, R. F. Rogers, J. A. Zhang, and S. N. Stevenson, 2016: Observations of the structure and evolution of Hurricane Edouard (2014) during intensity change. Part I: Relationship between the thermodynamic structure and precipitation. Mon. Wea. Rev., 44, 3333-3354.

Zhang, J. A., and E. W. Uhlhorn, 2012: Hurricane sea surface inflow angle and an observation-based parametric model. Mon. Wea. Rev., 140, 3587-3605.

Zhang, X., T. Quirino, K.-S. Yeh, S. Gopalakrishnan, F. Marks, S. Goldenberg, and S. Aberson, 2011: HWRFx: Improving hurricane forecasts with high-resolution modeling. Comput. Sci. Eng., 13, 13-21.

Zhu, P., Z. Zhu, S. Gopalakrishnan, R. Black, F. D. Marks, V. Tallapragada, J. A. Zhang, $\mathrm{X}$. Zhang, and C. Gao, 2015: Impact of subgrid-scale processes on eyewall replacement cycle of tropical cyclones in HWRF system. Geophys. Res. Lett., 42, 27-36. 
APPENDICES 


\section{Appendix 1 - Supplemental Wind Speed Composite Information for Chapter 2}

In order to provide additional background or perspective on information presented in the Chapter 2, several supplemental tables regarding basin statistics are discussed. Table A1 provides several relevant storm and basin characteristics (mean and standard deviation) for all cases and for individual basins. Additionally, characteristics for TC intensity are given. Well-known trends are conveyed in Table A1. For example, the RMW decreases with increasing intensity. North Atlantic cases tend to be positioned higher in latitude than the other basins, but Western Pacific TCs tend to have larger RMWs. The higher latitude of North Atlantic may contribute to the slightly larger RMWs, which would agree with results from Chan and Chan (2015).

Tables A2 and A3 provide statistical significance parameters for the motionrelative and shear-relative composites, respectively. Median quadrant-based p-values from a Kolmogorov-Smirnov test are given with bold values indicating statistical significance at $95 \%$. Additionally, the percentage of points with at least $90 \%$ significance is provided for each quadrant. In general, the basin-specific composites are all significant when evaluated against the global composite, with the exception of two quadrants of the NATL composite. TC intensity composites are also statistically significant when evaluated against the global composite. Shading of figures in the main text incorporate these p-values. 
Table A1. Provided are the mean and standard deviation of various parameters within the dataset. These parameters are presented for all cases and for the individual basins. Abbreviations in the table include: latitude (LAT), maximum scatterometer wind speed ( $\mathrm{U}_{\max }$ ), radius of maximum wind (RMW), tropical storms/depressions (TS), Category 1-2 hurricanes (HUR), and Category 3-5 hurricanes (MHUR).

\begin{tabular}{|c|c|c|c|c|c|c|c|c|c|c|c|}
\hline & Number & LAT $\left(^{\circ}\right)$ & $\begin{array}{l}\text { LAT } \\
\text { (Dev.) }\end{array}$ & $\begin{array}{c}U_{\max } \\
\left(m^{-1}\right)\end{array}$ & $\begin{array}{c}\mathrm{U}_{\max } \\
\text { (Dev.) }\end{array}$ & $\begin{array}{c}\text { RMW } \\
(\mathbf{k m})\end{array}$ & $\begin{array}{l}\text { RMW } \\
\text { (Dev.) }\end{array}$ & $\begin{array}{c}\text { MOTION } \\
\left(\mathrm{m} \mathrm{s}^{-1}\right)\end{array}$ & $\begin{array}{c}\text { MOTION } \\
\text { (Dev.) }\end{array}$ & $\begin{array}{c}\text { SHEAR } \\
\left(\mathrm{m} \mathrm{s}^{-1}\right)\end{array}$ & $\begin{array}{c}\text { SHEAR } \\
\text { (Dev.) }\end{array}$ \\
\hline \multicolumn{12}{|c|}{ All Cases } \\
\hline All & 2515 & 19.34 & 6.82 & 32.2 & 13.1 & 61.1 & 26.5 & 4.8 & 2.4 & 7 & 4.4 \\
\hline TS & 1392 & 18.81 & 7.12 & 23.7 & 6.4 & 68.2 & 28.4 & 4.7 & 2.4 & 7.7 & 4.8 \\
\hline HUR & 636 & 20.54 & 7.03 & 37.1 & 9.2 & 56.2 & 23.2 & 5 & 2.7 & 6.9 & 4 \\
\hline MHUR & 487 & 19.49 & 5.17 & 49.9 & 10.7 & 47.1 & 16.2 & 5 & 2.1 & 5.3 & 3.2 \\
\hline \multicolumn{12}{|c|}{ North Atlantic (NATL) } \\
\hline All & 540 & 25.02 & 8.45 & 32.4 & 13.7 & 65 & 27.8 & 5.7 & 2.7 & 8.1 & 4.7 \\
\hline TS & 305 & 24.92 & 8.95 & 23.7 & 6.5 & 73.9 & 29.8 & 5.5 & 2.8 & 8.9 & 5 \\
\hline HUR & 137 & 27.13 & 8.25 & 37.7 & 9.4 & 57.4 & 22.4 & 6.1 & 3.1 & 8 & 4.3 \\
\hline MHUR & 98 & 22.44 & 6.12 & 52.1 & 10.9 & 47.8 & 13.4 & 5.7 & 1.9 & 5.6 & 3.1 \\
\hline \multicolumn{12}{|c|}{ Eastern/Central Pacific } \\
\hline All & 602 & 17.03 & 3.83 & 26 & 9.3 & 58.3 & 26.1 & 4.4 & 1.9 & 6 & 4.1 \\
\hline TS & 431 & 17.3 & 4.21 & 21.6 & 4.6 & 65.1 & 27.1 & 4.3 & 2 & 6.5 & 4.5 \\
\hline HUR & 101 & 16.67 & 2.8 & 32.7 & 6.1 & 44.4 & 14.5 & 4.6 & 1.8 & 5.2 & 2.9 \\
\hline MHUR & 70 & 15.86 & 1.94 & 43.3 & 8.5 & 36.6 & 6.1 & 4.7 & 1.6 & 4.3 & 1.9 \\
\hline \multicolumn{12}{|c|}{ Western Pacific (WPAC) } \\
\hline All & 637 & 19.76 & 6.17 & 39.5 & 13.7 & 62.2 & 26.2 & 5 & 2.4 & 6.2 & 3.6 \\
\hline TS & 204 & 18.08 & 6.73 & 27.1 & 6.6 & 70.6 & 29.2 & 4.9 & 2.3 & 6.5 & 3.5 \\
\hline HUR & 226 & 20.44 & 6.42 & 38.9 & 10 & 63.8 & 26.5 & 5 & 2.6 & 6.7 & 4 \\
\hline MHUR & 207 & 20.66 & 4.87 & 52.3 & 10.7 & 52.3 & 18.7 & 5.2 & 2.2 & 5.3 & 3 \\
\hline \multicolumn{12}{|c|}{ Southern Hemisphere (SHEM) } \\
\hline All & 736 & -16.82 & 5.23 & 31 & 11.6 & 59.5 & 25.9 & 4.4 & 2.4 & 7.8 & 4.8 \\
\hline TS & 452 & -16.45 & 5.81 & 24.7 & 7 & 66.2 & 27.8 & 4.4 & 2.5 & 8.6 & 5 \\
\hline HUR & 172 & -17.68 & 4.35 & 36.7 & 8.4 & 52.1 & 19.4 & 4.2 & 2.4 & 7.2 & 4.1 \\
\hline MHUR & 112 & -17.02 & 3.6 & 47.5 & 9.9 & 43.4 & 13.4 & 4.4 & 2.1 & 5.8 & 4 \\
\hline
\end{tabular}


Table A2. Statistical significance parameters are provided for motion relative quadrants, where 'DMR' in the top labels refers to the down-motion-relative quadrant and so forth. Median p-values (PV) and percentage of points with at least $90 \%$ significance (PCT) are shown for each quadrant and then broken into several smaller sections, including: the region containing the INNER-core $(0.5-1.5 \mathrm{RMW})$, MID-range $(2 \leq \mathrm{r} / \mathrm{RMW} \leq 4)$, and OUTER-range ( $/ \mathrm{RMW}>4)$. Labels on the left refer to the specific composite, and they are evaluated against the global dataset. Values are provided for conditions where the motion vector was removed (MR) or not. P-values in bold print refer to significant (at 95\%) quadrants of the respective composite.

\begin{tabular}{|c|c|c|c|c|c|c|c|c|c|}
\hline & & DMR - PV & DMR - PCT & UMR - PV & UMR - PCT & $\mathbf{U M L}-\mathbf{P V}$ & UML - PCT & DML - PV & DML - PCT \\
\hline \multirow{8}{*}{ NATL } & FULL & 0.375 & 17.000 & 0.334 & 14.100 & 0.023 & 67.800 & 0.003 & 87.500 \\
\hline & INNER & 0.291 & 19.200 & 0.375 & 17.500 & 0.007 & 90.000 & 0.005 & 91.600 \\
\hline & MID & 0.358 & 13.400 & 0.221 & 18.400 & 0.010 & 68.800 & 0.001 & 90.800 \\
\hline & OUTER & 0.636 & 2.600 & 0.504 & 2.500 & 0.101 & 49.900 & 0.004 & 82.700 \\
\hline & FULL (MR) & 0.541 & 27.900 & 0.498 & 25.200 & 0.407 & 34.700 & 0.194 & 37.400 \\
\hline & INNER (MR) & 0.032 & 83.000 & 0.021 & 83.600 & 0.002 & 99.200 & 0.006 & 98.000 \\
\hline & MID (MR) & 0.668 & 1.300 & 0.654 & 2.200 & 0.503 & 15.000 & 0.247 & 22.000 \\
\hline & OUTER (MR) & 0.792 & 0.000 & 0.723 & 3.700 & 0.650 & 0.100 & 0.409 & 10.300 \\
\hline \multirow{8}{*}{ ЕРСР } & FULL & 0.000 & 100.000 & 0.000 & 100.000 & 0.000 & 94.700 & 0.000 & 99.000 \\
\hline & INNER & 0.000 & 100.000 & 0.000 & 100.000 & 0.000 & 100.000 & 0.000 & 100.000 \\
\hline & MID & 0.000 & 100.000 & 0.000 & 100.000 & 0.000 & 100.000 & 0.000 & 100.000 \\
\hline & OUTER & 0.000 & 100.000 & 0.000 & 100.000 & 0.000 & 87.000 & 0.000 & 97.600 \\
\hline & FULL (MR) & 0.000 & 100.000 & 0.000 & 100.000 & 0.000 & 99.800 & 0.000 & 99.700 \\
\hline & INNER (MR) & 0.000 & 100.000 & 0.000 & 100.000 & 0.000 & 100.000 & 0.000 & 100.000 \\
\hline & MID (MR) & 0.000 & 100.000 & 0.000 & 100.000 & 0.000 & 100.000 & 0.000 & 100.000 \\
\hline & OUTER (MR) & 0.000 & 100.000 & 0.000 & 99.900 & 0.000 & 99.600 & 0.000 & 99.200 \\
\hline
\end{tabular}




\begin{tabular}{|c|c|c|c|c|c|c|c|c|c|}
\hline \multirow{8}{*}{ WPAC } & FULL & 0.000 & 99.700 & 0.000 & 100.000 & 0.000 & 97.900 & 0.000 & 96.100 \\
\hline & INNER & 0.000 & 100.000 & 0.000 & 100.000 & 0.000 & 100.000 & 0.000 & 100.000 \\
\hline & MID & 0.000 & 100.000 & 0.000 & 100.000 & 0.000 & 100.000 & 0.000 & 100.000 \\
\hline & OUTER & 0.000 & 99.200 & 0.000 & 99.900 & 0.000 & 94.900 & 0.000 & 90.800 \\
\hline & FULL (MR) & 0.000 & 93.400 & 0.000 & 96.300 & 0.000 & 95.300 & 0.000 & 94.700 \\
\hline & INNER (MR) & 0.000 & 100.000 & 0.000 & 100.000 & 0.000 & 100.000 & 0.000 & 100.000 \\
\hline & MID (MR) & 0.000 & 100.000 & 0.000 & 100.000 & 0.000 & 100.000 & 0.000 & 100.000 \\
\hline & OUTER (MR) & 0.004 & 83.800 & 0.000 & 91.300 & 0.000 & 88.500 & 0.000 & 87.700 \\
\hline \multirow{8}{*}{ SHEM } & FULL & 0.193 & 30.300 & 0.252 & 34.400 & 0.297 & 22.800 & 0.018 & 75.800 \\
\hline & INNER & 0.168 & 28.000 & 0.104 & 48.900 & 0.268 & 15.400 & 0.124 & 42.700 \\
\hline & MID & 0.140 & 17.100 & 0.052 & 68.500 & 0.115 & 45.200 & 0.017 & 83.900 \\
\hline & OUTER & 0.108 & 47.800 & 0.581 & 4.200 & 0.601 & 14.900 & 0.005 & 93.400 \\
\hline & FULL (MR) & 0.149 & 33.500 & 0.240 & 17.700 & 0.592 & 4.900 & 0.498 & 7.100 \\
\hline & INNER (MR) & 0.240 & 10.700 & 0.155 & 29.900 & 0.391 & 5.500 & 0.385 & 4.000 \\
\hline & MID (MR) & 0.140 & 37.500 & 0.221 & 14.800 & 0.375 & 6.900 & 0.583 & 2.100 \\
\hline & OUTER (MR) & 0.108 & 45.700 & 0.328 & 9.900 & 0.890 & 0.000 & 0.506 & 12.300 \\
\hline \multirow{7}{*}{ TS } & FULL & 0.000 & 100.000 & 0.000 & 100.000 & 0.000 & 100.000 & 0.000 & 100.000 \\
\hline & INNER & 0.000 & 100.000 & 0.000 & 100.000 & 0.000 & 100.000 & 0.000 & 100.000 \\
\hline & MID & 0.000 & 100.000 & 0.000 & 100.000 & 0.000 & 100.000 & 0.000 & 100.000 \\
\hline & OUTER & 0.000 & 100.000 & 0.000 & 100.000 & 0.000 & 100.000 & 0.000 & 100.000 \\
\hline & FULL (MR) & 0.000 & 100.000 & 0.000 & 100.000 & 0.000 & 100.000 & 0.000 & 100.000 \\
\hline & INNER (MR) & 0.000 & 100.000 & 0.000 & 100.000 & 0.000 & 100.000 & 0.000 & 100.000 \\
\hline & MID (MR) & 0.000 & 100.000 & 0.000 & 100.000 & 0.000 & 100.000 & 0.000 & 100.000 \\
\hline
\end{tabular}




\begin{tabular}{|c|c|c|c|c|c|c|c|c|c|}
\hline & OUTER (MR) & 0.000 & 100.000 & 0.000 & 100.000 & 0.000 & 100.000 & 0.000 & 100.000 \\
\hline \multirow{8}{*}{ HUR } & FULL & 0.000 & 79.700 & 0.000 & 77.700 & 0.000 & 75.600 & 0.000 & 77.500 \\
\hline & INNER & 0.000 & 100.000 & 0.000 & 100.000 & 0.000 & 100.000 & 0.000 & 100.000 \\
\hline & MID & 0.000 & 100.000 & 0.000 & 100.000 & 0.000 & 100.000 & 0.000 & 100.000 \\
\hline & OUTER & 0.105 & 49.900 & 0.122 & 48.000 & 0.264 & 39.800 & 0.141 & 47.500 \\
\hline & FULL (MR) & 0.000 & 74.500 & 0.001 & 65.300 & 0.000 & 65.900 & 0.000 & 71.800 \\
\hline & INNER (MR) & 0.000 & 100.000 & 0.000 & 100.000 & 0.000 & 100.000 & 0.000 & 100.000 \\
\hline & MID (MR) & 0.000 & 100.000 & 0.000 & 98.400 & 0.000 & 99.500 & 0.000 & 100.000 \\
\hline & OUTER (MR) & 0.186 & 37.200 & 0.432 & 20.100 & 0.571 & 16.200 & 0.166 & 34.300 \\
\hline \multirow{7}{*}{ MHUR } & FULL & 0.000 & 100.000 & 0.000 & 100.000 & 0.000 & 100.000 & 0.000 & 100.000 \\
\hline & MID & 0.000 & 100.000 & 0.000 & 100.000 & 0.000 & 100.000 & 0.000 & 100.000 \\
\hline & OUTER & 0.000 & 100.000 & 0.000 & 100.000 & 0.000 & 100.000 & 0.000 & 100.000 \\
\hline & FULL (MR) & 0.000 & 100.000 & 0.000 & 100.000 & 0.000 & 100.000 & 0.000 & 100.000 \\
\hline & INNER (MR) & 0.000 & 100.000 & 0.000 & 100.000 & 0.000 & 100.000 & 0.000 & 100.000 \\
\hline & MID (MR) & 0.000 & 100.000 & 0.000 & 100.000 & 0.000 & 100.000 & 0.000 & 100.000 \\
\hline & OUTER (MR) & 0.000 & 100.000 & 0.000 & 100.000 & 0.000 & 100.000 & 0.000 & 100.000 \\
\hline
\end{tabular}


Table A3. Similar to Table A2, the statistical significance values are presented in a shear-relative reference frame. Naming conventions are the same as in Table A2.

\begin{tabular}{|c|c|c|c|c|c|c|c|c|c|}
\hline & & DSR - PV & DSR - PCT & USR - PV & USR - PCT & USL - PV & USL - PCT & DSL - PV & DSL - PCT \\
\hline \multirow{8}{*}{ NATL } & FULL & 0.515 & 9.600 & 0.039 & 62.700 & 0.018 & 72.200 & 0.460 & 12.700 \\
\hline & INNER & 0.525 & 5.100 & 0.006 & 91.600 & 0.001 & 99.300 & 0.281 & 24.000 \\
\hline & MID & 0.540 & 2.800 & 0.042 & 64.400 & 0.020 & 72.900 & 0.418 & 11.200 \\
\hline & OUTER & 0.560 & 0.200 & 0.153 & 43.700 & 0.089 & 52.400 & 0.641 & 0.200 \\
\hline & FULL (MR) & 0.345 & 29.000 & 0.356 & 30.200 & 0.486 & 31.600 & 0.491 & 22.600 \\
\hline & INNER (MR) & 0.035 & 80.800 & 0.001 & 100.000 & 0.008 & 99.200 & 0.048 & 77.100 \\
\hline & MID (MR) & 0.524 & 4.400 & 0.516 & 8.200 & 0.644 & 2.500 & 0.614 & 2.400 \\
\hline & OUTER (MR) & 0.608 & 1.700 & 0.484 & 1.300 & 0.673 & 0.000 & 0.648 & 0.100 \\
\hline \multirow{8}{*}{ EPCP } & FULL & 0.000 & 100.000 & 0.000 & 94.000 & 0.000 & 99.000 & 0.000 & 100.000 \\
\hline & INNER & 0.000 & 100.000 & 0.000 & 100.000 & 0.000 & 100.000 & 0.000 & 100.000 \\
\hline & MID & 0.000 & 100.000 & 0.000 & 1.000 & 0.000 & 100.000 & 0.000 & 100.000 \\
\hline & OUTER & 0.000 & 100.000 & 0.000 & 86.700 & 0.000 & 97.600 & 0.000 & 100.000 \\
\hline & FULL (MR) & 0.000 & 100.000 & 0.000 & 92.300 & 0.000 & 99.100 & 0.000 & 100.000 \\
\hline & INNER (MR) & 0.000 & 100.000 & 0.000 & 100.000 & 0.000 & 100.000 & 0.000 & 100.000 \\
\hline & MID (MR) & 0.000 & 100.000 & 0.000 & 100.000 & 0.000 & 100.000 & 0.000 & 100.000 \\
\hline & OUTER (MR) & 0.000 & 99.800 & 0.006 & 83.300 & 0.000 & 97.700 & 0.000 & 100.000 \\
\hline \multirow{4}{*}{ WPAC } & FULL & 0.000 & 97.500 & 0.000 & 96.800 & 0.000 & 99.800 & 0.000 & 99.500 \\
\hline & INNER & 0.000 & 100.000 & 0.000 & 100.000 & 0.000 & 100.000 & 0.000 & 100.000 \\
\hline & MID & 0.000 & 100.000 & 0.000 & 100.000 & 0.000 & 100.000 & 0.000 & 100.000 \\
\hline & OUTER & 0.000 & 93.900 & 0.000 & 92.600 & 0.000 & 99.500 & 0.000 & 98.800 \\
\hline
\end{tabular}




\begin{tabular}{|c|c|c|c|c|c|c|c|c|c|}
\hline & FULL (MR) & 0.000 & 92.900 & 0.000 & 90.700 & 0.000 & 94.600 & 0.000 & 97.300 \\
\hline & INNER (MR) & 0.000 & 100.000 & 0.000 & 100.000 & 0.000 & 100.000 & 0.000 & 100.000 \\
\hline & MID (MR) & 0.000 & 100.000 & 0.000 & 100.000 & 0.000 & 100.000 & 0.000 & 100.000 \\
\hline & OUTER (MR) & 0.002 & 82.600 & 0.016 & 78.400 & 0.000 & 86.600 & 0.000 & 93.700 \\
\hline \multirow{8}{*}{ SHEM } & FULL & 0.162 & 35.000 & 0.258 & 12.200 & 0.289 & 14.400 & 0.203 & 26.000 \\
\hline & INNER & 0.316 & 7.300 & 0.230 & 16.000 & 0.188 & 23.700 & 0.270 & 12.900 \\
\hline & MID & 0.202 & 22.700 & 0.191 & 20.800 & 0.180 & 25.100 & 0.267 & 13.100 \\
\hline & OUTER & 0.074 & 65.100 & 0.349 & 5.000 & 0.493 & 4.600 & 0.117 & 44.400 \\
\hline & FULL (MR) & 0.402 & 4.600 & 0.338 & 15.000 & 0.409 & 10.700 & 0.541 & 2.100 \\
\hline & INNER (MR) & 0.284 & 9.600 & 0.157 & 28.800 & 0.306 & 15.200 & 0.520 & 3.100 \\
\hline & MID (MR) & 0.475 & 2.500 & 0.187 & 23.900 & 0.238 & 17.700 & 0.731 & 1.260 \\
\hline & OUTER (MR) & 0.429 & 1.800 & 0.780 & 0.400 & 0.651 & 1.000 & 0.453 & 1.250 \\
\hline \multirow{8}{*}{ TS } & FULL & 0.000 & 100.000 & 0.000 & 100.000 & 0.000 & 100.000 & 0.000 & 100.000 \\
\hline & INNER & 0.000 & 100.000 & 0.000 & 100.000 & 0.000 & 100.000 & 0.000 & 100.000 \\
\hline & MID & 0.000 & 100.000 & 0.000 & 100.000 & 0.000 & 100.000 & 0.000 & 100.000 \\
\hline & OUTER & 0.000 & 100.000 & 0.000 & 100.000 & 0.000 & 100.000 & 0.000 & 100.000 \\
\hline & FULL (MR) & 0.000 & 100.000 & 0.000 & 100.000 & 0.000 & 100.000 & 0.000 & 100.000 \\
\hline & INNER (MR) & 0.000 & 100.000 & 0.000 & 100.000 & 0.000 & 100.000 & 0.000 & 100.000 \\
\hline & MID (MR) & 0.000 & 100.000 & 0.000 & 100.000 & $\mathbf{0 . 0 0 0}$ & 100.000 & 0.000 & 100.000 \\
\hline & OUTER (MR) & 0.000 & 100.000 & 0.000 & 100.000 & 0.000 & 100.000 & 0.000 & 100.000 \\
\hline \multirow{2}{*}{ HUR } & FULL & 0.000 & 76.000 & 0.000 & 64.700 & 0.000 & 77.800 & 0.000 & 76.600 \\
\hline & INNER & 0.000 & 100.000 & 0.000 & 100.000 & 0.000 & 100.000 & 0.000 & 100.000 \\
\hline
\end{tabular}




\begin{tabular}{|c|c|c|c|c|c|c|c|c|c|}
\hline & MID & 0.000 & 100.000 & 0.000 & 100.000 & 0.000 & 100.000 & 0.000 & 100.000 \\
\hline & OUTER & 0.197 & 40.900 & 0.397 & 17.500 & 0.184 & 45.200 & 0.180 & 45.500 \\
\hline & FULL (MR) & 0.001 & 76.200 & 0.002 & 60.400 & 0.000 & 77.300 & 0.000 & 71.300 \\
\hline & INNER (MR) & 0.000 & 100.000 & 0.000 & 100.000 & 0.000 & 100.000 & 0.000 & 100.000 \\
\hline & MID (MR) & 0.000 & 99.000 & 0.001 & 97.100 & 0.000 & 100.000 & 0.000 & 100.000 \\
\hline & OUTER (MR) & 0.118 & 42.000 & 0.332 & 9.700 & 0.144 & 44.000 & 0.336 & 33.000 \\
\hline \multirow{8}{*}{ MHUR } & FULL & 0.000 & 100.000 & 0.000 & 100.000 & 0.000 & 100.000 & 0.000 & 100.000 \\
\hline & INNER & 0.000 & 100.000 & 0.000 & 100.000 & 0.000 & 100.000 & 0.000 & 100.000 \\
\hline & MID & 0.000 & 100.000 & 0.000 & 100.000 & 0.000 & 100.000 & 0.000 & 100.000 \\
\hline & OUTER & 0.000 & 100.000 & 0.000 & 100.000 & 0.000 & 100.000 & 0.000 & 100.000 \\
\hline & FULL (MR) & 0.000 & 100.000 & 0.000 & 100.000 & 0.000 & 100.000 & 0.000 & 100.000 \\
\hline & INNER (MR) & 0.000 & 100.000 & 0.000 & 100.000 & 0.000 & 100.000 & 0.000 & 100.000 \\
\hline & MID (MR) & 0.000 & 100.000 & 0.000 & 100.000 & 0.000 & 100.000 & 0.000 & 100.000 \\
\hline & OUTER (MR) & 0.000 & 100.000 & 0.000 & 100.000 & 0.000 & 100.000 & 0.000 & 100.000 \\
\hline
\end{tabular}


VITA

BRADLEY KLOTZ

Born, Houston, TX, USA

2002-2006

B.S., Meteorology

Texas A\&M University

College Station, TX, USA

2006-2008

M.S., Atmospheric Science

University of North Dakota

Grand Forks, ND, USA

2009 - Present

Senior Research Associate

Cooperative Institute for Marine and Atmospheric

Studies, University of Miami

NOAA, Hurricane Research Division

Miami, FL, USA

$2013-2015$

Doctoral Graduate Student

Florida International University

Miami, FL, USA

2015-Present

Doctoral Candidate

Florida International University

Miami, FL, USA

\section{SELECTED PUBLICATIONS AND PRESENTATIONS}

Klotz, B. W., and H. Jiang, 2017: Examination of surface wind asymmetries in tropical cyclones: Part II. Intensity change, Monthly Weather Review, in review.

Klotz, B. W., and H. Jiang, 2017: Examination of surface wind asymmetries in tropical cyclones: Part I. General structure and wind shear impacts, Monthly Weather Review, in review.

Klotz, B. W., and H. Jiang, 2016: Global composites of surface wind speeds in tropical cyclones based on a 12 year scatterometer database. Geophysical Research Letters, 43, $10,480-10,488$.

Klotz, B. W., and E. W. Uhlhorn, 2014: Improved Stepped Frequency Microwave Radiometer Tropical Cyclone Surface Winds in Heavy Precipitation, Journal of Atmospheric and Oceanic Technology, 31, 2392-2408.

Klotz, B. W. and P. Kucera, 2012: Observations of coastally transitioning West African 
Mesoscale Convective Systems during NAMMA, International Journal of Geophysics, vol. 2012, Article ID 438706, 25 pages, doi:10.1155/2012/438706.

Klotz, B. W., and H. Jiang, 2017: Tropical cyclone surface wind asymmetry in response to wind shear and intensity change. FIU Earth and Environment Graduate Student Symposium, Miami, FL, 24 February, 2017.

Klotz, B. W., and H. Jiang, 2016: Using a scatterometer-based, low-wavenumber analysis tool to evaluate surface wind asymmetries in tropical cyclones. $32^{\text {nd }}$ AMS Conference on Hurricanes and Tropical Meteorology, San Juan, Puerto Rico, 20 April, 2016.

Klotz, B. W., E. W. Uhlhorn, A. Goldstein, and I. PopStefanija, Performance of the Revised SFMR Algorithm: 2015 Season in Review, 94 (poster), AMS 32nd Conference on Hurricanes and Tropical Meteorology, San Juan, PR. April 19, 2016.

Klotz, B. W., D. S. Nolan, and E. W. Uhlhorn, Further Studies of Observational Undersampling in Flight-level and SFMR Observations, 150 (poster), AMS 32nd Conference on Hurricanes and Tropical Meteorology, San Juan, PR. April 21, 2016.

Klotz, B. W., E. Uhlhorn, R. Black, and S. Lorsolo: Further improvement of SFMR Surface Wind Speeds in Heavy Precipitation. $31^{\text {st }}$ AMS Conference on Hurricanes and Tropical Meteorology, San Diego, CA, 3 April, 2014.

AWARDS AND RECOGNITION

FIU Worlds Ahead Graduate, May 2017 